\title{
Role of Nitric Oxide and Reactive Oxygen Species in Apoptotic, Inflammatory and Migratory Signaling Pathways in Cancer
}

\author{
Siera Jo Talbott \\ West Virginia University
}

Follow this and additional works at: https://researchrepository.wvu.edu/etd

\section{Recommended Citation}

Talbott, Siera Jo, "Role of Nitric Oxide and Reactive Oxygen Species in Apoptotic, Inflammatory and Migratory Signaling Pathways in Cancer" (2011). Graduate Theses, Dissertations, and Problem Reports. 4802.

https://researchrepository.wvu.edu/etd/4802

This Dissertation is protected by copyright and/or related rights. It has been brought to you by the The Research Repository @ WVU with permission from the rights-holder(s). You are free to use this Dissertation in any way that is permitted by the copyright and related rights legislation that applies to your use. For other uses you must obtain permission from the rights-holder(s) directly, unless additional rights are indicated by a Creative Commons license in the record and/ or on the work itself. This Dissertation has been accepted for inclusion in WVU Graduate Theses, Dissertations, and Problem Reports collection by an authorized administrator of The Research Repository @ WVU.

For more information, please contact researchrepository@mail.wvu.edu. 
Role of Nitric Oxide and Reactive Oxygen Species in Apoptotic, Inflammatory and Migratory Signaling Pathways in Cancer

Siera Jo Talbott

Dissertation Submitted to the School of Medicine at West Virginia University

In Partial Fulfillment of the Requirements for the Degree of

Doctor of Philosophy in Cancer Cell Biology

\author{
Laura Gibson, Ph.D., Chair \\ Scott Weed, Ph.D. \\ Linda Vona-Davis, Ph.D. \\ Patrick Callery, Ph.D. \\ Karen Martin, Ph.D. \\ Yon Rojanasakul, Ph.D., mentor \\ Cancer Cell Biology Program \\ Morgantown, West Virginia \\ 2011
}

Keywords: FLIP, RIP, reactive oxygen species, nitric oxide, S-nitrosylation, caveolin-1, NF-kB 


\title{
ABSTRACT \\ Role of Nitric Oxide and Reactive Oxygen Species in Apoptotic, Inflammatory and Migratory Signaling Pathways in Cancer
}

\author{
Siera Jo Talbott
}

Cancer represents over 200 different diseases of different cell and tissue type origins. The heterogeneity of tumors presents challenges in finding effective treatments for the different diseases. Therefore, understanding the molecular events that promote tumorgenesis can assist in the development of better targeted treatments and therapies. Reduction/oxidation (redox) biology has become increasingly important as high levels of nitric oxide (NO) and reactive oxygen species (ROS) are reported in both the development of cancer and in response to treatment. In addition to their known potential biotoxic effects on DNA, proteins and lipids, NO and ROS are recognized as secondary messengers within the cell that mediate specific signaling events. The microenvironment can additionally be a source of NO and ROS that promote chronic inflammation and tumorigenesis. Additionally, tumor cells are reported to function with higher levels of oxidative stress indicating the signaling effects of $\mathrm{NO}$ and ROS are of even higher importance. We are primarily interested in how $\mathrm{NO}$ and ROS can mediate signaling changes and participate in the advancement of cancer.

Death receptors $(D R)$ are transmembrane receptors that mediate cytokine signaling via cognate ligands to determine the fate of the cell. DR signaling can lead to cell survival and proinflammatory responses or apoptotic cell death. DR ligands such as Tumor Necrosis Factor alpha (TNF- $\alpha$ ) and Fas Ligand (FasL) are abundant during infection and pathogenesis and are an important factor in both tumorigenesis and cancer treatment. DR-mediated apoptotic resistance is a major problem in effective cancer treatments and is usually marked by cancer cells upregulating intracellular antiapoptotic proteins.

In the first study, we examine the effects of S-nitrosylation on antiapoptotic protein FLICE Inhibitory Protein (FLIP) which is highly expressed in chemotherapeutic-resistant tumors. We performed apoptosis assays using fluorescence microscopy and report that FLIP antiapoptotic activity is dependent on S-nitrosylation at cysteines 254 and 259. By using dual-luciferase reporter assays we demonstrate that S-nitrosylated FLIP mediates potent proinflammatory transcription factor Nuclear Factor kappa B (NF-кB). Receptor Interacting Protein 1 (RIP1) and Tumor Necrosis Factor Receptor-associated Factor 2 (TRAF2) are two essential adaptor proteins upstream of NF-kB activation. Upon coimmunoprecipitation and confocal microscopy we were able to determine FLIP interaction with RIP1 and not TRAF2 was dependent on Snitrosylation status and most likely mediates NF- $\mathrm{kB}$ activity. Additionally through 
standard Western blotting, we show that FLIP processing and cleavage is dependent on S-nitrosylation status. Taken together, our results indicate the Snitrosylation posttranslational modification of FLIP is a potent mediator of cell fate. It can allow cancer cells apoptotic resistance while signaling for inflammation and proliferation. This deleterious combination could promote tumor survival selectivity in chemotherapeutic resistant cancers.

Cellular migration is a necessary component of invasion and metastasis in cancer. Caveolin-1 (Cav-1) is the essential constituent of cholesterol enriched lipid raft invaginations known as caveolae. Cav-1 is polarized in migratory cells indicating a role in motility and it has been reported to affect migration. Cav-1 can act as a scaffolding protein and has been reported as both a tumor promoter and suppressor determined mostly by protein binding partners.

In the second study, we examine the effects of different ROS species on migration in non-small cell lung cancer (NSCLC) cells. By using wound healing and Transwell assays we determined $\mathrm{O}_{2}{ }^{-}$and $\mathrm{H}_{2} \mathrm{O}_{2}$ have an inhibitory effect whereas $\mathrm{OH}$. promotes NSCLC migration and invasion. Through Cav-1 knockdown and overexpression experiments we were able to determine the ROS-mediated migration and invasion occurs in a Cav-1-dependent manner. Furthermore, we show the inhibitory roles of $\mathrm{O}_{2}{ }^{-}$and $\mathrm{H}_{2} \mathrm{O}_{2}$ on Cav-1 expression are mediated through the ubiquitin-proteosome pathway. Our laboratory and others have shown Cav-1-mediated migration occurs through PI3K/Akt signaling. Therefore, we confirmed the ROS-mediated Cav-1-dependent migration affected Akt phosphorylation consistent with the known migratory process. This is the first report linking $\mathrm{OH}$. and Cav-1-dependent migration and invasion.

In summary, these studies examine the effects of NO and ROS on important signaling pathways in cancer progression. Specifically we show how $\mathrm{NO}$ and ROS can selectively target apoptotic, proliferative and migratory processes which could dramatically enhance tumorigenesis in vivo. With an abundance of NO and ROS reported in the tumor and the supporting microenvironment, this research further expands on the limited knowledge of implications of redox biology in cancer signaling. 


\section{ACKNOWLEDGEMENTS}

The body of this work could not have been completed without the help of many individuals. I would first like to thank my mentor, Dr. Yon Rojanasakul, for his encouragement to aim high in my endeavors and seek success from all avenues of life. The freedom that he allowed me during my time in his laboratory led me to carve out my own scientific ideas and find the best way to test them. He has been an incredibly supportive mentor. I would also like to thank my committee members Dr. Scott Weed and Dr. Laura Gibson for their sincere guidance and mentorship as well as their generosity in letting me "borrow" the occasional reagent. I would also like to thank committee member Dr. Karen Martin for always being approachable, helping me find paths through research brick walls and for letting me ask seemingly dumb questions without letting me know it. I would additionally like to thank my committee member Dr. Patrick Callery for making me think outside the constraints of classic molecular biology and challenging my chemistry database. And lastly, my committee member Dr. Linda Vona-Davis, who I truly believe is my fairy godmother. She brought me to the Health Science Center Biomedical Graduate Studies Program and henceforth, my entire life changed.

I would also like to thank all the current and past members of the laboratory for providing a supportive and entertaining environment. I still believe that our lab luncheons and parties have had the best food in Morgantown. I especially enjoyed working with Dr. Nicole Bryan, who was always willing to debate. I also would like to specifically thank Sudjit Luanpitpong for her teamwork and incredible work ethic. Additionally, I thank our technician, Yongju Lu for her willingness to help and her amazing smile. I especially must thank Dr. Christian Stehlik, whom I had the pleasure to work under for 2 years. His scientific ingenuity and altruism have been priceless in my project and have made me a better scientist. 
During my graduate career I have been a part of two fantastic departments; the Cancer Cell Biology Program and the Department of Basic Pharmaceutical Sciences. I have been fortunate to be part of a graduate school program that fosters interaction and communication between students. I am most grateful for the lifelong friendships that I have fostered from WVU. Dr. Laura Kelley, Dr. Janna Jackson, Dr. Tricia Lewis, Dr. Heather O'Leary and Dr. Kelly Bailey have all brought out the best in me and have provided a steadfast professional and personal support system. They have lent me their strength on bike rides, their supplies in lab, their acumen during brain freezes, their wine when I had none and their counsel always. I consider myself incredibly fortunate to have met such lovely women in science and made such great friends in life.

Finally, I would like to thank my family for their patience during my graduate career. My father has been my inspiration and my biggest advocate. His life theories have taught me to persevere and pursue avenues that are seemingly out of reach. I thank my mother for demonstrating an unshakeable work ethic and my sister, Laura for teaching me to run and believing in my greatness. I must thank my daughter for her endless distraction and constant source of amusement. And I truly thank her for being an easy baby. Lastly I am entirely indebted to my husband, Dr. Brian Talbott, whom I met the day I started the graduate program. He has been a part of every step along the way. His companionship, love, support and humor have been life's greatest gift (of course, his ability to whooo at my data has been appreciated too). He persuaded me to see that life from a bicycle is a better way to view the world and a great way to spend your time. Words cannot express the gratitude I have for him. I could not have completed this project without his support. 


\section{Table of Contents}

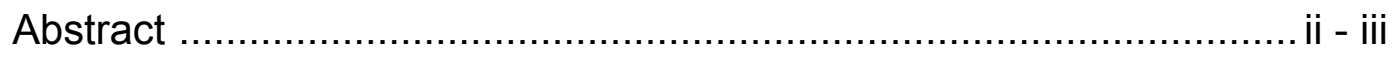

Acknowledgements .................................................................

Table of Contents.......................................................................... vi vii

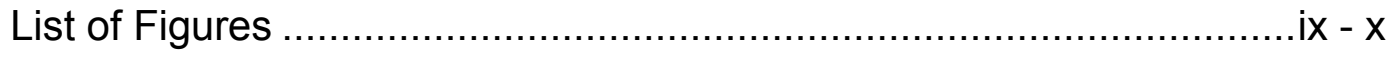

Literature Review ................................................................

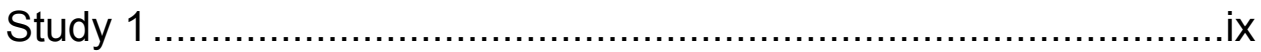

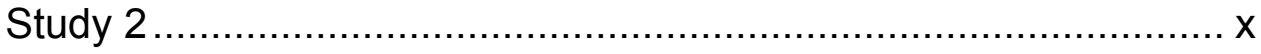

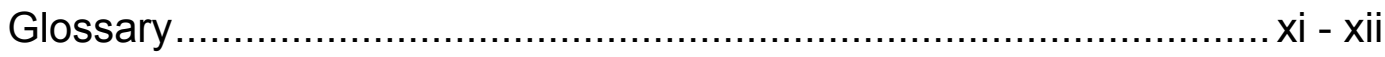

Chapter 1. Literature Review ...................................................... 1 - 56

Cancer as a NO and ROS signaling disease ............................. 1

Reactive oxygen species ................................................ 2

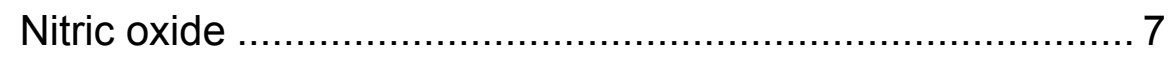

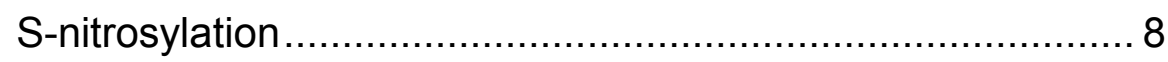

Reactive nitrogen species ……................................... 10

Death receptor signaling - apoptotic........................................... 11

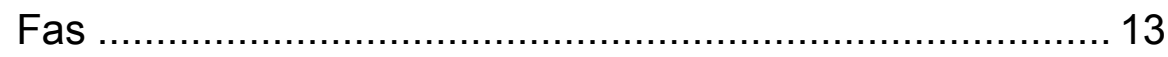

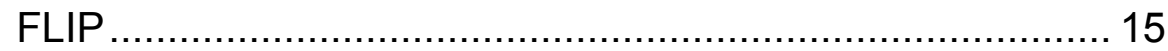

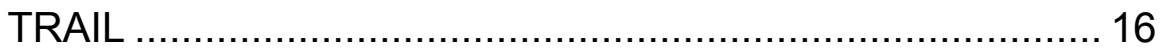

Death receptor signaling - non-apoptotic .................................. 17

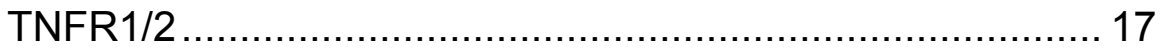

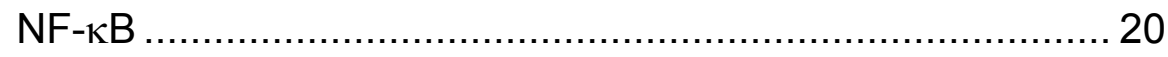

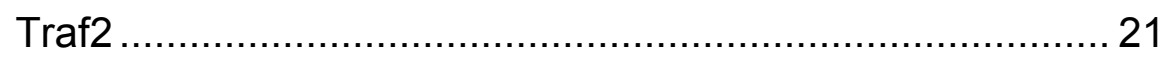

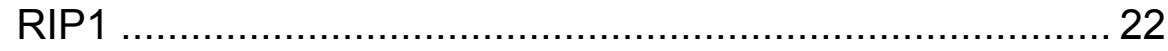

Divergent death receptor signaling ......................................... 23 
Death receptor signaling in disease .......................................... 24

Cell migration ................................................................ 30

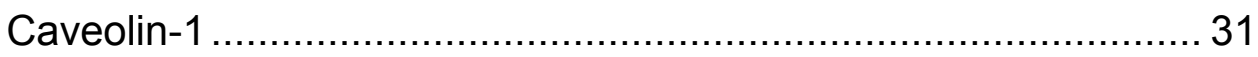

Impact and implications of studies ........................................ 35

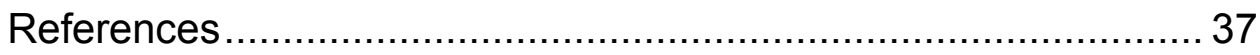

Chapter 2. Study 1: S-Nitrosylation of FLICE Inhibitory Protein Determines Its Interaction with RIP-1 and Activation of NF-kB .........57 - 95

Abstract

Chapter 3. Study 2: Regulation of Lung Cancer Cell Migration and Invasion by Reactive Oxygen Species and Caveolin-1 f.................. 96 - 140

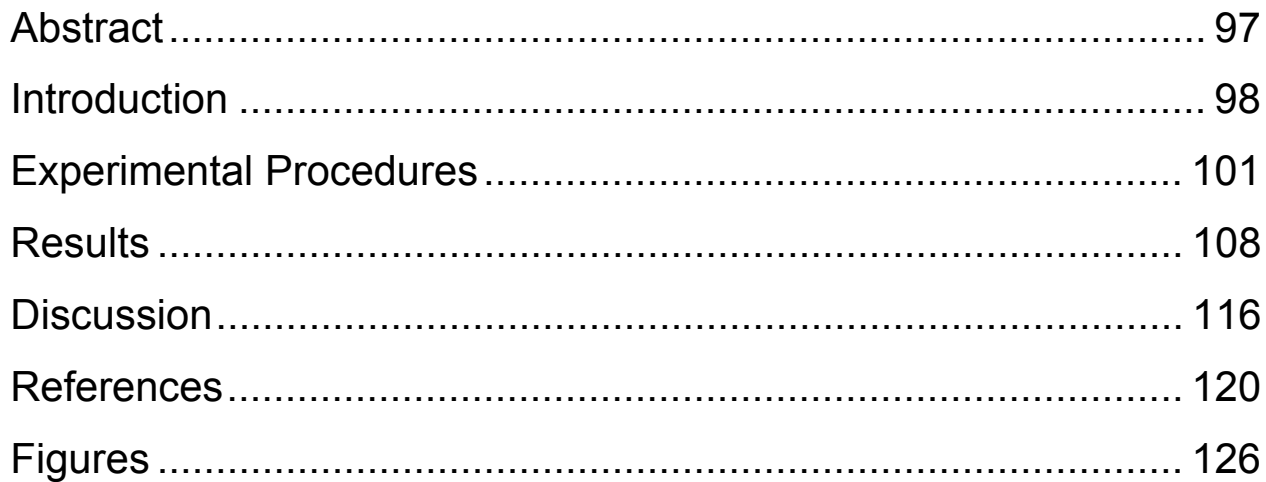

Chapter 4. General Discussion ................................................ 141 - 150

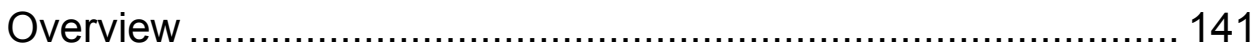


S-nitrosylation of FLIP moderates its function

Varying effects of ROS species on Cav-1-dependent migration in NSCLC cells.

Future directions and recommendations 144

References

Appendix $151-155$

Study 3. Nitric oxide regulates lung carcinoma cell anoikis through inhibition of ubiquitin-proteosomal degradation of Caveolin-1 152

Study 4. A Shope fibroma virus PYRIN-only protein modulates the host immune response 160 Study 5. Cellular pyrin domain-only protein 2 is a candidate regulator of inflammasome activation 170 Curriculum Vitae. 179 


\section{List of Figures}

\section{Chapter 1. Literature Review}

1. Flow chart demonstrating multiple stimuli leads to the production of ROS and RNS which mediate many redox-dependent signaling pathways.

2. Schematic of how FLIP concentration alters DISC-dependent apoptosis; and inflammation and proliferation signaling through other complexes.

3. Schematic of TNFR1/2 signaling results in two complexes

\section{Chapter 2. Study 1}

1. Schematic of FLIP constructs used in experimentation including a double cysteine mutant which is unable to be S-nitrosylated at residues 254 and 259 and experimental assessment of total S-nitrosylation by immunoprecipitation and subsequent fluorescent measurement or Western blotting.

2. Dual luciferase experiments demonstrating FLIP mediates NF-kB activation which is dependent on both the two $\mathrm{N}$-terminal domains and S-nitrosylation status. Protein expression verified by Western blot.

3. Apoptotic assays by Hoechst 33342 staining demonstrates FLIP Snitrosylation modulates apoptotic sensitivity.

4. Coimmunoprecipitation and confocal microscopy experiments demonstrating S-nitrosylation of FLIP modulates its interaction with RIP1 and localization of RIP to the cellular membrane.

5. Coimmunoprecipitation and confocal microscopy experiments demonstrating S-nitrosylation of FLIP does not modulate TRAF2 binding to FLIP and is not dependent on FLIP S-nitrosylation status.

6. Examination of protein processing of FLIP following death receptor ligation demonstrating S-nitrosylation of FLIP promotes protein processing into shorter forms. Results shown in Western blot. 


\section{List of Figures}

\section{Chapter 3. Study 2}

1. Graphical representation of how ROS regulate migration of human lung epithelial $\mathrm{H} 460$ cells and correlating MTT viability assay. Results indicate $\mathrm{OH}$. is promoting and $\mathrm{O}_{2}$ and $\mathrm{H}_{2} \mathrm{O}_{2}$ are inhibitory to migration in $\mathrm{H} 460$ cells during wound healing.

2. DCF fluorescence and electron spin resonance experiments and verifying ROS modulators effect on cellular ROS in $\mathrm{H} 460$ cells.

3. The effects of overexpression of Cav-1 on relative migration and Transwell invasion in $\mathrm{H} 460$ cells shown by Western blot, bright-field microscopy and graphically represented. The effects of Cav-1 knockdown by shRNA on relative migration and invasion in $\mathrm{H} 460$ cells shown by Western blot, bright field microscopy and graphically represented. Results indicate Cav-1 expression is directly correlated with migration and invasion in $\mathrm{H} 460$ cells.

4. Effects of Cav-1 knockdown by shRNA on ROS-modulated cell migration, invasion shown by graphical representation only. Results indicate ROS mediated migration occurs through Cav-1. Additionally Cav-1 knockdown by shRNA or ROS downregulates phospho-Akt shown by Western blot and graphical representation.

5. Experiments investigating ROS regulation on Cav-1 expression by Western blot and graphical representation. Results indicate $\mathrm{O}_{2}$ and $\mathrm{H}_{2} \mathrm{O}_{2}$ inhibit Cav-1 expression. Conversely, $\mathrm{OH}$. upregulates Cav-1 expression.

6. Western blots, bright-field microscopy of Transwell migration assays and graphical representation show dose dependent effects of ROS on Cav-1 expression, cell migration and invasion.

7. RT-PCR experiment determining ROS effect on Cav-1 mRNA expression shows no significant differences indicating ROS mediated effect is not transcriptional. Western blot and graphical representation demonstrate ROS effect on Cav-1 is through protein degradation via proteosome inhibition.

8. Western blot and bright-field microscopy assays demonstrate the effects of ROS modulators on cell migration and invasion in Cav-1 overexpressing H460 cells is the same as G361 melanoma cells. 


\section{Glossary}

\begin{tabular}{|c|c|}
\hline A & Alanine \\
\hline AG & Amino-guanidine \\
\hline Bcl-2 & B-cell lymphoma 2 \\
\hline C & Cysteine \\
\hline CAT & Catalase \\
\hline Cav-1 & Caveolin-1 \\
\hline CFP & Cyan fluorescent protein \\
\hline DAPI & 4',6-Diamidino-2-phenylindole \\
\hline DCF & 2',7'-Dichlorofluorescin \\
\hline DD & Death domain \\
\hline DED & Death effector domains \\
\hline DFX & Deferoxamine \\
\hline DISC & Death inducing signaling complex \\
\hline DMNQ & 2,3-Dimethoxy-1,4-naphthoquinone \\
\hline DMPO & 5,5-Dimethyl-1-pyrroline $\mathrm{N}$-oxide \\
\hline DMSO & Dimetheyl sulfoxide \\
\hline DNA & Deoxyribonucleic acid \\
\hline DR & Death receptor \\
\hline $\mathrm{ECL}$ & Enhanced chemiluminesence \\
\hline ESR & Electron spin resonance \\
\hline EV & Empty vector \\
\hline FADD & Fas-associated protein with death domain \\
\hline FasL & Fas ligand \\
\hline FBS & Fetal bovine serum \\
\hline $\mathrm{FeSO}_{4}$ & Ferrous sulfate \\
\hline FITC & Fluorescein isothiocyanate \\
\hline FLICE & Fas-associated protein with DD-like IL-1beta-converting enzyme \\
\hline FLIP & FLICE inhibitory protein \\
\hline FLIP 2CM & FLIP double cysteine mutant \\
\hline GAPDH & Glyceraldehyde-3-phosphate dehydrogenase \\
\hline GFP & Green fluorescent protein \\
\hline $\mathrm{H}_{2} \mathrm{DCFDA}$ & 2',7'-Dichlorodihydrofluorescein diacetate \\
\hline $\mathrm{H}_{2} \mathrm{O}_{2}$ & Hydrogen peroxide \\
\hline HEK & Human embryonic kidney \\
\hline HRP & Horseradish peroxidase \\
\hline IAP & Inhibitor of apoptosis \\
\hline IKK & Inhibitor kappa B kinase \\
\hline $\mathrm{kDa}$ & kilodalton \\
\hline KO & Knock-out \\
\hline
\end{tabular}




\begin{tabular}{|c|c|}
\hline MAPK & Mitogen-activated protein kinase \\
\hline MEFs & Mouse embryonic fibroblasts \\
\hline MMP & Matrix metalloprotease \\
\hline MnSOD & Manganese superoxide dismutase \\
\hline MnTBAP & Mn(III)tetrakis(4-benzoic acid)porphyrin chloride \\
\hline mRNA & Messenger ribonucleic acid \\
\hline MTT & 3-(4,5-Dimethylthiazol-2-yl)-2,5-diphenyltetrazolium bromide \\
\hline NaFM & Sodium formate \\
\hline$N F-\kappa B$ & Nuclear factor-kappa B \\
\hline $\mathrm{nm}$ & Nanometers \\
\hline NO & Nitric oxide \\
\hline NONO & DPTA NONOate \\
\hline NSCLC & Non-small cell lung cancer \\
\hline $\mathrm{O}_{2}^{-}$ & Superoxide anion \\
\hline $\mathrm{OH}$. & Hydroxyl radical \\
\hline PBS & Phosphate buffered saline \\
\hline PI3K & Phosphatidylinositol 3-kinases \\
\hline PTIO & $\begin{array}{l}\text { 2-(4-Carboxyphenyl)-4,4,5,5-tetramethylimidazoline-1-oxyl-3-oxide } \\
\text { potassium salt }\end{array}$ \\
\hline PTM & Posttranslational modification \\
\hline RIP1 & Receptor interacting protein 1 \\
\hline RNAi & Ribonucleic acid interference \\
\hline RNS & Reactive nitrogen species \\
\hline ROS & Reactive oxygen species \\
\hline SCLC & Small cell lung cancer \\
\hline sFASL & Soluble Fas ligand \\
\hline shRNA & Short hairpin ribonucleic acid \\
\hline siRNA & Small interfering ribonucleic acid \\
\hline SNO & S-nitrosothiol \\
\hline SNP & Sodium nitroprusside \\
\hline SOD & Superoxide dismutase \\
\hline TNF-alpha & Tumor necrosis factor alpha \\
\hline TNFR & Tumor necrosis factor receptor \\
\hline TRADD & Tumor necrosis factor receptor type 1-associated death domain protein \\
\hline TRAF2 & TNF receptor-associated factor 2 \\
\hline TRAIL & TNF-related apoptosis-inducing ligand \\
\hline WT & Wild-type \\
\hline
\end{tabular}




\section{Chapter 1. Literature Review}

\section{Cancer as a NO and ROS signaling disease}

Cancer is a class of diseases that are characterized by normal cells undergoing malignant transformation. These diseases are commonly lethal to the carriers with vast differences in prognosis due to the immense disparity in genetic profiles of the many types of cancer. Cancer development is often described in three classically distinct categories: initiation, promotion and progression. This classical view dissects the disease into pre-cancerous hyperproliferative growth (initiation), cancerous development and growth (promotion) and metastasis of tumor cells and advancement of disease (progression). The development of each of these stages is the result of specific changes in the normal cellular signaling profiles resulting in aberrant cellular behavior. Overall, this aberrant behavior can be more specifically subcategorized into six areas of protein signaling that were characterized by Hanahan and Weinberg in 2000. These six categories have been widely accepted throughout the molecular cancer biology field to describe the type of changes a normal cell must make in order to undergo malignant transformation. Study 1 in this dissertation addresses two of Hanahan and Weinberg's categories; the ability of cells to: circumvent cell death and promote cell growth through examination of a potent anti-apoptotic protein in the Death Receptor pathway. Study 2 in this dissertation addresses a third category: how cells gain 
motility and invade surrounding tissues via examination of how Reactive Oxygen Species alter Caveolin-1-dependent migration. Overall, the events that prompt tumorigenesis can be largely environmental including infection, toxin exposure, radiation and other cellular stressors such as oxidative stress.

The reductive/oxidative (redox) balance in signaling function has become increasingly important in the context of disease since the pathological microenvironment and cellular homeostasis readily has imbalances. Changes in the normal redox state of the cell can damage proteins, lipids and DNA. Chemical species derived from oxygen (reactive oxygen species) and nitrogen (reactive nitrogen species) are examples of molecules that can perturb the cellular redox balance leading to oxidative and nitrosative stress. Reactive oxygen species (ROS) and nitric oxide (NO) that are readily found throughout the cell in low concentrations are vital for cellular processes. Several cellular signaling pathways have been shown to be regulated by redox status (Kamata and Hirata 1999; D'Autreaux and Toledano 2007; Hancock 2009); however at high concentrations, both ROS and NO surpass threshold toxicity leading to cell death and abnormality.

\section{$\underline{\text { Reactive Oxygen Species }}$}

ROS are naturally found in the cell as a result of cellular metabolism and respiration (Casteilla, Rigoulet et al. 2001). Superoxide anion $\left(\mathrm{O}_{2}{ }^{-}\right)$is a byproduct of oxidative metabolism that is scavenged by the superoxide dismutase (SOD) family to produce another ROS, hydrogen peroxide $\left(\mathrm{H}_{2} \mathrm{O}_{2}\right)$, in 
both the mitochondria and cytosol. $\mathrm{H}_{2} \mathrm{O}_{2}$ can additionally participate in the Fenton reaction resulting in the generation of one of the most cellular damaging ROS, hydroxyl radical (OH.) (Goldstein, Meyerstein et al. 1993; Galaris, Skiada et al. 2008). The mitochondria are the primary site for ROS generation and therefore are equipped with many catabolizing enzymes (such as MnSOD) to manage levels of ROS. Mitochondria are not the only source for ROS. NADH oxidases, cyclooxygenase, xanthine oxidase, cytochrome P450s and peroxisomes are also responsible for ROS production (Ignarro 2000; Salganik 2001).

When the generation of cellular ROS exceeds the capacities of catabolizing enzymes and electron-donating antioxidants, they can damage surrounding proteins, lipids and DNA by oxidation (Barbouti, Doulias et al. 2002; Sallmyr, Fan et al. 2008). This damage can result in cell cycle arrest (Yang, Wu et al.), apoptosis (Simon, Haj-Yehia et al. 2000) and the development of disease (Lambeth 2007). Elevated levels of oxidation have long been reported in numerous diseases including cardiovascular disease, neurodegenerative disorders and cancer. NO and ROS are widely considered in terms of tissue and cellular damage. They have recently been noted for their participation in specific protein signaling within the cell. ROS can aid in growth factor, cytokine and hormone signaling (Lambeth 2007) as well as in cellular processes of adhesion, movement (Ushio-Fukai 2006), apoptosis (Wong, Iskandar et al.) and gene transcription (Dhar, Young et al. 2002). Because ROS can promote genetic instability through DNA oxidation and it is reported to be involved in multiple 
signaling pathways, it is not surprising that elevated levels of ROS are associated with an increased risk in cancer development (Toyokuni 2004; Gius and Spitz 2006). Additionally, unrelenting cell growth can result in tumor development that exceeds the capacity of existing vasculature resulting in nutrient and oxygen shortages that put the cell under selective pressures. Hypoxic conditions alone can lead to transcriptional changes and overall increases in ROS (Kim and Chung 2007). Multiple contributing factors such as increased metabolic activity and mitochondrial dysfunction (Carew and Huang 2002; Pelicano, Xu et al. 2006) can all contribute to the overabundance of ROS seen in pathology (see figure 1). The high level of ROS seen in cancer has been pursued as a mechanism to specifically target tumor cells for cell death. Because cancer cells have elevated levels of ROS they have a high dependence on ROS-eliminating agents. Zhou et al. showed that 2-methoxyestradiol induces ROS-mediated apoptosis in leukemia cells by inhibiting SOD (Zhou, Hileman et al. 2003). Additionally, tumor cells are reported to have high innate ROS levels (Burdon 1995) making them vulnerable to toxic thresholds, therefore additional ROS insults induced by exogenous agents can promote cell death in tumor cells. For example, chemotherapeutic agent, bortezomib induces apoptosis via ROS generation in NSCLC cells and is currently used in the treatment of multiple myeloma (Ling, Liebes et al. 2003). One potential problem with these prooxidant treatments is the oversight of ROS effect on protein signaling at sublethal levels. Since it has been established that ROS can implement signaling changes in targeted molecules these types of therapies could have ample 
unwanted effects. Additionally, due to the inherent oncogenic nature of ROS, treatments that increase overall ROS levels should be proceeded with caution.

The alternative approach of antioxidant therapies for cancer has demonstrated success in anticancer treatments. Anitoxidant treatments of vitamin $\mathrm{E}$ and pyrrolidone dithiocarbamate in combination with chemotherapeutics 5-fluorouracil and doxorubicin were shown to induce apoptosis in colorectal cancer in vivo (Chinery, Brockman et al. 1997). Vitamin E decreases proliferation in cervical, lung and ovarian carcinoma (Jha, Bedford et al. 1999). Enhancement of ROS clearance also shows similar results. For example, the overexpression of SOD inhibits cell growth in breast cancer (Weydert, Waugh et al. 2006). Basal ROS levels are heterogenous among the human population suggesting that although antioxidant therapy may be advantageous for patients with a high level of ROS, it may be detrimental to people with a low level of ROS by decreasing the activity of ROS-dependent protective mechanisms (Salganik 2001). Production of ROS is essential for a number of biochemical reactions and modulating cellular processes such as antimicrobial phagocytosis and apoptosis (Johnson, $\mathrm{Yu}$ et al. 1996). $\mathrm{H}_{2} \mathrm{O}_{2}$ and $\mathrm{O}_{2}{ }^{-}$are indicated to play a crucial role in mechanisms underlying proliferative responses via transcription factors: c-fos, c-myc and NF-kB (Burdon 1995). Additionally, antioxidant therapies have been reported to inhibit the specific therapeutic activity of anticancer drugs in patients (Labriola and Livingston 1999). Much of the evidence that antioxidant therapy can be anti-tumorigenic has been done in the context of healthy individuals where ROS profiles are considerably 
lower than in cancer (Seifried, McDonald et al. 2003). A more detailed understanding of how ROS modulates cancer protein signaling is necessary to better assess whether pro- or antioxidant therapy is beneficial.

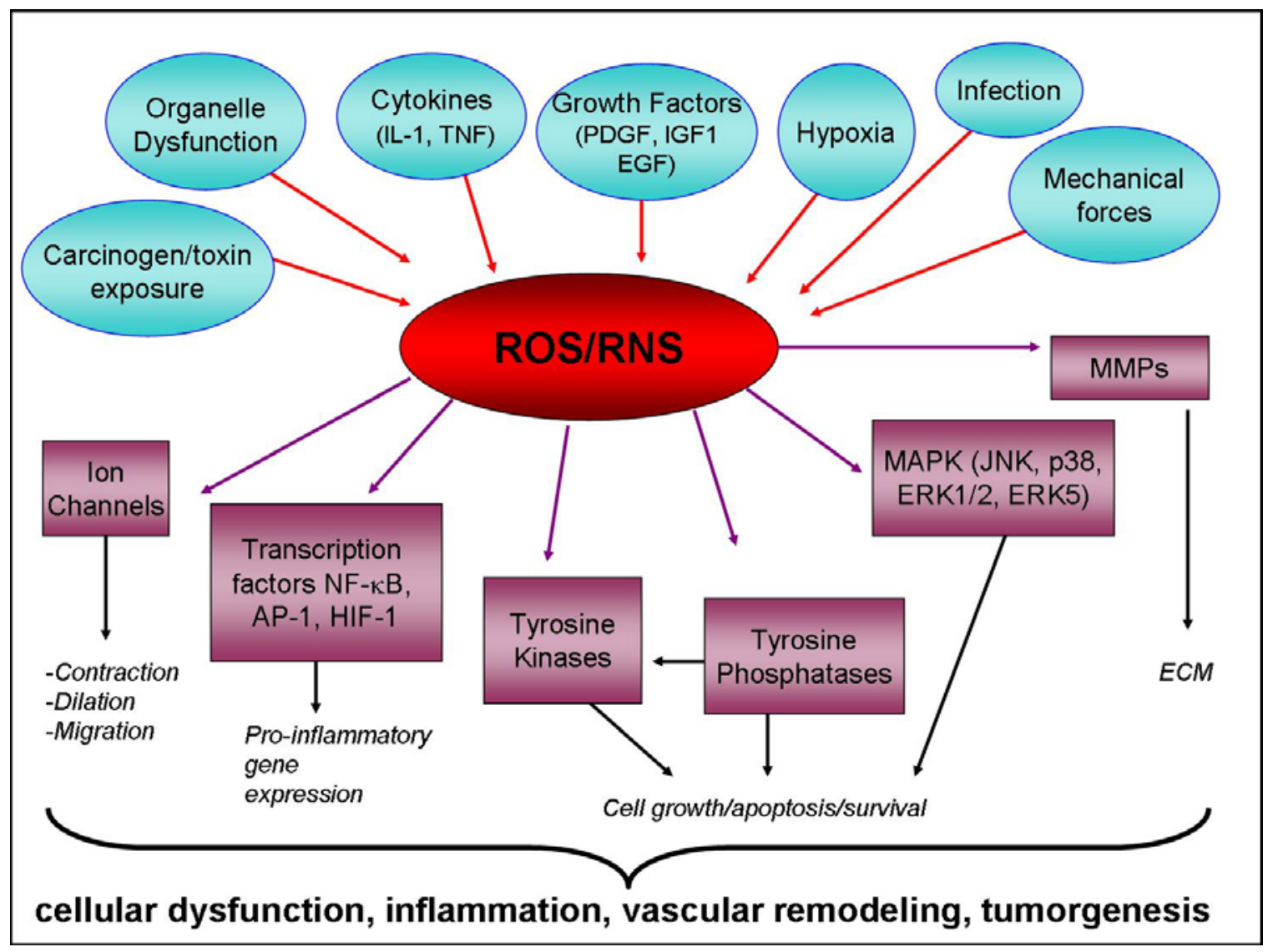

Figure 1. Multiple stimuli lead to the production of ROS and RNS which mediate many redox-dependent signaling pathways. ROS/RNS have been noted to induce MAPK activity and tyrosine kinases (i.e. src, Ras, PI3K) and inhibit tyrosine phosphatases. They can also enhance transcription factor activity. ROS can stimulate ion channels (i.e. plasma membrane $\mathrm{Ca}^{2+}$ and $\mathrm{K}^{+}$) leading to overall changes in cation balance. Adapted from (Pelicano, Carney et al. 2004; Aruoma, Grootveld et al. 2006).

\section{Nitric Oxide}


NO is primarily produced intracellularly from L-arginine by a family of four nitric oxide synthases (NOS) that are differentially expressed. Neuronal NOS (nNOS or NOS1) is constitutively expressed and involved in cell communication. Inducible NOS (iNOS or NOS2) can be induced by lipopolysaccharides (LPS), cytokines, and other immunomodulatory compounds and its expression is modulated by proinflammatory transcription factor nuclear factor kappa B (NF$\kappa \mathrm{B})$ and the mitogen-activated protein kinase (MAPK) pathway (Lowenstein and Padalko 2004). It is the only family member that has calcium-independent activity and is the most frequent isoform associated with tumorigenesis. Endothelial NOS (eNOS or NOS3) is constitutively expressed in many cell types and generates $\mathrm{NO}$ in blood vessels to regulate vascular function. eNOS produces lower levels of NO than iNOS, however, it has been a suggested target in cancer therapy due to its role in angiogenesis, mobility and invasiveness (Ying and Hofseth 2007). Mitochondrial NOS (mtNOS) is thought to be a variant of nNOS and contributes to redox-dependent signaling in the mitochondria (Finocchietto, Franco et al. 2009).

$\mathrm{NO}$ is a highly diffusible pleiotropic molecule that is short lived and therefore exhibits localized effects. The direct interaction of NO with a target protein occurs via formation of a disulfide bond with a cysteine residue on the target protein. Nitrosylation (or nitrosation) of a target protein can alter its function and interactions; therefore it is a post-translational modification regulating protein function. These effects can be either activating or inhibitory. The knowledge of how many proteins are sensitive to NO signaling only became 
known after Jaffrey and Snyder developed the Biotin Switch Method in 2001 (Jaffrey and Snyder 2001). NO has already been reported to react with more than a hundred proteins to form S-nitrosothiols (SNO) (Paige, Xu et al. 2008).

\section{S-nitrosylation}

The posttranslational modification, S-nitrosylation, is considered to be selective and specific; although, the regulation of the SNO system is not yet well understood. It has been proposed that proximity of iNOS to a target protein regulates the ability for it to be nitrosylated (Bredt 2003). For example, cyclooxygenase-2 has been shown to be S-nitrosylated after coimmunoprecipitation with iNOS following inflammatory stimulation (Kim, Huri et al. 2005). Alternatively, Mitchell et al. demonstrated thioredoxin transnitrosylates caspase-3 through domain recognition; thereby regulating caspase activity (Mitchell and Marletta 2005). This type of protein-protein interaction with SNO could mimic the specificity of $\mathrm{SH} 2$ domains within the context of tyrosine phosphorylation (Tannenbaum and White 2006). This is not the first time that thioredoxin has been shown to have a role in SNO. Thioredoxin was first reported to act as a protein disulphide reductase in plants (Schurmann and Jacquot 2000). More recently, S-nitrosoglutathione reductase (GSNOR), an enzyme ubiquitously expressed, has emerged as an important factor in governing the S-nitrosylation of SNO proteins (Benhar, Forrester et al. 2009). The increasing number of reports describing enzymes involved in oxidative 
signaling further indicates the specificity and importance of NO in protein signaling.

NO has been widely attributed to promote tumor formation and metastasis (Fukumura, Kashiwagi et al. 2006). NO has been reported to interact with HIF$1 \alpha$ to regulate metastasis, invasion and chemoresistance in human gastric and breast cancer cells (Maccarrone and Brune 2009). Additionally, increases in iNOS activity are definitively associated with tumorigenesis in many types of cancer (Jenkins, Charles et al. 1995; Crowell, Steele et al. 2003) and numerous selective inhibitors of iNOS show a reduction in tumor growth (Thomsen, Scott et al. 1997). Elevated iNOS expression is reported in tumor cells (Siedlar, Mytar et al. 1999; Takahashi, Kitahashi et al. 2008) and in the tumor microenvironment (Hussain, He et al. 2008). The high levels of NO produced by iNOS are normally used by macrophages for antimicrobial phagocytosis (Babior 1978; Nussler, Di Silvio et al. 1992), however tumor-associated macrophages can be part of a chronic inflammatory microenvironment that promotes tumor growth rather than targeting the cells for death. In contrast, there have been some reports that high levels of NO and iNOS overexpression suppress tumorigenesis (Harada, Supriatno et al. 2004). However, Edwards et al. reported that NO inhibits cell growth in vitro but stimulates tumorigenesis and metastasis in vivo (Edwards, Cendan et al. 1996) potentially explaining the discrepancies between pro- and anti-cancer effects. Additionally, in vitro experiments fail to incorporate any paracrine effects from the stroma which contains cells that both express iNOS 
and are responsive to NO and inflammatory stimuli thereby representing a different environment.

\section{$\underline{\text { Reactive Nitrogen Species }}$}

RNS are a family of molecules that are generally derived from NO; for example, $\mathrm{O}_{2}^{-}$and $\mathrm{NO}$ react rapidly to form peroxynitrite (ONOO${ }^{-}$) (Wiseman and Halliwell 1996). Although RNS such as $\mathrm{ONOO}^{-}$and dinitrogen trioxide $\left(\mathrm{N}_{2} \mathrm{O}_{3}\right)$ are more commonly noted for toxicity they also demonstrate effects on specific signaling pathways. Wu et al. demonstrated RNS treatment leads to JNKmediated non-apoptotic cell death (Wu, Zhang et al. 2008). Cells that are recruited to sites of injury and infection secrete high levels of NO leading to an overall increase in RNS (Patel, McAndrew et al. 1999). Like ROS, RNS have also been shown to moderate growth factor, cytokine and environmental stress signaling (Lander 1997; Suzuki, Forman et al. 1997).

During chronic inflammation, activated inflammatory cells release RNS and ROS that can promote tumorigenesis in the initiated cell. The complexity of ROS and NO effects on cellular fate lies both in the signaling potential as well as the cumulative total dose and cell type (Kawanishi, Hiraku et al. 2006). Therefore, understanding how ROS and RNS mediate changes in protein signaling and which cellular pathways involved in cancer are affected is increasingly important. 


\section{Death Receptor Signaling - Apoptotic}

Inflammation, proliferation and apoptosis are tightly regulated cellular processes that are regulated in part by a family of transmembrane receptors called Death Receptors (DR). There are at least eight members of the DR family including: Fas, Tumor Necrosis Factor Receptor 1 (TNFR1), TNFR2, DR3, DR4 (also known as TRAIL-R1), and DR5 (also known as TRAIL-R2) (Lavrik, Golks et

al. 2005). DRs are members of the TNFR superfamily which includes CD40 and lymphotoxin $\beta$ receptors that are important in immune cell signaling. In addition to the cysteine-rich extracellular domain found in all TNFR superfamily members, DRs contain a homologous cytoplasmic sequence of 80 amino acids termed the Death Domain (DD) (Tartaglia, Ayres et al. 1993). Signals are propagated by extracellular trimeric cytokines (also known as DR ligands) that bind DRs and initiate the formation of oligomeric signaling platforms such as a DISC (or Death Inducing Signaling Complex) which can lead to apoptosis.

Apoptosis is a type of cell death that is required for development and cellular homeostasis that is frequently dysregulated in disease. It was originally characterized by Kerr et al. in 1972 when cells exhibited distinct and consistent morphological changes preceding cell death. This process appeared necessary for embryogenesis, development and cell turnover (Kerr, Wyllie et al. 1972). Apoptosis can be signaled by two converging pathways: the extrinsic pathway 
and the intrinsic pathway. Both pathways are dependent on cysteine-aspartate proteases called caspases (Li and Yuan 2008). The extrinsic pathway is a ligand-mediated process that occurs through DRs such as Fas, resulting in the activation of initiator caspases (i.e., procaspase-8, procasase-10) leading ultimately to the activation of effector caspases (i.e., procaspase-3, procaspase7) and cell death. The intrinsic pathway (also known as the mitochondrial pathway) is initiated inside the cell in response to DNA damage, hypoxia, irradiation, abnormal cell cycle, changes in growth factors and other acute stress signals. The initiation of the intrinsic pathway is contingent on the balance of proand anti-apoptotic proteins of the $\mathrm{Bcl}-2$ family which maintain the integrity of the mitochondrial membrane. Cytochrome $\mathrm{c}$ and Second Mitochondria-derived Activator of Caspases (SMAC, also referred to as DIABLO) are maintained in the mitochondrion. Following stimulation of the intrinsic pathway, proapoptotic Bcl-2 proteins, Bax or Bak can insert into the mitochondrial membrane initiating Mitochondrial Outer Membrane Permeabilization (MOMP) (Chipuk, BouchierHayes et al. 2006). Proapoptotic proteins released from the mitochondrion stimulate the formation of the apoptosome complex; consisting of cytochrome c clustered with Apaf-1 and caspase-9 (Zou, Li et al. 1999). Simultaneously, SMAC/DIABLO bind and inhibit cellular Inhibitors of Apoptosis Proteins (CIAP) in the cytosol that negatively regulate caspase- 9 under resting conditions. The mitochondrial release of pro-apoptotic factors is considered an irreversible signal for cell death (Green and Amarante-Mendes 1998). The final stage of apoptosis via both pathways involves chromatin condensation, DNA fragmentation, cell 
shrinkage and membrane blebbing. This phenotype is distinctive of apoptosis and unlike other types of cell death, such as necrosis and autophagy.

$\underline{\text { Fas }}$

Fas (also known as CD95 and Apo-1) is the most classically studied DR. It was identified in 1989 (Trauth, Klas et al. 1989) and immediately recognized for its importance in T and B cell homeostasis (Ju, Panka et al. 1995; Krammer 2000). Defective Fas signaling is involved in Autoimmune Lymphoproliferative Syndrome (ALPS) (Fisher, Rosenberg et al. 1995), Graft-versus-Host Disease (GVHD) (Via, Nguyen et al. 1996) and many types of cancer (Houston and O'Connell 2004).

Fas receptor is activated and trimerizes when bound by Fas Ligand (FasL) leading to conformational changes of the cytoplasmic tail of the protein. Figure 2 illustrates conventional signaling of how Fas commonly signals for apoptosis through formation of a DISC. Fas can also form signaling complexes that result in proliferation and inflammation (Wajant 2002). Fas-Associated protein with Death Domain (FADD) is an adaptor molecule that is recruited to the cytoplasmic tail of Fas through Death Domain (DD) homotypic interaction following receptor ligation (Chinnaiyan, O'Rourke et al. 1995). FADD then recruits effector proteins such as caspase-8, caspase-10 and FLICE-inhibitory protein (FLIP) through binding of Death Effector Domain (DED) interactions. FADD is recruited for classical apoptotic signaling. When two or more molecules of procaspase-8 bind FADD to 
form the DISC, they are catalytically cleaved by autoactivation (Chang, Xing et al. 2003) leading to initiation of the caspase cascade and subsequent apoptosis.

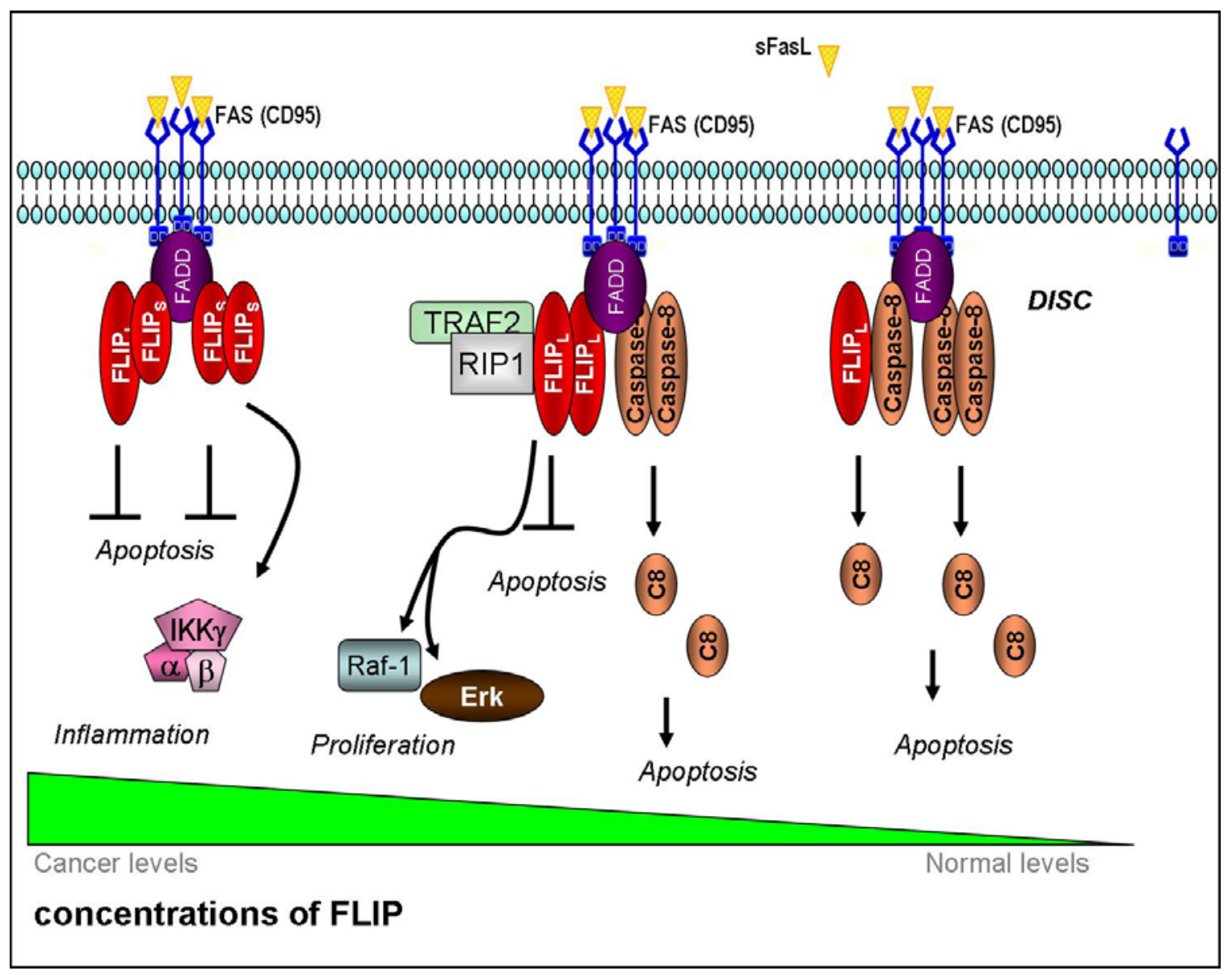

Figure 2. FLIP concentration alters DISC-dependent apoptosis, inflammation and proliferation signaling through other complexes. When highly expressed, FLIP competes with caspase-8 for FADD binding at the DISC preventing activation of the apoptotic caspase cascade. Adapted from (Mathew and White 2006; Lamkanfi, Festjens et al. 2007).

Most Cells are categorized into two types based on their response to Fas ligation and DISC assembly (Scaffidi, Fulda et al. 1998). In type I cells, Fasmediated caspase- 8 activation of caspase- 3 is sufficient for inducing cell death. Fas has been reported to cluster in lipid rafts providing ample oligomerization to 
robustly activate caspase-8 (Muppidi and Siegel 2004). In type II cells, cell death requires amplification of the apoptotic signal which requires mitochondrial involvement. In these cells, caspase-8 cleaves Bid which inactivates antiapoptotic protein $\mathrm{Bcl}-2$ and destabilizes the mitochondrial membrane leading to the release of cytochrome $\mathrm{c}$ and formation of the apoptosome. Both cell types share the same apoptotic result through final activation of effector caspase-3.

\section{$\underline{F L I P}$}

FLIP is another DED-containing protein that is an integral component of the DISC. Cellular FLIP is expressed in mammalian cells as two splice variants, FLIP short $\left(F_{L I P S}\right)$ and FLIP long $\left(F L I P_{L}\right)$. Both FLIPS and FLIP contain two Nterminal DEDs. FLIP $\mathrm{L}$ contains the additional C-terminal Caspase-Like Domain (CLD). FLIP $P_{L}$ is structurally homologous to caspase-8 with the exception of an active cysteine residue in caspase- 8 substituted by a tyrosine residue, lending it proteolytically inactive to cleave caspase substrates (Irmler, Thome et al. 1997). Viral expressed FLIP (vFLIP) consist of only the two N-terminal DEDs (homologous to cFLIPS) and are expressed by $\gamma$-herpesviruses and the tumorigenic molluscipoxvirus, (Thome, Schneider et al. 1997).

FLIP is most notably an inhibitor of apoptosis and is implicated in many types of cancer resistant to FasL-mediated apoptosis (Irmler, Thome et al. 1997). FLIP $L$ has been implicated as both a pro- and antiapoptotic protein at the DISC. At high concentrations, FLIP $L$ competes with procaspase-8 for FADD binding at the DISC thereby preventing all autoprocessing and caspase activation. At low 
and physiological levels, FLIP $\mathrm{L}$ can promote apoptosis when at the DISC through allosteric interactions with procaspase 8 (see figure 2) (Scaffidi, Schmitz et al. 1999; Micheau, Thome et al. 2002). Additionally, the different isoforms of FLIP have been reported to have distinct protein-protein interactions and signaling capacities. FLIP $L$ can activate Raf1 and Erk and is shown to recruit TRAF1, TRAF2 and RIP to Fas complexes (see figure 2) (Kataoka, Budd et al. 2000). FLIP $L$ also contains a Nuclear Localization Sequence (NLS) which mediates interaction with MAPK-independent AP-1 activation (Zhang, Chen et al. 2009).

FLIP has been shown to be a substrate of caspase-8 and cleaved at D376 which promotes TRAF2 binding (Kataoka and Tschopp 2004) and RIP1 recruitment to the DR membrane complex (Dohrman, Kataoka et al. 2005). FLIP $L$ has also been reported to be cleaved at $\mathrm{D} 198$, generating a protein fragment of only the two N-terminal DEDs, equivalent to FLIP that activates NFкB (Golks, Brenner et al. 2006). There is good indication that vFLIP also activates NF-KB through interactions with IKK $\gamma$, supported by molecular modeling studies (Bagneris, Ageichik et al. 2008).

$\underline{T R A I L}$

TNF-related apoptosis-inducing ligand (TRAIL, also known as Apo-2L) is a cytokine in the TNF superfamily that binds DRs: TRAIL Receptor 1 (TRAIL-R1, also known as DR4) and TRAIL-R2 (also known as DR5). It also binds Decoy Receptor 1 (DcR1), Decoy Receptor 2 (DcR2) and osteoprotegerin (OPG). DcR1 lacks a cytoplasmic domain preventing propagation of a cellular signal and 
DcR2 has a truncated cytoplasmic domain which leads to incomplete activation. OPG is a soluble and possible additional decoy receptor (Emery, McDonnell et al. 1998). TRAIL induces apoptosis similarly to FasL stimulation (Pitti, Marsters et al. 1996) and is constitutively expressed in numerous tissues (Wiley, Schooley et al. 1995). TRAIL ligation of TRAILR1/R2 results in formation of a DISC. TRAIL apoptotic signaling also involves mitochondria apoptotic involvement through Bid signaling (Werner, de Vries et al. 2002).

\section{Non-apoptotic Death Receptor Signaling}

\section{$\underline{T N F R 1 / 2}$}

Not all DRs primarily signal for cell death (Budd 2002). DRs can signal for cell survival, differentiation, inflammation and proliferation through pathways involving NF-kB, Jun N-terminal Kinase (JNK), MAPK and p38 MAPK (Aggarwal 2003). The most distinguished DRs that promote inflammation and proliferation are TNFR1 and TNFR2. TNFR1 (also known as p55) is ubiquitously expressed across most cell types, whereas TNFR2 (also known as p75) is primarily expressed on haemopoietic cells (Chen, Subleski et al. 2008). Their cognate ligand, TNF- $\alpha$, is a $17 \mathrm{kDa}$ potent inflammatory cytokine that is secreted by macrophages, neutrophils, T- and NK-cells following their infection as part of an inflammatory immune response or as an acute stress response to facilitate tissue repair (Balkwill 2002). 
Following receptor ligation, TNFR1 leads to the formation of two distinct signaling complexes (see figure 3). Complex I is rapidly formed at the membrane consisting of TNFR1-Associated Death Domain (TRADD), TNF receptorassociated factor 2 (TRAF2), Receptor Interacting Protein 1 (RIP1) and cIAP1. This complex leads to pro-survival responses in the cell through NF-KB and MAPK signaling (Micheau and Tschopp 2003). Complex II subsequently forms when RIP1, TRAF2 and TRADD disassociate from TNFR1 (and the membrane) and either RIP1 or TRADD recruit FADD and/or procaspase-8 through DED homotypic interaction leading to DISC formation and subsequent apoptosis ( $\mathrm{Li}$ and Yuan 2008) (Micheau and Tschopp 2003). TNF- $\alpha$ ligation results in Complex I formation within 2 minutes (Natoli and Austenaa 2008) and only leads to Complex II formation after prolonged signaling (+24 hours). In the formation of Complex II, clAP1/2 are autoubiquitinated and degraded resulting in the deubiquitination of RIP1, thereby allowing DISC recruitment (Nastiuk and Krolewski 2008). 


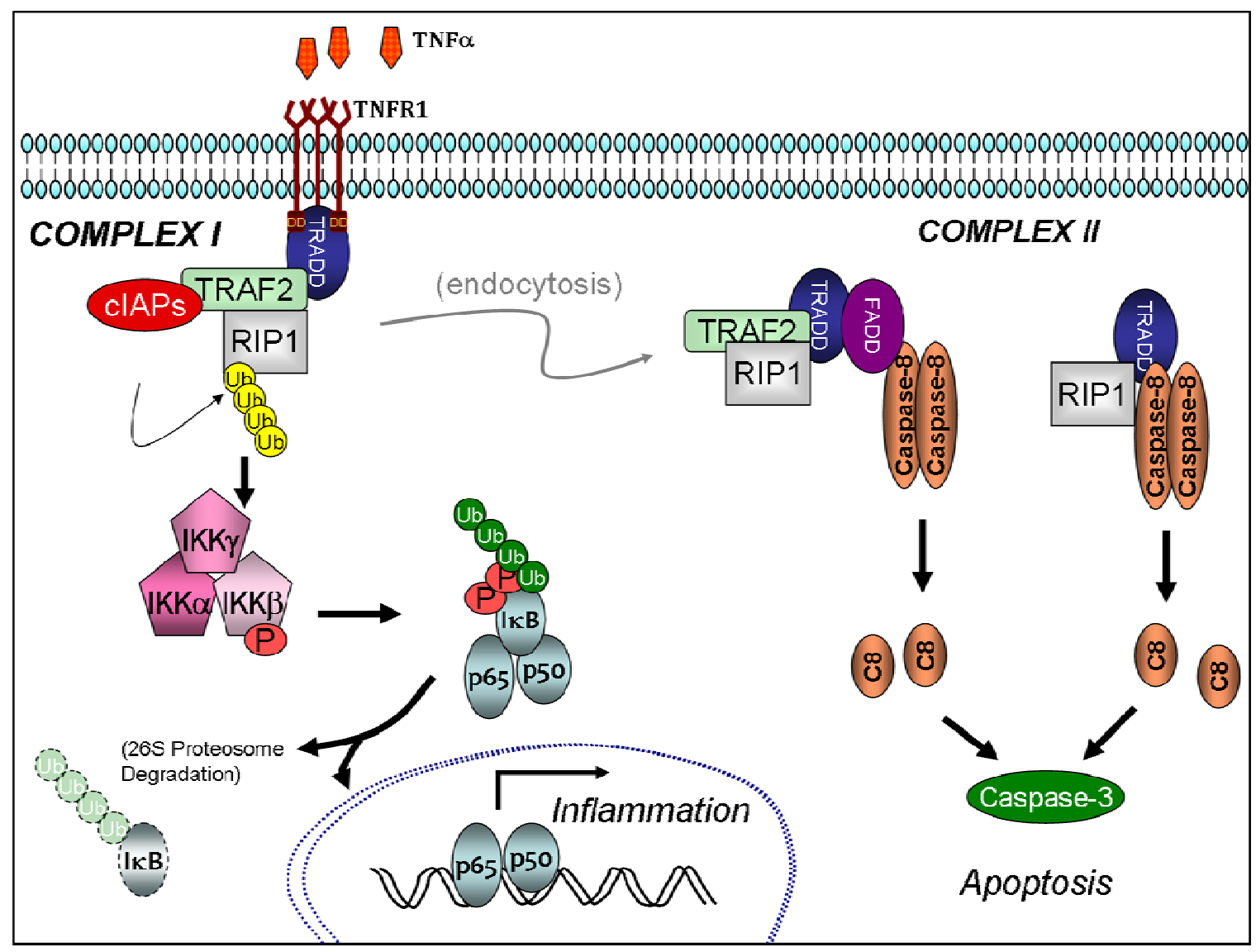

Figure 3. TNFR1/2 signaling results in two complexes. Complex I, assembled instantly following TNF- $\alpha$ ligation results in non-degradative ubiquitination chains linked at lysine 63 (illustrated in yellow) attached to RIP1 and results in downstream degradative ubiquitin chains linked at lysine 48 (illustrated in green) attached to phosphorylated IкB. Adapted from (Silke and Brink; Wertz and Dixit).

TNFR1 classically signals for activation of the potent proinflammatory transcription factor NF-kB via complex I through TRAF2 and RIP1. Both these proteins have been reported to interact with components of the IKK complex

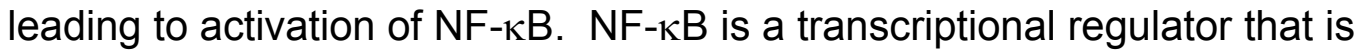
activated by various stimuli including cytokines (such as TNF- $\alpha$ ), chemokines, adhesion molecules and immune responses to regulate inflammation, cell 
survival and proliferation. NF-kB transcription factors are homo- or heterodimers of the Rel family proteins that bind specific DNA sequences called $\kappa \mathrm{B}$ sites in the promoter region of target genes. Most NF- $\kappa \mathrm{B}$ dimers contain RelA (p65) or c-Rel and are regulated by Inhibitor of kappa B (lкB) proteins.

$\underline{N F-\kappa B}$

NF- $\kappa \mathrm{B}$ can be activated via the canonical or non-canonical pathway.

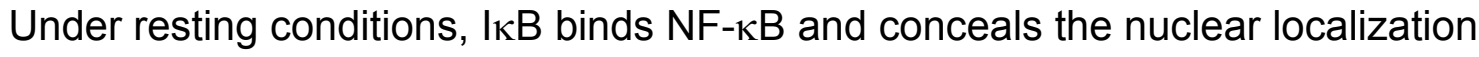
sequence resulting in cytosol sequestration. TNFR1/2 ligation classically leads to canonical activation of NF-kB. Figure 3 illustrates when TNF- $\alpha$ binds TNFR1, TRADD is recruited to the receptor which binds TRAF2 and RIP1. Together this leads to the activation of $I_{\kappa} B$ kinase (IKK) complex which phosphorylates $I_{\kappa} B$. The phosphorylated $I_{\kappa} B$ is ubiquitinated and degraded by the proteosome allowing a free NF-kB to translocates into the nucleus to function as a transcription factor upregulating numerous target genes. The IKK complex contains three subunits, IKK $\alpha, \operatorname{IKK} \beta$, IKK $\delta$ or NEMO. The $\alpha$ and $\beta$ subunits contain kinase activity to phosphorylate $I_{\kappa} B$. NEMO is the regulatory subunit mediating binding to upstream activators of IKK $\alpha$ and IKK $\beta$. Recently IKK

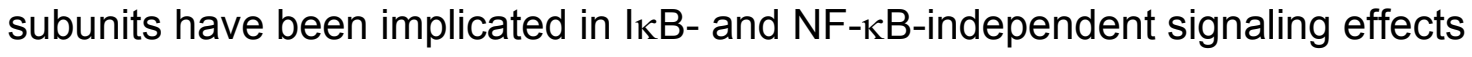
(Perkins 2007) indicating IKK activation effects may be more widespread than initially thought. The non-canonical activation of NF-kB is only stimulated by a 
small number of stimuli and results in NF-kB Inducing Kinase (NIK) to activate IKK $\alpha$.

Canonical signaling depends on two proteins upstream of IKK activation TRAF2 and RIP1. Classically it has been reported that TRAF2 is necessary to recruit NEMO to the receptor complex and RIP1 is necessary for IKK activation, giving each a distinctive role in NF-kB activation (Devin, Cook et al. 2000).

$\underline{\text { TRAF2 }}$

TRAF2 is a member of the TRAF protein family, which are all intermediates in NF-kB signaling. TRAFs have a 150 amino acid TRAF domain in the C-terminus which enables oligomerization between TRAFs and facilitates receptor binding. TRAFs 2-7 have a RING domain near the $\mathrm{N}$-terminus enabling them to exhibit ubiquitin ligase activity. TRAF2 is the most extensively studied family member and its overexpression has been correlated with invasiveness and poor prognosis of cancer patients (Trauzold, Roder et al. 2005; van Galen, Muris et al. 2008). Although TRAF2 dependent NF-kB activation has been clearly demonstrated, TRAF2 knockout mice have deficient JNK signaling but still demonstrate proper NF-kB activation (Yeh, Shahinian et al. 1997). Tada et al. discovered TRAF2/TRAF5 double knockout mice had impaired NF-kB signaling (Tada, Okazaki et al. 2001) suggesting that TRAF5 can compensate for TRAF2 deficiencies. This type of signaling overlap is evident in multiple protein families. Interestingly, not all TRAF members promote NF-kB activation, for example, 
TRAF 3 has been reported as an inhibitor of NIK-dependent non-canonical signaling (He, Saha et al. 2007).

\section{$\underline{R I P 1}$}

RIP1 is in the RIP family proteins and is constitutively expressed in most tissues. RIP1 has a DD facilitating interactions with DD-containing proteins: DR receptors, TRADD and FADD. RIP1 has been reported to be mandatory for NFКB activation in numerous studies (Kelliher, Grimm et al. 1998; Poyet, Srinivasula et al. 2000; Jackson-Bernitsas, Ichikawa et al. 2007). It has been shown to interact with IKK $\gamma$ (NEMO) to activate IKK (Kelliher, Grimm et al. 1998). RIP can interact in both the TNFR1 prosurvival signaling complex as well as the DISC and has therefore been proposed as a link between the two. Specifically the hinging component of promoting cell proliferation or cell death is determined by RIP ubiquitination state (Festjens, Vanden Berghe et al. 2007; Declercq, Vanden Berghe et al. 2009). Following TNFR1 ligation, RIP1, TRAF2 and cIAP1/2 are recruited to the receptor complex. cIAP1/2 target RIP1 for ubiquitination with chains linked through lysine 63 (Varfolomeev, Goncharov et al. 2008). This is a non-degradative posttranslational modification that promotes complex I formation and the downstream activation of MAPK and NF-kB (see figure 3). Inhibition or degradation of cIAPs allows deubiquitination of RIP1 thereby promoting FADD or procaspase-8 binding through DED interactions, resulting in DISC formation. 


\section{Divergent Death Receptor Signaling}

Although TNFR1 is classically implicated in pro-survival and inflammatory responses, it can additionally lead to apoptosis through TRADD recruitment of FADD and subsequent assembly of a DISC (Lavrik, Golks et al. 2005) (see figure 3). This signaling is much less frequent than the inflammatory signaling (Ashkenazi and Dixit 1998). Crosstalk between receptors has also been widely reported. Fotin-Mleczek et al. reported that TNFR2 can enhance TNFR1mediated apoptotic signaling. They indicate that TNFR2 downregulates clAPs and promotes TRAF2 degradation leading to enhanced activation of caspase- 8 (Fotin-Mleczek, Henkler et al. 2002).

Other DRs and ligands signal for inflammation and proliferation aside from TNFR1/2. Fas signaling can result in caspase-8 mediated NF-kB activation through RIP1 recruitment (Shikama, Yamada et al. 2003). Fas and TRAIL-Rs are also reported to recruit the TRADD adaptor protein and activate NF-kB (Schneider, Thome et al. 1997; Tang, Wang et al. 2009). Additionally TRAIL has been reported to activate NF-kB through Protein Kinase C (Ehrhardt, Fulda et al. 2003) and induce proliferation through Akt and Erk pathways (Secchiero, Milani et al. 2003). FLIPL can recruit TRAF1, TRAF2 and RIP to the DISC (Kataoka, Budd et al. 2000) and FLIPS can bind and activate IKKY (Golks, Brenner et al. 2006) (see figure 2). Gene knockouts of any member of the DISC (FADD, caspase-8 and FLIP) are embryonically lethal due to incomplete heart development and T-cell proliferation (Kennedy, Kataoka et al. 1999; Yeh, Itie et 
al. 2000; Chun, Zheng et al. 2002) further indicating the importance of these proteins in proliferative pathways.

Many components of the DR pathway undergo PTM that mediate their function. FADD phosphorylation is required for its compartmentalization (Osborn, Sohn et al. 2007). The Fas cytosolic domain can be phosphorylated and ubiquitinated to modulate signaling (Voss, Lettau et al. 2008). Components of NF-кB have been shown to be a target of S-nitrosylation leading to inhibition of transcriptional activation (Marshall and Stamler 2001; Marshall, Hess et al. 2004). We have previously shown that FLIP can be S-nitrosylated (Chanvorachote, Nimmannit et al. 2005). Other groups have shown caspase-3 can also be S-nitrosylated leading to its inactivation of enzyme activity (Mannick, Schonhoff et al. 2001). Further understanding of the PTMs that modulate proteins in the DR pathway can potentially provide insights for future targeted therapies.

\section{Death Receptor Signaling in Disease}

Tumor cells frequently gain apoptotic resistance via mutation of the signaling proteins within either the intrinsic or extrinsic apoptotic pathways. Deficiencies in apoptosis as described by Hanahan and Weinberg is one of the hallmarks of cancer (Hanahan and Weinberg 2000) that we are primarily interested in throughout Study 1. Apoptotic resistance has also been described 
as a crucial characteristic of metastasis (Mehlen and Puisieux 2006). Mutations in the apoptotic DR-pathway further assist tumor cell survival by providing escape from immune surveillance. Specifically, many tumor cells express sFas or TRAIL to eliminate infiltrating T-cells and lymphocytes in addition to common antiapoptotic mutations within the DR-pathway (Hahne, Rimoldi et al. 1996; Stewart and Abrams 2008). Chemotherapy and radiation are conventional therapies in cancer treatment that rely on DR-pathways to induce cell death in tumor cells. Chemotherapy-resistant tumor cells are increasingly formidable since they have gained safeguards against apoptosis whereas the normal cells have not, potentially providing an environment that will allow for survival of the tumor cell.

TRAIL has emerged as a promising therapeutic target for adjuvant cancer therapies for its ability to target cancer cells for apoptosis with relatively low toxicity in normal cells (Ashkenazi, Pai et al. 1999; Falschlehner, Ganten et al. 2009). The decoy receptors are widely expressed in normal tissues and offer putative resistance to TRAIL induced apoptosis (Sheridan, Marsters et al. 1997). Additionally, many cancer types have shown elevated levels of TRAIL-R1/R2 resulting in an increased sensitivity to TRAIL-induced apoptosis (Oikonomou, Kosmidou et al. 2009). Chemotherapy and radiation treatment induces p53dependent apoptosis, however, many cancers obtain p53 mutations which provide resistance to these treatments. TRAIL signals for apoptosis regardless of p53 activation and therefore is an advantageous adjuvant therapy (Wang 2008). Recombinant TRAIL therapy induces apoptosis in many cancers 
including prostate (Yu, Mandlekar et al. 2000), breast (Keane, Ettenberg et al. 1999), glioma (Rieger, Naumann et al. 1998), multiple myeloma (Gazitt 1999) to name a few. Additionally, antibodies that stimulate TRAILR1/R2 have also shown success in vivo (Chuntharapai, Dodge et al. 2001; Ichikawa, Liu et al. 2001). Unfortunately, the success of TRAIL therapy has not been seen in all cancers.

Mutations in TRAIL-R1/R2 can translate to insensitivity to TRAIL-mediated apoptosis in metastatic breast cancer (Shin, Kim et al. 2001). Similar mutations have been reported in bladder and ovarian cancers (Kim, Fisher et al. 2000). Elevated levels of antiapoptotic proteins Bcl-2, survivin and XIAP all demonstrate TRAIL-mediated apoptotic resistance (Chawla-Sarkar, Bae et al. 2004).

The most universally reported mechanisms of chemotherapy resistance are with elevated expression of FLIP and clAPs. At high expression levels, FLIP is a potent inhibitor of DISC-mediated apoptosis. Many cancer cells have highly active DR-signaling and targeted FLIP silencing alone can lead to DRindependent cell death in breast cancer cells (Day, Huang et al. 2008) and NSCLC cells (Chen, Liu et al. 2007; Wilson, Redmond et al. 2009).

Fas and FasL frequently contribute to apoptotic resistance in cancer. Different tumor types have been shown to express FasL, targeting immune cells for apoptosis thereby allowing them to escape immune surveillance (Walker, Saas et al. 1998). Pancreatic adenocarcinomas express FasL and are immune to Fas-mediated apoptosis by alterations in the Death Receptor signaling pathay (Ungefroren, Voss et al. 1998). Both SCLC and NSCLC cell lines have been 
shown to express FasL (Niehans, Brunner et al. 1997). Furthermore, acute leukemia and chronic myelogenous leukemia that express FasL have been shown to have a poorer prognosis (Lickliter, Kratzke et al. 1999). With the combination of loss of function mutations in proteins in the Fas pathway or upregulation of antiapoptotic proteins (CIAP, FLIP, Bcl-2) cancer cells become immune to autocrine effects of FasL.

TNF- $\alpha$ is an critical part of an effective immune system and is involved in inflammatory signaling. High levels of TNF- $\alpha$ secretion have been reported in numerous autoimmune disorders such as Crohn's Disease, rheumatoid arthritis, psoriasis, lupus and multiple sclerosis (Atzeni, Del Papa et al. 2004). It is also involved in the pathogenesis of diabetes, sepsis and cancer (Chen and Goeddel 2002). TNF- $\alpha$ is major component of proinflammatory signaling and is an indicator of both acute and chronic inflammation. The implications of inherent inflammatory signals are a major component in the tumor microenvironment. Acute high doses of TNF- $\alpha$ can aid the immune surveillance and cancer cell clearance (Watanabe, Niitsu et al. 1988; Lejeune, Ruegg et al. 1998). Chronic TNF- $\alpha$ exposure contributes to tumorigenic signaling through regulation of angiogenic factors, MMPs, cytokine and chemokine release (Balkwill and Mantovani 2001).

Although TNF- $\alpha$ can play a paradoxical role in tumor development, TNF- $\alpha$ antagonists and anti-inflammatory treatments in combination with cancer treatments are beneficial to halt tumorigenesis (Coussens and Werb 2002). TNF- $\alpha$ signaling antagonists such as Enbre ${ }^{\circledR}$, Infliximab ${ }^{\circledR}$, Adalimumab $^{\circledR}$ and 
Entanercept ${ }^{\circledR}$ have been widely successful in the management of numerous diseases with chronic TNF- $\alpha$ signaling (Heilig, Fiehn et al. 1993; Feldmann and Maini 2001). These are designed either as receptor antagonists or soluble receptor mimics and neutralize the effects of overabundant TNF- $\alpha$.

The association between chronic inflammation and cancer has long been established. NF-kB has been presented as the main component in this junction due to its transcriptional control over key inflammatory cytokines (Karin 2008). $\mathrm{NF}-\kappa \mathrm{B}$ is frequently constitutively expressed in cancer (Bharti and Aggarwal 2002). In addition to deregulation of the signaling pathways, mutations in NF-kB member proteins resulting in constitutive activation have also been reported and correlated with an incidence of cancer (Gilmore, Gapuzan et al. 2002).

Additionally, NF-kB has been shown to be essential for epithelial to mesenchymal transition, a hallmark in carcinoma development towards a metastatic tumor (Huber, Azoitei et al. 2004). Further research has implicated signaling to matrix metalloproteinases (MMPs) (Mehlen and Puisieux 2006), which are necessary for extracellular matrix degradation during invasion.

$\mathrm{NF}-\kappa \mathrm{B}$ activity can regulate and be regulated by ROS, however, the exact effects are controversial (Bubici, Papa et al. 2006). This is probably due to the fact that both ROS and NF-kB mediate so many different effects within the cell. Individually, the connection between ROS and cancer as well as the cohort of

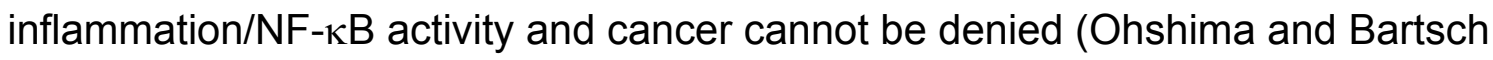
1994; Tamir and Tannenbaum 1996; Felley-Bosco 1998). 
In normal physiology the chronic signaling of TNF- $\alpha$ to NF-kB is terminated by the switch from prosurvival signaling complex (complex I) to the formation of a DISC (complex II). In aberrant signaling or unlimited cytokine provisions, the prosurvival signaling prevails leading to a constant positive feedback loop promoting inflammation. For example, IAPs which have been repeatedly reported to be upregulated in cancer (Vucic 2008) are induced by NF$\kappa \mathrm{B}$ activation and then further promote NF- $\mathrm{BB}$ activation through RIP-1 ubiquitination and the stabilization of complex 1 (Gyrd-Hansen and Meier). This abiding positive feedback loop would further promote the chronic inflammatory environment and tumorigenesis.

Alterations in protein signaling within the DR pathway of tumor cells are not the only oncogenic promoting force. The stroma surrounding a tumor has been shown to contribute to matrix remodeling, proliferation and angiogenesis (Condeelis and Pollard 2006). Tumor associated macrophages (TAMs), which play a significant role in DR-signaling, can provide an chronic inflammatory environment, rich in growth and angiogenic factors that support the growth of hyperproliferative cells (Coussens and Werb 2002). High levels of NO and large amounts of circulating TNF- $\alpha$ are frequently reported (Mantovani, Allavena et al. 2004). Additionally, fibroblasts can profoundly enhance carcinoma tumor formation through secreted growth factors such as transforming growth factor (TGF- $\beta$ ) and hepatocyte growth factor (HGF) (Bhowmick, Neilson et al. 2004). Additionally, abnormal or irradiated stroma can serve as a carcinogen to normal cells (Bissell and Radisky 2001). 


\section{Cell Migration}

Cellular migration is a complex signaling process that involves multiprotein organization and coordination. It is a vital component of embryogenesis, infection, inflammatory responses and wound healing (Horwitz and Webb 2003). In order to migrate, cells undergo molecular rearrangements to polarize (distinguishing between the front and rear of the cell) resulting in membrane extensions that abet movement (Lauffenburger and Horwitz 1996).

In the pathology of cancer, migration is one component of the metastatic cascade that results in advanced disease and poor prognosis (Weigelt, Peterse et al. 2005). Tumor cells must manipulate migratory processes for successful invasion and metastasis resulting in the establishment of tumor cells in distant organs. This is the major cause of death in most cancers (Inamura and Ishikawa; Weigelt, Peterse et al. 2005).

ROS are implicated in tumorigenesis and have been shown to contribute to migratory signaling. Long term oxidative stress is known to increase the invasive potential in mammary epithelial cells (Mori, Shibanuma et al. 2004). ROS has been reported to contribute to prolonged activation of promigratory signaling. ROS production has been shown to activate Erk1/2, JNK and NF-kB resulting in TGF- $\beta$-dependent astrocyte (Hsieh, Wang et al.) and glioblastoma 
(Chiu, Shen et al.) migration. ROS from the tumor microenvironment have also been shown to participate in tumor cell migration. Tobar et al. demonstrated that stromal mammary cells mediate MCF-7 breast cancer cells migration through NADPH oxidase 4 (Nox4) production of ROS (Tobar, Guerrero et al.).

Additionally Nox4-produced ROS has been shown to lead to migration in other cell types as well (Nam, Park et al.). With many cellular and tissue sources of ROS, these signaling intermediates could be key amplifiers for sustained signaling events involved in cell migration (see figure 1).

\section{Caveolin-1}

Caveolin-1 (Cav-1) is a multifunctional scaffolding protein that is the signature protein of a lipid raft subtype known as caveolae. These cellular membrane invaginations are especially prominent in endothelial cells, however, they are also formed in fibroblasts, adipocytes, smooth and skeletal muscle (Parton and Simons 2007). Caveolae are highly enriched in sphingolipids and cholesterol, a binding partner of Cav-1 (Parton, Hanzal-Bayer et al. 2006). Caveolae compartmentalize proteins and signaling pathways to initiate cell migration (Isshiki, Ando et al. 2002). Many proteins have been detected within caveolae including eNOS and VEGF receptor.

Cav-1 is part of the three protein Caveolin gene family. Caveolins are 21$24 \mathrm{kDa}$ membrane proteins with characterizing hairpin structure (Parton, Hanzal- 
Bayer et al. 2006). Cav-1 and Cav-2 are expressed in most cell types including epithelial, endothelial, mesenchymal and neuronal cells (Burgermeister, Liscovitch et al. 2008). Cav-3 is only found in muscle cells (Salanueva, Cerezo et al. 2007). Cav-1 has been reported to regulate many cellular processes involved in cancer including angiogenesis, migration, metastasis, proliferation and drug resistance (Goetz, Lajoie et al. 2008). Knockdown of Cav-1 in endothelial cells has been shown to inhibit cell polarization, migration and invasion (Galvez, Matias-Roman et al. 2004; Beardsley, Fang et al. 2005). Additionally, Cav-1 knockout MEFs are reported to have atypical morphology and migratory behavior due to changes in Rho GTPases and Src signaling (GrandeGarcia and del Pozo 2008).

In endothelial cells, caveolin is polarized within the cell during migration. Sun et.al. determined the 10 amino acid residues in the $\mathrm{N}$-terminus responsible for Cav-1 localization in the rear of the cell and rear polarization (Sun, Flynn et al. 2007). PTM of Cav-1 also helps determine its localization within the cell. Cav-1 is predominantly localized at the rear of a migrating cell. A subpopulation of Cav1 phosphorylated at tyrosine residue 14 are found at the leading edge and regulate focal adhesion (Beardsley, Fang et al. 2005). This Src-dependent phosphorylation mediates interleukin- 6 and insulin-like growth factor signals through PI3K and STAT3 (Podar and Anderson 2006). Additionally, VEGFmediated migration in multiple myeloma is Cav-1 dependent (Podar, Shringarpure et al. 2004). 
Cav-1 has both tumor suppressor and oncogenic activities and has been implicated in cancer progression and metastasis. Cav-1 contains a scaffolding domain (CSD) that binds proteins to exert tumor promoting or suppressive effects. For example, binding of NOS and cytosolic tyrosine kinases are inhibited through Cav-1 interaction whereas insulin receptor and adhesion proteins are kept active to support migration and angiogenesis (Williams and Lisanti 2005). The reported contradictory roles in migration could be dependent on cell specific differences in genotype and phenotype (Grande-Garcia and del Pozo 2008). Cav-1 inhibits migration in metastatic rat mammary adenocarcinoma (MTLn3) cells (Zhang, Razani et al. 2000).

Elevated expression of Cav-1 is highly correlated with poor prognosis in many types of cancer including gastrointestinal, esophageal, bladder, thyroid, brain, liver and renal cell (Burgermeister, Liscovitch et al. 2008). However, the tumorigenic role of Cav-1 may still vary by cell type. In prostate cancer, Cav-1 overexpression is associated with disease progression through interactions with Akt and angiogenic signaling (Thompson, Tahir et al.; Yang, Truong et al. 1999; Tahir, Yang et al. 2001). In pancreatic cancer, Cav-1 tissue expression positively correlates with tumor aggressiveness (Tanase 2008). Alternatively, Cav-1 overexpression in breast cancer cells inhibits anchorage-independent growth, matrix invasion and proliferation implicating Cav-1 expression as a tumor suppressor (Fiucci, Ravid et al. 2002). Additionally, it has been identified as a tumor suppressor in HER2/EGFR positive breast cancer (Park, Kim et al. 2005), mucoepidermoid carcinoma (Shi, Chen et al. 2007) and extrahepatic bile duct 
carcinoma (Murakami, Miyamoto et al. 2003). The conflicting reports of Cav-1 function also extend to drug sensitivity and treatment. It has been implicated in drug-resistant colon and breast cancer (Lavie, Fiucci et al. 1998; Yang, Truong et al. 1999) although also reported to restore chemosensitivity to cisplatin in oral cell carcinoma (Nakatani, Wada et al. 2005).

Cav-1 can clearly play a role in tumorigenesis. The variance of the contribution depends on the cell type and genetic signature of the cancer. Further mechanistic understanding of Cav-1 as both a tumor suppressor and promoter can potentially identify manageable targets within disease treatment and increase the patient prognosis. PTMs and redox can additionally modify Cav-1 signaling and should be investigated. NADPH oxidases have been identified in caveolae and lipid rafts (Zhang, Yi et al. 2006) indicating ROS are potentially prevalent near Cav-1. In Study 2 we examined the role of ROS on Cav-1 signaling in $\mathrm{H} 460$ lung adenocarcinoma cells to determine a pro- or antimigratory effect. We were particularly interested if different ROS species conferred a similar effect on Cav-1-dependent migration. 


\section{Impact and implications}

Currently, drug resistance and therapeutic selectivity are major issues in available effective cancer treatments. Understanding the differences between normal biology and cancer pathology will help determine potential targets for directed therapies. The signaling pathways that control apoptosis and cellular migration are important components in the progression of cancer. ROS and NO are noted to be key players in the advancement of pathology and disease not only as markers of toxicity but also by mediating specific signaling events (refer to figure 1). ROS, NO and DR-ligands are excessively produced in the tumor microenvironment and contribute to tumorigenesis (Maeda and Akaike 1998; Coussens and Werb 2002).

We are interested in how NO and ROS mediate protumorigenic effects such as developing mutations that are classified as the "Hallmarks of Cancer" (Hanahan and Weinberg 2000). Loss of tumor suppressors in combination with gain of function mutations can lead to apoptotic resistance, unlimited proliferation and migration and metastasis which are all seen in the advancement of cancer. Upregulation of antiapoptotic protein FLIP has been noted in multiple types of chemotherapy-resistant cancers. It has recently been associated with proliferative DR-pathways (Hyer, Samuel et al. 2006). The details of this association and the underlying mechanisms are not yet clear. In Study 1 we were interested in DR-mediated apoptosis and specifically examined how Snitrosylated FLIP, an antiapoptotic and proinflammatory protein, regulates 
downstream signaling. We show that S-nitrosylation of FLIP at residues 254 and 259 modulate potent transcription factor NF-kB through the redistribution of RIP1. We further show that S-nitrosylation of FLIP mediates protein processing into smaller protein fragments which could potentially alter signaling in pathways that classically recruit splice variant FLIPS.

Migration is a key component in tumor invasion and metastasis. A full understanding of the proteins mediating cell movement in the context of the tumor environment is necessary to effectively treat disease. In Study 2 we examined how ROS contributes to migration in NSCLC cells. We demonstrate that different ROS species mediate specific changes in migration and invasion in a Cav-1-dependent manner. We further show ROS-mediated Cav-1 expression reduces phosphorylated Akt. We additionally confirmed our results in melanoma cells.

Current literature indicates the importance of ROS and NO in the development and progression of cancer. Cancer cells and the supporting microenvironment are exposed to hypoxic and chronic inflammatory conditions that modulate ROS and NO production in vivo. These redox changes can dramatically alter cellular signaling and processes that control the fate of the cell. The functional implications of redox changes have only recently started to be dissected. Overall, this work will aim to decipher how ROS and NO can specifically alter known pathways in apoptosis and migration relevant in cancer progression. 


\section{References:}

Aggarwal, B. B. (2003). "Signalling pathways of the TNF superfamily: a doubleedged sword." Nat Rev Immunol 3(9): 745-56.

Aruoma, O. I., M. Grootveld, et al. (2006). "Free radicals in biology and medicine: from inflammation to biotechnology." Biofactors 27(1-4): 1-3.

Ashkenazi, A. and V. M. Dixit (1998). "Death receptors: signaling and modulation." Science 281(5381): 1305-8.

Ashkenazi, A., R. C. Pai, et al. (1999). "Safety and antitumor activity of recombinant soluble Apo2 ligand." J Clin Invest 104(2): 155-62.

Atzeni, F., N. Del Papa, et al. (2004). "CD69 expression on neutrophils from patients with rheumatoid arthritis." Clin Exp Rheumatol 22(3): 331-4.

Babior, B. M. (1978). "Oxygen-dependent microbial killing by phagocytes (first of two parts)." N Engl J Med 298(12): 659-68.

Bagneris, C., A. V. Ageichik, et al. (2008). "Crystal structure of a vFlip-IKKgamma complex: insights into viral activation of the IKK signalosome." Mol Cell 30(5): 620-31.

Balkwill, F. (2002). "Tumor necrosis factor or tumor promoting factor?" $\underline{\text { Cytokine }}$ Growth Factor Rev 13(2): 135-41.

Balkwill, F. and A. Mantovani (2001). "Inflammation and cancer: back to Virchow?" Lancet 357(9255): 539-45.

Barbouti, A., P. T. Doulias, et al. (2002). "DNA damage and apoptosis in hydrogen peroxide-exposed Jurkat cells: bolus addition versus continuous generation of $\mathrm{H}(2) \mathrm{O}(2)$." Free Radic Biol Med 33(5): 691-702.

Beardsley, A., K. Fang, et al. (2005). "Loss of caveolin-1 polarity impedes endothelial cell polarization and directional movement." $\underline{\mathrm{J} \text { Biol Chem }}$ 280(5): 3541-7. 
Benhar, M., M. T. Forrester, et al. (2009). "Protein denitrosylation: enzymatic mechanisms and cellular functions." Nat Rev Mol Cell Biol 10(10): 721-32.

Bharti, A. C. and B. B. Aggarwal (2002). "Nuclear factor-kappa B and cancer: its role in prevention and therapy." Biochem Pharmacol 64(5-6): 883-8.

Bhowmick, N. A., E. G. Neilson, et al. (2004). "Stromal fibroblasts in cancer initiation and progression." Nature 432(7015): 332-7.

Bissell, M. J. and D. Radisky (2001). "Putting tumours in context." Nat Rev Cancer 1(1): 46-54.

Bredt, D. S. (2003). "Nitric oxide signaling specificity--the heart of the problem." $\underline{J}$ Cell Sci 116(Pt 1): 9-15.

Bubici, C., S. Papa, et al. (2006). "Mutual cross-talk between reactive oxygen species and nuclear factor-kappa B: molecular basis and biological significance." Oncogene 25(51): 6731-48.

Budd, R. C. (2002). "Death receptors couple to both cell proliferation and apoptosis." J Clin Invest 109(4): 437-41.

Burdon, R. H. (1995). "Superoxide and hydrogen peroxide in relation to mammalian cell proliferation." Free Radic Biol Med 18(4): 775-94.

Burgermeister, E., M. Liscovitch, et al. (2008). "Caveats of caveolin-1 in cancer progression." Cancer Lett 268(2): 187-201.

Carew, J. S. and P. Huang (2002). "Mitochondrial defects in cancer." Mol Cancer 1: 9 .

Casteilla, L., M. Rigoulet, et al. (2001). "Mitochondrial ROS metabolism: modulation by uncoupling proteins." IUBMB Life 52(3-5): 181-8.

Chang, D. W., Z. Xing, et al. (2003). "Interdimer processing mechanism of procaspase-8 activation." EMBO J 22(16): 4132-42. 
Chanvorachote, P., U. Nimmannit, et al. (2005). "Nitric oxide negatively regulates Fas CD95-induced apoptosis through inhibition of ubiquitin-proteasomemediated degradation of FLICE inhibitory protein." J Biol Chem 280(51): 42044-50.

Chawla-Sarkar, M., S. I. Bae, et al. (2004). "Downregulation of Bcl-2, FLIP or IAPs (XIAP and survivin) by siRNAs sensitizes resistant melanoma cells to Apo2L/TRAIL-induced apoptosis." Cell Death Differ 11(8): 915-23.

Chen, G. and D. V. Goeddel (2002). "TNF-R1 signaling: a beautiful pathway." Science 296(5573): 1634-5.

Chen, S., X. Liu, et al. (2007). "CCAAT/enhancer binding protein homologous protein-dependent death receptor 5 induction and ubiquitin/proteasomemediated cellular FLICE-inhibitory protein down-regulation contribute to enhancement of tumor necrosis factor-related apoptosis-inducing ligandinduced apoptosis by dimethyl-celecoxib in human non small-cell lung cancer cells." Mol Pharmacol 72(5): 1269-79.

Chen, X., J. J. Subleski, et al. (2008). "Cutting edge: expression of TNFR2 defines a maximally suppressive subset of mouse CD4+CD25+FoxP3+ T regulatory cells: applicability to tumor-infiltrating T regulatory cells." $\underline{\mathrm{J}}$ Immunol 180(10): 6467-71.

Chinery, R., J. A. Brockman, et al. (1997). "Antioxidants enhance the cytotoxicity of chemotherapeutic agents in colorectal cancer: a p53-independent induction of p21WAF1/CIP1 via C/EBPbeta." Nat Med 3(11): 1233-41.

Chinnaiyan, A. M., K. O'Rourke, et al. (1995). "FADD, a novel death domaincontaining protein, interacts with the death domain of Fas and initiates apoptosis." Cell 81(4): 505-12.

Chipuk, J. E., L. Bouchier-Hayes, et al. (2006). "Mitochondrial outer membrane permeabilization during apoptosis: the innocent bystander scenario." Death Differ 13(8): 1396-402.

Chiu, W. T., S. C. Shen, et al. "Contribution of reactive oxygen species to migration/invasion of human glioblastoma cells U87 via ERK-dependent COX-2/PGE(2) activation." Neurobiol Dis 37(1): 118-29. 
Chun, H. J., L. Zheng, et al. (2002). "Pleiotropic defects in lymphocyte activation caused by caspase-8 mutations lead to human immunodeficiency." Nature 419(6905): 395-9.

Chuntharapai, A., K. Dodge, et al. (2001). "Isotype-dependent inhibition of tumor growth in vivo by monoclonal antibodies to death receptor $4 . " \mathrm{~J} \mathrm{Immunol}$ 166(8): 4891-8.

Condeelis, J. and J. W. Pollard (2006). "Macrophages: obligate partners for tumor cell migration, invasion, and metastasis." Cell 124(2): 263-6.

Coussens, L. M. and Z. Werb (2002). "Inflammation and cancer." Nature 420(6917): 860-7.

Crowell, J. A., V. E. Steele, et al. (2003). "Is inducible nitric oxide synthase a target for chemoprevention?" Mol Cancer Ther 2(8): 815-23.

D'Autreaux, B. and M. B. Toledano (2007). "ROS as signalling molecules: mechanisms that generate specificity in ROS homeostasis." Nat Rev Mol Cell Biol 8(10): 813-24.

Day, T. W., S. Huang, et al. (2008). "c-FLIP knockdown induces ligandindependent DR5-, FADD-, caspase-8-, and caspase-9-dependent apoptosis in breast cancer cells." Biochem Pharmacol 76(12): 1694-704.

Declercq, W., T. Vanden Berghe, et al. (2009). "RIP kinases at the crossroads of cell death and survival." Cell 138(2): 229-32.

Devin, A., A. Cook, et al. (2000). "The distinct roles of TRAF2 and RIP in IKK activation by TNF-R1: TRAF2 recruits IKK to TNF-R1 while RIP mediates IKK activation." Immunity 12(4): 419-29.

Dhar, A., M. R. Young, et al. (2002). "The role of AP-1, NF-kappaB and ROS/NOS in skin carcinogenesis: the JB6 model is predictive." Mol Cell Biochem 234-235(1-2): 185-93.

Dohrman, A., T. Kataoka, et al. (2005). "Cellular FLIP (long form) regulates CD8+ T cell activation through caspase-8-dependent NF-kappa B activation." $\underline{\mathrm{J}}$ Immunol 174(9): 5270-8. 
Edwards, P., J. C. Cendan, et al. (1996). "Tumor cell nitric oxide inhibits cell growth in vitro, but stimulates tumorigenesis and experimental lung metastasis in vivo." J Surg Res 63(1): 49-52.

Ehrhardt, H., S. Fulda, et al. (2003). "TRAIL induced survival and proliferation in cancer cells resistant towards TRAIL-induced apoptosis mediated by NFkappaB." Oncogene 22(25): 3842-52.

Emery, J. G., P. McDonnell, et al. (1998). "Osteoprotegerin is a receptor for the

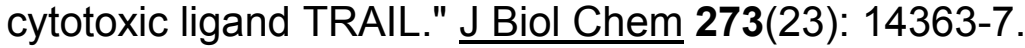

Falschlehner, C., T. M. Ganten, et al. (2009). "TRAIL and other TRAIL receptor agonists as novel cancer therapeutics." Adv Exp Med Biol 647: 195-206.

Feldmann, M. and R. N. Maini (2001). "Anti-TNF alpha therapy of rheumatoid arthritis: what have we learned?" Annu Rev Immunol 19: 163-96.

Felley-Bosco, E. (1998). "Role of nitric oxide in genotoxicity: implication for carcinogenesis." Cancer Metastasis Rev 17(1): 25-37.

Festjens, N., T. Vanden Berghe, et al. (2007). "RIP1, a kinase on the crossroads of a cell's decision to live or die." Cell Death Differ 14(3): 400-10.

Finocchietto, P. V., M. C. Franco, et al. (2009). "Mitochondrial nitric oxide synthase: a masterpiece of metabolic adaptation, cell growth, transformation, and death." Exp Biol Med (Maywood) 234(9): 1020-8.

Fisher, G. H., F. J. Rosenberg, et al. (1995). "Dominant interfering Fas gene mutations impair apoptosis in a human autoimmune lymphoproliferative syndrome." Cell 81(6): 935-46.

Fiucci, G., D. Ravid, et al. (2002). "Caveolin-1 inhibits anchorage-independent growth, anoikis and invasiveness in MCF-7 human breast cancer cells." Oncogene 21(15): 2365-75.

Fotin-Mleczek, M., F. Henkler, et al. (2002). "Apoptotic crosstalk of TNF receptors: TNF-R2-induces depletion of TRAF2 and IAP proteins and accelerates TNF-R1-dependent activation of caspase-8." J Cell Sci 115(Pt 13): 2757-70. 
Fukumura, D., S. Kashiwagi, et al. (2006). "The role of nitric oxide in tumour progression." Nat Rev Cancer 6(7): 521-34.

Galaris, D., V. Skiada, et al. (2008). "Redox signaling and cancer: the role of "labile" iron." Cancer Lett 266(1): 21-9.

Galvez, B. G., S. Matias-Roman, et al. (2004). "Caveolae are a novel pathway for membrane-type 1 matrix metalloproteinase traffic in human endothelial cells." Mol Biol Cell 15(2): 678-87.

Gazitt, Y. (1999). "TRAIL is a potent inducer of apoptosis in myeloma cells derived from multiple myeloma patients and is not cytotoxic to hematopoietic stem cells." Leukemia 13(11): 1817-24.

Gilmore, T., M. E. Gapuzan, et al. (2002). "Rel/NF-kappa B/l kappa B signal transduction in the generation and treatment of human cancer." Cancer Lett 181(1): 1-9.

Gius, D. and D. R. Spitz (2006). "Redox signaling in cancer biology." Antioxid Redox Signal 8(7-8): 1249-52.

Goetz, J. G., P. Lajoie, et al. (2008). "Caveolin-1 in tumor progression: the good, the bad and the ugly." Cancer Metastasis Rev 27(4): 715-35.

Goldstein, S., D. Meyerstein, et al. (1993). "The Fenton reagents." Free Radic Biol Med 15(4): 435-45.

Golks, A., D. Brenner, et al. (2006). "The c-FLIP-NH2 terminus (p22-FLIP) induces NF-kappaB activation." J Exp Med 203(5): 1295-305.

Grande-Garcia, A. and M. A. del Pozo (2008). "Caveolin-1 in cell polarization and directional migration." Eur J Cell Biol 87(8-9): 641-7.

Green, D. R. and G. P. Amarante-Mendes (1998). "The point of no return: mitochondria, caspases, and the commitment to cell death." Results Probl Cell Differ 24: 45-61. 
Gyrd-Hansen, M. and P. Meier "IAPs: from caspase inhibitors to modulators of NF-kappaB, inflammation and cancer." Nat Rev Cancer 10(8): 561-74.

Hahne, M., D. Rimoldi, et al. (1996). "Melanoma cell expression of Fas(Apo1/CD95) ligand: implications for tumor immune escape." $\underline{\text { Science }}$ 274(5291): 1363-6.

Hanahan, D. and R. A. Weinberg (2000). "The hallmarks of cancer." Cell 100(1): 57-70.

Hancock, J. T. (2009). "The role of redox in signal transduction." Methods Mol Biol 476: 1-9.

Harada, K., Supriatno, et al. (2004). "Overexpression of iNOS gene suppresses the tumorigenicity and metastasis of oral cancer cells." In Vivo 18(4): 44955 .

He, J. Q., S. K. Saha, et al. (2007). "Specificity of TRAF3 in its negative regulation of the noncanonical NF-kappa B pathway." J Biol Chem 282(6): 3688-94.

Heilig, B., C. Fiehn, et al. (1993). "Evaluation of soluble tumor necrosis factor (TNF) receptors and TNF receptor antibodies in patients with systemic lupus erythematodes, progressive systemic sclerosis, and mixed connective tissue disease." J Clin Immunol 13(5): 321-8.

Horwitz, R. and D. Webb (2003). "Cell migration." Curr Biol 13(19): R756-9.

Houston, A. and J. O'Connell (2004). "The Fas signalling pathway and its role in the pathogenesis of cancer." Curr Opin Pharmacol 4(4): 321-6.

Hsieh, H. L., H. H. Wang, et al. "Transforming growth factor-beta1 induces matrix metalloproteinase- 9 and cell migration in astrocytes: roles of ROSdependent ERK- and JNK-NF-kappaB pathways." J Neuroinflammation 7: 88.

Huber, M. A., N. Azoitei, et al. (2004). "NF-kappaB is essential for epithelialmesenchymal transition and metastasis in a model of breast cancer progression." J Clin Invest 114(4): 569-81. 
Hussain, S. P., P. He, et al. (2008). "Nitric oxide is a key component in inflammation-accelerated tumorigenesis." Cancer Res 68(17): 7130-6.

Hyer, M. L., T. Samuel, et al. (2006). "The FLIP-side of Fas signaling." Clin Cancer Res 12(20 Pt 1): 5929-31.

Ichikawa, K., W. Liu, et al. (2001). "Tumoricidal activity of a novel anti-human DR5 monoclonal antibody without hepatocyte cytotoxicity." Nat Med 7(8): 954-60.

Ignarro, L. J. (2000). Nitric Oxide: Biology and Pathobiology. Orlando, FL, Academic Press, Science.

Inamura, K. and Y. Ishikawa "Lung cancer progression and metastasis from the prognostic point of view." Clin Exp Metastasis 27(6): 389-97.

Irmler, M., M. Thome, et al. (1997). "Inhibition of death receptor signals by cellular FLIP." Nature 388(6638): 190-5.

Isshiki, M., J. Ando, et al. (2002). "Sites of $\mathrm{Ca}(2+)$ wave initiation move with caveolae to the trailing edge of migrating cells." J Cell Sci 115(Pt 3): 47584.

Jackson-Bernitsas, D. G., H. Ichikawa, et al. (2007). "Evidence that TNF-TNFR1TRADD-TRAF2-RIP-TAK1-IKK pathway mediates constitutive NF-kappaB activation and proliferation in human head and neck squamous cell carcinoma." Oncogene 26(10): 1385-97.

Jaffrey, S. R. and S. H. Snyder (2001). "The biotin switch method for the detection of S-nitrosylated proteins." Sci STKE 2001(86): pl1.

Jenkins, D. C., I. G. Charles, et al. (1995). "Roles of nitric oxide in tumor growth." Proc Natl Acad Sci U S A 92(10): 4392-6.

Jha, M. N., J. S. Bedford, et al. (1999). "Vitamin E (d-alpha-tocopheryl succinate) decreases mitotic accumulation in gamma-irradiated human tumor, but not in normal, cells." Nutr Cancer 35(2): 189-94. 
Johnson, T. M., Z. X. Yu, et al. (1996). "Reactive oxygen species are downstream mediators of p53-dependent apoptosis." Proc Natl Acad Sci U S A 93(21): 11848-52.

Ju, S. T., D. J. Panka, et al. (1995). "Fas(CD95)/FasL interactions required for programmed cell death after T-cell activation." Nature 373(6513): 444-8.

Kamata, H. and H. Hirata (1999). "Redox regulation of cellular signalling." Cell Signal 11(1): 1-14.

Karin, M. (2008). "The IkappaB kinase - a bridge between inflammation and cancer." Cell Res 18(3): 334-42.

Kataoka, T., R. C. Budd, et al. (2000). "The caspase-8 inhibitor FLIP promotes activation of NF-kappaB and Erk signaling pathways." Curr Biol 10(11): 640-8.

Kataoka, T. and J. Tschopp (2004). "N-terminal fragment of c-FLIP(L) processed by caspase 8 specifically interacts with TRAF2 and induces activation of the NF-kappaB signaling pathway." Mol Cell Biol 24(7): 2627-36.

Kawanishi, S., Y. Hiraku, et al. (2006). "Oxidative and nitrative DNA damage in animals and patients with inflammatory diseases in relation to inflammation-related carcinogenesis." Biol Chem 387(4): 365-72.

Keane, M. M., S. A. Ettenberg, et al. (1999). "Chemotherapy augments TRAILinduced apoptosis in breast cell lines." Cancer Res 59(3): 734-41.

Kelliher, M. A., S. Grimm, et al. (1998). "The death domain kinase RIP mediates the TNF-induced NF-kappaB signal." Immunity 8(3): 297-303.

Kennedy, N. J., T. Kataoka, et al. (1999). "Caspase activation is required for T cell proliferation." J Exp Med 190(12): 1891-6.

Kerr, J. F., A. H. Wyllie, et al. (1972). "Apoptosis: a basic biological phenomenon with wide-ranging implications in tissue kinetics." Br J Cancer 26(4): 23957. 
Kim, B. M. and H. W. Chung (2007). "Hypoxia/reoxygenation induces apoptosis through a ROS-mediated caspase-8/Bid/Bax pathway in human lymphocytes." Biochem Biophys Res Commun 363(3): 745-50.

Kim, K., M. J. Fisher, et al. (2000). "Molecular determinants of response to TRAIL in killing of normal and cancer cells." Clin Cancer Res 6(2): 335-46.

Kim, S. F., D. A. Huri, et al. (2005). "Inducible nitric oxide synthase binds, Snitrosylates, and activates cyclooxygenase-2." Science 310(5756): 196670.

Krammer, P. H. (2000). "CD95's deadly mission in the immune system." Nature 407(6805): 789-95.

Labriola, D. and R. Livingston (1999). "Possible interactions between dietary antioxidants and chemotherapy." Oncology (Williston Park) 13(7): 1003-8; discussion 1008, 1011-2.

Lambeth, J. D. (2007). "Nox enzymes, ROS, and chronic disease: an example of antagonistic pleiotropy." Free Radic Biol Med 43(3): 332-47.

Lamkanfi, M., N. Festjens, et al. (2007). "Caspases in cell survival, proliferation and differentiation." Cell Death Differ 14(1): 44-55.

Lander, H. M. (1997). "An essential role for free radicals and derived species in signal transduction." FASEB J 11(2): 118-24.

Lauffenburger, D. A. and A. F. Horwitz (1996). "Cell migration: a physically integrated molecular process." Cell 84(3): 359-69.

Lavie, Y., G. Fiucci, et al. (1998). "Up-regulation of caveolae and caveolar constituents in multidrug-resistant cancer cells." J Biol Chem 273(49): 32380-3.

Lavrik, I., A. Golks, et al. (2005). "Death receptor signaling." J Cell Sci 118(Pt 2): 265-7. 
Lejeune, F. J., C. Ruegg, et al. (1998). "Clinical applications of TNF-alpha in cancer." Curr Opin Immunol 10(5): 573-80.

$\mathrm{Li}, \mathrm{J}$. and J. Yuan (2008). "Caspases in apoptosis and beyond." Oncogene 27(48): 6194-206.

Lickliter, J. D., R. A. Kratzke, et al. (1999). "Fas ligand is highly expressed in acute leukemia and during the transformation of chronic myeloid leukemia to blast crisis." Exp Hematol 27(10): 1519-27.

Ling, Y. H., L. Liebes, et al. (2003). "Reactive oxygen species generation and mitochondrial dysfunction in the apoptotic response to Bortezomib, a novel proteasome inhibitor, in human H460 non-small cell lung cancer cells." $\underline{\mathrm{J}}$ Biol Chem 278(36): 33714-23.

Lowenstein, C. J. and E. Padalko (2004). "iNOS (NOS2) at a glance." J Cell Sci 117(Pt 14): 2865-7.

Maccarrone, M. and B. Brune (2009). "Redox regulation in acute and chronic inflammation." Cell Death Differ 16(8): 1184-6.

Maeda, H. and T. Akaike (1998). "Nitric oxide and oxygen radicals in infection, inflammation, and cancer." Biochemistry (Mosc) 63(7): 854-65.

Mannick, J. B., C. Schonhoff, et al. (2001). "S-Nitrosylation of mitochondrial caspases." J Cell Biol 154(6): 1111-6.

Mantovani, A., P. Allavena, et al. (2004). "Tumour-associated macrophages as a prototypic type II polarised phagocyte population: role in tumour progression." Eur J Cancer 40(11): 1660-7.

Marshall, H. E., D. T. Hess, et al. (2004). "S-nitrosylation: physiological regulation of NF-kappaB." Proc Natl Acad Sci U S A 101(24): 8841-2.

Marshall, H. E. and J. S. Stamler (2001). "Inhibition of NF-kappa B by Snitrosylation." Biochemistry 40(6): 1688-93. 
Mathew, R. and E. White (2006). "FLIPping the balance between apoptosis and proliferation in thyroid cancer." Clin Cancer Res 12(12): 3648-51.

Mehlen, P. and A. Puisieux (2006). "Metastasis: a question of life or death." Nat Rev Cancer 6(6): 449-58.

Micheau, O., M. Thome, et al. (2002). "The long form of FLIP is an activator of caspase-8 at the Fas death-inducing signaling complex." $\mathrm{J}$ Biol Chem 277(47): 45162-71.

Micheau, O. and J. Tschopp (2003). "Induction of TNF receptor I-mediated apoptosis via two sequential signaling complexes." Cell 114(2): 181-90.

Mitchell, D. A. and M. A. Marletta (2005). "Thioredoxin catalyzes the S-nitrosation of the caspase-3 active site cysteine." Nat Chem Biol 1(3): 154-8.

Mori, K., M. Shibanuma, et al. (2004). "Invasive potential induced under longterm oxidative stress in mammary epithelial cells." Cancer Res 64(20): 7464-72.

Muppidi, J. R. and R. M. Siegel (2004). "Ligand-independent redistribution of Fas (CD95) into lipid rafts mediates clonotypic T cell death." Nat Immunol 5(2): 182-9.

Murakami, S., M. Miyamoto, et al. (2003). "Caveolin-I overexpression is a favourable prognostic factor for patients with extrahepatic bile duct carcinoma." Br J Cancer 88(8): 1234-8.

Nakatani, K., T. Wada, et al. (2005). "Expression of caveolin-1 and its correlation with cisplatin sensitivity in oral squamous cell carcinoma." $\mathrm{J}$ Cancer Res Clin Oncol 131(7): 445-52.

Nam, H. J., Y. Y. Park, et al. "Co-treatment with hepatocyte growth factor and TGF-beta1 enhances migration of HaCaT cells through NADPH oxidasedependent ROS generation." Exp Mol Med 42(4): 270-9.

Nastiuk, K. L. and J. J. Krolewski (2008). "FLIP-ping out: death receptor signaling in the prostate." Cancer Biol Ther 7(8): 1171-9. 
Natoli, G. and L. M. Austenaa (2008). "A birthday gift for TRADD." Nat Immunol 9(9): 1015-6.

Niehans, G. A., T. Brunner, et al. (1997). "Human lung carcinomas express Fas ligand." Cancer Res 57(6): 1007-12.

Nussler, A. K., M. Di Silvio, et al. (1992). "Stimulation of the nitric oxide synthase pathway in human hepatocytes by cytokines and endotoxin." $\underline{\mathrm{J} \text { Exp Med }}$ 176(1): 261-4.

Ohshima, H. and H. Bartsch (1994). "Chronic infections and inflammatory processes as cancer risk factors: possible role of nitric oxide in carcinogenesis." Mutat Res 305(2): 253-64.

Oikonomou, E., V. Kosmidou, et al. (2009). "TRAIL receptor upregulation and the implication of KRAS/BRAF mutations in human colon cancer tumors." Int J Cancer 125(9): 2127-35.

Osborn, S. L., S. J. Sohn, et al. (2007). "Constitutive phosphorylation mutation in Fas-associated death domain (FADD) results in early cell cycle defects." $\underline{\mathrm{J}}$ Biol Chem 282(31): 22786-92.

Paige, J. S., G. Xu, et al. (2008). "Nitrosothiol reactivity profiling identifies Snitrosylated proteins with unexpected stability." Chem Biol 15(12): 130716.

Park, S. S., J. E. Kim, et al. (2005). "Caveolin-1 is down-regulated and inversely correlated with HER2 and EGFR expression status in invasive ductal carcinoma of the breast." Histopathology 47(6): 625-30.

Parton, R. G., M. Hanzal-Bayer, et al. (2006). "Biogenesis of caveolae: a structural model for caveolin-induced domain formation." J Cell Sci 119(Pt 5): 787-96.

Parton, R. G. and K. Simons (2007). "The multiple faces of caveolae." Nat Rev Mol Cell Biol 8(3): 185-94.

Patel, R. P., J. McAndrew, et al. (1999). "Biological aspects of reactive nitrogen species." Biochim Biophys Acta 1411(2-3): 385-400. 
Pelicano, H., D. Carney, et al. (2004). "ROS stress in cancer cells and therapeutic implications." Drug Resist Updat 7(2): 97-110.

Pelicano, H., R. H. Xu, et al. (2006). "Mitochondrial respiration defects in cancer cells cause activation of Akt survival pathway through a redox-mediated mechanism." J Cell Biol 175(6): 913-23.

Perkins, N. D. (2007). "Integrating cell-signalling pathways with NF-kappaB and IKK function." Nat Rev Mol Cell Biol 8(1): 49-62.

Pitti, R. M., S. A. Marsters, et al. (1996). "Induction of apoptosis by Apo-2 ligand, a new member of the tumor necrosis factor cytokine family." J Biol Chem 271(22): 12687-90.

Podar, K. and K. C. Anderson (2006). "Caveolin-1 as a potential new therapeutic target in multiple myeloma." Cancer Lett 233(1): 10-5.

Podar, K., R. Shringarpure, et al. (2004). "Caveolin-1 is required for vascular endothelial growth factor-triggered multiple myeloma cell migration and is targeted by bortezomib." Cancer Res 64(20): 7500-6.

Poyet, J. L., S. M. Srinivasula, et al. (2000). "Activation of the Ikappa B kinases by RIP via IKKgamma /NEMO-mediated oligomerization." $\underline{\mathrm{J} \text { Biol Chem }}$ 275(48): 37966-77.

Rieger, J., U. Naumann, et al. (1998). "APO2 ligand: a novel lethal weapon against malignant glioma?" FEBS Lett 427(1): 124-8.

Salanueva, I. J., A. Cerezo, et al. (2007). "Integrin regulation of caveolin function." J Cell Mol Med 11(5): 969-80.

Salganik, R. I. (2001). "The benefits and hazards of antioxidants: controlling apoptosis and other protective mechanisms in cancer patients and the

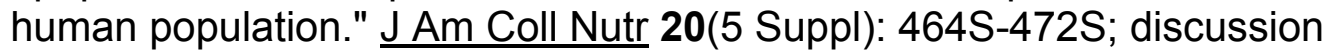
473S-475S.

Sallmyr, A., J. Fan, et al. (2008). "Genomic instability in myeloid malignancies: increased reactive oxygen species (ROS), DNA double strand breaks (DSBs) and error-prone repair." Cancer Lett 270(1): 1-9. 
Scaffidi, C., S. Fulda, et al. (1998). "Two CD95 (APO-1/Fas) signaling pathways." EMBO J 17(6): 1675-87.

Scaffidi, C., I. Schmitz, et al. (1999). "The role of c-FLIP in modulation of CD95induced apoptosis." J Biol Chem 274(3): 1541-8.

Schneider, P., M. Thome, et al. (1997). "TRAIL receptors 1 (DR4) and 2 (DR5) signal FADD-dependent apoptosis and activate NF-kappaB." Immunity 7(6): 831-6.

Schurmann, P. and J. P. Jacquot (2000). "Plant Thioredoxin Systems Revisited." Annu Rev Plant Physiol Plant Mol Biol 51: 371-400.

Secchiero, P., D. Milani, et al. (2003). "Tumor necrosis factor (TNF)-related apoptosis-inducing ligand (TRAIL) and TNF-alpha promote the NFkappaB-dependent maturation of normal and leukemic myeloid cells." $\underline{J}$ Leukoc Biol 74(2): 223-32.

Seifried, H. E., S. S. McDonald, et al. (2003). "The antioxidant conundrum in cancer." Cancer Res 63(15): 4295-8.

Sheridan, J. P., S. A. Marsters, et al. (1997). "Control of TRAIL-induced apoptosis by a family of signaling and decoy receptors." Science 277(5327): 818-21.

Shi, L., X. M. Chen, et al. (2007). "Expression of caveolin-1 in mucoepidermoid carcinoma of the salivary glands: correlation with vascular endothelial growth factor, microvessel density, and clinical outcome." Cancer 109(8): 1523-31.

Shikama, Y., M. Yamada, et al. (2003). "Caspase-8 and caspase-10 activate NFkappaB through RIP, NIK and IKKalpha kinases." Eur J Immunol 33(7): 1998-2006.

Shin, M. S., H. S. Kim, et al. (2001). "Mutations of tumor necrosis factor-related apoptosis-inducing ligand receptor 1 (TRAIL-R1) and receptor 2 (TRAILR2) genes in metastatic breast cancers." Cancer Res 61(13): 4942-6. 
Siedlar, M., B. Mytar, et al. (1999). "Demonstration of iNOS-mRNA and iNOS in human monocytes stimulated with cancer cells in vitro." 65(5): 597-604.

Silke, J. and R. Brink "Regulation of TNFRSF and innate immune signalling complexes by TRAFs and clAPs." Cell Death Differ 17(1): 35-45.

Simon, H. U., A. Haj-Yehia, et al. (2000). "Role of reactive oxygen species (ROS) in apoptosis induction." Apoptosis 5(5): 415-8.

Stewart, T. J. and S. I. Abrams (2008). "How tumours escape mass destruction." Oncogene 27(45): 5894-903.

Sun, X. H., D. C. Flynn, et al. (2007). "Identification of a novel domain at the $\mathrm{N}$ terminus of caveolin-1 that controls rear polarization of the protein and caveolae formation." J Biol Chem 282(10): 7232-41.

Suzuki, Y. J., H. J. Forman, et al. (1997). "Oxidants as stimulators of signal transduction." Free Radic Biol Med 22(1-2): 269-85.

Tada, K., T. Okazaki, et al. (2001). "Critical roles of TRAF2 and TRAF5 in tumor necrosis factor-induced NF-kappa B activation and protection from cell death." J Biol Chem 276(39): 36530-4.

Tahir, S. A., G. Yang, et al. (2001). "Secreted caveolin-1 stimulates cell survival/clonal growth and contributes to metastasis in androgeninsensitive prostate cancer." Cancer Res 61(10): 3882-5.

Takahashi, M., T. Kitahashi, et al. (2008). "Increased expression of inducible nitric oxide synthase (iNOS) in N-nitrosobis(2-oxopropyl)amine-induced hamster pancreatic carcinogenesis and prevention of cancer development by ONO-1714, an iNOS inhibitor." Carcinogenesis 29(8): 1608-13.

Tamir, S. and S. R. Tannenbaum (1996). "The role of nitric oxide (NO.) in the carcinogenic process." Biochim Biophys Acta 1288(2): F31-6.

Tanase, C. P. (2008). "Caveolin-1: a marker for pancreatic cancer diagnosis." Expert Rev Mol Diagn 8(4): 395-404. 
Tang, W., W. Wang, et al. (2009). "Tumour necrosis factor-related apoptosisinducing ligand (TRAIL)-induced chemokine release in both TRAILresistant and TRAIL-sensitive cells via nuclear factor kappa B." $\underline{\text { FEBS J }}$ 276(2): 581-93.

Tannenbaum, S. R. and F. M. White (2006). "Regulation and specificity of Snitrosylation and denitrosylation." ACS Chem Biol 1(10): 615-8.

Tartaglia, L. A., T. M. Ayres, et al. (1993). "A novel domain within the $55 \mathrm{kd}$ TNF receptor signals cell death." Cell 74(5): 845-53.

Thome, M., P. Schneider, et al. (1997). "Viral FLICE-inhibitory proteins (FLIPs) prevent apoptosis induced by death receptors." Nature 386(6624): 517-21.

Thompson, T. C., S. A. Tahir, et al. "The role of caveolin-1 in prostate cancer: clinical implications." Prostate Cancer Prostatic Dis 13(1): 6-11.

Thomsen, L. L., J. M. Scott, et al. (1997). "Selective inhibition of inducible nitric oxide synthase inhibits tumor growth in vivo: studies with $1400 \mathrm{~W}$, a novel inhibitor." Cancer Res 57(15): 3300-4.

Tobar, N., J. Guerrero, et al. "NOX4-dependent ROS production by stromal mammary cells modulates epithelial MCF-7 cell migration." $\mathrm{Br} \mathrm{J}$ Cancer 103(7): 1040-7.

Toyokuni, S. (2004). "Redox control of carcinogenesis and tumor biology." Antioxid Redox Signal 6(3): 481-2.

Trauth, B. C., C. Klas, et al. (1989). "Monoclonal antibody-mediated tumor regression by induction of apoptosis." Science 245(4915): 301-5.

Trauzold, A., C. Roder, et al. (2005). "CD95 and TRAF2 promote invasiveness of pancreatic cancer cells." FASEB J 19(6): 620-2.

Ungefroren, H., M. Voss, et al. (1998). "Human pancreatic adenocarcinomas express Fas and Fas ligand yet are resistant to Fas-mediated apoptosis." Cancer Res 58(8): 1741-9. 
Ushio-Fukai, M. (2006). "Localizing NADPH oxidase-derived ROS." Sci STKE 2006(349): re8.

van Galen, J. C., J. J. Muris, et al. (2008). "Expression of TNF-receptor associated factor 2 correlates with poor progression-free survival time in ABC-like primary nodal diffuse large B-cell lymphomas." Histopathology 52(5): 578-84.

Varfolomeev, E., T. Goncharov, et al. (2008). "c-IAP1 and c-IAP2 are critical mediators of tumor necrosis factor alpha (TNFalpha)-induced NF-kappaB activation." J Biol Chem 283(36): 24295-9.

Via, C. S., P. Nguyen, et al. (1996). "A major role for the Fas pathway in acute graft-versus-host disease." $\mathrm{J}$ Immunol 157(12): 5387-93.

Voss, M., M. Lettau, et al. (2008). "Posttranslational regulation of Fas ligand function." Cell Commun Signal 6: 11.

Vucic, D. (2008). "Targeting IAP (inhibitor of apoptosis) proteins for therapeutic intervention in tumors." Curr Cancer Drug Targets 8(2): 110-7.

Wajant, H. (2002). "The Fas signaling pathway: more than a paradigm." Science 296(5573): 1635-6.

Walker, P. R., P. Saas, et al. (1998). "Tumor expression of Fas ligand (CD95L) and the consequences." Curr Opin Immunol 10(5): 564-72.

Wang, S. (2008). "The promise of cancer therapeutics targeting the TNF-related apoptosis-inducing ligand and TRAIL receptor pathway." Oncogene 27(48): 6207-15.

Watanabe, N., Y. Niitsu, et al. (1988). "Synergistic cytotoxic and antitumor effects of recombinant human tumor necrosis factor and hyperthermia." Cancer Res 48(3): 650-3.

Weigelt, B., J. L. Peterse, et al. (2005). "Breast cancer metastasis: markers and models." Nat Rev Cancer 5(8): 591-602. 
Werner, A. B., E. de Vries, et al. (2002). "TRAIL receptor and CD95 signal to mitochondria via FADD, caspase-8/10, Bid, and Bax but differentially regulate events downstream from truncated Bid." J Biol Chem 277(43): 40760-7.

Wertz, I. E. and V. M. Dixit "Signaling to NF-kappaB: regulation by ubiquitination." Cold Spring Harb Perspect Biol 2(3): a003350.

Weydert, C. J., T. A. Waugh, et al. (2006). "Overexpression of manganese or copper-zinc superoxide dismutase inhibits breast cancer growth." Free Radic Biol Med 41(2): 226-37.

Wiley, S. R., K. Schooley, et al. (1995). "Identification and characterization of a new member of the TNF family that induces apoptosis." Immunity 3(6): $673-82$.

Williams, T. M. and M. P. Lisanti (2005). "Caveolin-1 in oncogenic transformation, cancer, and metastasis." Am J Physiol Cell Physiol 288(3): C494-506.

Wilson, T. R., K. M. Redmond, et al. (2009). "Procaspase 8 overexpression in non-small-cell lung cancer promotes apoptosis induced by FLIP silencing." Cell Death Differ 16(10): 1352-61.

Wiseman, H. and B. Halliwell (1996). "Damage to DNA by reactive oxygen and nitrogen species: role in inflammatory disease and progression to cancer." Biochem J 313 ( Pt 1): 17-29.

Wong, C. H., K. B. Iskandar, et al. "Simultaneous induction of non-canonical autophagy and apoptosis in cancer cells by ROS-dependent ERK and JNK activation." PLoS One 5(4): e9996.

Wu, Y. T., S. Zhang, et al. (2008). "Signaling pathways from membrane lipid rafts to JNK1 activation in reactive nitrogen species-induced non-apoptotic cell death." Cell Death Differ 15(2): 386-97.

Yang, G., L. D. Truong, et al. (1999). "Caveolin-1 expression in clinically confined human prostate cancer: a novel prognostic marker." Cancer Res 59(22): 5719-23. 
Yang, J., L. J. Wu, et al. "Protein tyrosine kinase pathway-derived ROS/NO productions contribute to $\mathrm{G} 2 / \mathrm{M}$ cell cycle arrest in evodiamine-treated human cervix carcinoma HeLa cells." Free Radic Res 44(7): 792-802.

Yeh, W. C., A. Itie, et al. (2000). "Requirement for Casper (c-FLIP) in regulation of death receptor-induced apoptosis and embryonic development." Immunity 12(6): 633-42.

Yeh, W. C., A. Shahinian, et al. (1997). "Early lethality, functional NF-kappaB activation, and increased sensitivity to TNF-induced cell death in TRAF2deficient mice." Immunity 7(5): 715-25.

Ying, L. and L. J. Hofseth (2007). "An emerging role for endothelial nitric oxide synthase in chronic inflammation and cancer." Cancer Res 67(4): 140710.

Yu, R., S. Mandlekar, et al. (2000). "Tumor necrosis factor-related apoptosisinducing ligand-mediated apoptosis in androgen-independent prostate cancer cells." Cancer Res 60(9): 2384-9.

Zhang, A. Y., F. Yi, et al. (2006). "Lipid raft clustering and redox signaling platform formation in coronary arterial endothelial cells." Hypertension 47(1): 74-80.

Zhang, J., Y. Chen, et al. (2009). "Nuclear localization of C-FLIP-L and its regulation of AP-1 activity." Int J Biochem Cell Biol 41(8-9): 1678-84.

Zhang, W., B. Razani, et al. (2000). "Caveolin-1 inhibits epidermal growth factorstimulated lamellipod extension and cell migration in metastatic mammary adenocarcinoma cells (MTLn3). Transformation suppressor effects of adenovirus-mediated gene delivery of caveolin-1." J Biol Chem 275(27): 20717-25.

Zhou, Y., E. O. Hileman, et al. (2003). "Free radical stress in chronic lymphocytic leukemia cells and its role in cellular sensitivity to ROS-generating anticancer agents." Blood 101(10): 4098-104.

Zou, H., Y. Li, et al. (1999). "An APAF-1.cytochrome c multimeric complex is a functional apoptosome that activates procaspase-9." J Biol Chem 274(17): 11549-56. 


\title{
Chapter 2. Study 1: S-Nitrosylation of FLICE Inhibitory Protein Determines Its Interaction with RIP-1 and Activation of NF-KB
}

\author{
Siera Jo Talbott ${ }^{1,2}$, Sudjit Luanpitpong ${ }^{2}$, Christian Stehlik ${ }^{3}$, Liying Wang ${ }^{4}$, and \\ Yon Rojanasakul ${ }^{2}$ \\ ${ }^{1}$ Cancer Cell Biology Program and ${ }^{2}$ Department of Pharmaceutical Sciences, \\ West Virginia University, Morgantown, WV 26506, USA \\ ${ }^{3}$ Department of Medicine, Northwestern University, Chicago, IL 60611, USA \\ ${ }^{4}$ National Institute for Occupational Safety and Health, Morgantown, WV 26505, \\ USA
}

Corresponding Author:

Yon Rojanasakul

West Virginia University

Health Sciences Center

Morgantown, WV 26506

Tel: 3042931476

Fax: 3042932576

Email: yrojan@hsc.wvu.edu

Keywords: FLIP; S-nitrosylation; cancer; NF-kB; RIP1

Abbreviations: FLIP, Flice inhibitory protein; DED, death effector domains; NO, nitric oxide; DR, death receptor; NF-кB, nuclear factor kappa B; RIP1, receptor interacting protein 1; IKK $\gamma$, inhibitor of kappa B kinase gamma; PTM, posttranslational modification; iNOS, inducible nitric oxide synthase 


\section{Abstract}

Death receptor (DR) ligation can lead to divergent signaling pathways causing either caspase-mediated cell death or cell proliferation and inflammation. These variations in cellular fate are determined by adaptor proteins that are recruited to the DR signaling complex. Flice inhibitory protein (FLIP) is an established inhibitor of caspase-8-mediated apoptosis, and it is also involved in NF- $\kappa B$ activation. However, the molecular mechanism that regulates FLIP within this complex is unknown. In this study, we provide new evidence for the regulation of NF-kB by FLIP through S-nitrosylation which involves covalent modification of the protein's cysteine thiol by nitric oxide to form S-nitrosothiol. Point mutations of FLIP at cysteine residues 254 and 259 prevent FLIP Snitrosylation and its ability to activate NF-kB. The mechanism by which FLIP nitrosylation regulates NF-kB activity involves RIP1 binding and redistribution, whereas TRAF2 binding and distribution are unaffected. We further show that FLIP processing and cleavage is dependent on its nitrosylation status. Collectively, our study reveals a novel pathway for FLIP regulation of NF-kB through protein S-nitrosylation, which is a key posttranslational mechanism controlling DR-mediated cell death and survival. Since increased expression of FLIP and nitric oxide are frequently observed in chemotherapy-resistant tumors, S-nitrosylation of FLIP could be a key mechanism of chemoresistance and tumor growth. 


\section{Introduction}

Cellular proliferation, inflammation and apoptosis are highly regulated cellular processes important in homeostasis. Dysregulation of these processes can cause a multitude of human diseases including cardiovascular disease, autoimmune disorders and cancer. This occurs frequently in response to aberrant signaling initiated by death receptors (DRs). DRs belong to the tumor necrosis family receptor (TNFR) superfamily, which includes TNF receptors (TNFRs), Fas and TNF related apoptosis inducing ligand receptors (TRAIL-Rs) (Baker and Reddy 1998). DRs have been widely noted to play an important role in cancer development and metastasis (Debatin and Krammer 2004). DR ligands are pleiotropic cytokines that initiate the formation of signaling complexes on the cytoplasmic tail of the receptors. Contrary to their name, DRs can signal both for cell survival and cell death (Budd 2002). Receptor ligation results in receptor oligomerization and recruitment of adaptor proteins to the receptor complex, which determines the fate of the cell. Regulation of proteins that are recruited to the signaling complex is equally important in determining the outcome of DR stimulation. An example of a signaling complex that forms at the membrane is the death inducing signaling complex (DISC), where the adaptor protein FADD and procaspase-8 are recruited, resulting in caspase activation and subsequent cell death. DR-mediated cell survival however is mediated by an alternate complex that involves proteins upstream of nuclear factor kappa B (NF-kB), mitogen-activated protein kinase (MAPK) and phosphatidylinositol 3kinase (PI3K) pathways. 
Many cancer cells constitutively express DRs resulting in an increased sensitivity to ligands such as Fas and TRAIL (Gibson, Oyer et al. 2000). This increased sensitivity was thought to provide a selective opportunity to target cancer cells for apoptosis sparing normal cells with basal DR expression levels. This has led to the current exploration of adjuvant TRAIL therapies alongside chemotherapy in a wide variety of cancer treatments (Falschlehner, Ganten et al. 2009). However, many advanced cancers have gained resistance to apoptotic signaling via the upregulation of apoptotic inhibitor proteins such as cellular inhibitors of apoptosis (clAPs) and Flice inhibitor protein (FLIP) (Scaffidi, Schmitz et al. 1999). FLIP, originally noted for its importance in lymphocyte proliferation and development (Thome and Tschopp 2001), is a known inhibitor of DRmediated apoptosis (Krueger, Baumann et al. 2001) Multiple isoforms of FLIP mRNA have been identified, but two protein forms, FLIP (long) and FLIP (short) are most readily observed, each having distinct signaling effects at the DISC (Wajant 2003). FLIPL is a $55 \mathrm{kDa}$ protein that is a substrate of caspase-8 resulting in truncation from the C-terminal end into a $43 \mathrm{kDa}$ protein which has been noted to be more active (Kataoka and Tschopp 2004; Dohrman, Kataoka et al. 2005). FLIPS is a $22 \mathrm{kDa}$ isoform consisting only of the two $\mathrm{N}$-terminal death effector domains (DEDs). Both isoforms of FLIP inhibit apoptosis via their ability to form heterodimers with procaspase-8, preventing activation of the caspase cascade (Irmler, Thome et al. 1997; Wajant 2003). Elevated FLIP expression levels are directly associated with chemotherapy-resistant cancers as an acquired mechanism to evade DR-mediated apoptosis (Wajant 2003). 
Additionally, FLIP has been reported in other DR pathways, including the activation of the potent pro-inflammatory transcription factor NF-кB (Kataoka, Budd et al. 2000; Sun, Zachariah et al. 2003). FLIP can interact with multiple proteins important in TNF-mediated NF-кB activation including receptor interacting protein kinase 1 (RIP1) (Chaudhary, Eby et al. 2000), TNFRassociated factor 2 (TRAF2) (Kataoka and Tschopp 2004), and inhibitor kappa B kinase gamma (IKK $\gamma$ ) (Golks, Brenner et al. 2006), indicating the multiple roles for FLIP in NF-kB signaling. Furthermore, NF-kB promotes FLIP expression in a positive feedback loop (Kreuz, Siegmund et al. 2001). These high levels of FLIP can potentially enhance a chronic inflammatory response and promote tumor growth.

Abundant concentrations of DR-ligands are produced in response to infection, inflammation and in response to chemotherapeutic drugs. NF- $\mathrm{KB}$ is sequestered by NF-KB Inhibitors (IKBs) in the cytosol under normal conditions. Proinflammatory cytokines and DR-ligands activate NF- $\mathrm{kB}$ by targeting IKK for activation leading to phosphorylation and subsequent degradation of $\mathrm{I}_{\kappa} \mathrm{B}$, thereby allowing NF- $\mathrm{KB}$ to translocate to the nucleus to function as a transcription factor. TNFR-mediated activation of NF-kB is initiated by the formation of a cytoplasmic complex containing TNFR superfamily $1 \mathrm{~A}$-associated via death domain (TRADD), RIP1, TRAF2 and clAPs. During TNFR signaling, RIP1 is polyubiquitinated by clAPs promoting the formation of a pro-survival complex upstream of NF-kB activation (Varfolomeev, Goncharov et al. 2008). RIP is essential for activation of IKKs by this complex (Devin, Cook et al. 2000). 
Inhibition or loss of clAPs, as well as the deubiquitination of RIP, causes apoptosis via RIP recruitment to the DISC (Geserick, Hupe et al. 2009). Therefore, RIP is a pivotal signaling protein in DR-stimulated complex formation. By regulating both cell death and proliferation it is not surprising that RIP overexpression has also been associated with poor prognosis in cancer (Park, Hatanpaa et al. 2009).

Reactive nitrogen and oxygen species are commonly found in pathologies such as chronic inflammation, cardiovascular disease and advanced tumorigenesis. Activation of inducible nitric oxide synthase (iNOS) and elevated levels of nitric oxide (NO), lead to rapid increases in the S-nitrosylation of proteins. S-nitrosylation is the covalent addition of NO to the thiol side chain of a cysteine residue. This post-translational modification (PTM) can regulate protein interactions and modify downstream signaling. Other PTMs have been reported to significantly alter FLIP stability and function (Higuchi, Yoon et al. 2003; Kaunisto, Kochin et al. 2009). We have previously shown that S-nitrosylation of FLIP can prevent its ubiquitination and subsequent degradation making it a more potent inhibitor of Fas-mediated apoptosis (Chanvorachote, Nimmannit et al. 2005).

Here we demonstrate the effects of FLIP S-nitrosylation of residues 254 and 259 on NF-kB activation. The underlying mechanism of changes in NF-kB activation is due to the ability of S-nitrosylated FLIP to bind RIP1 following TNF- $\alpha$ stimulation. Additionally, we demonstrate that S-nitrosylation of FLIP regulates its cleavage into shorter forms. Determining the regulation of FLIP signaling is 
fundamental to understanding its role in disease and is imperative for the future development of effective therapeutic strategies in chemotherapy-resistant cancers. 


\section{Experimental procedures}

Cell lines and reagents: HEK 293, MCF-7, and A549 cells were cultured in Dulbecco's modified Eagle's medium (Invitrogen, Carlsbad, CA) containing 10\% fetal bovine serum, 2 mM L-glutamine, 20 mM HEPES, 100 units $/ \mathrm{ml}$ penicillin, and $100 \mu \mathrm{g} / \mathrm{ml}$ streptomycin in a $5 \% \mathrm{CO}_{2}$ environment at $37^{\circ} \mathrm{C}$. Recombinant FasL (SuperFasL), monoclonal antibody against FLIP (Dave-2), and the NO donor DPTA NONOate were purchased from Alexis Biochem (San Diego, CA). The NO donor sodium nitroprusside (SNP), NO inhibitors amino-guanidine (AG) and 2-(4-carboxy-phenyl)-4,4,5,5 tetramethylimidazoline-1-oxy-3-oxide (PTIO), and Hoechst 33342 were from Sigma (St. Louis, MO). The transfection agent Lipofectamine Plus was purchased from Invitrogen (Carlsbad, CA). Monoclonal anti-cfp (3E6) antibody was purchased from Invitrogen (Carlsbad, CA). Anti-myc antibodies were purchased from Millipore (Billerica, MA) and SCBT (Santa Cruz, CA). Polyclonal antibody against GAPDH was purchased from BD Transduction (San Jose, CA). Polyclonal antibody against FLAG (OctA) was purchased from SCBT (Santa Cruz, CA). Polyclonal antibody against TRAF2 was purchased from Cell Signaling Technology (Danvers, MA). Commercial protease inhibitor mixture was purchased from Roche Applied Science (Indianapolis, IN). pRL and pGL vectors were purchased from Promega (Madison, WI). pcDNA3-FLIP plasmids were made as described previously (Chanvorachote, Nimmannit et al. 2005). pECFP-TRAF2 was made as described previously and provided as a kind gift from J. Schmid (Medical University Vienna, Austria) (Birbach, Gold et al. 2002). 
S-nitrosylation assay: HEK 293 cells were transfected with pcDNA3-FLIP. The following day cells were treated as indicated in figure legend. S-Nitrosylated protein detection assay kit was commercially obtained from Cayman Chemical Company (Ann Arbor, MI) and was used according to the manufacturer's instruction. Briefly, cell pellets were lysed under the protein free thiols blocking condition. Protein S-nitrosothiols were then reduced to yield free thiols which were covalently labeled with maleimide-biotin. Lysate protein $(500 \mu \mathrm{g})$ was subjected to the immunoprecipitation as described earlier. Subsequent analysis for S-nitrosylated protein was determined by Western blotting using Snitrosylation detection reagent I (HRP) and by fluorescence reading using Snitrosylation detection reagent II (fluorescein). For Western blot analysis, protein samples were prepared in Laemmli buffer and were resolved under denaturing conditions. For fluorescence reading, immune complexes were further incubated with fluorescein detection agent overnight following the standard immunoprecipitation step. Complexes were rinsed with wash buffer three times, resuspended in colorless Laemmli sample buffer, and boiled for five minutes. Clear lysates were loaded onto the fluorescence plate and analyzed for fluorescence intensity using a fluorescence plate reader at a 485-nm excitation and a 520-nm emission (FLUOstar OPTIMA, BMG Labtech, Durham, NC).

NF- $\kappa B$ gene reporter assay: $1.5 \times 10^{5} \mathrm{HEK} 293$ cells/well were seeded on $0.02 \%$ gelatin coated 24-well plates. Cells were transfected the following day with 600 
ng of empty pcDNA3 vector, the indicated amount of pcDNA3-FLIP plasmids, $100 \mathrm{ng}$ of a multimeric NF- $\mathrm{kB}$ pGL2 luciferase vector and $5 \mathrm{ng}$ of the Renilla pRLTK vector. Transfections were performed using Lipofectamine 2000 (Invitrogen). Expression of proteins was verified by immunoblotting. Media was changed 6 hours after transfection and cells were grown overnight. Luciferase activity was determined using a dual-luciferase reporter assay system kit (Promega, San Luis Obispo, CA). The activity of the NF- ${ }_{k} \mathrm{~B}$ reporter luciferase was standardized to that of Renilla luciferase. Within the same experiment, each transfection was performed in quadruplicate, and where necessary, empty control plasmid was added to keep each transfection receiving the same amount of total DNA. Data shown are representative averages and standard deviations from one of three separate experiments performed.

Apoptosis assay: MCF-7 cells were transfected with FLIP wildtype or FLIP 2CM using Amaxa Nucleofector (Lonza, Walkersville, MD) using the recommended manufacturer protocol. Transfected cells were seeded at 15,000 cells per well in a 96 well plate and allowed to recover for 24 hours. Cells were treated with 200 $\mathrm{ng} / \mathrm{mL}$ FasL or $100 \mathrm{ng} / \mathrm{mL}$ TNF- $\alpha$ for 12 hours. Apoptosis was determined by Hoechst 33342 DNA fragmentation assay. Briefly, cells were incubated with 10 $\mu \mathrm{g} / \mathrm{mL}$ of Hoechst 33342 for $30 \mathrm{~min}$ and were visualized under a fluorescence microscope (Leica Microsystems, Bannockburn, IL). Cells having intensely condensed and/or fragmented nuclei were considered as apoptotic. 
Immunoblotting: Cells were lysed in RIPA lysis buffer containing $1 \mathrm{mM}$ sodium orthovanadate, $100 \mathrm{mM}$ phenylmethylsulfonyl fluoride, and a commercial protease inhibitor mixture (Roche Applied Science, Indianapolis, IN) for 20 min on ice. After insoluble debris was pelletted by centrifugation at $14,000 \times \mathrm{g}$ for 10 min at $4{ }^{\circ} \mathrm{C}$, the supernatants were collected and determined for protein content using the Bradford method (Bio-Rad). Proteins $(60 \mu \mathrm{g})$ were resolved on a reducing $10 \%$ SDS-polyacrylamide gel and transferred onto PVDF membranes. The transferred membranes were blocked for 1 hour in $5 \%$ nonfat dry milk in Tris-buffered Tween (25 mM Tris- $\mathrm{HCl}, \mathrm{pH} 7.4,125 \mathrm{mM} \mathrm{NaCl}, 0.05 \%$ Tween 20) and incubated with the appropriate primary antibodies. Membranes were washed 3 times with Tris-buffered Tween for $10 \mathrm{~min}$ and incubated with horseradish peroxidase-coupled isotype-specific secondary antibodies for 1 hour at room temperature. The immune complexes were detected by enhanced chemiluminescence $(E C L)$ detection.

Immunoprecipitation: Cells were lysed in lysis buffer containing $20 \mathrm{mM}$ Tris- $\mathrm{HCl}$, $\mathrm{pH} 7.5,1 \%$ Triton $\mathrm{X}-100,150 \mathrm{mM} \mathrm{NaCl}, 10 \%$ glycerol, $1 \mathrm{mM}$ sodium orthovanadate, $50 \mathrm{mM}$ sodium fluoride, $100 \mathrm{mM}$ phenylmethylsulfonyl fluoride, and a protease inhibitor mixture for $20 \mathrm{~min}$ on ice. Immunoprecipitation experiments were carried out using Dynabeads (Invitrogen, Carlsbad, CA) and the recommended protocol. Immunoblotting was carried out as described above. 
Immunofluoresence: Cells were seeded on rat type I collagen coated coverslips $\left(5 \mu \mathrm{g} / \mathrm{cm}^{2}\right)$, fixed with $3.7 \%$ paraformaldehyde for $15 \mathrm{~min}$, incubated in $50 \mathrm{mM}$ glycine for $5 \mathrm{~min}$, and permeabilized and blocked with $0.5 \%$ saponin, $1.5 \%$ BSA, and $1.5 \%$ normal goat serum for 30 min. FLIP was immunostained with anti-myc, RIP1 was immunostained with an antibody against FLAG (OctA). TRAF2 was immunostained with antibody against CFP. Secondary Alexa Fluor 405, 488,546, and 647-conjugated antibodies, and phalloidin were used from Invitrogen (Carlsbad, CA). Cells were washed with PBS containing 0.5\% saponin, and coverslips were mounted using Fluoromount-G (Southern Biotechnology Associates, Birmingham, AL). Cells were viewed with a Zeiss LSM 510 confocal on an Axiolmager Z1 microscope using a 63x objective lens.

Statistics: The differences between treatment groups were analyzed using an unpaired Student's t-test and were considered significant when $P<0.05$. Error bars display standard deviation.

Acknowledgements: Imaging experiments were performed in the West Virginia University Microscope Imaging Facility, which is supported in part by the Mary Babb Randolph Cancer Center and NIH grant P20 RR016440. We thank Johannes A. Schmid for generously sharing plasmids. This work was financially supported by the American Heart Association (predoctoral fellowship 0715376B to ST) and the National Institutes of Health (R01-HL076340 to YR). 


\section{Results}

FLIP is S-nitrosylated at residues 254 and 259

The antiapoptotic role of FLIP in DR-mediated apoptotic signaling is firmly established. We have previously reported that FLIP S-nitrosylation prevents its ubiquitination and subsequent degradation, thereby making it a more potent inhibitor of Fas-mediated apoptosis (Chanvorachote, Nimmannit et al. 2005). Since DR signaling can also lead to cell survival we tested the hypothesis that Snitrosylation of FLIP modulates its role as an NF-kB activator. We used our previously described double cysteine mutant of FLIP (2CM), where single point mutations of cysteine residues 254 and 259 to alanines in the caspase-like domain (CLD) of FLIP prevent S-nitrosylation of FLIP (Figure 1a) (Chanvorachote, Nimmannit et al. 2005). Transiently transfected FLIP 2CM showed impaired responsiveness to pharmacological NO modification, as evident by the lower overall S-nitrosylation of FLIP 2CM compared to wild type FLIP, with S-nitrosylation levels comparable to those detectable following the treatment with the iNOS inhibitor amino-guanidine (AG) (Figures $1 \mathrm{~b}$ and $\mathrm{c}$ ). We do not see a complete reduction in S-nitrosylation levels since there are additional cysteine residues within the FLIP protein sequence that could potentially be Snitrosylated. 
S-nitrosylation of FLIP modulates NF- $\kappa B$ activation

It is well established that FLIP can promote NF-kB activation (Chaudhary, Eby et al. 2000; Kataoka and Tschopp 2004; Golks, Brenner et al. 2006). While transient transfection of wildtype FLIP activates NF-kB in a dose dependent manner compared to empty vector control (Figure 2a), FLIP 2CM completely lacks any detectable activation of NF-kB (Figure $2 \mathrm{c}$ ). This result suggests that the S-nitrosylation of FLIP at residues 254 and 259 is necessary for FLIPLmediated activation of NF-kB. The expression of all FLIP constructs has been verified by immunoblot (Figures $2 \mathrm{~b}$ and $\mathrm{d}$ ). In agreement with previous results, transient expression of the two N-terminal DEDs, representative of FLIPs, but not one N-terminal DED alone, independently activates NF-kB (Figure 2c) (Kataoka and Tschopp 2004; Golks, Brenner et al. 2006). These results indicate that Snitrosylation of FLIP at cysteine residues 254 and 259 represents a distinct mechanism to activate NF-kB, compared to the DED-mediated response previously reported. To further examine the role of S-nitrosylation of FLIP on NF$\kappa \mathrm{B}$ activation, we performed experiments using NO modifying reagents. Cells were transiently transfected with wild type FLIP expression constructs, followed by treatment with the NO donor DPTA NONOate (NONO) and sodium nitroprusside (SNP). NO supplementation using either NONO or SNP treatment caused a decrease in FLIP-mediated NF- $\mathrm{kB}$ activation, which was independent on the expression level of FLIP, while FLIP 2CM was not affected by NO supplementation and failed to activate NF-KB (Figure 2e). Although we expected to observe an increase in NF-kB activation, the subunits of NF-kB are also 
targets for S-nitrosylation, which results in an overall inhibition of its activation by preventing translocation to the nucleus and interfering with DNA binding (Marshall, Hess et al. 2004). However, we show the FLIP-mediated induction of NF-kB remains statistically significant compared to control cells (Figure 2e). Additionally, NF-кB activation promoted by transient transfection of FLIP, but not FLIP 2CM was significantly increased upon global NO inhibition using the NO scavenger 2-(4-carboxy-phenyl)-4,4,5,5 tetramethylimidazoline-1-oxy-3-oxide (PTIO) and the selective iNOS inhibitor amino-guanidine (AG) (Figure 2f). Thus, our results show that the S-nitrosylation of FLIP is upstream of NF-kB activation and is another S-nitrosylation-mediated mechanism to modulate NF-kB activation.

Since DRs signal for NF-kB activation and apoptosis, we also determined the effect of S-nitrosylation of FLIP on apoptosis. Cells were transfected with either an empty vector, FLIP or FLIP 2CM, followed by treatment with the DR agonist TNF- $\alpha$ or FasL and quantification of apoptosis. Treatment of the cells with TNF- $\alpha$ and FasL causes increased cell death shown by an increase in fragmented nuclei, which is inhibited by FLIP, but not FLIP 2CM (Figures 3a and b). These results indicate that the S-nitrosylation of FLIP is important in both proliferative and apoptotic signaling originating from DRs.

S-nitrosylation of FLIP modulates RIP1 binding and localization

To further delineate the molecular mechanism by which S-nitrosylation of FLIP mediates NF-kB activation, we investigated its interaction with upstream 
adaptor RIP1, which is a crucial signaling protein upstream of NF-kB activation and known binding partner of FLIP (Irmler, Thome et al. 1997). We transiently transfected cells with FLIP or FLIP $2 \mathrm{CM}$ and tested the interaction with endogenous RIP1. FLIP or FLIP 2CM was immunoprecipitated and the immune complexes were probed for RIP1. FLIP binds to RIP1 in the absence of any DR stimulation, and the interaction is disrupted following TNF- $\alpha$ treatment (Figure 4a). In contrast, FLIP 2CM retained its ability to interact with RIP1 even in cells that were treated with TNF- $\alpha$ (Figure 4a) at the same level as observed in cells not stimulated by TNF- $\alpha$ (data not shown). This differential binding indicates that the S-nitrosylation of FLIP at residues 254 and 259 inhibits binding to RIP1. Furthermore, FLIP, but not FLIP 2CM is cleaved upon TNF- $\alpha$ treatment, also indicating that S-nitrosylation is necessary for FLIP processing. We next examined the cellular localization of FLIP and RIP1 by confocal microscopy in HEK 293 cells, which express very low levels of endogenous FLIP (data not shown). We observed that in resting cells, RIP1 is distributed in the cytoplasm however it is recruited to the membrane in response to TNF- $\alpha$ stimulation. This translocation to the membrane is impaired, when the FLIP 2CM mutant is coexpressed (Figure 4b). These results suggest that the S-nitrosylation of FLIP is essential in regulating its interaction with RIP1, thus affecting localization of RIP1 to the DR complex following receptor ligation, thereby modulating DR signaling.

TRAF2 localization and FLIP binding is not dependent on FLIP S-nitrosylation 
TRAF2 is another DR signaling complex adaptor protein, which is essential for NF-kB activation (Devin, Cook et al. 2000). Since the Snitrosylation of FLIP affects its interaction with RIP1, we also examined the interaction of FLIP with TRAF2. We transiently transfected cells with either FLIP or FLIP 2CM, immunoprecipitated FLIP and analyzed the immune complexes for the presence of endogenous TRAF2. However, in contrast to the FLIP interaction with RIP1, its interaction with TRAF2 was not affected, when comparing FLIP and FLIP 2CM, either in the presence or absence of TNF- $\alpha$ (Figure 5a). Additionally, we examined the localization of TRAF2 in HEK 293 cells following TNF- $\alpha$ stimulation. We found that TRAF2 is distributed in the cytoplasm in resting cells and translocates to the membrane following receptor stimulation with TNF- $\alpha$ regardless of the presence of FLIP or FLIP 2CM (Figure 5b). Thus, our results indicate that S-nitrosylation of FLIP at residues 254 and 259 does not affect its interaction with TRAF2 and therefore is expected to not cause the effects on NF-kB activation.

S-nitrosylation of FLIP causes processing into shorter forms

FLIP protein levels and the ratio of FLIP $L$ and FLIPS vary in different cell types. It is also known that $F L I P L$ is rapidly converted into a p43 fragment through proteolytic cleavage (Neumann, Pforr et al.; Kataoka and Tschopp 2004; Dohrman, Kataoka et al. 2005). Since different forms of FLIP show distinct effects on NF-kB activation (Figure 2c), we next tested whether DR ligation causes proteolytic processing of FLIP, which could also impact activation of NF- 
$\kappa B$. We used a human lung adenocarcinoma A549 cell line, which expresses a high level of FLIP, and either mock treated the cells, or treated the cells with the DR-ligands TNF- $\alpha$ and FasL for different times in the absence or presence of the proteasome inhibitor MG132. We then probed the lysates for expression of endogenous FLIP. We found that the $55 \mathrm{kDa} F L I P L$ is partially converted in resting cells into the $43 \mathrm{kDa}$, C-terminally truncated FLIPL (Figure 6a). Treatment with MG132 also revealed presence of p22 FLIP, which is proteasomally degraded. However, treatment with either TNF- $\alpha$ or FasL enhanced expression or stability of FLIPs, with FasL also promoting further processing of p43 into the p39 version, which is yet another C-terminal processing event. We next tested whether S-nitrosylation could impact proteolytic processing of FLIP. We transiently transfected HEK 293 cells with either FLIP or FLIP 2CM, treated cells with FasL or TNF- $\alpha$, and probed cell lysates for FLIP. While FLIP is partially converted into p43, as observed for endogenous FLIP, FLIP 2CM is protected from processing and remains as p55 FLIPL (Figures $6 \mathrm{~b}$ and $\mathrm{c}$ ). Additionally FLIP processing is enhanced by NO supplementation by treatment with NONO comparable to FasL treatment (Figure 6d). This increased processing is also consistent with S-nitrosylation fragments seen in Figure 1c. Thus, our data indicate that S-nitrosylation at residues 254 and 259 is required for the ability of FLIPL to act as a substrate and its proteolytic conversion into p43. 


\section{Discussion}

In this study we demonstrate that S-nitrosylation of FLIP at cysteines 254 and 259 mediate RIP1 binding to induce NF-kB activation. This mechanism is distinct from previously reported FLIP-mediated NF-kB activity, thus FLIP Snitrosylation provides a novel molecular mechanism of FLIP-mediated NF-kB regulation (Kataoka and Tschopp 2004; Golks, Brenner et al. 2006). FLIP can interact with multiple proteins upstream of NF-kB and therefore it is not surprising to discover that differences in FLIP signaling and nitrosylation state can lead to the activation of NF-kB via multiple avenues. An increasing number of studies have been reported examining the inhibition of FLIP using small molecule inhibitors, chemicals or siRNA techniques (Bijangi-Vishehsaraei, Saadatzadeh et al.; Sanchez-Perez, Ortiz-Ferron et al.; Luo, Wang et al. 2008; Wilson, McEwan et al. 2009), however, additional regulation may be found within the cell by PTMs.

FLIP PTMs have been shown to be involved in changes in its stability, binding partner interactions and localization. FLIP phosphorylation prevents localization to the DISC in TRAIL-mediated DR5 signaling and leads to an increase in TRAIL mediated apoptosis (Higuchi, Yoon et al. 2003). We have previously reported that FLIP S-nitrosylation prevents ubiquitination and subsequent degradation (Chanvorachote, Nimmannit et al. 2005). S-nitrosylation has gained increasing importance as a PTM that affects protein interactions and signaling pathways. The tumor environment frequently has elevated NO levels due to constitutive iNOS activity and redox imbalances, leading to an overall 
increase in protein nitrosylation. In this study, we demonstrate NF- $\mathrm{kB}$ induction by wildtype and nitrosylable FLIP in a dose-dependent manner. However, NF-kB activation is completely abrogated, when testing a mutant of FLIP that cannot be nitrosylated at residues 254 and 259. Rescue of NF-kB activity by FLIPS (FLIP 2DED) indicates there are at least two separate mechanisms for FLIP-mediated $\mathrm{NF}-\mathrm{\kappa B}$ activation in agreement with previous reports that FLIP isoforms have distinct signaling, because the nitrosylation sites we investigated map within the caspase-like domain and not the DED (Krueger, Schmitz et al. 2001; Wajant 2003). NO supplementation results in an increase in overall protein nitrosylation, however we observed a decrease in NF-kB activity, contrary to the expected increase from additionally S-nitrosylated FLIP. Others have shown NF-kB activity is inhibited when subunit p50 is S-nitrosylated due to its inability to bind DNA (Marshall and Stamler 2001). Interestingly, our results show FLIP significantly induces NF-KB activation even with NO inhibition. FLIP has most notably been reported as an inhibitor of apoptosis by binding procaspase- 8 thereby preventing its activation (Irmler, Thome et al. 1997; Krueger, Baumann et al. 2001). We further demonstrate that S-nitrosylation at residues 254 and 259 are necessary to confer this resistance to apoptosis.

It has been previously reported that FLIP interacts specifically with TRAF2 to activate NF-kB (Kataoka and Tschopp 2004). We were unable to demonstrate that the S-nitrosylation of FLIP at residues 254 and 259 alters the interaction with TRAF2, indicating that this interaction is not responsible for the change in Snitrosylated FLIP-mediated NF- $\mathrm{B}$ activation. RIP1 is another essential signaling 
protein for TNFR-mediated NF-kB activation (Kelliher, Grimm et al. 1998). The ubiquitination state of RIP is mediated by clAPs, which determine the formation of the DISC or NF-kB signaling complex (Geserick, Hupe et al. 2009). Our results demonstrate that the S-nitrosylation of FLIP at residues 254 and 259 mediate RIP binding and localization. Our results indicate that the altered interaction between FLIP and RIP1 as a consequence from S-nitrosylation, contributes to the mechanism for FLIP-mediated NF-kB activation. With nonnitrosylable FLIP retaining RIP binding, thus preventing RIP1 recruitment to DRs, $\mathrm{NF}-\mathrm{kB}$ is unable to be canonically activated. Protein sequestration is a common means for controlling signal transduction. For example, compartmentalization by lipid rafts changes the makeup of TNFR1 and Fas signaling complexes (Schutze, Tchikov et al. 2008).

Varying FLIP mRNA transcripts correspond to FLIPL and FLIPS and have been reported to have varying affinity in binding partners and distinctive downstream signaling, in addition to the classical anti-apoptotic function through caspase-8 inhibition (Park, Kim et al. 2001; Kim, Park et al. 2008). FLIPL has been shown to be a caspase- 8 substrate resulting in a $43 \mathrm{kDa}$ protein with more robust NF-kB activation (Kataoka and Tschopp 2004; Dohrman, Kataoka et al. 2005). We identified in FasL and TNF- $\alpha$ treated A549 cells, which express high levels of FLIP, additional cleavage fragments. Additionally, we demonstrate that FLIP $\mathrm{L}$ is being cleaved into smaller fragments by using a myc-tagged expression construct that includes only the exon regions of the coding sequence thereby preventing the smaller fragments from being mRNA splice variants. Given that 
different forms of FLIP have different signaling capabilities, this could have dramatic implications in chemotherapy-resistant cancer cells that have elevated levels of FLIP in conjunction with high levels of caspase-8. Ligand-induced cleavage and multiple cleavage truncations of FLIP have not been previously reported, and it is currently unknown if caspase- 8 is responsible for the additional cleavage fragments. Additionally, the increases in NO associated with disease could further impact this signaling pathway, since FLIP is subject to nitrosylation. The S-nitrosylation status of FLIP likely affect proteolytical processing of FLIP, since we were unable to detect cleavage in our FLIP 2CM transfected cells.

Our data could suggest that S-nitrosylation of FLIP leads to an increase in NF-kB activity in two distinct ways. First, S-nitrosylation of FLIP results in an increase in FLIP processing thereby generating a p22 fragment which is a known inducer of NF-kB (Golks, Brenner et al. 2006). The FLIP 2CM was unable to be processed into the p22 fragment and therefore would be unable to induce NF-kB through this mechanism. Second, FLIP S-nitrosylation could lead to the disassociation of binding with RIP1 potentially promoting RIP1's activation of NFКB. The FLIP 2CM retained RIP1 binding potentially interfering with RIP1mediated NF-kB activation. However, there is still a signaling paradox in this system of NO-signaling as TNF- $\alpha$-stimulation results in an overall decrease in Snitrosylation of FLIP as well as an overall decrease in NO production (data not shown). The specific details of S-nitrosylation are reported to be exceedingly complex and the mechanisms of targeted specificity still remains unclear 
(Tannenbaum and White 2006). We clearly demonstrate S-nitrosylation of FLIP affects FLIP function, however it probably does not affect all FLIP interactions.

In summary, many chemotherapy-resistant tumors are insensitive to cell death through elevated levels of FLIP and consequently elevated NF-kB activity. Targeted FLIP silencing has been shown to restore sensitivity to DR-mediated apoptosis (Sharp, Lawrence et al. 2005). Studies on FLIP regulation by PTMs and resulting interactions are imperative to better understand its role in normal physiology and pathogenesis of cancer and inflammation. Specifically our results suggest that developing strategies to interfering with FLIP S-nitrosylation could have potential for future targeted therapies for chemotherapy-resistant tumors. 


\section{References :}

Baker, S. J. and E. P. Reddy (1998). "Modulation of life and death by the TNF receptor superfamily." Oncogene 17(25): 3261-70.

Bijangi-Vishehsaraei, K., M. R. Saadatzadeh, et al. "4-(4-Chloro-2methylphenoxy)-N-hydroxybutanamide (CMH) targets mRNA of the c-FLIP variants and induces apoptosis in MCF-7 human breast cancer cells." Mol Cell Biochem 342(1-2): 133-42.

Birbach, A., P. Gold, et al. (2002). "Signaling molecules of the NF-kappa B pathway shuttle constitutively between cytoplasm and nucleus." Chem 277(13): 10842-51.

Budd, R. C. (2002). "Death receptors couple to both cell proliferation and apoptosis." J Clin Invest 109(4): 437-41.

Chanvorachote, P., U. Nimmannit, et al. (2005). "Nitric oxide negatively regulates Fas CD95-induced apoptosis through inhibition of ubiquitin-proteasomemediated degradation of FLICE inhibitory protein." J Biol Chem 280(51): 42044-50.

Chaudhary, P. M., M. T. Eby, et al. (2000). "Activation of the NF-kappaB pathway by caspase 8 and its homologs." Oncogene 19(39): 4451-60.

Debatin, K. M. and P. H. Krammer (2004). "Death receptors in chemotherapy and cancer." Oncogene 23(16): 2950-66.

Devin, A., A. Cook, et al. (2000). "The distinct roles of TRAF2 and RIP in IKK activation by TNF-R1: TRAF2 recruits IKK to TNF-R1 while RIP mediates IKK activation." Immunity 12(4): 419-29.

Dohrman, A., T. Kataoka, et al. (2005). "Cellular FLIP (long form) regulates CD8+ T cell activation through caspase-8-dependent NF-kappa B activation." $\underline{\mathrm{J}}$ Immunol 174(9): 5270-8.

Falschlehner, C., T. M. Ganten, et al. (2009). "TRAIL and other TRAIL receptor agonists as novel cancer therapeutics." Adv Exp Med Biol 647: 195-206. 
Geserick, P., M. Hupe, et al. (2009). "Cellular IAPs inhibit a cryptic CD95-induced cell death by limiting RIP1 kinase recruitment." J Cell Biol 187(7): 1037-54.

Gibson, S. B., R. Oyer, et al. (2000). "Increased expression of death receptors 4 and 5 synergizes the apoptosis response to combined treatment with etoposide and TRAIL." Mol Cell Biol 20(1): 205-12.

Golks, A., D. Brenner, et al. (2006). "The c-FLIP-NH2 terminus (p22-FLIP)

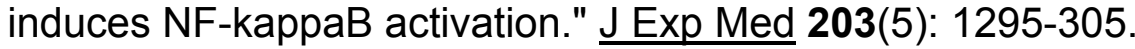

Higuchi, H., J. H. Yoon, et al. (2003). "Bile acids stimulate cFLIP phosphorylation enhancing TRAIL-mediated apoptosis." J Biol Chem 278(1): 454-61.

Irmler, M., M. Thome, et al. (1997). "Inhibition of death receptor signals by cellular FLIP." Nature 388(6638): 190-5.

Kataoka, T., R. C. Budd, et al. (2000). "The caspase-8 inhibitor FLIP promotes activation of NF-kappaB and Erk signaling pathways." Curr Biol 10(11): 640-8.

Kataoka, T. and J. Tschopp (2004). "N-terminal fragment of c-FLIP(L) processed by caspase 8 specifically interacts with TRAF2 and induces activation of the NF-kappaB signaling pathway." Mol Cell Biol 24(7): 2627-36.

Kaunisto, A., V. Kochin, et al. (2009). "PKC-mediated phosphorylation regulates C-FLIP ubiquitylation and stability." Cell Death Differ 16(9): 1215-26.

Kelliher, M. A., S. Grimm, et al. (1998). "The death domain kinase RIP mediates the TNF-induced NF-kappaB signal." Immunity 8(3): 297-303.

Kim, D. J., C. Park, et al. (2008). "Association of TRAF2 with the short form of cellular FLICE-like inhibitory protein prevents TNFR1-mediated apoptosis." J Mol Signal 3: 2.

Kreuz, S., D. Siegmund, et al. (2001). "NF-kappaB inducers upregulate cFLIP, a cycloheximide-sensitive inhibitor of death receptor signaling." Mol Cell Biol 21(12): 3964-73. 
Krueger, A., S. Baumann, et al. (2001). "FLICE-inhibitory proteins: regulators of death receptor-mediated apoptosis." Mol Cell Biol 21(24): 8247-54.

Krueger, A., I. Schmitz, et al. (2001). "Cellular FLICE-inhibitory protein splice variants inhibit different steps of caspase-8 activation at the CD95 deathinducing signaling complex." J Biol Chem 276(23): 20633-40.

Luo, A., W. Wang, et al. (2008). "Short hairpin RNA targeting c-FLIP sensitizes human cervical adenocarcinoma Hela cells to chemotherapy and radiotherapy." Cancer Lett 271(2): 323-32.

Marshall, H. E., D. T. Hess, et al. (2004). "S-nitrosylation: physiological regulation of NF-kappaB." Proc Natl Acad Sci U S A 101(24): 8841-2.

Marshall, H. E. and J. S. Stamler (2001). "Inhibition of NF-kappa B by Snitrosylation." Biochemistry 40(6): 1688-93.

Neumann, L., C. Pforr, et al. "Dynamics within the CD95 death-inducing signaling complex decide life and death of cells." Mol Syst Biol 6: 352.

Park, S., K. J. Hatanpaa, et al. (2009). "The receptor interacting protein 1 inhibits p53 induction through NF-kappaB activation and confers a worse prognosis in glioblastoma." Cancer Res 69(7): 2809-16.

Park, S. J., Y. Y. Kim, et al. (2001). "Alternative splicing variants of c-FLIP transduce the differential signal through the Raf or TRAF2 in TNF-induced cell proliferation." Biochem Biophys Res Commun 289(5): 1205-10.

Sanchez-Perez, T., G. Ortiz-Ferron, et al. "Mitotic arrest and JNK-induced proteasomal degradation of FLIP and Mcl-1 are key events in the sensitization of breast tumor cells to TRAIL by antimicrotubule agents." Cell Death Differ 17(5): 883-94.

Scaffidi, C., I. Schmitz, et al. (1999). "The role of c-FLIP in modulation of CD95induced apoptosis." J Biol Chem 274(3): 1541-8.

Schutze, S., V. Tchikov, et al. (2008). "Regulation of TNFR1 and CD95 signalling by receptor compartmentalization." Nat Rev Mol Cell Biol 9(8): 655-62. 
Sharp, D. A., D. A. Lawrence, et al. (2005). "Selective knockdown of the long variant of cellular FLICE inhibitory protein augments death receptormediated caspase-8 activation and apoptosis." J Biol Chem 280(19): 19401-9.

Sun, Q., S. Zachariah, et al. (2003). "The human herpes virus 8-encoded viral FLICE-inhibitory protein induces cellular transformation via NF-kappaB activation." J Biol Chem 278(52): 52437-45.

Tannenbaum, S. R. and F. M. White (2006). "Regulation and specificity of Snitrosylation and denitrosylation." ACS Chem Biol 1(10): 615-8.

Thome, M. and J. Tschopp (2001). "Regulation of lymphocyte proliferation and death by FLIP." Nat Rev Immunol 1(1): 50-8.

Varfolomeev, E., T. Goncharov, et al. (2008). "C-IAP1 and c-IAP2 are critical mediators of tumor necrosis factor alpha (TNFalpha)-induced NF-kappaB activation." J Biol Chem 283(36): 24295-9.

Wajant, H. (2003). "Targeting the FLICE Inhibitory Protein (FLIP) in cancer therapy." Mol Interv 3(3): 124-7.

Wilson, T. R., M. McEwan, et al. (2009). "Combined inhibition of FLIP and XIAP induces Bax-independent apoptosis in type II colorectal cancer cells." Oncogene 28(1): 63-72. 
Figures

FIGURE 1.

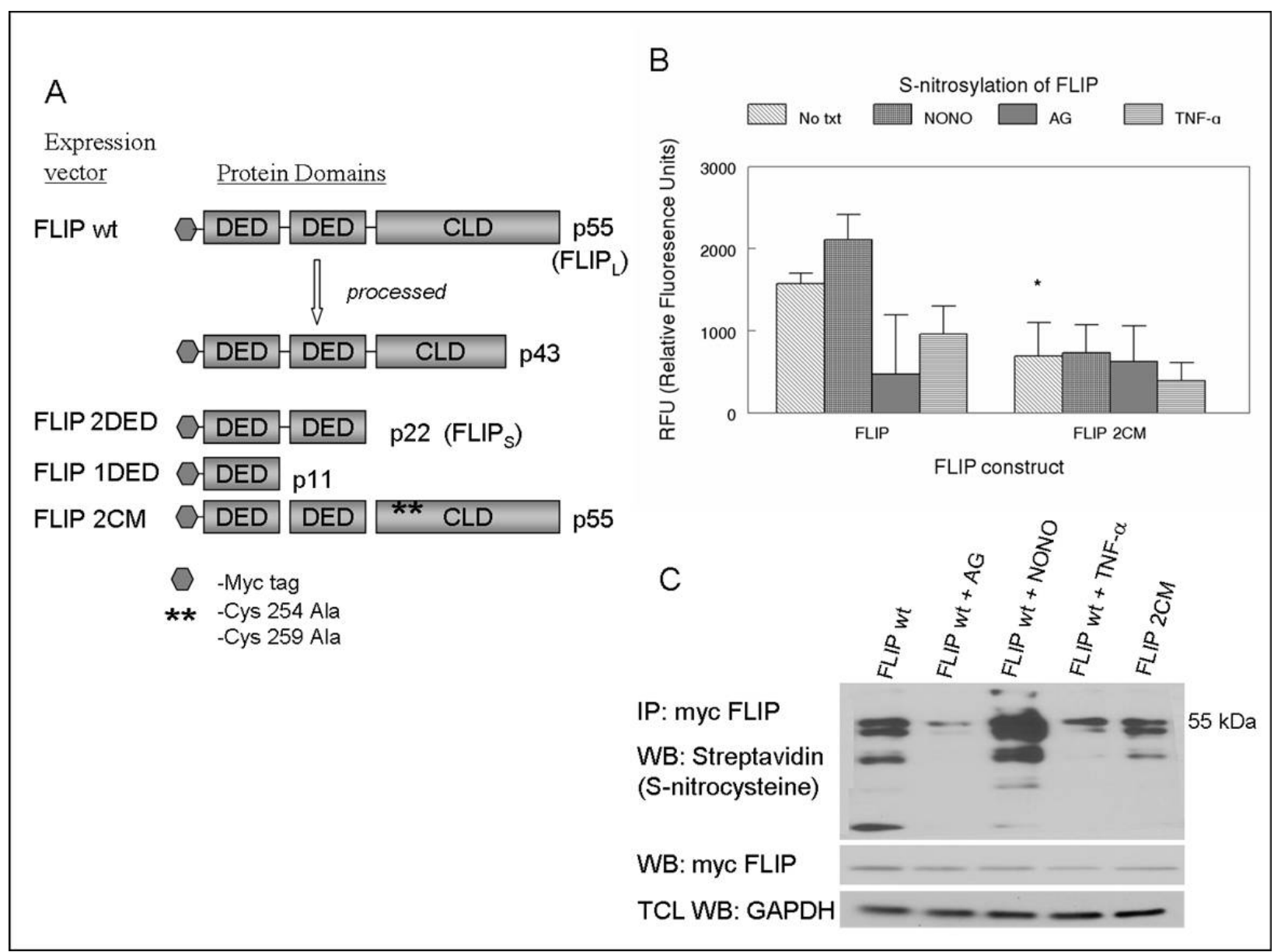

FIGURE 1. S-nitrosylation of FLIP A) A schematic of the domain structure of FLIP isoforms and expression vectors is shown. FLIP wt, FLIP 2DED (Death Effector Domains), FLIP 2CM. B) HEK-293 cells were transiently transfected with myc-FLIP wt or myc-FLIP $2 \mathrm{CM}$ and treated with media alone (no txt), $300 \mu \mathrm{M}$ AG, $400 \mu \mathrm{M}$ NONOate for 12 hours or $50 \mathrm{ng} / \mathrm{mLTNF}-\alpha$ for $30 \mathrm{~min}$. Analysis for S-nitrosylation was determined by fluorescence described in Materials and 
Methods. C) HEK-293 cells were transiently transfected with myc-FLIP or mycFLIP 2CM. myc-FLIP expressing cells were treated as in B). Lysates were coimmunoprecipitated using anti-myc antibodies and assayed for S-nitrocysteine by streptavidin using an S-nitrosylation kit. Membranes were stripped and reprobed with anti-myc antibodies to detect FLIP. Total cell lysates (TCL) were also probed with anti-GAPDH antibodies to control for loading. Error bars represent standard deviation. * indicates a statistical significance from no treatment (no txt) control $(p<0.05)$. Experiments were repeated three times. 
FIGURE 2.

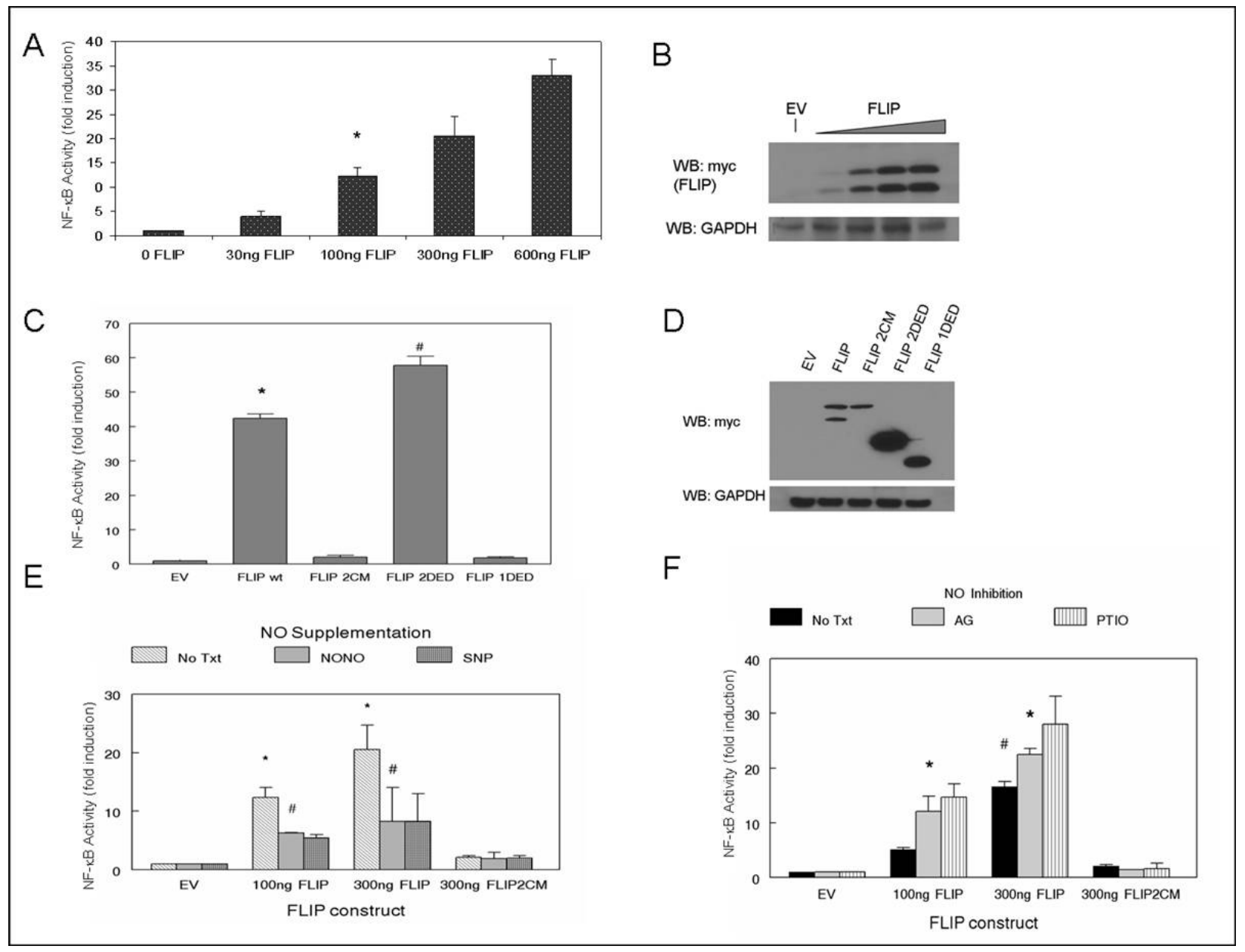

FIGURE 2. FLIP-mediated NF-kB activation HEK-293 cells were transiently transfected with an NF-kB-luciferase and Renilla-luciferase reporter and expression constructs for the FLIP domains, as indicated. Luciferase activity was determined by the dual-luciferase reporter assay. A) Cells were transiently transfected with increasing amounts of FLIP and an NF-kB luciferase reporter plasmid. NF-kB activity was determined by luminescence according to Materials and Methods. B) Total cell lysates were also analyzed by Western blot using an anti-myc antibody to verify the increasing expression levels of FLIP. C) Cells 
were transiently transfected with empty vector (EV), FLIP, FLIP 2CM, FLIP 2DED or FLIP 1DED and analyzed for NF-kB activity as noted in (A). D) Total cell lysates were also analyzed by Western blotting as above to verify expression of all constructs. E) Cells were transiently transfected with EV, FLIP, FLIP 2CM or FLIP 2DED. Cells treated with media alone, $400 \mu \mathrm{M}$ NONOate or $500 \mu \mathrm{M}$ SNP for 12 hours to supplement nitric oxide and analyzed for NF-KB activity as noted in (A). F) Cells were transiently transfected with empty vector (EV), FLIP wt or FLIP 2CM, and cells were treated with media alone, $300 \mu \mathrm{M}$ AG or $300 \mu \mathrm{M}$ PTIO for 12 hours to inhibit NO production. Lysates were analyzed for NF-kB activity as noted in (A). Error bars represent standard deviation. All luciferase experiments were repeated at least three times. ${ }^{*}$ indicates statistical significance from EV transfected cells. \# indicates statistical significance from no treatment (no txt) control $(p<0.05)$. 
FIGURE 3.

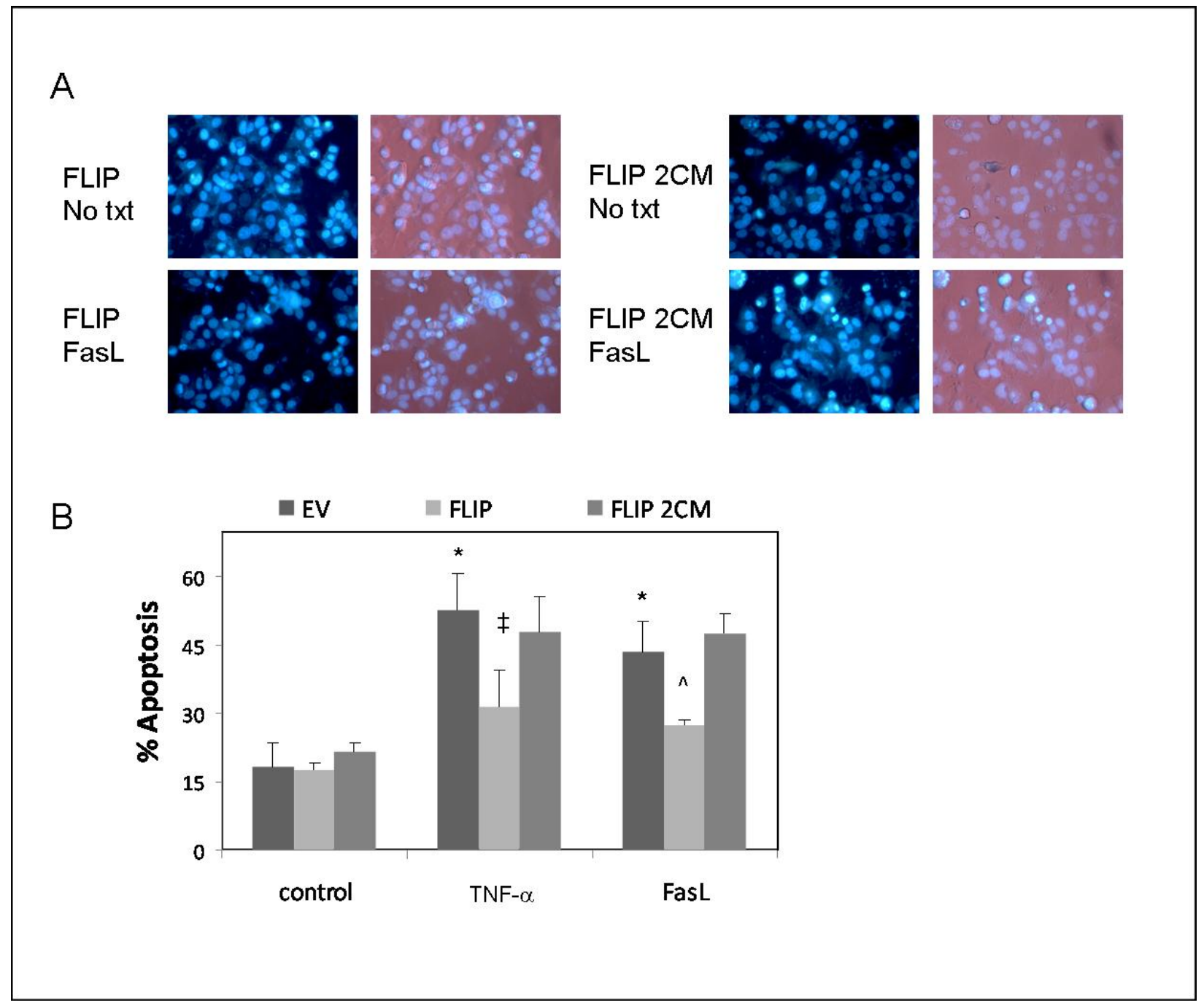

FIGURE 3. S-nitrosylation of FLIP modulates its anti-apoptotic activity.

MCF-7 cells were transiently transfected with FLIP or FLIP 2CM expression constructs. 24 hours post-transfection, cells were treated with media alone, 50 $\mathrm{ng} / \mathrm{mL}$ TNF- $\alpha$ or $200 \mathrm{ng} / \mathrm{mL}$ FasL for 16 hours. Cells were assayed by Hoechst 33342 staining to determine the percentage of apoptotic cells. A) Examples of Hoechst staining. B) Graphical representation of 15 fields of view analyzed. * 
indicates statistical significance from control and $\ddagger$ and ${ }^{\wedge}$ indicate statistical significance compared to treated EV $(p<0.05)$. 
FIGURE 4.

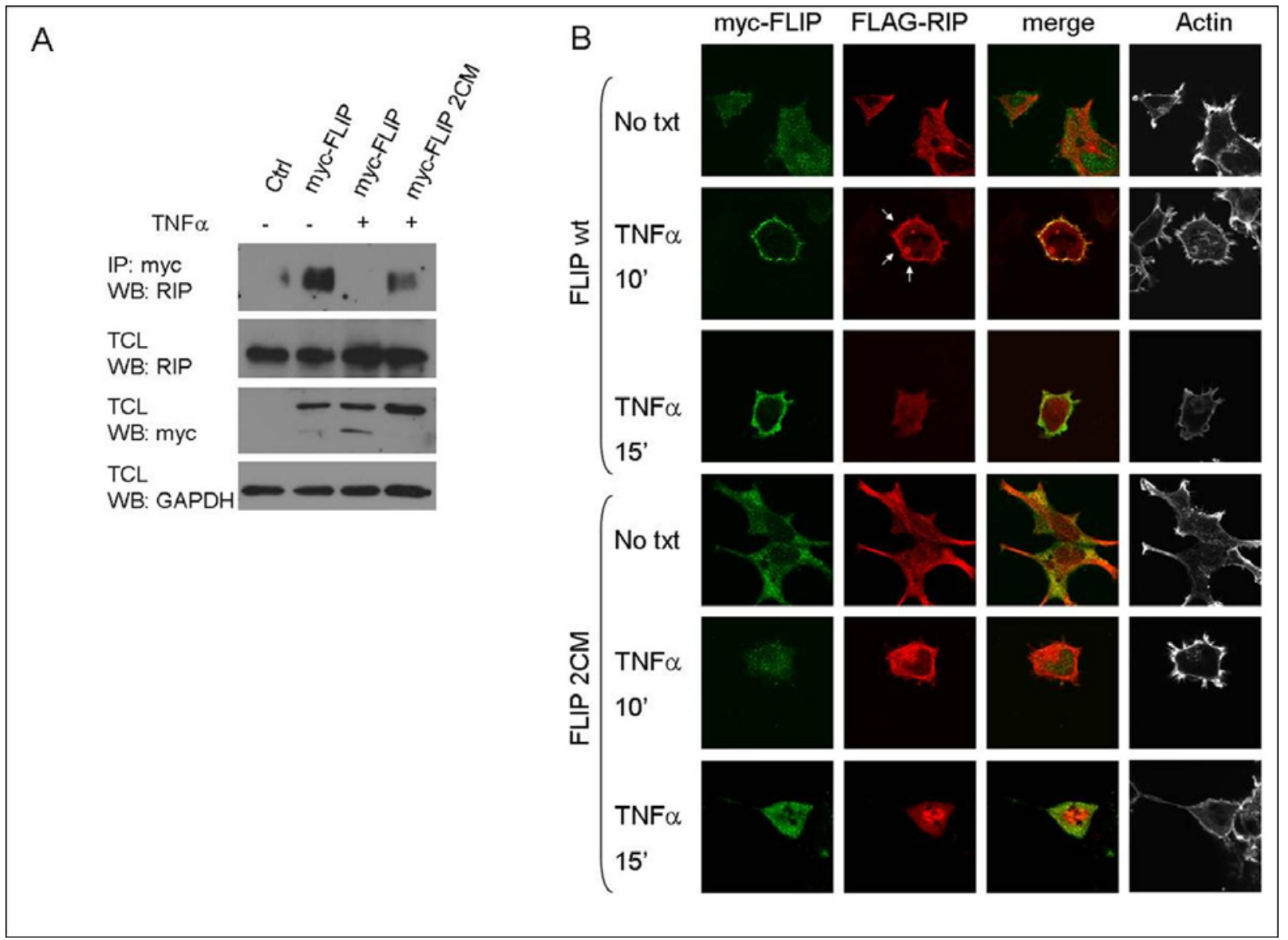

FIGURE 4. S-nitrosylation of FLIP modulates its interaction with RIP1 and localization of RIP. A) HEK-293 cells were transiently transfected with myc-FLIP or myc-FLIP 2CM expression constructs. 36 hours post-transfection, cells were either left untreated or treated with $50 \mathrm{ng} / \mathrm{mL}$ TNF- $\alpha$ for $30 \mathrm{~min}$. Lysates were coimmunoprecipitated with anti-myc antibodies to purify FLIP and probed for endogenous RIP. Membranes were stripped and reprobed with anti-myc antibodies. Lysates were also probed with anti-myc antibodies to detect FLIP, 
anti-RIP1 and anti-GAPDH antibodies. B) HEK-293 cells were transiently transfected with myc-FLIP, myc-FLIP 2CM and FLAG-RIP1 expression constructs as indicated, left untreated or stimulated with $50 \mathrm{ng} / \mathrm{mL}$ TNF- $\alpha$ for 10 or 15 min. Cells were fixed, immunostained with anti-myc and anti-FLAG antibodies and fluorescently labeled secondary antibodies and phalloidin to visualize actin and analyzed by confocal microscopy. White arrows indicate RIP localization to the membrane. 
FIGURE 5.

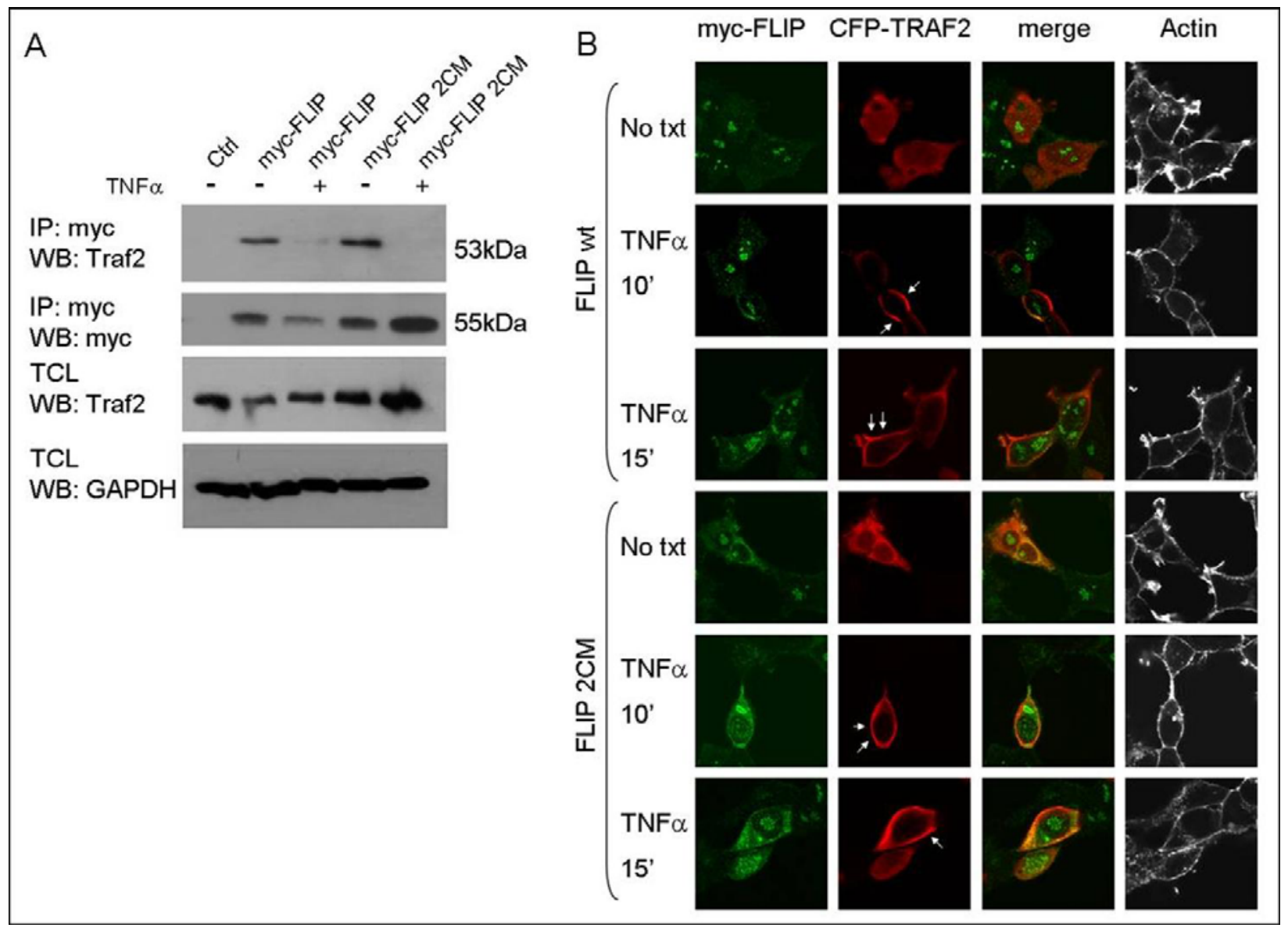

FIGURE 5. TRAF2 binding to FLIP is not dependent on FLIP S-nitrosylation

A) HEK-293 cells were transiently transfected with myc-FLIP or myc-FLIP 2CM expression constructs. 36 hours post-transfection, cells were left untreated or treated with $50 \mathrm{ng} / \mathrm{mL}$ TNF- $\alpha$ for $30 \mathrm{~min}$. Lysates were coimmunoprecipitated with anti-myc antibodies to purify FLIP and probed for endogenous TRAF2. Membranes were stripped and reprobed with anti-myc antibodies to detect FLIP. Total cell lysates (TCL) were also probed for myc-FLIP, TRAF2 and GAPDH to demonstrate equal loading. B) HEK-293 cells were transiently transfected with 
myc-FLIP, myc-FLIP 2CM and CFP-TRAF2 expression constructs as indicated, left untreated or stimulated with $50 \mathrm{ng} / \mathrm{mL}$ TNF- $\alpha$ for 10 or $15 \mathrm{~min}$. Cells were fixed, immunostained with anti-myc and anti-CFP antibodies and fluorescently labeled secondary antibodies and phalloidin to visualize actin and analyzed by confocal microscopy. White arrows indicate TRAF2 localization to the membrane. 
FIGURE 6.

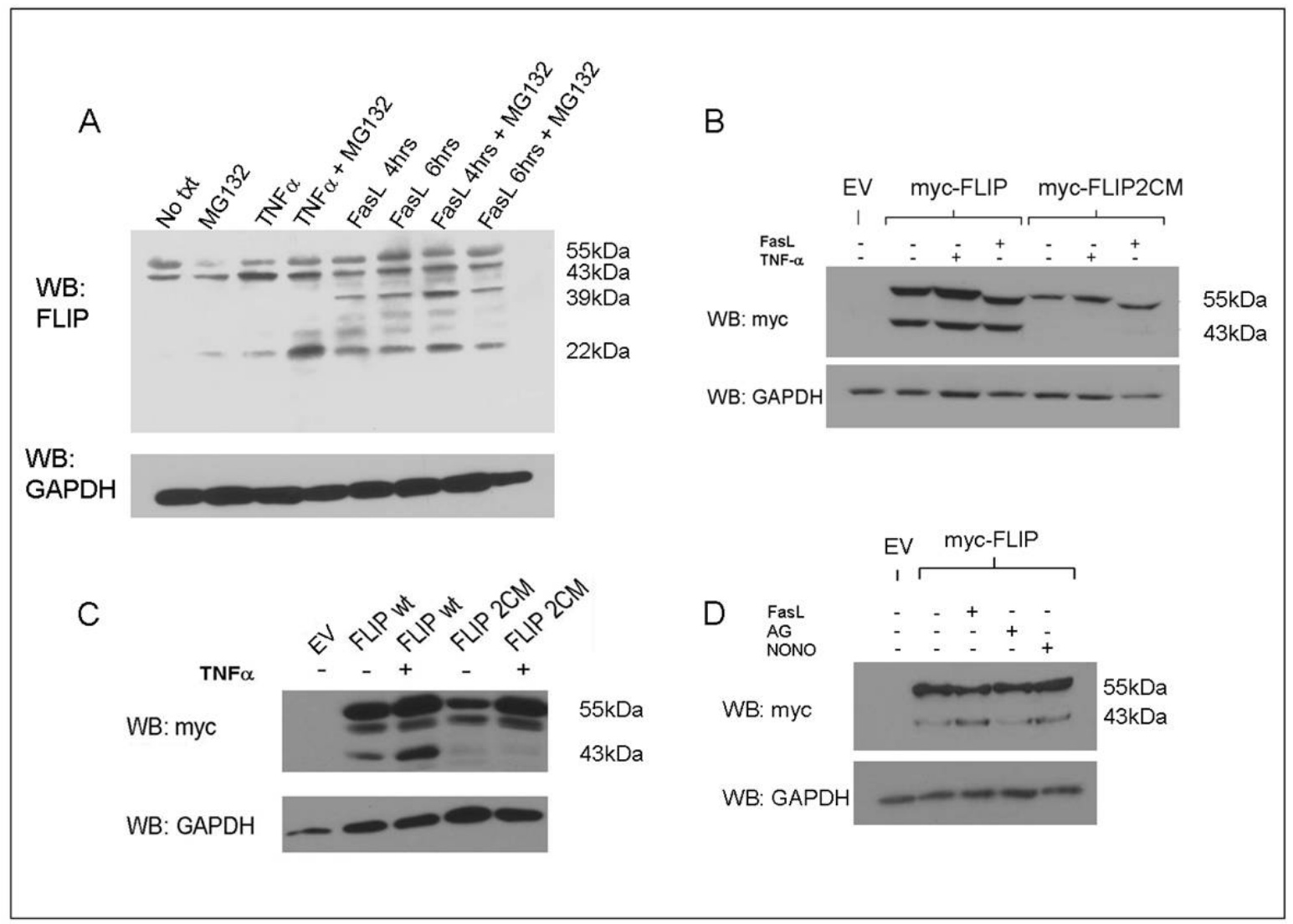

FIGURE 6. S-nitrosylation of FLIP promotes protein processing into shorter forms A) A549 cells were analyzed for endogenous FLIP expression. Cells were treated with media alone (txt), pretreated with $1 \mu \mathrm{M}$ MG132 for 24 hours, 50 $\mathrm{ng} / \mathrm{mL}$ TNF- $\alpha$ for 30 min or $100 \mathrm{ng} / \mathrm{mL}$ FasL for 4 and 6 hours. B) HEK-293 cells were transiently transfected with empty vector (EV), myc-FLIP or myc-FLIP 2CM expression constructs. 36 hours post-transfection, cells were left untreated or treated with $50 \mathrm{ng} / \mathrm{mL}$ TNF- $\alpha$ for $30 \mathrm{~min}$ or $200 \mathrm{ng} / \mathrm{mL}$ FasL for 4 hours and analyzed by Western blot using anti-myc antibodies to detect FLIP. Membranes 
were stripped and reprobed with anti-GAPDH antibodies to control for equal loading. C) MCF-7 cells were transiently transfected as in B, and left untreated or treated with $50 \mathrm{ng} / \mathrm{mL} \mathrm{TNF}-\alpha$ for $30 \mathrm{~min}$. Total lysates were analyzed by Western blot with anti-myc and anti-GAPDH antibodies. D) MCF-7 cells were transiently transfected with EV or myc-FLIP. 36 hours post-transfection, cells were left untreated or treated with $200 \mathrm{ng} / \mathrm{mL}$ FasL, $600 \mu \mathrm{M}$ AG or $400 \mu \mathrm{M}$ NONOate for 4 hours. Total lysates were analyzed by Western blot with anti-myc and anti-GAPDH antibodies. 


\title{
Chapter 3. Study 2: Regulation of Lung Cancer Cell Migration and Invasion by Reactive Oxygen Species and Caveolin-1
}

\author{
Sudjit Luanpitpong ${ }^{\ddagger \#}$, Siera Jo Talbott ${ }^{\dagger \#}$, Yon Rojanasakul ${ }^{\S}$, Ubonthip \\ Nimmannit", Varisa Pongrakhananon ${ }^{\ddagger}$, Liying Wang", and Pithi Chanvorachote ${ }^{+}$ \\ ${ }^{\ddagger}$ Pharmaceutical Technology (International) Program and ${ }^{+}$Department of \\ Pharmacology and Physiology, Chulalongkorn University, Bangkok 10330, \\ Thailand \\ ${ }^{\dagger}$ Cancer Cell Biology Program and ${ }^{\S}$ Department of Pharmaceutical Sciences, \\ West Virginia University, Morgantown, WV 26506, USA \\ "National Nanotechnology Center, Pathumthani 12120, Thailand \\ "National Institute for Occupational Safety and Health, Morgantown, WV 26505, \\ USA \\ \# These two authors contributed equally
}

\section{Published in Journal of Biological Chemistry, December 10 2010, v285 issue 50 , pages $388832-388840$.}

Corresponding Author:

Yon Rojanasakul

West Virginia University

Health Sciences Center

Morgantown, WV 26506

Tel: 3042931476

Fax: 3042932576

Email: yrojan@hsc.wvu.edu

Keywords: reactive oxygen species, migration; invasion; caveolin-1; cancer Abbreviations: ROS, reactive oxygen species; Cav-1, caveolin-1; NSCLC, nonsmall cell lung cancer; 


\section{Abstract}

Acquired capability of tumor cells to migrate and invade neighboring tissues is associated with high metastatic potential and advanced stage of cancers. Recently, signaling molecules such as reactive oxygen species (ROS) and caveolin-1 (Cav-1) have been implicated in the aggressive behavior of cancer cells. However, the role of specific ROS in cancer cell migration and Cav1 regulation are unclear. We demonstrate here that Cav-1 plays an important role in the migration and invasion of human lung carcinoma $\mathrm{H} 460$ cells and that these effects are differentially regulated by cellular ROS. Using various known inhibitors and donors of ROS, we found that different ROS have different effects on Cav-1 expression and cell migration and invasion. Superoxide anion and hydrogen peroxide down-regulated Cav-1 expression and inhibited cell migration and invasion, while hydroxyl radical up-regulated the Cav-1 expression and promoted cell migration and invasion. The down-regulating effect of superoxide anion and hydrogen peroxide on Cav-1 is mediated through a transcriptionindependent mechanism that involves protein degradation via the ubiquitinproteasome pathway. These results indicate the essential role of different ROS in cancer cell motility and through Cav-1 expression, which may provide a key mechanism controlling tumor progression and metastasis. The up-regulation of Cav-1 and cell motility by hydroxyl free radical suggests an important role of this ROS as a positive regulator of tumor progression. 


\section{Introduction}

Cancer cell migration and invasion are initial steps in metastasis which is a primary cause of cancer-related death. During metastasis, primary tumor cells migrate and invade neighboring tissues and enter the circulation to establish new or secondary tumor sites (Friedl and Wolf 2003; Gupta and Massague 2006; Bacac and Stamenkovic 2008). Increasing evidence suggests that signaling molecules presenting in the tumor microenvironment have a significant impact on the migratory properties of cancer cells (Laurent, Nicco et al. 2005; Kopfstein and Christofori 2006). For example, increased ROS in the tumor micro-environment has been associated with increased aggressiveness of cancer cells (Droge 2002). While several studies have investigated the effects of ROS on cell migration and invasion, variable results have been reported depending on the type of ROS, dose, and production site, as well as the tissue type of cells (O'Toole, Goel et al. 1996; Urbich, Dernbach et al. 2002; Novo, Marra et al. 2006; Kumar, Koul et al. 2008). Several mechanisms of ROS regulation of cancer cell migration have been proposed; most of which involve alterations of cellular cytoskeleton and adhesion molecules. For instance, ROS have been reported to regulate integrin (Chiarugi, Pani et al. 2003; Svineng, Ravuri et al. 2008), small GTPase Rho family proteins (Alexandrova, Kopnin et al. 2006; Tobar, Caceres et al. 2008), focal-contact forming proteins (Chiarugi, Pani et al. 2003), and extracellular matrix-degrading enzymes such as matrix metallo proteinases (MMPs) (Nelson and Melendez 2004; Lee, Hwang et al. 2008; Svineng, Ravuri et al. 2008). 
Caveolin-1 (Cav-1) is an essential structural protein component of the plasma membrane micro domains called caveolae. It has been shown to function in vesicular trafficking, signal transduction, and tumor progression. Cav1 interacts with several signaling molecules including Ha-Ras, Src-family tyrosine kinases, G protein a subunits, and protein kinase C (Oka, Yamamoto et al. 1997; Grande-Garcia, Echarri et al. 2007; Joshi, Strugnell et al. 2008; Park and Han 2009). Increased expression of Cav-1 has been observed in lung adenocarcinoma and prostate cancer, which are associated with their invasiveness (Ho, Huang et al. 2002; Yoo, Park et al. 2003; Williams, Hassan et al. 2005). In lung carcinoma, the elevated Cav-1 expression is also associated with an increase in metastatic capacity and poor survival of patients (Yoo, Park et al. 2003; Moon, Lee et al. 2005; Cassoni, Daniele et al. 2009). On the other hand, Cav-1 has been shown to have a suppressive effect on pancreatic and breast cancer cell motility (Payne, Bonnefous et al.; Fiucci, Ravid et al. 2002; Sloan, Stanley et al. 2004). Thus, the role of Cav-1 in cancer cell migration and metastasis remains unclear and appears to be cell type dependent.

Increased oxidative stress and ROS production have also been associated with many human metastatic tumors including the lung (Chung-man Ho, Zheng et al. 2001; Misthos, Katsaragakis et al. 2005), breast, prostate, colon (Szatrowski and Nathan 1991), and ovary (Hileman, Liu et al. 2004). ROS such as superoxide anion $\left(\mathrm{O}_{2}{ }^{-}\right)$, hydrogen peroxide $\left(\mathrm{H}_{2} \mathrm{O}_{2}\right)$, and hydroxyl radical $(\mathrm{OH} \cdot)$ have been shown to be upregulated in the tumor micro-environment and have been suggested to play a role in tumor progression and metastasis (Laurent, 
Nicco et al. 2005; Storz 2005). While ROS are likely to affect cancer cell motility and invasiveness through multiple mechanisms, Cav-1 may play a key role in this process since Cav-1 is known to be abnormally regulated in invasive tumors and play a role in cancer cell migration (Ho, Huang et al. 2002; Yoo, Park et al. 2003; Moon, Lee et al. 2005; Williams, Hassan et al. 2005; Cassoni, Daniele et al. 2009). Cav-1 is also subject to regulation by ROS (Volonte, Zhang et al. 2002; Esme, Cemek et al. 2008), but the underlying mechanism of regulation and the specific ROS involved are unclear. In this study, we investigated the roles of specific ROS including $\mathrm{O}_{2}{ }^{-}, \mathrm{H}_{2} \mathrm{O}_{2}$, and $\mathrm{OH}$. in Cav-1 expression and cell migration, and determined the mechanisms of regulation in human lung carcinoma H460 cells. The following specific questions are addressed: a) whether Cav-1 plays a role in the cell migration and invasion; b) whether Cav-1 is regulated by ROS and if so by what mechanism; and c) what specific ROS are involved and how they affect cell migration and invasion. 


\section{Experimental Procedures}

Cells and Reagents: Human lung cancer epithelial $\mathrm{H} 460$ cells were obtained from American Type Culture Collection (ATCC, Manassas, VA). The cells were cultured in RPMI 1640 medium containing 10\% fetal bovine serum (FBS), 2 mM L-glutamine, 100 units $/ \mathrm{ml}$ penicillin, and $100 \mu \mathrm{g} / \mathrm{ml}$ streptomycin in a $5 \% \mathrm{CO}_{2}$ environment at $37^{\circ}$ C. 3-(4,5-Dimethyl-thiazol-2-yl)-2,5-diphenyl tetrazolium bromide (MTT), 2', 7'-dichlorodihydrofluorescein diacetate ( $\mathrm{H}_{2}$ DCF-DA), 2,3dimethoxy-1,4-naphthoquinone (DMNQ), 5,5-dimethyl-1-pyrroline-N-oxide (DMPO), Hoechst 33342, hydrogen peroxide, catalase, ferrous sulfate heptahydrate, sodium formate, deferoxamine, lactacystin, type IV collagen, and laminin were obtained from Sigma (St. Louis, MO). Mn(III)tetrakis(4-benzoic acid)porphyrin chloride (MnTBAP) and concanamycin A were obtained from Calbiochem (San Diego, CA). Antibodies for Cav-1, ubiquitin, $\beta$-actin, peroxidase-labeled secondary antibodies, and protein-G agarose were obtained from Abcam Inc. (Cambridge, MA). Antibody for phospho-Akt (Ser-473) was from Cell Signaling Technology, Inc. (Beverly, MA).

Generation of Stable Cav-1 Overexpressing Cells: H460 cells were seeded in 6well plate until they reached $60 \%$ confluence. The cells were then transfected with caveolin-1 plasmid (pEX_Cav-1-YFP) (ATCC) or control plasmid (pcDNA3) using Lipofectamine reagent (Invitrogen, Carlsbad, CA) in culture medium in the absence of serum. After 12 hours of incubation, the medium was replaced with complete culture medium containing $10 \%$ FBS. Approximately 36 hours after the 
beginning of the transfection, the cells were trypsinized and plated onto $75-\mathrm{ml}$ culture flasks, and cultured for 28 days with neomycin containing medium (800 $\mu \mathrm{g} / \mathrm{ml}$ ). The pooled stable transfectant was identified by Western blotting of Cav1 and was cultured in neomycin-free RPMI medium for at least two passages before each experiment.

Inhibition of Cav-1 by RNA Interference: Lentiviral transduction particles carrying short hairpin RNA (shRNA) sequence against human Cav-1 (5'-CCGGGACGTG GTCAAGATTGACTTTCTCGAGAAAGTCAATCTTGACCACGTCTTTTT-3') and control non-target sequence (5'-CCGGCAACAAGATGAAGAGCACCAACTCGA GTTGGTGCTCTTCATCTTGTTGTTTTT-3') were used to knockdown Cav-1 expression in $\mathrm{H} 460$ cells. The viral vectors were obtained commercially from Sigma Chemical Inc. (Cat \# NM_001753 and SHC002V, respectively) and were used according to the manufacturer's instruction. Briefly, cells were seeded in 6well plates ( $5 \times 10^{5} /$ well) and incubated with Cav-1 shRNA lentiviral particles or control particles at the multiplicity of infection (MOI) of 1.5 in the presence of hexadimethrine bromide $(8 \mu \mathrm{g} / \mathrm{ml})$ for 36 hours. Transfected cells were analyzed for Cav-1 by Western blotting prior to use.

Cytotoxicity Assay: Cell viability was determined by MTT assay. After specific treatments, cells in 96-well plate were incubated with $500 \mu \mathrm{g} / \mathrm{ml}$ of MTT for 4 hours at $37^{\circ} \mathrm{C}$. The intensity of formazan product was measured at $550 \mathrm{~nm}$ 
using a microplate reader. Absorbance ratio of treated to non-treated control cells was calculated and presented as relative cell viability.

ROS Detection: Cellular ROS was determined by flow cytometry using $\mathrm{H}_{2} \mathrm{DCF}$ DA as a fluorescent probe and by electron spin resonance (ESR) spectroscopy using DMPO as a spin trapping agent. For flow cytometric measurements, cells were incubated with $\mathrm{H}_{2} \mathrm{DCF}-\mathrm{DA}(10 \mu \mathrm{M})$ for 30 minutes at $37^{\circ} \mathrm{C}$, after which they were washed and resuspended in phosphate-buffered saline (PBS) and analyzed for fluorescence intensity using a $485-\mathrm{nm}$ excitation beam and a 538-nm bandpass filter (FACSort, Becton Dickinson, Rutherford, NJ). The mean fluorescence intensity was quantified by CellQuest software (Becton Dickinson) analysis of the recorded histograms. For ESR measurements, cells were incubated with DMPO (100 $\mathrm{mM}$ ) for 10 minutes at $37^{\circ} \mathrm{C}$ in the presence or absence of ROS modulators. ESR signals were measured using a Bruker EMX spectrometer (Bruker Instruments, Billerica, MA) and a flat cell assembly. Hyperfine couplings were measured (to $0.1 \mathrm{G}$ ) directly from magnetic field separation using potassium tetraperoxo-chromate and 1,1-diphenyl-2-picrylhydrazyl as reference standards. An Acquisit program (Bruker Instruments) was used for data acquisition and analysis.

Migration Assay: Cell migration was determined by wound or scratch assay. Monolayer of cells was cultured in 24-well plate, and then a wound space was made with $1 \mathrm{~mm}$ width tip. After rinsing with PBS, the cell monolayers were 
treated with specific ROS modulators and allowed to migrate for 24 hours. Micrographs were taken under a phase-contrast microscope (100X) (Olympus DP70, Melville, NY) and wound spaces were measured from ten random fields of view using an Olympus DP controller software. Quantitative analysis of cell migration was performed by using an average wound space from those random fields of view, and the percentage of change in the wound space was calculated using the formula: $\%$ change $=$ (average space at time 0 hours $)-($ average space at time 24 hours) / (average space at time 0 hours $) \times 100$. Relative cell migration was calculated by dividing the percentage change in the wound space of treated cells by that of the control cells in each experiment.

Invasion Assay: Invasion assay was performed using a 24-well Transwell unit with polycarbonate (PVDF) filters ( $8 \mu \mathrm{m}$ pore size). The membrane was coated with a mixture of laminin $(50 \mu \mathrm{g} / \mathrm{ml})$, type IV collagen $(50 \mu \mathrm{g} / \mathrm{ml})$, and gelatin solution ( $2 \mathrm{mg} / \mathrm{ml}$ in $10 \mathrm{mM}$ glacial acetic acid). Cells at the density of $2 \times 10^{4}$ cells per well were seeded into the upper chamber of the Transwell unit in serumfree medium. The lower chamber of the unit was added with a medium containing 10\% FBS. After incubation with specific test agents for 24 hours at $37^{\circ} \mathrm{C}$, the medium in the upper chamber was sucked out and the cells on the upper side of membrane were removed with a cotton swab. Cells that invaded to the underside of the membrane were stained with $10 \mu \mathrm{g} / \mathrm{ml} \mathrm{Hoechst} 33342$ for 10 minutes and visualized and scored under a fluorescence microscope. 
Western Blot Analysis: After specific treatments, cells were incubated in lysis buffer containing $20 \mathrm{mM}$ Tris-HCl (pH 7.5), 1\% Triton X-100, $150 \mathrm{mM}$ sodium chloride, $10 \%$ glycerol, $1 \mathrm{mM}$ sodium orthovanadate, $50 \mathrm{mM}$ sodium fluoride, 100 $\mathrm{mM}$ phenylmethylsulfonyl fluoride, and a commercial protease inhibitor mixture (Roche Molecular Biochemicals) at $4^{\circ} \mathrm{C}$ for 20 minutes. Cell lysates were collected and determined for protein content using the Bradford method (Bio-Rad Laboratories, Hercules, CA). Proteins $(40 \mu \mathrm{g})$ were resolved under denaturing conditions by $10 \%$ sodium dodecyl sulfate-polyacrylamide gel electrophoresis (SDS-PAGE) and transferred onto a nitrocellulose membrane. The membranes were blocked for 1 hour in $5 \%$ nonfat dry milk in TBST (25 mM Tris-HCl, $\mathrm{pH} 7.4$, $125 \mathrm{mM}$ sodium chloride, $0.05 \%$ Tween 20 ) and incubated with appropriate primary antibodies at $4^{\circ} \mathrm{C}$ for 1 hour. Membranes were washed twice with TBST for 10 minutes and incubated with horseradish peroxidase-labeled isotypespecific secondary antibodies for 1 hour at room temperature. The immune complexes were then detected by enhanced chemiluminescence detection system (Amersham Biosciences, Piscataway, NJ) and quantified using analyst/PC densitometry software (Bio-Rad Laboratories, Hercules, CA).

Immunoprecipitation: After specific treatments, cells were washed with PBS and lysed in lysis buffer at $4^{\circ} \mathrm{C}$ for 20 minutes. Cell lysates were collected and determined for protein content using the Bradford method. Lysate proteins (60 $\mu g$ ) were incubated with Cav- 1 antibody for 14 hours at $4^{\circ} \mathrm{C}$, followed by a 4 hour incubation with protein G-conjugated agarose at $4^{\circ} \mathrm{C}$. The immune 
complexes were washed 6 times with cold lysis buffer and resuspended in $2 \mathrm{X}$ Laemmli sample buffer. The immune complexes were separated by $10 \%$ SDSPAGE and analyzed by Western blotting as described.

Quantitative Real-Time PCR: Total RNA was extracted with Trizol reagent (Invitrogen). One microgram of extracted RNA was reverse-transcribed in a 100$\mu l$ reaction mixture containing $500 \mu \mathrm{M}$ dNTP, 125 units of MultiScribe Reverse Transcriptase (Applied Biosystems, Foster City, CA), 40 units of RNase inhibitor, $2.5 \mu \mathrm{M}$ oligo(dT), $1 \mathrm{X}$ Taq-Man reverse transcriptase buffer, and $5 \mathrm{mM} \mathrm{MgCl}_{2}$ at $48{ }^{\circ} \mathrm{C}$ for 40 minutes. The primers used in this study were designed using Primer Express software (Applied Biosystems): Cav-1 (\#Al878826) forward 5'-CGAGAA GCAAGTGTACGACGC-3', and reverse 5'-ACCACGTCATCGTTGAGGTG-3'; glyceralde-hyde-3-phosphate dehydrogenase (GAPDH) forward, 5'-GAAGGTGA AGGTCGGAGTC-3', and reverse 5'-GAAGATGGTGATGGGATTTC-3'. Amplification was performed at the following cycling conditions: $95^{\circ} \mathrm{C}$ for 10 minutes followed by 40 cycles at $95^{\circ} \mathrm{C}$ for 15 seconds and $60{ }^{\circ} \mathrm{C}$ for 1 minute. A SYBR Green PCR Master Mix (Applied Biosystems) was used with $1 \mathrm{ng}$ of cDNA and with 100-400 nM primers. A negative control without any cDNA template was run with every assay. All PCR reactions were performed by using $A B I$ PRISM 7900 Sequence Detection System (Applied Biosystems). Relative mRNA levels were determined by using the comparative CT (threshold cycle) method (40), where the caveolin-1 target is normalized to the control and compared with a reference sample (assigned a relative value of 1 ) by the equation: $2^{-\Delta \Delta C T}$. 
Statistical Analysis: Data were represented as the means \pm S.D. from three or more independent experiments. Statistical analysis was performed by Student's $t$ test at a significance level of $p<0.05$. 


\section{Results}

\section{Differential Effect of ROS on Cell Migration}

ROS have been shown to be involved in several cellular migratory processes including wound repair, metastasis, and angiogenesis (O'Toole, Goel et al. 1996; Steiling, Munz et al. 1999; Urbich, Dernbach et al. 2002; Ushio-Fukai and Alexander 2004; Novo, Marra et al. 2006; Kumar, Koul et al. 2008). However, the role of specific ROS and regulatory mechanisms are not well understood. We tested whether ROS play a role in the migration of lung carcinoma H460 cells and determined the specific ROS involved. Cells were treated with various known inducers and scavengers of ROS, and their effect on cell migration was determined by wound migration assay. Fig. $1 \mathrm{~A}$ shows that treatment of the cells with MnTBAP, a superoxide dismutase (SOD) mimetic and scavenger of $\mathrm{O}_{2}^{-}$, stimulated the migration of cells across the wound space, whereas treatment of the cells with DMNQ, a known inducer of $\mathrm{O}_{2}^{-}$(Ishihara, Shiba et al. 2006; Herr, Zhou et al. 2007), had an opposite effect. Likewise, treatment of the cells with catalase (CAT) $\left(\mathrm{H}_{2} \mathrm{O}_{2}\right.$ scavenger) promoted cell migration, whereas treatment with $\mathrm{H}_{2} \mathrm{O}_{2}$ inhibited the migration. These results indicate the inhibitory role of $\mathrm{O}_{2}{ }^{-}$and $\mathrm{H}_{2} \mathrm{O}_{2}$ in the migration of $\mathrm{H} 460$ cells during wound healing. In contrast to the above findings, treatment of the cells with sodium formate (NaFM), a known OH. scavenger (Dokka, Shi et al. 2001; Wang, Leonard et al. 2004), inhibited the migration, while the $\mathrm{OH} \cdot$ generator ferrous sulfate $\left(\mathrm{FeSO}_{4}\right)$ promoted this effect. These results suggest the differential roles 
of ROS in the regulation of cancer cell migration with $\mathrm{OH}$. playing a promoting role and $\mathrm{O}_{2}{ }^{-}$and $\mathrm{H}_{2} \mathrm{O}_{2}$ having an inhibitory role.

To ensure that the observed inhibitory and stimulating effects of ROS modulators was not caused by a cytotoxic effect, cells were treated with the same concentrations of the test modulators and their effect on cell viability was determined by MTT assay. The results show that none of the treatments caused a significant effect on cell viability as compared to non-treated control (Fig. 1B).

\section{Detection of Cellular ROS}

To provide supporting evidence for the role of ROS in cell migration, cellular ROS in response to the above treatments were determined by flow cytometry and electron spin resonance (ESR). Flow cytometric analysis was performed using $\mathrm{H}_{2}$ DCF-DA as a fluorescent probe. $\mathrm{H}_{2}$ DCF-DA is a general oxidative probe that can detect multiple ROS. The probe enters the cells and is cleaved by cellular esterases to yield a non-fluorescent product, dichlorofluorescin $(\mathrm{DCFH})$, which is trapped inside the cells. Upon oxidation by ROS, DCFH is converted to the fluorescent product DCF which is detected by flow cytometry. Fig. $2 \mathrm{~A}$ shows that the ROS inducers $\mathrm{DMNQ}, \mathrm{H}_{2} \mathrm{O}_{2}$, and $\mathrm{FeSO}_{4}$ were able to increase the cellular DCF fluorescence intensity over control level, whereas the ROS scavengers MnTBAP, CAT, and NaFM decreased the fluorescence intensity, indicating multiple ROS generation and scavenging by the treatments. 
To confirm the formation of ROS in the treated cells, ESR measurements were performed using DMPO as a spin trapping agent to aid the detection of short-lived oxygen free radicals. Fig. 2B shows a typical ESR spectrum generated by incubating $\mathrm{H} 460$ cells in culture medium with DMPO. A weak ESR signal consisting of a 1:2:2:1 quartet which is a characteristic of DMPO-OH. adduct was observed, indicating the formation of $\mathrm{OH}$. radicals. Addition of the Fenton catalyst and $\mathrm{OH}$. generator $\mathrm{FeSO}_{4}$ to the cells intensified this signal, supporting the generation of $\mathrm{OH}$. radicals. Addition of the $\mathrm{OH}$. scavenger $\mathrm{NaFM}$ to the $\mathrm{FeSO}_{4}$-treated cells inhibited the ESR signal, indicating the specificity of $\mathrm{OH}$. detection and their scavenging by NaFM under the test conditions. Neither $\mathrm{H}_{2} \mathrm{O}_{2}$ nor $\mathrm{O}_{2}{ }^{-}$was detectable in this study since the former is not a free radical and the latter does not form a stable adduct with the spin trapping agent DMPO.

Caveolin 1 Promotes Cell Migration and Invasion

Cav-1 has been shown to modulate cell migration in different cell types (Oka, Yamamoto et al. 1997; Fiucci, Ravid et al. 2002; Ho, Huang et al. 2002; Williams, Hassan et al. 2005; Grande-Garcia, Echarri et al. 2007; Joshi, Strugnell et al. 2008; Park and Han 2009). We tested whether Cav-1 can regulate the migration and invasion of lung carcinoma $\mathrm{H} 460$ cells. The cells were stably transfected with Cav-1 or control plasmid, and their effects on Cav-1 expression, cell migration and invasion were determined. Western blot analysis of Cav-1 expression shows a substantial increase in the expression in Cav-1-transfected cells as compared to vector-transfected control (Fig. 3A). Wound migration 
assay shows that the Cav-1-transfected cells exhibited an increase in migratory activity as compared to control-transfected cells (Fig. 3B). Transwell invasion assay similarly indicates an increase in cell invasiveness of the Cav-1 overexpressing cells as compared to control-transfected cells (Fig. 3C).

To confirm the role of Cav-1 in cell migration and invasion, Cav-1 expression was inhibited by RNA interference using shRNA against Cav-1 (shCav-1). H460 cells were treated with shCav-1 viral particles or control shRNA (shCon) particles, and their effects on cell migration and invasion were determined. Fig. 3D shows that Cav-1 expression was substantially reduced in shCav-1-treated cells as compared to shCon-treated cells (left panel). Rescue experiment was performed on the shCav-1 knockdown cells to exclude the offtarget effect of shCav-1 (right panel). Figs. 3E and 3F show that shCav-1 cells exhibited reduced migratory and invasive activities as compared to shCon cells, and that over-expression of Cav-1 in these cells reversed the migratory and invasive activities. These results support the promoting role of Cav-1 in migration and invasion of $\mathrm{H} 460$ cells.

To determine whether the effects of ROS on cell migration and invasion are dependent on Cav-1 expression, shCav-1 and shCon cells were treated with DMNQ, $\mathrm{H}_{2} \mathrm{O}_{2}$, and $\mathrm{FeSO}_{4}$ and their effects on cell migration and invasion were determined. Figs. 4A and 4B show that $\mathrm{DMNQ}$ and $\mathrm{H}_{2} \mathrm{O}_{2}$ inhibited cell migration and invasion in shCon cells but not in shCav-1 cells. $\mathrm{FeSO}_{4}$ increased cell migration and invasion in shCon cells but had minimal effects in shCav-1 cells. 
These results indicate the role of Cav-1 in the ROS effects on cell migration and invasion.

Cav-1 has been shown to regulate cell migration through a PI3K/Aktdependent mechanism (Ravid, Maor et al. 2005; Li, Ren et al. 2009). We tested whether knockdown of Cav-1 affects Akt activity by analyzing phosphorylated Akt (pAkt) levels in shCav-1 and shCon cells. Fig. 4C shows that pAkt level was substantially reduced in shCav- 1 cells as compared to shCon cells. This result is consistent with our previous report showing the induction of Akt activation by Cav-1 overexpression (Chanvorachote, Nimmannit et al. 2009). We also investigated Akt activity in $\mathrm{H} 460$ cells in response to specific ROS. Fig. 4D shows that DMNQ and $\mathrm{H}_{2} \mathrm{O}_{2}$ caused a decrease in pAkt level, whereas $\mathrm{FeSO}_{4}$ promoted it. These results are consistent with the observed effects of specific ROS on Cav-1 expression and cell motility, supporting the role of Cav-1 in the migratory process through Akt signaling.

\section{ROS Regulate Cav-1 Expression}

Cav-1 may be regulated by ROS which may represent a key mechanism of cell migratory regulation by ROS. To test this possibility, cells were treated with various ROS modulators and their effect on Cav-1 expression was determined by Western blotting. Fig. 5A shows that treatment of the cells with DMNQ or $\mathrm{H}_{2} \mathrm{O}_{2}$ substantially down-regulated the expression of Cav-1, whereas treatment with $\mathrm{FeSO}_{4}$ up-regulated the expression. Consistent with the inhibitory role of $\mathrm{O}_{2}{ }^{-}$and $\mathrm{H}_{2} \mathrm{O}_{2}$, the scavengers of these ROS (MnTBAP and CAT 
respectively) promoted the Cav-1 expression (Fig. 5B). In contrast, the $\mathrm{OH}$. scavenger NaFM inhibited the expression, supporting the positive regulatory role of $\mathrm{OH}$. in Cav-1 expression. This latter result was confirmed by the observation that deferoxamine (DFX), a known metal chelator and inhibitor of $\mathrm{OH} \cdot$, also inhibited Cav-1 expression (Fig. 5B).

Role of Specific ROS in Cell Migration, Invasion, and Cav-1 Expression

To further determine the relationship between Cav-1 expression and cell motility regulation by different ROS, cells were treated with various concentrations of ROS modulators and their effects on cell migration, invasion, and Cav-1 expression were determined. Figs. $6 \mathrm{~A}$ and $6 \mathrm{D}$ show that $\mathrm{DMNQ}$ caused a dose-dependent and parallel decrease in both cell migration and Cav-1 expression. Addition of MnTBAP reversed both of these effects of DMNQ, indicating the role of $\mathrm{O}_{2}^{-}$in the processes and their association. Similar to DMNQ, $\mathrm{H}_{2} \mathrm{O}_{2}$ caused a dose-dependent and concomitant decrease in cell migration (Fig. 6B) and Cav-1 expression (Fig. 6E), both of which were inhibited by catalase. In contrast to $\mathrm{O}_{2}{ }^{-}$and $\mathrm{H}_{2} \mathrm{O}_{2}, \mathrm{OH}$. promoted cell migration (Fig. $6 \mathrm{C}$ ) and Cav-1 expression (Fig. 6F) as indicated by their positive responses to $\mathrm{FeSO}_{4}$ treatment and their inhibition by NaFM co-treatment, which were shown to induce and inhibit respectively the formation of $\mathrm{OH}$. radicals (Fig. 2B). Together, these results indicate the positive regulatory role of $\mathrm{OH} \cdot$ and the opposing role of $\mathrm{O}_{2}^{-}$ and $\mathrm{H}_{2} \mathrm{O}_{2}$ in cell migration through Cav-1 expression. A similar finding was observed with regard to the role of different ROS in cell invasion (Fig. 6G). 
Superoxide and Hydrogen Peroxide Downregulate Cav-1 by UbiquitinProteasomal Degradation

To investigate the mechanism of Cav-1 down-regulation by $\mathrm{O}_{2}{ }^{-}$and $\mathrm{H}_{2} \mathrm{O}_{2}$, cells were treated with DMNQ and $\mathrm{H}_{2} \mathrm{O}_{2}$, and analyzed for Cav-1 mRNA expression by quantitative real-time RT-PCR. Although causing a substantial decrease in Cav-1 protein expression (Fig. 6A), treatment of the cells with DMNQ and $\mathrm{H}_{2} \mathrm{O}_{2}$ did not cause a corresponding decrease in Cav-1 mRNA expression (Fig. 7A). In fact, a slight increase was observed, possibly due to stress induction. Thus, $\mathrm{O}_{2}{ }^{-}$and $\mathrm{H}_{2} \mathrm{O}_{2}$ appear to regulate Cav-1 protein expression through a transcription-independent mechanism.

Since many proteins are regulated by ROS through protein degradation, we tested the potential role of degradation in ROS-mediated Cav-1 downregulation. Degradation of endogenous proteins normally occurs through two major pathways, proteasomal and lysosomal. We therefore tested the effect of proteasome inhibitors, lactacystin and MG132, and lysosome inhibitor, concanamycin A, on ROS-induced Cav-1 down-regulation. Figs. 7B and 7C show that lactacystin and MG132, but not concanamycin A, inhibited Cav-1 down-regulation by $D M N Q$ and $\mathrm{H}_{2} \mathrm{O}_{2}$, suggesting proteasomal degradation as a primary mechanism of Cav-1 down-regulation by ROS.

Because proteins are targeted for proteasomal degradation via ubiquitination, we tested whether ROS modulators induce ubiquitination of Cav-1 protein. Cells were treated with $\mathrm{DMNQ}$ and $\mathrm{H}_{2} \mathrm{O}_{2}$, and cell lysates were prepared 
and immunoprecipitated by anti-Cav-1 antibody. The resulting immune complexes were then analyzed for ubiquitination by Western blots using antiubiquitin antibody. Figures 7D and 7E show that both DMNQ and $\mathrm{H}_{2} \mathrm{O}_{2}$ were able to induce ubiquitination of Cav-1 and that these effects were inhibited by cotreating the cells with MnTBAP and catalase, respectively. Together, these results indicate ROS-mediated down-regulation of Cav-1 through ubiquitinproteasomal degradation.

ROS Regulate Migration and Invasion of Cav-1 Overexpressing H460 cells and Melanoma G361 Cells

To substantiate the role of ROS in cell migration and invasion, experiments were performed in Cav-1 overexpressing $\mathrm{H} 460$ cells described earlier and in melanoma G361 cells to determine cell type-specific effects of ROS. The cells were treated with the ROS modulators DMNQ, $\mathrm{H}_{2} \mathrm{O}_{2}$, and $\mathrm{FeSO}_{4}$, and their effects on cell migration and invasion were examined. The results show that DMNQ and $\mathrm{H}_{2} \mathrm{O}_{2}$ had similar inhibitory effects on cell migration and invasion in these cells, while $\mathrm{FeSO}_{4}$ had opposite effects (Fig. 8). These results are consistent with the earlier findings in H460 cells (Fig. 6) and suggest the generality of the observed ROS effects on cell migration and invasion. 


\section{Discussion}

The role of ROS in the regulation of cell migration and invasion has been described (Laurent, Nicco et al. 2005; Storz 2005), but the role of specific ROS and their regulatory mechanisms have not been well investigated. We report here that different ROS have different effects on cell migration and invasion in human lung carcinoma $\mathrm{H} 460$ cells and melanoma G361 cells. $\mathrm{O}_{2}{ }^{-}$and $\mathrm{H}_{2} \mathrm{O}_{2}$ suppress the migration and invasion of the cells, whereas $\mathrm{OH} \cdot$ promotes the cell motility activities. Since several ROS are generated during oxidative stress which has been linked to tumor progression, the results of this study further indicate that depending on the type and abundance of specific ROS generated, oxidative stress conditions may promote or suppress tumor progression by affecting cell migration and invasion.

The present study also demonstrates the role of Cav-1 as a key target of ROS regulation of cell motility. Cav-1 has been shown to be involved in cancer cell motility and tumor progression (Fiucci, Ravid et al. 2002; Sloan, Stanley et al. 2004; Williams and Lisanti 2004; Joshi, Strugnell et al. 2008). However, its precise role and regulatory mechanisms are still unclear as both promoting and inhibitory roles of Cav-1 have been reported. In pancreatic cancer cells, Cav-1 was shown to inhibit cell migration and invasion through the inactivation of RhoC GTPase and Erk-MMP signaling pathways (Han and Zhu; Lin, DiVito et al. 2005). A similar inhibitory effect of Cav-1 was observed in breast cancer MTLn3 and MCF-7 cells (Zhang, Razani et al. 2000; Fiucci, Ravid et al. 2002). In contrast, Cav-1 has been reported to promote lung cancer cell invasion by mediating 
filopodia formation (Ho, Huang et al. 2002). Furthermore, Cav-1 expression is associated with the tumor grade and metastasis of non-small cell lung cancer (NSCLC) (Kato, Miyamoto et al. 2004; Moon, Lee et al. 2005). Consistent with the tumor promoting role of Cav-1, we found that Cav-1 promotes the migration and invasion of NSCLC H460 cells. The function of Cav-1 is closely associated with its expression level. Although the difference in Cav-1 expression may result from various factors such as cell type and stage of cancer (Shatz and Liscovitch 2008), the tumor microenvironment and oxidative status seem to play a key role.

Several ROS have been shown to be up-regulated in the tumor microenvironment and have been implicated in the aggressive behaviors of tumor cells (Laurent, Nicco et al. 2005; Storz 2005). However, the mechanisms by which ROS regulate Cav-1 and tumor cell migration and invasiveness have not been thoroughly explored. In the present study, we demonstrate that ROS play an important role in regulating Cav-1 expression and cell migratory functions in human lung cancer $\mathrm{H} 460$ cells. We also show the positive correlation between Cav-1 expression and cell motility in these cells. More importantly, we demonstrate the differential roles of individual ROS in Cav-1 expression and cell migration with $\mathrm{O}_{2}{ }^{-}$and $\mathrm{H}_{2} \mathrm{O}_{2}$ having a negative regulatory role, and $\mathrm{OH}$. playing a positive role.

The results of this study also indicate that the effect of ROS on cell migratory functions is dependent on Cav-1 expression and is associated with Akt activity. Activation of Akt by Cav-1 has been shown to mediate cancer cell migration (Ravid, Maor et al. 2005; Li, Ren et al. 2009; Park and Han 2009) and 
is likely to play an important role in the ROS-induced effects on cell motility alterations. ROS may also regulate cell motility through other Cav-1-dependent mechanisms. For example, recent studies have shown that oxidative stress induced Cav-1 phosphorylation at tyrosine 14 (Chen, Li et al. 2005; Sun, Hu et al. 2009), which has been proposed to play a role in cancer cell migration through the regulation of focal adhesion (Joshi, Strugnell et al. 2008).

The expression of Cav-1 is tightly regulated at various levels, including transcriptional and post-transcriptional [for review, see (Williams and Lisanti 2004)]. We found that the down-regulation of Cav-1 by $\mathrm{O}_{2}{ }^{-}$and $\mathrm{H}_{2} \mathrm{O}_{2}$ was not due to transcriptional inactivation since Cav-1 mRNA expression was not downregulated but slightly increased by the $\mathrm{O}_{2}{ }^{-}$inducer $\mathrm{DMNQ}$ and $\mathrm{H}_{2} \mathrm{O}_{2}$, possibly due to stress induction. Instead, such down-regulation was shown to be mediated by protein degradation via the ubiquitin-proteasome pathway. The negative regulatory roles of $\mathrm{O}_{2}{ }^{-}$and $\mathrm{H}_{2} \mathrm{O}_{2}$ in Cav-1 expression and cell motility were confirmed by the observations that the scavengers of $\mathrm{O}_{2}{ }^{-}$and $\mathrm{H}_{2} \mathrm{O}_{2}(\mathrm{MnTBAP}$ and catalase) were able to reverse the effects of DMNQ and $\mathrm{H}_{2} \mathrm{O}_{2}$. These results are consistent with human clinical data showing an increase in MnSOD and catalase activities in patients with NSCLC (Chung-man Ho, Zheng et al. 2001). Such increases may result in decreased $\mathrm{O}_{2}{ }^{-}$and $\mathrm{H}_{2} \mathrm{O}_{2}$ levels and elevated Cav-1 and migratory activities.

The promoting role of $\mathrm{OH}$. in cancer cell migration and Cav-1 expression is unexpected and suggests the potential important role of this oxidative species in tumor progression and metastasis. To our knowledge, this is the first 
demonstration of the positive regulatory role of $\mathrm{OH} \cdot$ in cancer cell motility and Cav-1 expression. This finding also suggests that elevated $\mathrm{OH}$. levels may increase the risk of cancer metastasis. $\mathrm{OH}$. is a highly reactive free radical generated primarily via a biologic Fenton reaction. In addition to its known ability to induce lipid peroxidation and DNA damage, recent studies suggest that it may exert its cellular effects through the alterations of several key proteins such as p53 and MMPs (Jiang, Wei et al. 2007). The mechanisms of $\mathrm{OH}$. regulation of cancer cell migration and invasiveness are not known and likely involve several signaling pathways. Our study provides initial evidence that this regulation is mediated, at least in part, through Cav-1 up-regulation.

In conclusion, we demonstrate that Cav-1 plays an important role as a positive regulator of cancer migration and invasion in human lung carcinoma H460 cells. Our results also reveal the differential roles of individual ROS in cancer cell motility and Cav-1 expression, which could be important in understanding tumor progression and metastasis. Our results also provide evidence supporting the role of $\mathrm{OH} \cdot$ as a key determinant and positive regulator of cancer cell invasiveness. This new finding suggests a linkage between elevated $\mathrm{OH}$. formation and increased risk of tumor formation and metastasis. 


\section{References}

Alexandrova, A. Y., P. B. Kopnin, et al. (2006). "ROS up-regulation mediates Ras-induced changes of cell morphology and motility." Exp Cell Res 312(11): 2066-73.

Bacac, M. and I. Stamenkovic (2008). "Metastatic cancer cell." Annu Rev Pathol 3: 221-47.

Cassoni, P., L. Daniele, et al. (2009). "Caveolin-1 expression in lung carcinoma varies according to tumour histotype and is acquired de novo in brain metastases." Histopathology 55(1): 20-7.

Chanvorachote, P., U. Nimmannit, et al. (2009). "Nitric oxide regulates lung carcinoma cell anoikis through inhibition of ubiquitin-proteasomal degradation of caveolin-1." J Biol Chem 284(41): 28476-84.

Chen, D. B., S. M. Li, et al. (2005). "Tyrosine phosphorylation of caveolin 1 by oxidative stress is reversible and dependent on the c-src tyrosine kinase but not mitogen-activated protein kinase pathways in placental artery endothelial cells." Biol Reprod 73(4): 761-72.

Chiarugi, P., G. Pani, et al. (2003). "Reactive oxygen species as essential mediators of cell adhesion: the oxidative inhibition of a FAK tyrosine

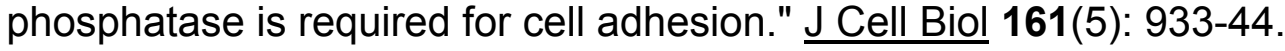

Chung-man Ho, J., S. Zheng, et al. (2001). "Differential expression of manganese superoxide dismutase and catalase in lung cancer." $\underline{\text { Cancer }}$ Res 61(23): 8578-85.

Dokka, S., X. Shi, et al. (2001). "Interleukin-10-mediated inhibition of free radical generation in macrophages." Am J Physiol Lung Cell Mol Physiol 280(6): L1196-202.

Droge, W. (2002). "Free radicals in the physiological control of cell function." Physiol Rev 82(1): 47-95.

Esme, H., M. Cemek, et al. (2008). "High levels of oxidative stress in patients with advanced lung cancer." Respirology 13(1): 112-6. 
Fiucci, G., D. Ravid, et al. (2002). "Caveolin-1 inhibits anchorage-independent growth, anoikis and invasiveness in MCF-7 human breast cancer cells." Oncogene 21(15): 2365-75.

Friedl, P. and K. Wolf (2003). "Tumour-cell invasion and migration: diversity and escape mechanisms." Nat Rev Cancer 3(5): 362-74.

Grande-Garcia, A., A. Echarri, et al. (2007). "Caveolin-1 regulates cell polarization and directional migration through Src kinase and Rho GTPases." J Cell Biol 177(4): 683-94.

Gupta, G. P. and J. Massague (2006). "Cancer metastasis: building a framework." Cell 127(4): 679-95.

Han, F. and H. G. Zhu "Caveolin-1 regulating the invasion and expression of matrix metalloproteinase (MMPs) in pancreatic carcinoma cells." $\underline{\mathrm{J} \text { Surg }}$ Res 159(1): 443-50.

Herr, B., J. Zhou, et al. (2007). "The interaction of superoxide with nitric oxide destabilizes hypoxia-inducible factor-1alpha." Cell Mol Life Sci 64(24): 3295-305.

Hileman, E. O., J. Liu, et al. (2004). "Intrinsic oxidative stress in cancer cells: a biochemical basis for therapeutic selectivity." Cancer Chemother Pharmacol 53(3): 209-19.

Ho, C. C., P. H. Huang, et al. (2002). "Up-regulated caveolin-1 accentuates the metastasis capability of lung adenocarcinoma by inducing filopodia formation." Am J Pathol 161(5): 1647-56.

Ishihara, Y., D. Shiba, et al. (2006). "Enhancement of DMNQ-induced hepatocyte toxicity by cytochrome P450 inhibition." Toxicol Appl Pharmacol 214(2): $109-17$.

Jiang, M., Q. Wei, et al. (2007). "Effects of hydroxyl radical scavenging on cisplatin-induced p53 activation, tubular cell apoptosis and nephrotoxicity." Biochem Pharmacol 73(9): 1499-510. 
Joshi, B., S. S. Strugnell, et al. (2008). "Phosphorylated caveolin-1 regulates Rho/ROCK-dependent focal adhesion dynamics and tumor cell migration and invasion." Cancer Res 68(20): 8210-20.

Kato, T., M. Miyamoto, et al. (2004). "Difference of caveolin-1 expression pattern in human lung neoplastic tissue. Atypical adenomatous hyperplasia, adenocarcinoma and squamous cell carcinoma." Cancer Lett 214(1): 1218.

Kopfstein, L. and G. Christofori (2006). "Metastasis: cell-autonomous mechanisms versus contributions by the tumor microenvironment." Cell Mol Life Sci 63(4): 449-68.

Kumar, B., S. Koul, et al. (2008). "Oxidative stress is inherent in prostate cancer cells and is required for aggressive phenotype." Cancer Res 68(6): 177785.

Laurent, A., C. Nicco, et al. (2005). "Controlling tumor growth by modulating endogenous production of reactive oxygen species." Cancer Res 65(3): 948-56.

Lee, K. J., S. J. Hwang, et al. (2008). "Saponins derived from the roots of Platycodon grandiflorum inhibit HT-1080 cell invasion and MMPs activities: regulation of NF-kappaB activation via ROS signal pathway." Cancer Lett 268(2): 233-43.

Li, L., C. Ren, et al. (2009). "Caveolin-1 promotes autoregulatory, Akt-mediated induction of cancer-promoting growth factors in prostate cancer cells." Mol Cancer Res 7(11): 1781-91.

Lin, M., M. M. DiVito, et al. (2005). "Regulation of pancreatic cancer cell migration and invasion by RhoC GTPase and caveolin-1." Mol Cancer 4(1): 21.

Misthos, P., S. Katsaragakis, et al. (2005). "Postresectional pulmonary oxidative stress in lung cancer patients. The role of one-lung ventilation." Eur J Cardiothorac Surg 27(3): 379-82; discussion 382-3. 
Moon, K. C., G. K. Lee, et al. (2005). "Expression of caveolin-1 in pleomorphic carcinoma of the lung is correlated with a poor prognosis." Anticancer Res 25(6C): 4631-7.

Nelson, K. K. and J. A. Melendez (2004). "Mitochondrial redox control of matrix metalloproteinases." Free Radic Biol Med 37(6): 768-84.

Novo, E., F. Marra, et al. (2006). "Dose dependent and divergent effects of superoxide anion on cell death, proliferation, and migration of activated human hepatic stellate cells." Gut 55(1): 90-7.

Novo, E., F. Marra, et al. (2006). "Overexpression of Bcl-2 by activated human hepatic stellate cells: resistance to apoptosis as a mechanism of progressive hepatic fibrogenesis in humans." Gut 55(8): 1174-82.

O'Toole, E. A., M. Goel, et al. (1996). "Hydrogen peroxide inhibits human keratinocyte migration." Dermatol Surg 22(6): 525-9.

Oka, N., M. Yamamoto, et al. (1997). "Caveolin interaction with protein kinase C. Isoenzyme-dependent regulation of kinase activity by the caveolin scaffolding domain peptide." J Biol Chem 272(52): 33416-21.

Oka, T., R. Yamamoto, et al. (1997). "Three vha genes encode proteolipids of Caenorhabditis elegans vacuolar-type ATPase. Gene structures and preferential expression in an $\mathrm{H}$-shaped excretory cell and rectal cells." $\underline{\mathrm{J}}$ Biol Chem 272(39): 24387-92.

Park, J. H. and H. J. Han (2009). "Caveolin-1 plays important role in EGFinduced migration and proliferation of mouse embryonic stem cells: involvement of PI3K/Akt and ERK." Am J Physiol Cell Physiol 297(4): C935-44.

Payne, J. E., C. Bonnefous, et al. "Discovery of dual inducible/neuronal nitric oxide synthase (iNOS/nNOS) inhibitor development candidate 4-((2cyclobutyl-1H-imidazo[4,5-b]pyrazin-1-yl)methyl)-7,8-difluoroquinoli n$2(1 \mathrm{H})$-one (KD7332) part 2: identification of a novel, potent, and selective series of benzimidazole-quinolinone iNOS/nNOS dimerization inhibitors

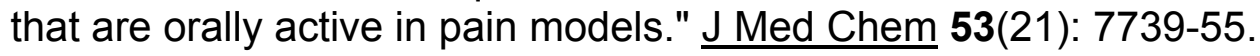


Ravid, D., S. Maor, et al. (2005). "Caveolin-1 inhibits cell detachment-induced p53 activation and anoikis by upregulation of insulin-like growth factor-I receptors and signaling." Oncogene 24(8): 1338-47.

Shatz, M. and M. Liscovitch (2008). "Caveolin-1: a tumor-promoting role in human cancer." Int J Radiat Biol 84(3): 177-89.

Sloan, E. K., K. L. Stanley, et al. (2004). "Caveolin-1 inhibits breast cancer growth and metastasis." Oncogene 23(47): 7893-7.

Steiling, H., B. Munz, et al. (1999). "Different types of ROS-scavenging enzymes are expressed during cutaneous wound repair." Exp Cell Res 247(2): 48494.

Storz, P. (2005). "Reactive oxygen species in tumor progression." Front Biosci 10: 1881-96.

Sun, Y., G. Hu, et al. (2009). "Phosphorylation of caveolin-1 regulates oxidantinduced pulmonary vascular permeability via paracellular and transcellular pathways." Circ Res 105(7): 676-85, 15 p following 685.

Svineng, G., C. Ravuri, et al. (2008). "The role of reactive oxygen species in integrin and matrix metalloproteinase expression and function." Tissue Res 49(3): 197-202.

Szatrowski, T. P. and C. F. Nathan (1991). "Production of large amounts of hydrogen peroxide by human tumor cells." Cancer Res 51(3): 794-8.

Tobar, N., M. Caceres, et al. (2008). "RAC1 activity and intracellular ROS modulate the migratory potential of MCF-7 cells through a NADPH oxidase and NFkappaB-dependent mechanism." Cancer Lett 267(1): 12532.

Urbich, C., E. Dernbach, et al. (2002). "CD40 ligand inhibits endothelial cell migration by increasing production of endothelial reactive oxygen species." Circulation 106(8): 981-6. 
Ushio-Fukai, M. and R. W. Alexander (2004). "Reactive oxygen species as mediators of angiogenesis signaling: role of $\mathrm{NAD}(\mathrm{P}) \mathrm{H}$ oxidase." Mol Cell Biochem 264(1-2): 85-97.

Volonte, D., K. Zhang, et al. (2002). "Expression of caveolin-1 induces premature cellular senescence in primary cultures of murine fibroblasts." Mol Biol Cell 13(7): 2502-17.

Wang, S., S. S. Leonard, et al. (2004). "Role of reactive oxygen species and $\mathrm{Cr}(\mathrm{VI})$ in Ras-mediated signal transduction." Mol Cell Biochem 255(1-2): $119-27$.

Williams, T. M., G. S. Hassan, et al. (2005). "Caveolin-1 promotes tumor progression in an autochthonous mouse model of prostate cancer: genetic ablation of Cav-1 delays advanced prostate tumor development in tramp mice." J Biol Chem 280(26): 25134-45.

Williams, T. M. and M. P. Lisanti (2004). "The Caveolin genes: from cell biology to medicine." Ann Med 36(8): 584-95.

Yoo, S. H., Y. S. Park, et al. (2003). "Expression of caveolin-1 is associated with poor prognosis of patients with squamous cell carcinoma of the lung." Lung Cancer 42(2): 195-202.

Zhang, W., B. Razani, et al. (2000). "Caveolin-1 inhibits epidermal growth factorstimulated lamellipod extension and cell migration in metastatic mammary adenocarcinoma cells (MTLn3). Transformation suppressor effects of adenovirus-mediated gene delivery of caveolin-1." J Biol Chem 275(27): 20717-25. 


\section{FIGURES}

Figure 1
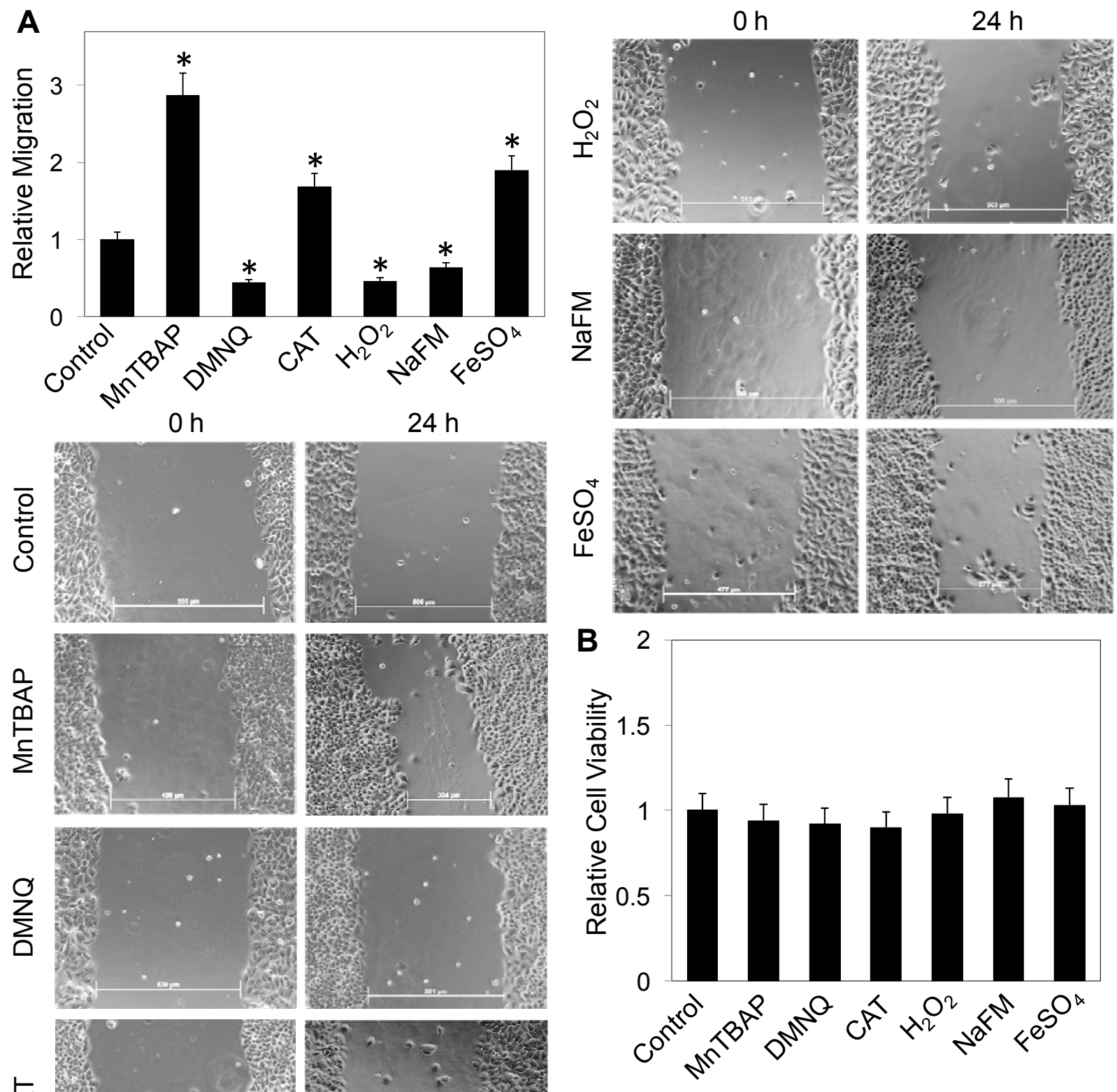
FIGURE 1. ROS regulate migration of human lung epithelial $\mathbf{H} 460$ cells. $A$, confluent monolayers of $\mathrm{H} 460$ cells were wounded and the cells were allowed to migrate for 24 hours in the presence or absence of various ROS modulators, including MnTBAP $(50 \mu \mathrm{M})$, DMNQ $(5 \mu \mathrm{M})$, CAT (7,500 U/ml), $\mathrm{H}_{2} \mathrm{O}_{2}(100 \mu \mathrm{M})$, $\mathrm{NaFM}(5 \mathrm{mM})$, and $\mathrm{FeSO}_{4}(50 \mu \mathrm{M})$. Wound space was visualized under a phasecontrast microscope and analyzed by comparing the relative change in wound space of the treated over non-treated cell monolayers. Representative micrographs from four independent experiments are shown. $B$, effect of ROS modulators on cell viability. Cell monolayers were similarly treated with the indicated concentrations of ROS modulators and analyzed for cell viability after 24 hours by MTT assay. Data are the mean \pm S.D. $(n=4) .{ }^{*}, p<0.05$ versus non-treated control. 
Figure 2
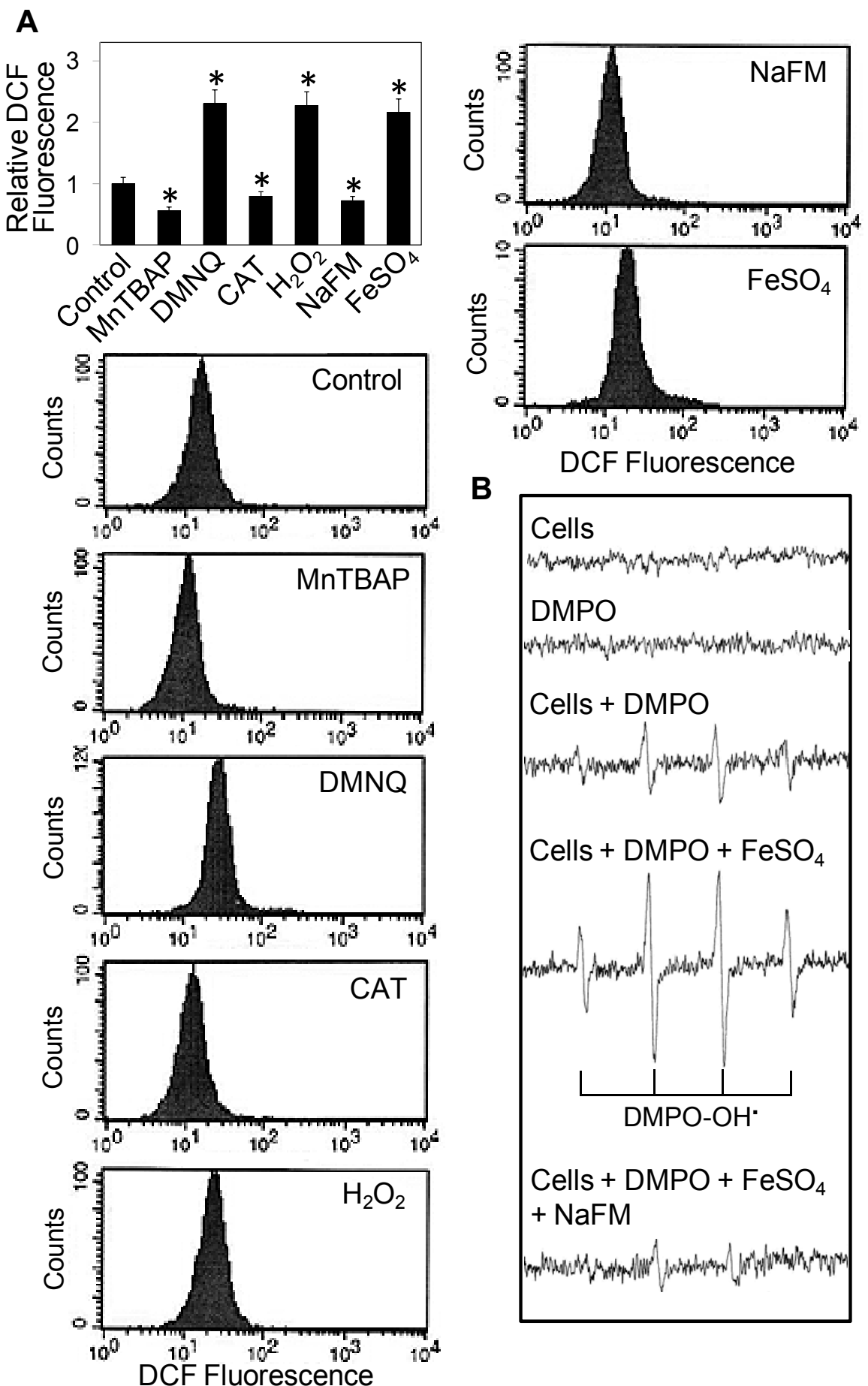

Cells + DMPO + $\mathrm{FeSO}_{4}$

$+\mathrm{NaFM}$

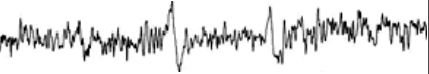


FIGURE 2. Effect of ROS modulators on cellular ROS. A, H460 cells were treated with the indicated concentrations of ROS modulators as shown in Figure 1 and analyzed for ROS levels by flow cytometry using $\mathrm{H}_{2}$ DCF-DA as a fluorescent probe. Data shown are relative fluorescence intensities over control level determined at 2 hours post-treatment. B, ESR detection of ROS. H460 cells $\left(1 \times 10^{6} \mathrm{cells} / \mathrm{ml}\right)$ were incubated in culture medium containing the spin trapper DMPO $(100 \mathrm{mM})$ with or without $\mathrm{FeSO}_{4}(50 \mu \mathrm{M})$ and $\mathrm{NaFM}(5 \mathrm{mM})$. ESR spectra were recorded 10 minutes after the addition of the test agents. The spectrometer settings were as follows: receiver gain at $2.5 \times 10^{4}$, time constants at 0.04 seconds, modulation amplitude at $1.0 \mathrm{G}$, scan time at 42 seconds, magnetic field at $3475 \pm 100 \mathrm{G}$. Data are the mean \pm S.D. $(n=3) .{ }^{*}, p<0.05$ versus nontreated control. 
Figure 3

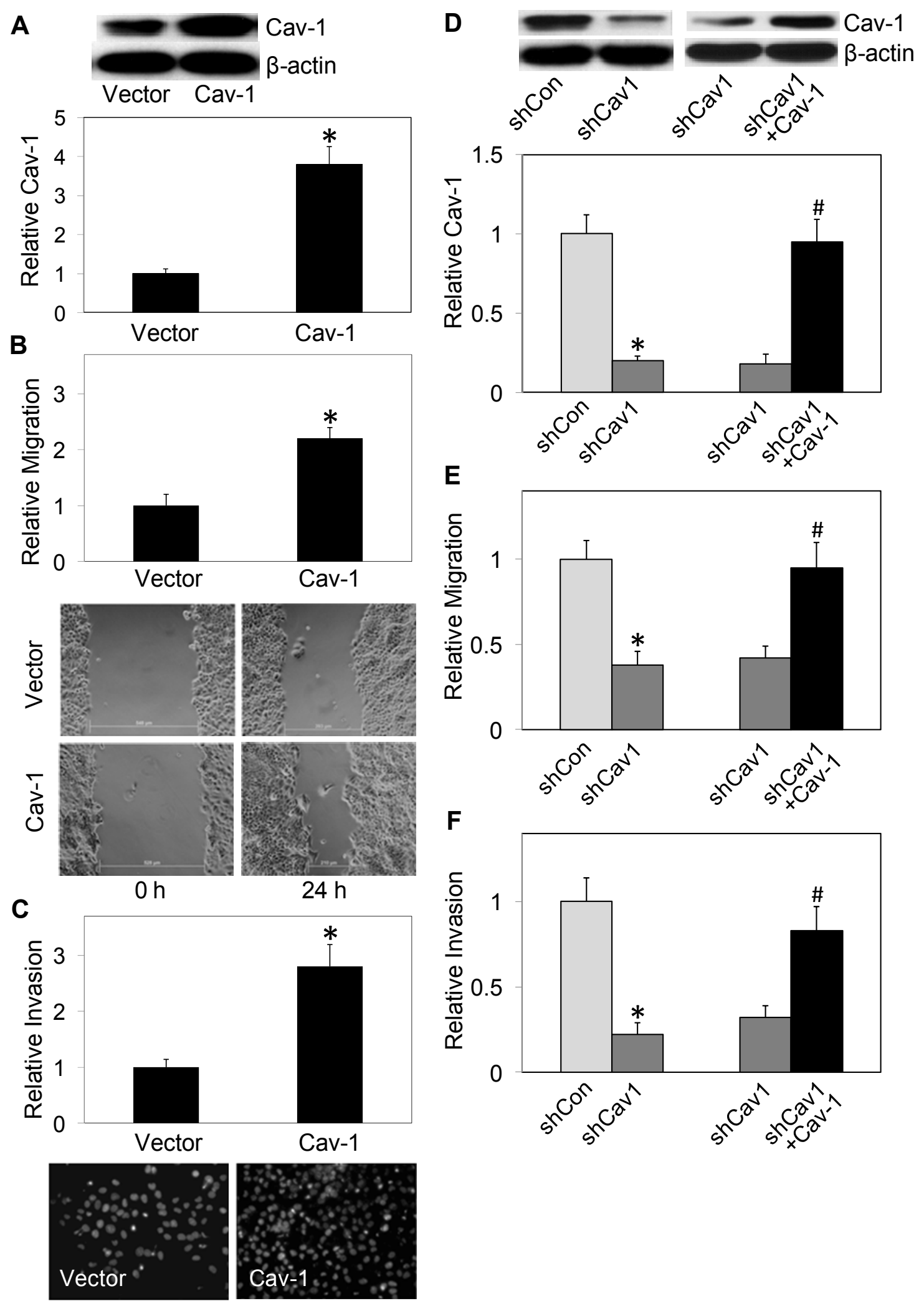




\section{FIGURE 3. Effects of Cav-1 overexpression and knockdown on cell}

migration and invasion. $\mathrm{H} 460$ cells were stably transfected with Cav-1 or control plasmid as described under "Materials and Methods". A, Cav-1 expression in the control and Cav-1-transfected cells was determined by Western blotting. Cell extracts were prepared and separated on $10 \%$ polyacrylamide-SDS gels, transferred, and probed with Cav-1 antibody. $\beta$-actin was used as a loading control. $B$, effect of Cav-1 overexpression on cell migration. Cav-1 and controltransfected cells were cultured in 24-well plates and analyzed for cell migration at 24 hours by wound assay. $C$, effect of Cav-1 overexpression on cell invasion. Cav-1 and control-transfected cells were added to extracellular matrix-coated inserts in a Transwell chamber and incubated for 24 hours. Invading cells were counted under a fluorescence microscope after staining with Hoechst 33342 and the average number of cells was scored in each case. $D-F$, Cav-1 knockdown experiments were performed using $\mathrm{H} 460$ cells treated with Cav-1 shRNA (shCav1) viral particles or control shRNA (shCon) particles as described under "Materials and Methods". D, Cav-1 expression in shCav-1 and shCon-treated cells determined by Western blotting at 36 hours post-treatment (left panel). Rescue experiment was performed in shCav-1-treated cells by transfecting the cells with Cav-1 plasmid as described above and analyzed for Cav-1 expression by Western blotting (right panel). $E$ and $F$, migration and invasion of shCon, shCav-1, and rescued cells determined by wound and Transwell assays, respectively. Data are the mean \pm S.D. $(n=3) .{ }^{*}, p<0.05$ versus control transfection; ${ }^{\#}, p<0.05$ versus shCav1control. 
Figure 4

A

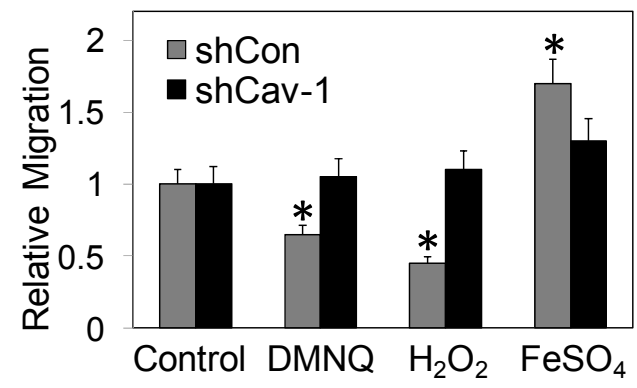

C
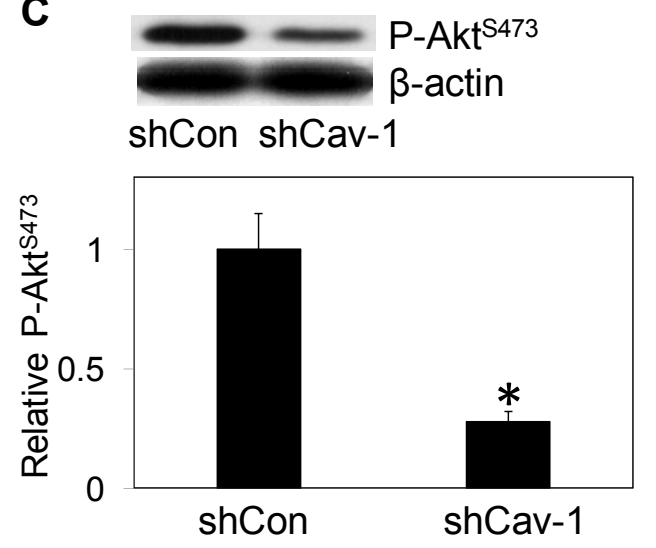

B
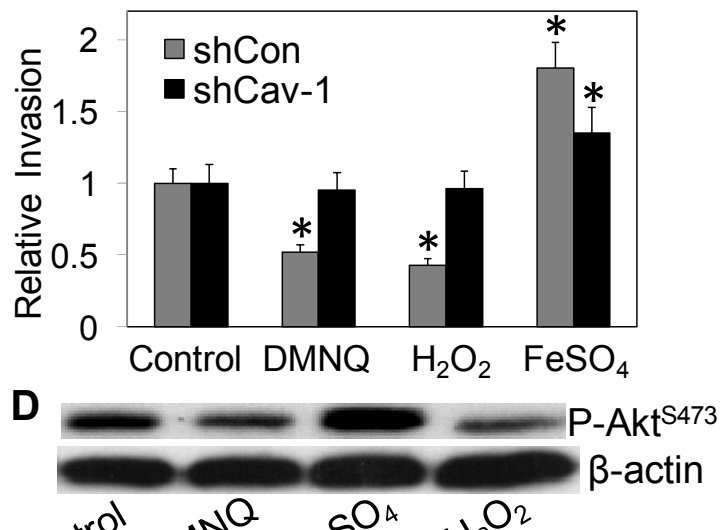

FIGURE 4. Effects of Cav-1 knockdown on ROS-modulated cell migration, invasion, and Akt activity. $A$ and $B$, shCav-1 and shCon cells were treated with DMNQ $(5 \mu \mathrm{M}), \mathrm{H}_{2} \mathrm{O}_{2}(100 \mu \mathrm{M})$, and $\mathrm{FeSO}_{4}(50 \mu \mathrm{M})$ for 24 hours and analyzed for cell migration and invasion. $C$, shCav-1 and shCon cells were analyzed for Akt phosphorylation by Western blotting. Blots were probed with phospho-Akt Ser473 antibody and reprobed with $\beta$-actin antibody. D, H460 cells were treated with 
DMNQ $(5 \mu \mathrm{M}), \mathrm{H}_{2} \mathrm{O}_{2}(100 \mu \mathrm{M})$ and $\mathrm{FeSO}_{4}(50 \mu \mathrm{M})$, and analyzed for Akt phosphorylation at 6 hours post-treatment. Data are the mean \pm S.D. $(n=3){ }^{*}, p$ $<0.05$ versus control cells. 
Figure 5

A

Cav-1

$\beta$-actin
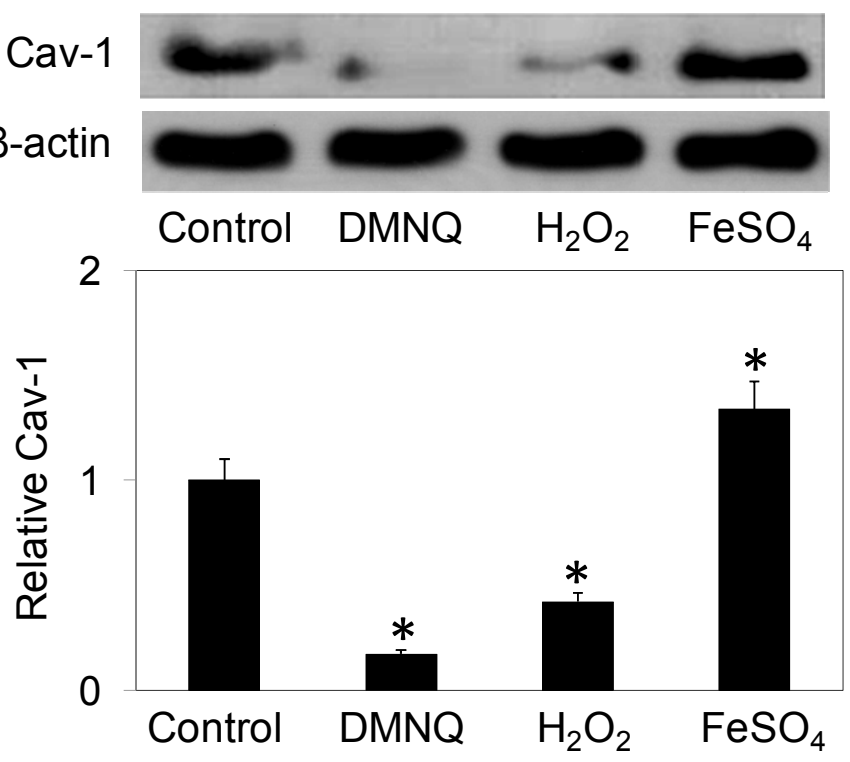

B

Cav-1

$\beta$-actin
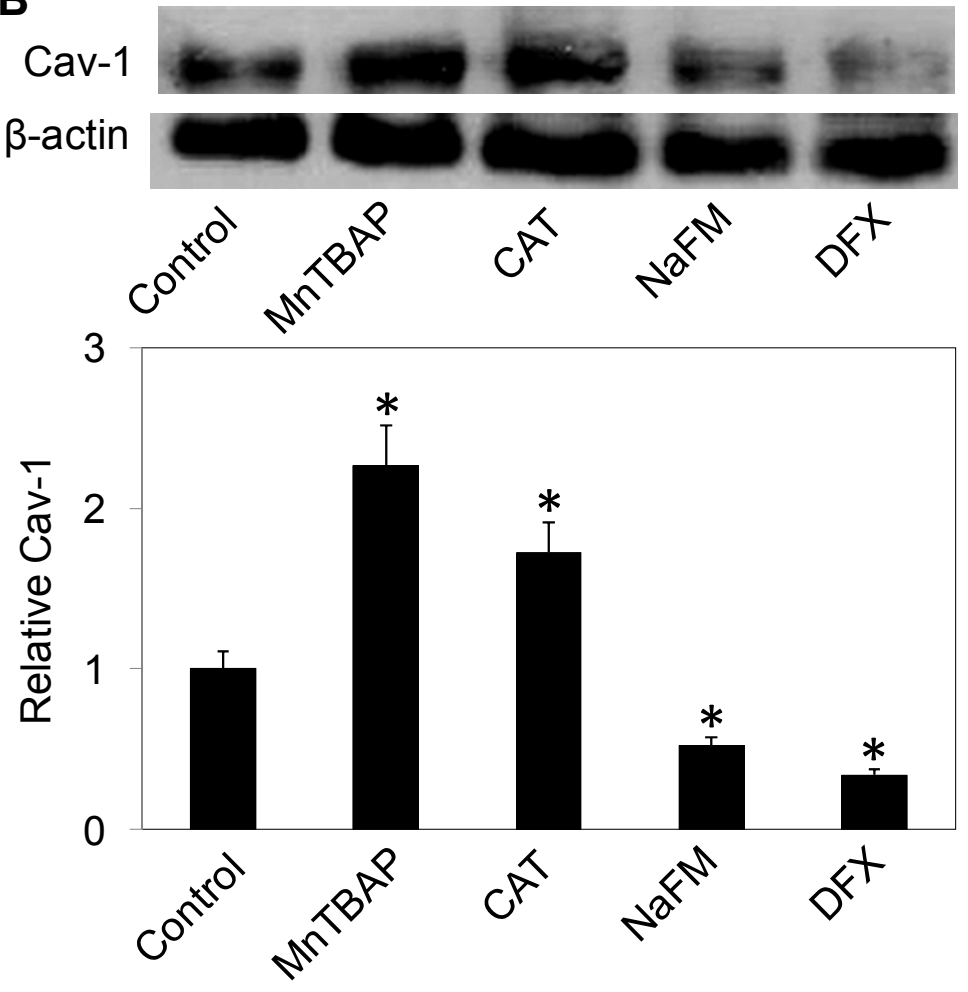

FIGURE 5. ROS regulate Cav-1 expression. $A, \mathrm{H} 460$ cells were treated with various ROS generators, including DMNQ $(5 \mu \mathrm{M}), \mathrm{H}_{2} \mathrm{O}_{2}(100 \mu \mathrm{M})$, and $\mathrm{FeSO}_{4}$ 
$(50 \mu \mathrm{M})$ for 24 hours, and cell lysates were prepared and analyzed for Cav-1 expression by Western blotting. $B$, cells were treated with various ROS scavengers or inhibitors, including MnTBAP (50 $\mu \mathrm{M})$, CAT (7,500 U/ml), NaFM (5 $\mathrm{mM})$, and DFX (0.5 mM), and Cav-1 expression was determined after 24 hours. Blots were reprobed with $\beta$-actin antibody to confirm equal loading of samples. The immunoblot signals were quantified by densiometry, and mean data from three independent experiments (one of which is shown here) was normalized to the result obtained in control cells. Data are the mean \pm S.D. $(n=3) .{ }^{*}, p<0.05$ versus non-treated control. 
Figure 6

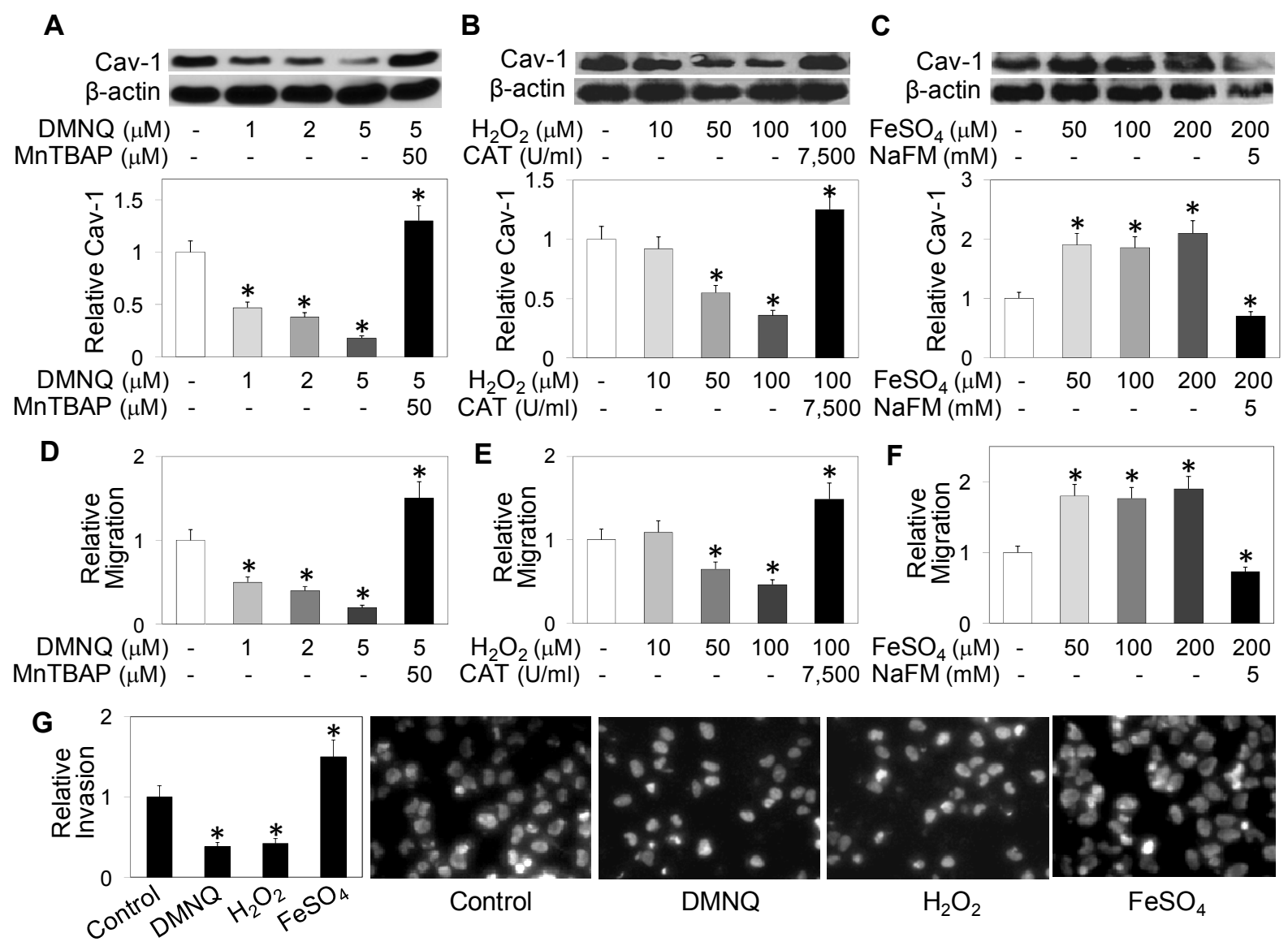

FIGURE 6. Differential effects of ROS on Cav-1 expression, cell migration and invasion. Western blot analysis of Cav-1 expression in $\mathrm{H} 460$ cells treated with the indicated concentrations of $A$, DMNQ and MnTBAP; $B, \mathrm{H}_{2} \mathrm{O}_{2}$ and CAT; and $\mathrm{C}, \mathrm{FeSO}_{4}$ and $\mathrm{NaFM}$ for 24 hours. Cell migration was determined 24 hours after the treatment with $D, \mathrm{DMNQ}$ and MnTBAP; $E, \mathrm{H}_{2} \mathrm{O}_{2}$ and CAT; and $F, \mathrm{FeSO}_{4}$ and NaFM. G, cell invasion determined 24 hours after the treatment with DMNQ $(5 \mu \mathrm{M}), \mathrm{H}_{2} \mathrm{O}_{2}(100 \mu \mathrm{M})$, and $\mathrm{FeSO}_{4}(50 \mu \mathrm{M})$. Data are the mean \pm S.D. $(n=3) .{ }^{*}$, $p<0.05$ versus non-treated control. 
Figure 7
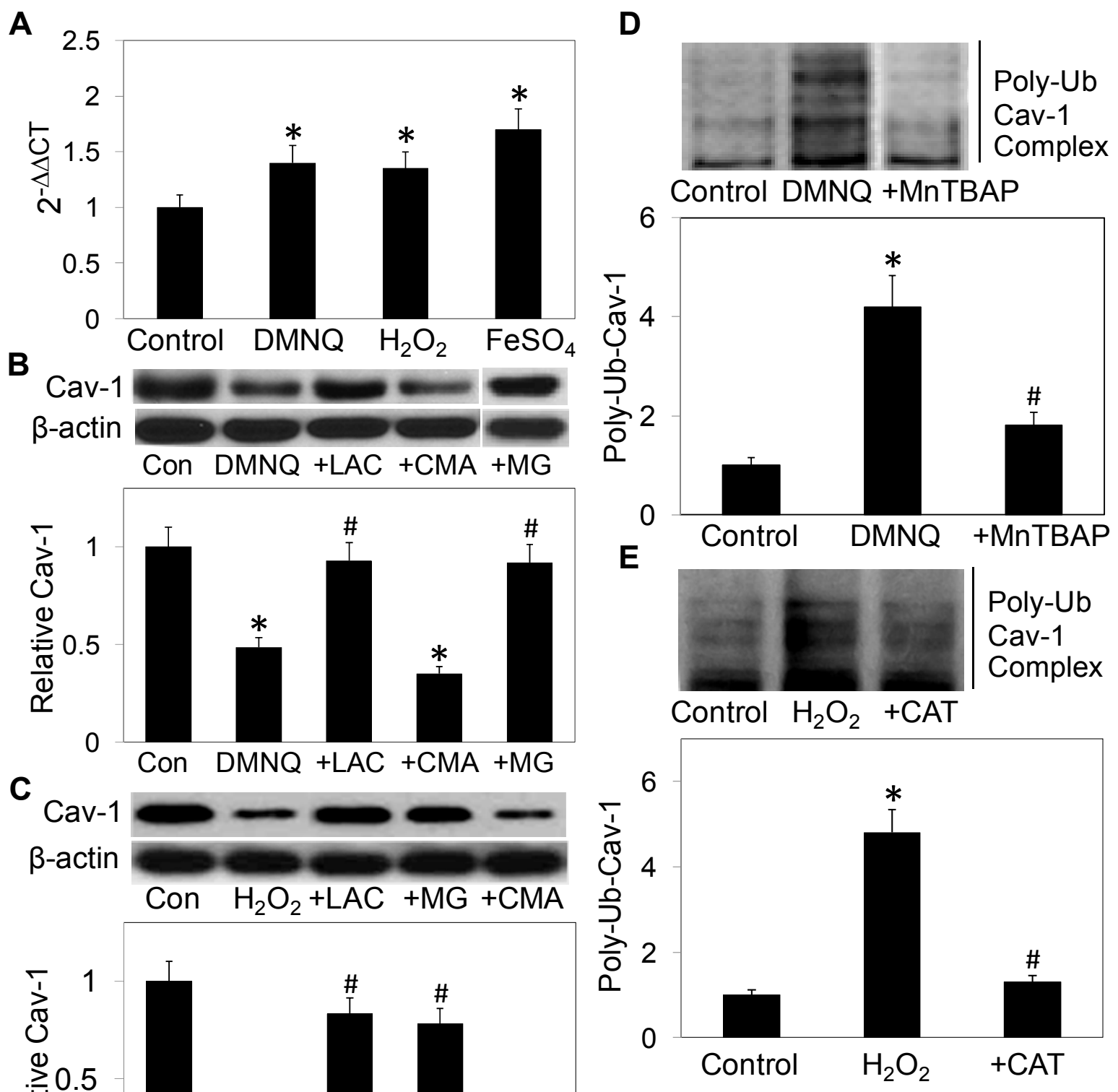


\section{FIGURE 7. Effects of ROS on Cav-1 mRNA expression and protein}

degradation. $A, \mathrm{H} 460$ cells were either left untreated or treated with DMNQ (5 $\mu \mathrm{M}), \mathrm{H}_{2} \mathrm{O}_{2}(100 \mu \mathrm{M})$, or $\mathrm{FeSO}_{4}(50 \mu \mathrm{M})$ for 24 hours. Cav-1 and GAPDH mRNA expression were then determined by quantitative real-time PCR. The relative mRNA expression was determined by using the comparative CT method as described under "Materials and Methods." $B$ and $C, \mathrm{H} 460$ cells were pretreated with proteasome inhibitor lactacystin $(L A C)(10 \mu \mathrm{M}), \mathrm{MG} 132(25 \mu \mathrm{M})$, or with lysosome inhibitor concanamycin $A(C M A)(1 \mu \mathrm{M})$ for 1 hour and then treated with DMNQ $(5 \mu \mathrm{M})$ or $\mathrm{H}_{2} \mathrm{O}_{2}(100 \mu \mathrm{M})$ for 24 hour. Cav-1 expression was determined by Western blots using anti-Cav-1 antibody. $D$ and $E$, cells were treated with DMNQ $(5 \mu \mathrm{M})$ in the presence or absence of MnTBAP $(50 \mu \mathrm{M})$, or with $\mathrm{H}_{2} \mathrm{O}_{2}(100 \mu \mathrm{M})$ in the presence or absence of CAT $(7,500 \mathrm{U} / \mathrm{ml})$. Cell lysates were immunoprecipitated with anti-Cav-1 antibody and the immune complexes were analyzed for ubiquitin by Western blots using anti-ubiquitin antibody. Analysis of ubiquitin was performed at 2 hours post-treatment where ubiquitination was found to be maximal. Immunoblot signals were quantified by densiometry, and mean data from three independent experiments (one of which is shown here) was normalized to the result obtained in control cells. Data are the mean \pm S.D. $(n=3) .{ }^{*}, p<0.05$ versus non-treated control; ${ }^{\#}, p<0.05$ versus treated control. 
Figure 8

A

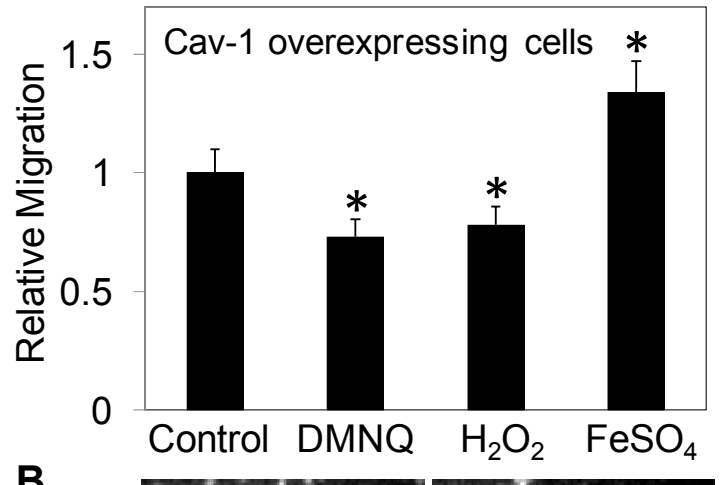

B
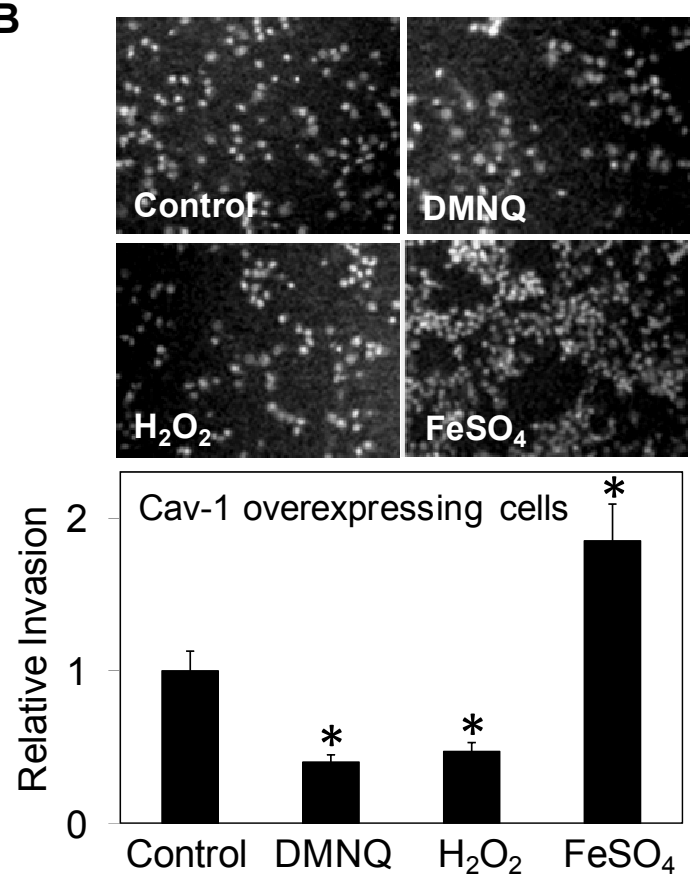

C

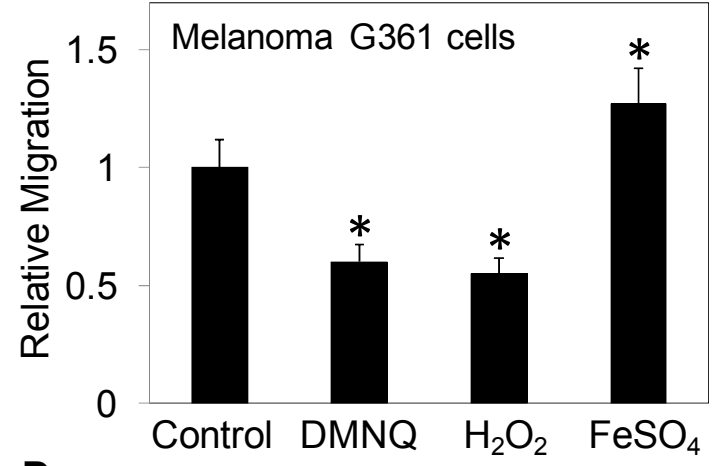

D
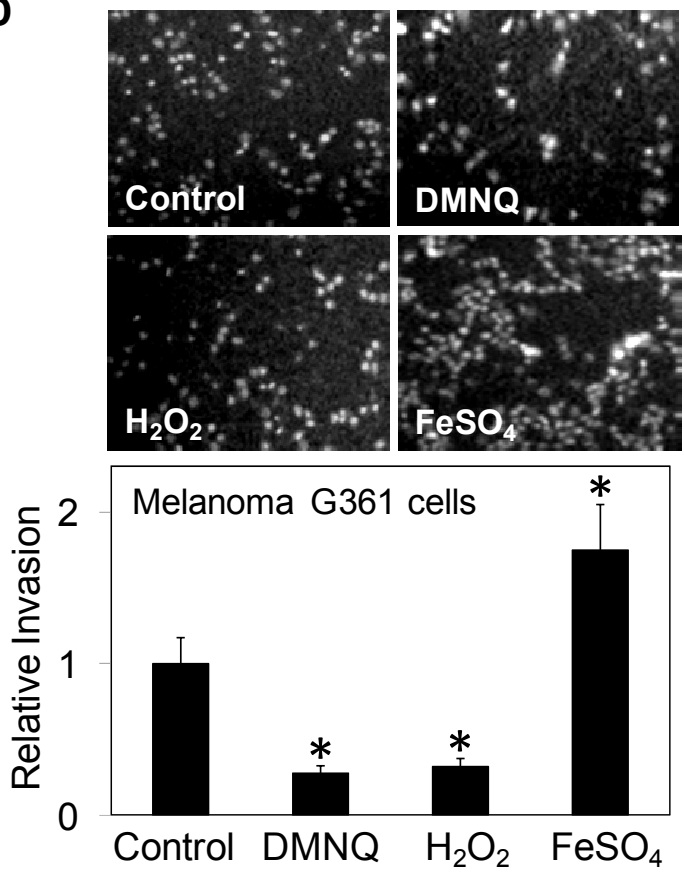

FIGURE 8. Effects of ROS modulators on cell migration and invasion in

Cav-1 overexpressing H460 cells and G361 melanoma cells. $A$ and $B$, migration and invasion of Cav-1 stably transfected H460 cells determined at 24 
hours after the treatment with DMNQ $(5 \mu \mathrm{M}), \mathrm{H}_{2} \mathrm{O}_{2}(100 \mu \mathrm{M})$, and $\mathrm{FeSO}_{4}(50$ $\mu \mathrm{M})$. Experiments were performed using wound assay and Transwell assay, respectively. $C$ and $D$, experiments were repeated using melanoma G361cells. Data are the mean \pm S.D. $(n=3) .{ }^{*}, p<0.05$ versus non-treated control. 


\section{Chapter 4. General Discussion and Conclusions}

\section{Overview}

The overall objective of this dissertation was to examine the effects of NO and ROS on different cellular signaling pathways that enhance tumorigenesis. Oxidative stress responses are part of normal physiological cellular processes of proliferation, senescence and cell death (Schrader and Fahimi 2006). However, chronic oxidative stress which occurs during prolonged inflammatory signaling can advance tumorigenesis (Bubici, Papa et al. 2006). NO and ROS are normally produced at low levels within the cell, however high levels are detected in cancer cells and within the tumor microenvironment (Pelicano, Carney et al. 2004). Hypoxia, increased iNOS activity, mitochondrial dysfunction and chronic cytokine and inflammatory signaling all lead to these increases. Additionally, many cancer therapeutics induce ROS production. The roles of anti- versus prooxidant therapies are widely debated throughout the clinical cancer field (Wang and Yi 2008), however the sub-lethal signaling effects or both NO and ROS are exceedingly important as they impact known tumorigenic pathways.

We were most interested in the effects of $\mathrm{NO}$ and ROS on existing tumor cells where aberrations in their signaling profiles are already noted. In chapter 2 , we examined NO changes on the antiapoptotic protein FLIP which is highly upregulated in chemotherapy-resistant cancers. In chapter 3 , we examined the 
effects of different ROS species on Cav-1-dependent migration in NSCLC cells.

Our results indicate that redox species play a vital role in known signaling pathways important in cancer cell behavior.

\section{S-nitrosylation of FLIP moderates its function}

S-nitrosylation is a PTM that modifies the activity of more than 100 target proteins (Hess, Matsumoto et al. 2005). Chapter 2 contains the details of our first study examining the effects of S-nitrosylation of FLIP residues 254 and 259. This project is especially novel since it demonstrates crosstalk between cell death and proliferative pathways involving FLIP, which is frequently upregulated in cancer. Many groups have shown that FLIP is a potent inhibitor of caspase-8mediated apoptosis when abundantly expressed, as seen in cancer (Igney and Krammer 2002). Our laboratory previously reported that S-nitrosylation of FLIP prevents ubiquitination and subsequent degradation promoting a more potent inhibition of Fas-mediated apoptosis (Chanvorachote, Nimmannit et al. 2005). In this study, we found that FLIP-mediated apoptotic resistance to DR-ligandinduced apoptosis is also dependent on the S-nitrosylation of FLIP at residues 254 and 259 (chapter 2, figure 3).

Additionally, FLIP has been reported to interact with proliferative, migratory and inflammatory pathways such as JNK, MAPK and NF-kB. We found that S-nitrosylation of FLIP mediates NF-KB activation, however we also 
demonstrate FLIPS (which lacks the S-nitrosylation residues) is capable of inducing NF-kB activity (chapter 2, figure 2). This is consistent with other groups (Golks, Brenner et al. 2006). Taken together, these results indicate FLIP mediates NF-kB activation via multiple signaling pathways. FLIP S-nitrosylation moderated the ability to bind NF-kB upstream activator RIP1 but not TRAF2 (chapter 2, figures 4, 5).

FLIP has been reported to be a substrate of caspase-8 following Fas stimulation (Niikura, Nonaka et al. 2002). Additionally, many proteins are known to be regulated by truncation. We found that S-nitrosylation of FLIP at residues 254 and 259 mediates processing into shorter forms (chapter 2, figure 6) further indicating potential functional effects of this PTM. Understanding the complete signaling profiles of potent antiapoptotic proteins that are frequently upregulated in cancer will help us to determine which proteins are most in need of targeting for effective drug therapies.

\section{Varying effects of ROS species on Cav-1-dependent migration in NSCLC cells}

A modest amount of ROS can promote cell proliferation and differentiation (Boonstra and Post 2004), whereas excessive amounts of ROS cause oxidative damage to lipids, proteins and DNA (Perry, Raina et al. 2000). These differences are the result of ROS acting as a secondary signaling molecule at low levels. 
Using specific ROS inhibitors and donors we were able to selectively modulate ROS species in cells (chapter 3 , figure 2) while remaining under toxicity thresholds (chapter 3, figure 1). Other studies have selectively shown $\mathrm{H}_{2} \mathrm{O}_{2}$ modulates tumor cell behavior through migration (Reineke, Liu et al.), NF-kB signaling (Shin, Zhao et al.), premature senescence (Yoshizaki, Fujiki et al. 2009) and apoptosis (Han, Hanawa et al. 2006) to name a few. Additionally, $\mathrm{O}_{2}^{-}$has been implicated in cancer, however most studies consider activity and expression levels of MnSOD. We found that $\mathrm{H}_{2} \mathrm{O}_{2}$ and $\mathrm{O}_{2}{ }^{-}$inhibited Cav-1dependent migration and invasion in NSCLC cells via destabilization of Cav-1 through the ubiquitin-proteosome pathway, however the exact mechanism is still unclear (chapter 3 , figures $1,5,7$ ). Conversely we found that highly reactive $\mathrm{OH}$. promoted migration and invasion through the potential stabilization or upregulation of Cav-1. The most common connections of $\mathrm{OH}$. with tumorigenesis are attributed to its potent oxidizing capacity. We demonstrate for the first time the association between $\mathrm{OH}$. and $\mathrm{Cav}$-1-dependent migration in cancer cells further indicating that specific imbalanes in redox biology within the cell can have dramatically divergent effects on cellular function.

\section{Future directions and recommendations}

Future studies examining the effects of ROS and NO on pathways in tumorigenesis are desperately needed. Countless pathways have been 
implicated in the pathogenesis of cancer and are nearly all subject to oxidative modification and both ROS and NO targeting. Unfortunately, redox biology is exceptionally difficult to manipulate and study in vivo and therefore cell culture experimentation with ROS and NO modification has been widely practiced. However, this largely neglects the contribution of ROS and NO from the tumor microenvironment commonly reported (i.e. chronic inflammatory signaling from TAMs). To date, assaying the amount of SNO in human patient tissue has not yet been described. Additionally, we are experimentally unable to elucidate redox effect on signaling pathways in a clear and mechanistic way. There are, however, several iNOS and ROS modified transgenic mouse models available, i.e. SOD G93A (Henriques, Pitzer et al.), constitutive iNOS (Takamura, Kato et al. 1998), and iNOS knockout (dos Santos, Gattas et al.); that could be used to further delineate which NO/ROS species are the most important in different signaling pathways.

Our current experimental protocol did not include examination of FLIP recruitment to the DISC. Although we determined cellular localization of FLIP we did not coimmunoprecipitate other proteins of the DISC or TNFR signaling complex. Such experiments would further highlight the DR-mediated interactions dependent on S-nitrosylation. Additionally, we did not specifically investigate if the FLIP 2CM harbored any structural modifications or changes in protein folding which could potentially account for signaling differences through domain availability and steric hindrance. Supplementary experiments determining iNOS 
localization following DR-stimulation could also be examined as a potential endogenous NO regulator.

Migratory processes in metastasis are multifactorial and complicated; however in our second study, we clearly demonstrate different ROS species effects on migration and invasion in NSCLC cells. Although we determined these results were Cav-1-dependent, we failed to examine the localization of Cav-1 by confocal microscopy to resolve any potential changes in polarization or caveolae formation that ROS may have incurred. Additionally, $\mathrm{PI} 3 \mathrm{~K} /$ Akt signaling has been described for Cav-1-dependent migration reported by us and others [Appendix, Study 3; (Shack, Wang et al. 2003; Chanvorachote, Nimmannit et al. 2009)], we did confirm that Akt phosphorylation was altered. However, we did not utilize any of the widely available pathway inhibitors (i.e. LY294002, wortmannin) to further demonstrate the ROS-dependent effects.

The role of Cav-1 in tumor promotion and suppression has been widely debated and has largely been suggested to be cell type-dependent (Burgermeister, Liscovitch et al. 2008). We found that different ROS species elicited opposing results with the most reactive ROS species, $\mathrm{OH} \cdot$, promoting both migration and invasion. This finding is incredibly important since $\mathrm{OH}$. is generated via radiation (Halpern, Yu et al. 1995) and chemotherapy treatment (Sinha, Katki et al. 1987). TNF- $\alpha$, which is highly abundant in the tumor microenvironment, has also been reported to increase $\mathrm{OH} \cdot($ Yamauchi, Kuriyama et al. 1989). Increases in $\mathrm{OH}$. levels are noted to induce cell death in tumor cells (Ren, Xia et al. 2001) however chemotherapy-resistant breast cancer cell lines 
appeared to be immune to this induction (Sinha, Katki et al. 1987). This could possibly be attributed to the upregulation of anti-apoptotic proteins (i.e. FLIP and clAPs) in the DR pathway that are frequently seen in treatment-resistant cancers. Therefore, tumor cells that have acquired significant apoptotic-resistance that exist in a chronic inflammatory microenvironment could be subjected to high levels of $\mathrm{OH} \cdot($ as well as other ROS and $\mathrm{NO}$ ), leading them to have increased migratory signaling, such as what we report in our second study.

In conclusion, our studies have presented the effects of NO and ROS on two separate pathways frequently implicated in cancer progression. We are certain that redox biology will become increasingly important in determining the mechanisms of disease and hope that further studies will be pursued. The understanding of redox signaling in the context of pathology could help lead to novel therapeutic targets and clinical breakthroughs in cancer, as well as many other diseases. 


\section{References:}

Boonstra, J. and J. A. Post (2004). "Molecular events associated with reactive oxygen species and cell cycle progression in mammalian cells." Gene 337: 1-13.

Bubici, C., S. Papa, et al. (2006). "The NF-kappaB-mediated control of ROS and JNK signaling." Histol Histopathol 21(1): 69-80.

Burgermeister, E., M. Liscovitch, et al. (2008). "Caveats of caveolin-1 in cancer progression." Cancer Lett 268(2): 187-201.

Chanvorachote, P., U. Nimmannit, et al. (2009). "Nitric oxide regulates lung carcinoma cell anoikis through inhibition of ubiquitin-proteasomal degradation of caveolin-1." J Biol Chem 284(41): 28476-84.

Chanvorachote, P., U. Nimmannit, et al. (2005). "Nitric oxide negatively regulates Fas CD95-induced apoptosis through inhibition of ubiquitin-proteasomemediated degradation of FLICE inhibitory protein." J Biol Chem 280(51): 42044-50.

dos Santos, C. C., D. J. Gattas, et al. "Sepsis-induced myocardial depression is associated with transcriptional changes in energy metabolism and contractile related genes: a physiological and gene expression-based approach." Crit Care Med 38(3): 894-902.

Golks, A., D. Brenner, et al. (2006). "The c-FLIP-NH2 terminus (p22-FLIP)

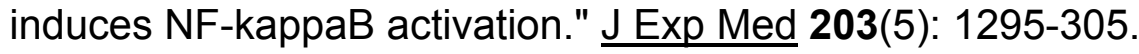

Halpern, H. J., C. Yu, et al. (1995). "In situ detection, by spin trapping, of hydroxyl radical markers produced from ionizing radiation in the tumor of a living mouse." Proc Natl Acad Sci U S A 92(3): 796-800.

Han, D., N. Hanawa, et al. (2006). "Hydrogen peroxide and redox modulation sensitize primary mouse hepatocytes to TNF-induced apoptosis." Free Radic Biol Med 41(4): 627-39. 
Henriques, A., C. Pitzer, et al. "Characterization of a novel SOD-1(G93A) transgenic mouse line with very decelerated disease development." PLoS One 5(11): e15445.

Hess, D. T., A. Matsumoto, et al. (2005). "Protein S-nitrosylation: purview and parameters." Nat Rev Mol Cell Biol 6(2): 150-66.

Igney, F. H. and P. H. Krammer (2002). "Death and anti-death: tumour resistance to apoptosis." Nat Rev Cancer 2(4): 277-88.

Niikura, Y., T. Nonaka, et al. (2002). "Monitoring of caspase-8/FLICE processing and activation upon Fas stimulation with novel antibodies directed against a cleavage site for caspase-8 and its substrate, FLICE-like inhibitory protein (FLIP)." J Biochem 132(1): 53-62.

Pelicano, H., D. Carney, et al. (2004). "ROS stress in cancer cells and therapeutic implications." Drug Resist Updat 7(2): 97-110.

Perry, G., A. K. Raina, et al. (2000). "How important is oxidative damage? Lessons from Alzheimer's disease." Free Radic Biol Med 28(5): 831-4.

Reineke, E. L., Y. Liu, et al. "Promyelocytic leukemia protein controls cell migration in response to hydrogen peroxide and insulin-like growth factor1." J Biol Chem 285(13): 9485-92.

Ren, J. G., H. L. Xia, et al. (2001). "Hydroxyl radical-induced apoptosis in human tumor cells is associated with telomere shortening but not telomerase inhibition and caspase activation." FEBS Lett 488(3): 123-32.

Schrader, M. and H. D. Fahimi (2006). "Peroxisomes and oxidative stress." Biochim Biophys Acta 1763(12): 1755-66.

Shack, S., X. T. Wang, et al. (2003). "Caveolin-induced activation of the phosphatidylinositol 3-kinase/Akt pathway increases arsenite cytotoxicity." Mol Cell Biol 23(7): 2407-14.

Shin, H. S., Z. Zhao, et al. "Synergistic Effect of Tumor Necrosis Factor-Alpha and Hydrogen Peroxide on the Induction of IL-8 Production in Human Intestinal Caco-2 Cells." Inflammation. 
Sinha, B. K., A. G. Katki, et al. (1987). "Adriamycin-stimulated hydroxyl radical formation in human breast tumor cells." Biochem Pharmacol 36(6): 793-6.

Sinha, B. K., A. G. Katki, et al. (1987). "Differential formation of hydroxyl radicals by adriamycin in sensitive and resistant MCF-7 human breast tumor cells: implications for the mechanism of action." Biochemistry 26(13): 3776-81.

Takamura, T., I. Kato, et al. (1998). "Transgenic mice overexpressing type 2 nitric-oxide synthase in pancreatic beta cells develop insulin-dependent diabetes without insulitis." J Biol Chem 273(5): 2493-6.

Wang, J. and J. Yi (2008). "Cancer cell killing via ROS: to increase or decrease, that is the question." Cancer Biol Ther 7(12): 1875-84.

Yamauchi, N., H. Kuriyama, et al. (1989). "Intracellular hydroxyl radical production induced by recombinant human tumor necrosis factor and its implication in the killing of tumor cells in vitro." Cancer Res 49(7): 1671-5.

Yoshizaki, K., T. Fujiki, et al. (2009). "Pro-senescent effect of hydrogen peroxide on cancer cells and its possible application to tumor suppression." Biosci Biotechnol Biochem 73(2): 311-5. 
APPENDIX 


\section{Nitric Oxide Regulates Lung Carcinoma Cell Anoikis through Inhibition of Ubiquitin-Proteasomal Degradation of Caveolin-1*}

Received for publication, July 30, 2009, and in revised form, August 22, 2009 Published, JBC Papers in Press, August 25, 2009, DOI 10.1074/jbc.M109.050864

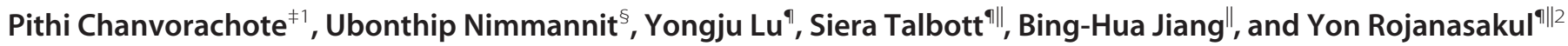

From the ${ }^{\ddagger}$ Department of Physiology and ${ }^{\$}$ Pharmaceutical Technology (International) Program, Chulalongkorn University, Bangkok 10330, Thailand and the "Department of Pharmaceutical Sciences and "Mary Babb Randolph Cancer Center, West Virginia University, Morgantown, West Virginia 26506

Anoikis, a detachment-induced apoptosis, is a principal mechanism of inhibition of tumor cell metastasis. Tumor cells can acquire anoikis resistance which is frequently observed in metastatic lung cancer. This phenomenon becomes an important obstacle of efficient cancer therapy. Recently, signaling mediators such as caveolin-1 (Cav-1) and nitric oxide (NO) have garnered attention in metastasis research; however, their role and the underlying mechanisms of metastasis regulation are largely unknown. Using human lung carcinoma $\mathrm{H} 460$ cells, we show that NO impairs the apoptotic function of the cells after detachment. The NO donors sodium nitroprusside and diethylenetriamine NONOate inhibit detachment-induced apoptosis, whereas the NO inhibitors aminoguanidine and 2-(4-carboxyphenyl) tetramethylimidazoline-1-oxyl-3-oxide promote this effect. Resistance to anoikis in $\mathrm{H} 460$ cells is mediated by Cav-1, which is significantly down-regulated after cell detachment through a non-transcriptional mechanism involving ubiquitinproteasomal degradation. NO inhibits this down-regulation by interfering with Cav-1 ubiquitination through a process that involves protein $S$-nitrosylation, which prevents its proteasomal degradation and induction of anoikis by cell detachment. These findings indicate a novel pathway for NO regulation of Cav-1, which could be a key mechanism of anoikis resistance in tumor cells.

Caveolin-1 (Cav-1), ${ }^{3}$ a 21-24-kDa structural protein component of the plasma membrane microdomains termed caveolae has been shown to function in vesicular trafficking, signal transduction, and cancer progression (1-4). Although up-regulation of this protein normally occurs in a variety of terminally differentiated cells including fibroblasts, adipocytes, smooth muscle

* This work was supported, in whole or in part, by National Institutes of Health Grant R01-HL076340. This work was also supported by Thailand Research Fund and Commission on Higher Education (MRG G5080134).

${ }^{1}$ To whom correspondence may be addressed: Chulalongkorn University, Bangkok 10330, Thailand. E-mail: pithi_chan@yahoo.com.

${ }^{2}$ To whom correspondence may be addressed: WV University, Health Sciences Center, Morgantown, WV 26506. E-mail: yrojan@hsc.wvu.edu.

${ }^{3}$ The abbreviations used are: Cav-1, caveolin-1; SNP, sodium nitroprusside; AG, aminoguanidine; PTIO, 2-(4-carboxyphenyl)-4,4,5,5-tetramethyl-imidazoline-1-oxyl-3-oxide; DTT, dithiothreitol; DAF-DA, diaminofluorescein diacetate; DETA, diethylene triamine; FITC, fluorescein isothiocyanate; $\mathrm{PI}$, propidium iodide; $X T$, sodium 2,3-bis(2-methoxy-4-nitro-5-sulfophenyl)$2 \mathrm{H}$-tetrazolium-5-carboxanilide; $\mathrm{RT}$, reverse transcription; PI3K, phosphatidylinositol 3-kinase. cells, endothelial cells, and epithelial cells (5), Cav-1 is greatly reduced in most oncogenically transformed and cancer cells $(6-10)$. Thus, Cav-1 was first explained to function as a tumor suppressor protein (11-13). In contrast, increasing evidence indicates its role as a tumor and metastatic promoter as overexpression or re-expression of Cav-1 was found in many advanced stage and metastatic cancer cells. Up-regulation of Cav-1 was shown to render Rat1A cells more resistant to apoptosis (14). Moreover, antisense-induced down-regulation of Cav-1 caused human prostate cancer cells more sensitive to apoptosis $(15,16)$. Therefore, the role of Cav-1 in cancer progression remains controversial.

Metastasis is a multistep process composed of cancer cell detachment, migration, extravasation, and adhesion of the detached cells to other target sites. A key mechanism in the regulation of metastasis is anoikis or detachment-induced apoptosis. Previous studies have shown that Cav-1 acts as a negative regulator of anoikis $(17,18)$, and its elevated expression in lung carcinoma is closely associated with the increased metastasis capacity and poor survival of the patients (19). Likewise, elevated NO and NO synthase levels have been associated in many human metastatic cancers including the lung (20-24), breast (25), colon (26), and cancers of the central nervous system (27). However, the role of NO and its mechanism of metastasis regulation in association with $\mathrm{Cav}-1$ are not well understood. Because resistance to anoikis is a key step in metastasis development and because Cav-1 has been implicated in this process, we investigated the potential regulation of Cav-1 by $\mathrm{NO}$ and studied its role in anoikis of human lung carcinoma cells.

NO has been reported to have both pro- and anti-apoptotic effect on cells, depending on a variety of factors, including cell type, cellular redox status, and the flux and dose of local NO (28, 29). In human lung carcinoma cells, we previously reported that NO plays a suppressive role in apoptosis induced by a variety of agents, including Fas death ligand (30), chemotherapeutic agent cisplatin (31), and the metal carcinogen chromium (32). However, the role of $\mathrm{NO}$ in cell anoikis and its potential regulation of Cav-1 have not been well investigated. Using molecular and pharmacological approaches, we report here that NO plays an important role in Cav-1 regulation and anoikis function of human lung cancer $\mathrm{H} 460$ cells. We also demonstrate for the first time that Cav-1 is down-regulated during cell anoikis through the ubiquitin-proteasomal degradation pathway and 
that NO regulates this process by inducing $S$-nitrosylation of the protein which inhibits its ubiquitination and proteasomal degradation. Thus, our study reveals the existence of a novel mechanism of anoikis regulation, which might be exploited in metastasis and cancer therapy.

\section{MATERIALS AND METHODS}

Cells and Reagents-Human lung epithelial NCI-H460 cells were obtained from the American Type Culture Collection (Manassas, VA). The cells were cultured in RPMI 1640 medium supplemented with $5 \%$ fetal bovine serum, $2 \mathrm{~mm}$ L-glutamine, and 100 units/ml penicillin and streptomycin. Cell cultures were maintained in a humidified atmosphere of $5 \% \mathrm{CO}_{2}$ at $37^{\circ} \mathrm{C}$. Cells were passaged at preconfluent densities using a solution containing $0.05 \%$ trypsin and $0.5 \mathrm{~mm}$ EDTA. The NO donors sodium nitroprusside (SNP) and diethylene triamine (DETA) NONOate and the NO inhibitors 2-(4-carboxyphenyl)-4,4,5,5-tetramethylimidazoline-1-oxyl3 -oxide (PTIO) and aminoguanidine (AG) were obtained from Sigma. These NO modulators are water-soluble and were prepared in a sterile culture medium before use. Diaminofluorescein diacetate (DAF-DA) and Hoechst 33342 were obtained from Molecular Probes, Inc. (Eugene, OR). Monoclonal antibody against Cav-1 and protein A-agarose were from Santa Cruz Biotechnology (Santa Cruz, CA). Antibodies against Akt and phospho-Akt Ser-473 and Thr-308 were from Cell Signaling Technology, Inc. (Beverly, MA). Antibodies for ubiquitin, $S$-nitrosocysteine, $\beta$-actin, and peroxidase-conjugated secondary antibodies were from Sigma. The transfecting agent Lipofectamine 2000 was from Invitrogen. All other chemicals and reagents including annexin V-fluorescein isothiocyanate (FITC), propidium iodide (PI), dithiothreitol (DTT), lactacystin, and LY294002 were from Sigma.

NO Detection-Cellular NO production was determined by flow cytometry using DAF-DA as a fluorescent probe and by Griess assay, which measures the stable nitrite byproduct of NO in the culture medium. After detachment, cells $\left(1 \times 10^{6} / \mathrm{ml}\right)$ were collected and incubated with $10 \mu \mathrm{M}$ DAF-DA for $30 \mathrm{~min}$ at $37^{\circ} \mathrm{C}$. The cells were then washed, resuspended in phosphatebuffered saline, and analyzed for fluorescence intensity using FACSCaliber (BD Biosciences). Signals were obtained using a 488-nm excitation beam and a 538-nm band-pass filter. In some experiments cells were visualized for fluorescence intensity using a fluorescence microscope (Carl Zeiss Axiovert, Göttingen, Germany). For the Griess assay, cell supernatants were collected, and aliquots $(100 \mu \mathrm{l})$ were mixed with $100 \mu \mathrm{l}$ of Griess reagent ( $1 \%$ sulfanilamide, $0.1 \%$ naphthyl ethylenediamine dihydrochloride, $2 \%$ phosphoric acid) in a 96 -well plate. After incubation for $10 \mathrm{~min}$ at $25^{\circ} \mathrm{C}$, the absorbance at $550 \mathrm{~nm}$ was measured on a microplate reader.

Plasmid and Transfection-Caveolin-1 plasmid (pEX_Cav1-YFP) was obtained from the American Type Culture Collection (Manassas, VA). Stable transfectant of Cav-1 was generated by culturing $\mathrm{H} 460$ cells in a 6-well plate until they reached $60 \%$ confluence. One microgram of cytomegalovirus-neo vector and $15 \mu \mathrm{l}$ of Lipofectamine reagent with $2 \mu \mathrm{g}$ of Cav-1 or control pcDNA3 plasmid were used to transfect the cells in the absence of serum. After $12 \mathrm{~h}$ the medium was replaced with culture medium containing 5\% fetal bovine serum. Approximately $36 \mathrm{~h}$ after the beginning of the transfection, the cells were digested with $0.03 \%$ trypsin, and the cell suspensions were plated onto $75-\mathrm{ml}$ culture flasks and cultured for $24-28$ days with G418 selection $(600 \mu \mathrm{g} / \mathrm{ml})$. The pooled stable transfectant was identified by Western blot analysis of Cav-1 and was cultured in G418-free RPMI 1640 medium for at least two passages before each experiment.

Anoikis Assays-Adherent H460 cells in culture were trypsinized into a single cell suspension and then seeded in 12-well tissue culture plates coated with $200 \mu \mathrm{l}(6 \mathrm{mg} / \mathrm{ml}$ in $95 \%$ ethanol) of poly-2-hydroxyethylmethacrylate (poly-HEMA; Sigma) at the density of $1 \times 10^{5}$ cells $/ \mathrm{ml}$. Suspended cells were incubated at $37^{\circ} \mathrm{C}$ for various times up to $24 \mathrm{~h}$. Cells were then harvested, washed, and stained with annexin V-FITC and analyzed for fluorescence intensity by flow cytometry and fluorescence microscopy. For Hoechst 33342 apoptosis assay, cells were incubated with $10 \mu \mathrm{m}$ the Hoechst dye for $30 \mathrm{~min}$ at $37^{\circ} \mathrm{C}$, and the apoptotic cells having intensely condensed chromatin and/or fragmented nuclei were visualized under a fluorescence microscope. For cell survival assay, cells were similarly treated, harvested, washed, and incubated with $20 \mu \mathrm{m}$ sodium 2,3-bis(2methoxy-4-nitro-5-sulfophenyl)-2H-tetrazolium-5-carboxanilide (XTT) for $4 \mathrm{~h}$ at $37^{\circ} \mathrm{C}$. Optical density was then determined by V-max photometer (Molecular Devices, Inc., Menlo Park, CA) at a wavelength of $450 \mathrm{~nm}$.

Western Blot Analysis-Cell extracts were performed by incubating the cells in lysis buffer containing $20 \mathrm{~mm}$ Tris- $\mathrm{HCl}$, $\mathrm{pH} 7.5,1 \%$ Triton X-100, $150 \mathrm{~mm}$ sodium chloride, $10 \%$ glycerol, 1 mm sodium orthovanadate, $50 \mathrm{~mm}$ sodium fluoride, 100 $\mathrm{mm}$ phenylmethylsulfonyl fluoride, and a protease inhibitor mixture (Roche Applied Science) for $30 \mathrm{~min}$ on ice. Cell lysates were collected and assayed for protein content using the Bradford method (Bio-Rad). Equal amount of proteins per sample $(40 \mu \mathrm{g})$ were resolved on a 10\% SDS-polyacrylamide gel electrophoresis and transferred onto $0.45-\mu \mathrm{m}$ nitrocellulose membranes (Pierce). The transferred membranes were blocked for $1 \mathrm{~h}$ in 5\% nonfat dry milk in Tris-buffered saline/Tween 20 (25 mM Tris- $\mathrm{HCl}, \mathrm{pH} 7.4,125 \mathrm{~mm} \mathrm{NaCl}$, and $0.05 \%$ Tween 20) and incubated with the appropriate primary antibodies at $4{ }^{\circ} \mathrm{C}$ overnight. Membranes were washed 3 times with Tris-buffered saline, Tween 20 for $10 \mathrm{~min}$ and incubated with peroxidaselabeled secondary antibodies for $1 \mathrm{~h}$ at room temperature. The immune complexes were detected by chemiluminescence (Supersignal West Pico; Pierce) and quantified by imaging densitometry using analyst/PC densitometry software (Bio-Rad).

Immunoprecipitation-Cells were washed after treatments and lysed in lysis buffer for $30 \mathrm{~min}$ on ice. Cell lysates were collected and determined for protein content. Equal amounts of proteins per sample $(60 \mu \mathrm{g})$ were immunoprecipitated with anti-Cav-1 antibody for $6 \mathrm{~h}$ at $4{ }^{\circ} \mathrm{C}$. The immune complexes were washed with 30 volumes of lysis buffer, resuspended in $2 \times$ Laemmli sample buffer, and boiled at $95^{\circ} \mathrm{C}$ for $5 \mathrm{~min}$. The immune complexes were separated by $10 \%$ SDS-PAGE and analyzed by Western blotting as described above.

Measurements of Cav-1 S-Nitrosylation-Cells were lysed and immunoprecipitated with anti-Cav-1 antibody as described above. The immunoprecipitated protein was analyzed 
for $S$-nitrosylation by Western blot using anti-S-nitrosocysteine antibody and by fluorometric measurements as previously described (32). Briefly, immunoprecipitates were incubated with $200 \mu \mathrm{M} \mathrm{HgCl}$ and $200 \mu \mathrm{M}$ diamino naphthalene in $500 \mu \mathrm{l}$ of phosphate-buffered saline for $0.5 \mathrm{~h}$ at room temperature followed by the addition of $1 \mathrm{~m} \mathrm{NaOH}$. The fluorescent triazole product generated from the reaction between diamino naphthalene and NO released from $S$-nitrosylated Cav-1 was quantified by fluorometry at the excitation and emission wavelengths of 375 and $450 \mathrm{~nm}$, respectively.

Reverse Transcription-PCR-Total RNA was extracted with Trizol (Invitrogen), and reverse transcription-PCR was performed with Access RT-PCR System (Promega, Madison, WI) according to the manufacturer's instructions. Sequences of the PCR primers were: Cav-1 forward, $5^{\prime}$-CGTAGACTCGGAGGGACATC-3', and reverse, 5'-TTTCGTCACAGTGAAGGTGG-3'; for glyceraldehyde-3-phosphate dehydrogenase, forward, 5' -GCTGAGAACGGGAAGCTTGT-3', and reverse, 5'-GCCAGGGGTGCTAAGCAG-3'. Reaction products were analyzed after 30 amplification cycles, each of which involved consecutive 1 -min steps at 94,55 , and $72{ }^{\circ} \mathrm{C}$. The PCR products were electrophoresed in a $1.5 \%$ agarose gel, stained with ethidium bromide, and photographed.

The results obtained by conventional RT-PCR were verified by quantitative real-time PCR. One microgram of Trizolextracted RNA was reverse-transcribed in a $100-\mu$ l reaction mixture containing $500 \mu \mathrm{M}$ dNTP, 125 units of MultiScribe Reverse Transcriptase (Applied Biosystems, Foster City, CA), 40 units of RNase inhibitor, $2.5 \mu \mathrm{M}$ oligo(dT), $1 \times$ TaqMan reverse transcriptase buffer, and $5 \mathrm{mM} \mathrm{MgCl}_{2}$ at $48{ }^{\circ} \mathrm{C}$ for $40 \mathrm{~min}$. The primers used in this study were designed using Primer Express software (Applied Biosystems): Cav-1 (\#AI878826) forward 5'-CGAGAAGCAAGTGTACGACGC3', and reverse 5' -ACCACGTCATCGTTGAGGTG-3'; glyceraldehyde-3-phosphate dehydrogenase forward, 5'-GAAGGTGAAGGTCGGAGTC-3', and reverse 5'-GAAGATGGTGATGGGATTTC-3'. Amplification was performed at the following cycling conditions: $95^{\circ} \mathrm{C}$ for $10 \mathrm{~min}$ followed by 40 cycles at $95^{\circ} \mathrm{C}$ for $15 \mathrm{~s}$ and $60^{\circ} \mathrm{C}$ for $1 \mathrm{~min}$. A SYBR Green PCR Master Mix (Applied Biosystems) was used with $1 \mathrm{ng}$ of cDNA and with 100-400 nM primers. A negative control without any cDNA template was run with every assay. All PCR reactions were performed by using ABI PRISM 7900 Sequence Detection System (Applied Biosystems). Relative mRNA levels were determined by using the comparative $\mathrm{C}_{\mathrm{T}}$ (threshold cycle) method (33), where the caveolin-1 target is normalized to the control and compared with a reference sample (assigned a relative value of 1 ) by the equation: $2^{-\Delta \Delta C T}$.

Immunofluorescence-Cells $\left(0.5 \times 10^{6} /\right.$ well $)$ were seeded in 6-well poly-HEMA-coated plates and treated with NO modulators as described under "Results." After treatment, the cells were fixed in $3.7 \%$ formaldehyde for $10 \mathrm{~min}$ at room temperature and then permeabilized and blocked in a solution containing $0.5 \%$ saponin, $1 \%$ bovine serum, and $1.5 \%$ goat serum for 30 min. After primary antibody incubation with Cav-1 monoclonal antibody (BD Biosciences) at 1:100 dilution for $1 \mathrm{~h}$, cells were washed and incubated with Alexa Fluor 488-conjugated secondary antibody (Invitrogen) for $30 \mathrm{~min}$. Cell nucleus was stained with ToPro-3 (Invitrogen), and the actin cytoskeleton was stained with Alexa Fluor 546-conjugated phalloidin (Invitrogen). Cells were cytospun onto a glass slide and mounted using the anti-fade reagent Fluoromont-G (Southern Biotech, Birmingham, AL). Images were acquired by confocal laser scanning microscopy (Zeiss LSM 510).

Statistical Analysis-Data were represented as the means \pm S.D. from three or more independent experiments. Statistical analysis was performed by Student's $t$ test at a significance level of $p<0.05$.

\section{RESULTS}

Nitric Oxide Inhibits Detachment-induced Apoptosis of H460 Cells-NO has been shown to play an important role in the regulation of cancer cell metastasis; however, the underlying mechanism of this regulation is unclear. To test whether NO might regulate this process by inhibiting detachment-induced apoptosis or anoikis, which is a crucial step in the metastasis of cancer cells, we first investigated anoikis of human lung cancer H460 cells in response to various specific NO donors and inhibitors. Anoikis was induced by detaching the cells and incubating them in attachment-resistant poly-HEMA-coated plates for various times and analyzed for cell viability by XTT assay. Fig. $1 A$ shows that detachment of the cells caused a time-dependent decrease in cell viability with $\sim 55$ and $15 \%$ of the cells remaining viable after 6 and 12 h, respectively. Analysis of cell apoptosis by flow cytometry using FITC-labeled annexin $\mathrm{V}$ antibody shows a significant increase in annexin $\mathrm{V}$-associated cellular fluorescence as early as $6 \mathrm{~h}$ after the detachment and reached a maximum at about $18 \mathrm{~h}$ (Fig. $1 B$ ). In contrast, analysis of cell necrosis using PI as a probe shows no significant increase in the PI signal over a 24-h period. These results suggest that apoptosis is the primary mode of cell death after detachment of $\mathrm{H} 460$ cells. Morphologic analysis of apoptotic cell death by fluorescence microscopy using Hoechst 33342 and annexin V-FITC further confirms the results (Fig. $1 E$ ).

To investigate the role of NO in detachment-induced apoptosis, detached H460 cells were treated with various concentrations of NO donors and inhibitors, and their effect on cell survival was determined by XTT assay. Fig. $1 C$ shows that treatment of the cells with NO donor, SNP, or DETA NONOate caused a dose-dependent decrease in cell death, whereas treatment of the cells with NO inhibitor, AG, or PTIO had an opposite effect. Analysis of cell apoptosis by annexin V-FITC and Hoechst 33342 assays similarly shows the inhibitory and promoting effect of the NO donors and inhibitors, respectively, on detachment-induced cell death (Fig. 1, D and E). The NO donors and inhibitors, when used at the indicated concentrations, had no significant effect on cell necrosis as determined by PI assay (Fig. 1D).

Effect of NO Modulators on Cellular NO Level-To provide a relationship between cell death and NO modulation induced by the test agents, we analyzed cellular NO levels in response to various NO modulator treatments by colorimetric Griess assay and by flow cytometric and microscopic assays using DAF-DA as a fluorescent probe for NO. Fig. $2 A$ shows the result of the Griess assay which measures the stable nitrite breakdown product of NO. Both NO inhibitors AG and PTIO significantly 
A
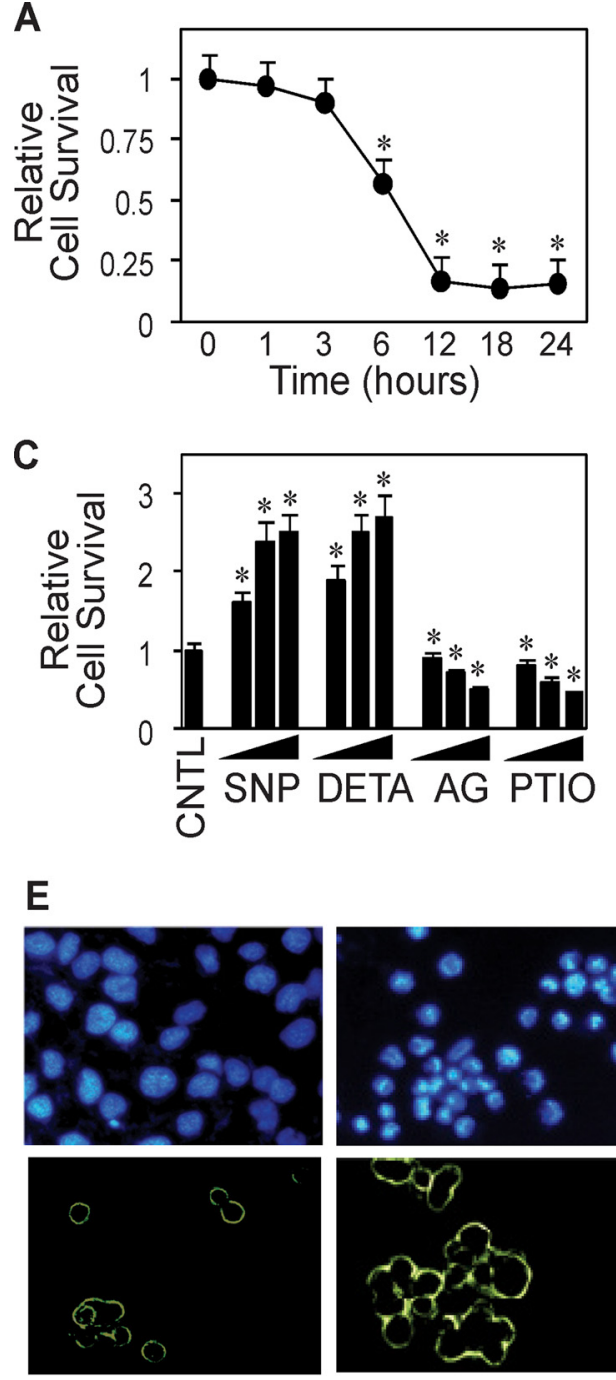

Control

$(0 \mathrm{~h})$
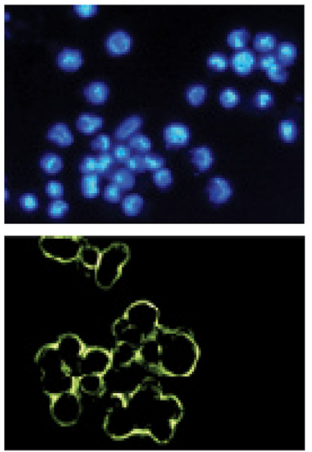

Control

(12 h)
B

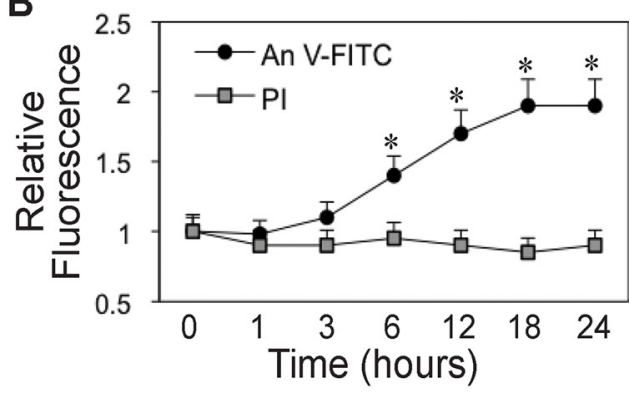

D

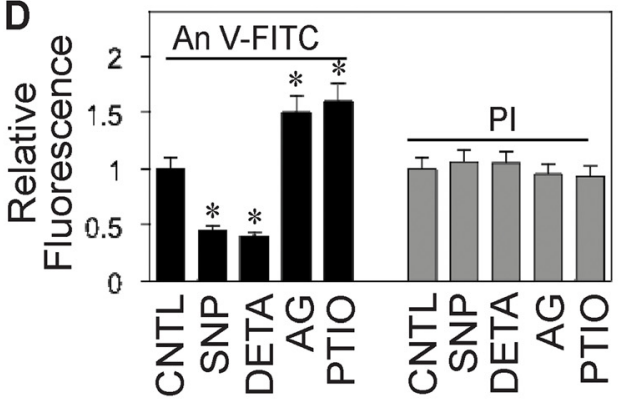

and evaluated their effect on detachment-induced cell death. Transfected cells were detached, suspended in poly-HEMA-coated plates, and analyzed for cell survival at various times by XTT assay. Fig. $3 A$ shows that Cav-1-transfected cells exhibited resistance to detachment-induced cell death as compared with control-transfected cells. Western blot analysis of Cav-1 expression in the transfected cells shows an increased expression of Cav-1 protein in the Cav-1-transfected cells compared with controltransfected cells (Fig. 3B). These results indicate the role of $\mathrm{Cav}-1$ as a negative regulator of detachmentinduced cell death in lung epithelial H460 cells. Because Cav-1 has been shown to regulate cell death and survival through a PI3K/Akt-dependent mechanism $(16,35,36)$, we tested the effect of Cav-1 overexpression on Akt activity and examined its effect on detachment-induced cell death. Our results show that Cav-1 overexpression induced Akt activation as indicated by the increased phosphorylation of Akt (Thr-308 and Ser-473), whereas it had no effect on total Akt level (Fig. $3 C)$. The induction of Akt phosphorylation was inhibited by the PI3K inhibitor LY294002, suggesting that this induction was mediated through the PI3K pathway. Analysis of apoptosis shows that Cav-1 overexpression decreased detachmentinduced cell death and that this effect was inhibited by the PI3K inhibitor LY294002 (Fig. 3D). These results suggest that Cav-1 exerts its anti-apoptotic effect during cell detachment through a mechanism that is dependent on PI3K/Akt activation.

FGURE 1. Detachment-induced apoptosis and its inhibition by NO. $A$, effect of cell detachment survival determined by XTT assay. Lung epithelial $\mathrm{H} 460$ cells were detached as described under "Materials and Methods" and suspended in HEMA-coated plates for various times (0-24 h). $B$, effect of cell detachment on apoptosis and necrosis determined by flow cytometry using annexin V-FITC (An V-FITC) and PI assays. C, effect of NO modulators on detachment-induced cell death. Detached cells were treated with various concentrations of NO donor, SNP $(10,50,100 \mu \mathrm{M})$, or DETA NONOate $(10,50,100 \mu \mathrm{M})$ or with NO inhibitor, AG $(100,200,300$ $\mu \mathrm{M})$, or PTIO $(10,50,100 \mu \mathrm{M})$ for $12 \mathrm{~h}$. Cell survival was then determined by XTT assay. CNTL, control. D, effects of NO modulators on detachment-induced apoptosis and necrosis. Detached cells were treated with SNP (50 $\mu \mathrm{M})$, DETA NONOate $(50 \mu \mathrm{M})$, AG $(300 \mu \mathrm{M})$, or PTIO $(50 \mu \mathrm{M})$ for $12 \mathrm{~h}$, and cell apoptosis and necrosis were determined as described above. E, upper panel, effect of NO modulators on detachment-induced apoptosis determined by Hoechst 33342 nuclear fluorescence staining. Lower panel, effect of NO modulators on detachment-induced apoptosis determined by annexin V-FITC fluorescence microscopy. Data are the mean \pm S.D. $(n=3) .^{*}, p<0.05$ versus non-treated control.

inhibited cellular nitrite production, whereas the NO donors SNP and DETA NONOate increased the production as compared with non-treated control. These results were confirmed by flow cytometric and microscopic assays of $\mathrm{NO}$ (Fig. 2, $B$ and $C$ ), which show the induction and inhibition of cellular NO levels by the NO donor SNP and NO inhibitor PTIO, respectively.

Cav-1 Overexpression Renders H460 Cells Resistant to Detachment-induced Apoptosis-The role of Cav-1 in the regulation of cancer cell anoikis is unclear. We studied this role by stably transfecting $\mathrm{H} 460$ cells with Cav-1 or control plasmid
Cav-1 Overexpression Alters Cell Growth and Morphology of H460 Cells-Fig. $3 E$ shows that under a normal growth condition that allows cell attachment, Cav-1-overexpressing cells exhibited an increased growth rate over control-transfected cells. The lag phase before cell growth was significantly reduced in Cav-1-overexpressing cells. As compared with control-transfected cells, which grew as an epithelial monolayer, Cav-1-overexpressing cells formed cell mounds and grew as multilayer epithelial cells (Fig. 3F). This multilayer growth pattern is consistent with the increased growth rate of Cav-1-overexpressing cells. These results suggest that 

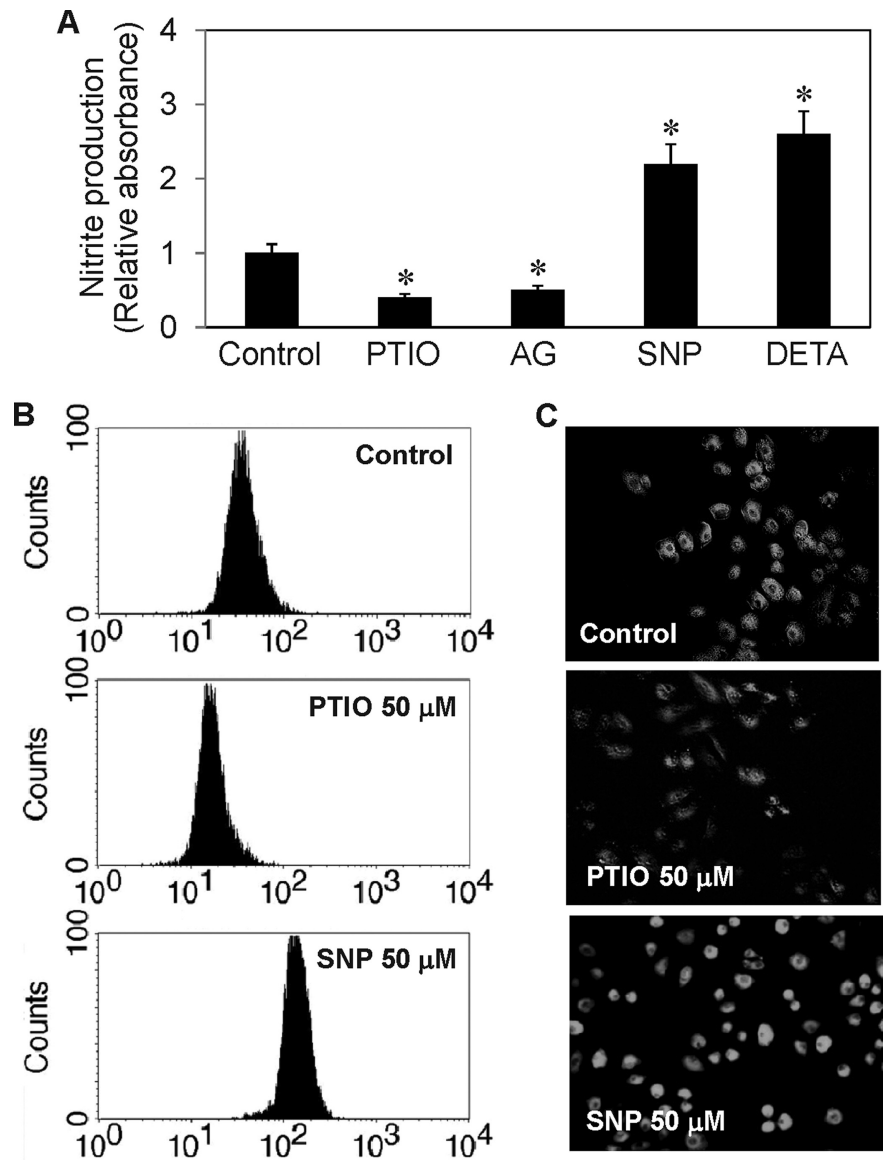

FIGURE 2. Effect of NO modulators on cellular NO and nitrite levels. H460 cells were detached and either left untreated or treated with SNP $(50 \mu \mathrm{M})$, DETA NONOate $(50 \mu \mathrm{M})$, AG $(300 \mu \mathrm{M})$, or PTIO $(50 \mu \mathrm{M})$ for $2 \mathrm{~h}$. A, nitrite production determined by the Griess assay. $B$ and $C$, NO production determined by flow cytometry and fluorescence microscopy using DAF-DA as a probe. Data are the mean \pm S.D. $(n=3) .{ }^{*}, p<0.05$ versus non-treated control.

Cav-1 may function as a tumor promoter by enhancing cell growth.

Detachment Induces Cav-1 Down-regulation through a Nontranscriptional Proteasome-dependent Mechanism-Having demonstrated the role of Cav-1 as a negative regulator of cell anoikis in $\mathrm{H} 460$ cells, we next investigated the expression profile of Cav-1 after cell detachment. Detached cells were suspended in attachment-resistant plates for various times and analyzed for Cav-1 protein and mRNA expression by Western blotting and RT-PCR, respectively. Fig. $4 A$ shows that Cav-1 protein levels were significantly reduced in cells after detachment in a time-dependent manner. The reduction was strongly inhibited by lactacystin, a specific proteasome inhibitor, suggesting that detachment-induced Cav-1 down-regulation was mediated through proteasomal degradation. This result was confirmed by the observation that another proteasome inhibitor, MG132, also inhibited the decrease in Cav-1 protein expression (data not shown). Analysis of Cav-1 mRNA levels by RT-PCR shows that Cav-1 transcripts were relatively unchanged after cell detachment (Fig. 4B), whereas the protein levels were substantially reduced. The RT-PCR result was confirmed by quantitative real-time RT-PCR, which shows no significant changes in the Cav-1 mRNA level after cell detachment (Fig. 4C). Thus, cell detachment appears to cause Cav-1 protein
A

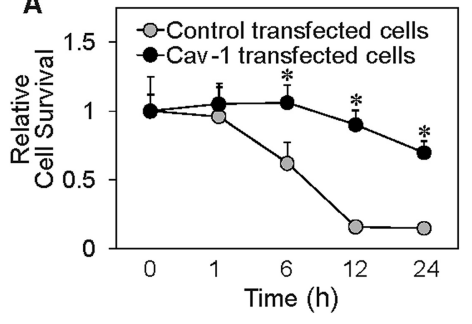

C
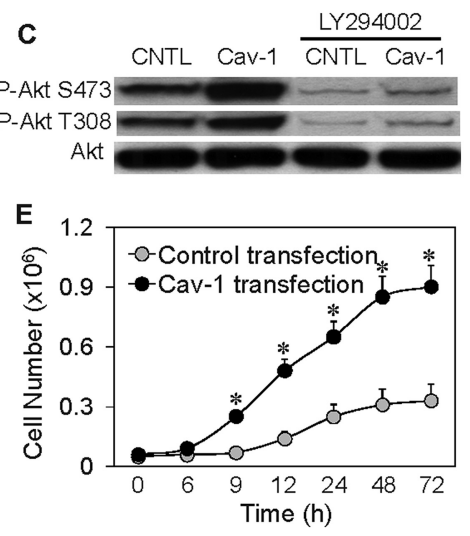

B
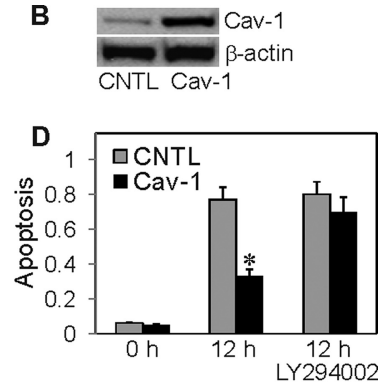

$\mathbf{F}$

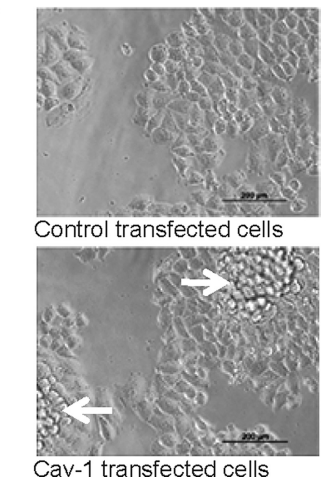

FIGURE 3. Cav-1 overexpression increases cell death resistance and Akt phosphorylation, alters growth pattern, and increases growth rate. A, H460 cells were stably transfected with Cav- 1 or control plasmid as described under "Materials and Methods." Transfected cells were grown in culture medium and analyzed for cell survival at various times after detachment using XTT assay. Attached cells showed no significant apoptosis during the test period of $24 \mathrm{~h}$ (data not shown). B, Western blot analysis of Cav-1 expression in control and Cav-1-transfected cells. Cell extracts were prepared and separated on $10 \%$ polyacrylamide-SDS gels, transferred, and probed with Cav- 1 antibody. $\beta$-Actin was used as a loading control. CNTL, control. $C$, effect of Cav-1 overexpression on Akt phosphorylation. Transfected cells were detached and incubated in HEMA-coated plates for $12 \mathrm{~h}$ in the presence or absence of LY294002 (10 $\mu \mathrm{M})$. Cell lysates were prepared and analyzed for Akt phosphorylation by Western blotting. Blots were probed with antibodies specific to phospho-Akt (Ser-473 and Thr-308) and Akt. D, apoptosis of the treated cells was analyzed by Hoechst 33342 assay and expressed as the ratio of apoptotic nuclei to total nuclei. $E$, effect of Cav-1 overexpression on cell proliferation. Cav-1 and control-transfected cells were grown in normal tissue culture plates and analyzed for cell proliferation at various times by hemocytometry. $F$, morphology of control and Cav-1-transfected cells in culture. Data are the mean \pm S.D. $(n=3) .{ }^{*}, p<0.05$ versus vector-transfected control.

reduction through a transcription-independent mechanism. These results along with subsequent data showing the effect of cell detachment on Cav-1 ubiquitination support the role of ubiquitin-proteasomal degradation as an important mechanism of Cav-1 down-regulation induced by cell detachment.

Nitric Oxide Prevents Detachment-induced Cav-1 Down-regulation-We further investigated the potential regulation of Cav-1 by NO. Cells were detached and suspended in HEMAcoated plates in the presence or absence of NO donors and inhibitors. Cav-1 protein expression was then determined by Western blotting. Fig. $4 \mathrm{D}$ shows that the NO donors SNP and DETA NONOate strongly inhibited detachment-induced Cav-1 down-regulation at the concentrations shown to induce an increase in cellular NO levels (Fig. 2). In contrast, the NO inhibitors AG and PTIO promoted this down-regulation (Fig. $4 D)$. These results were confirmed by confocal immunofluorescence microscopy which shows that Cav-1 fluorescence intensity was reduced after cell detachment and that the NO donor SNP was able to inhibit this reduction, whereas the NO inhib- 
A
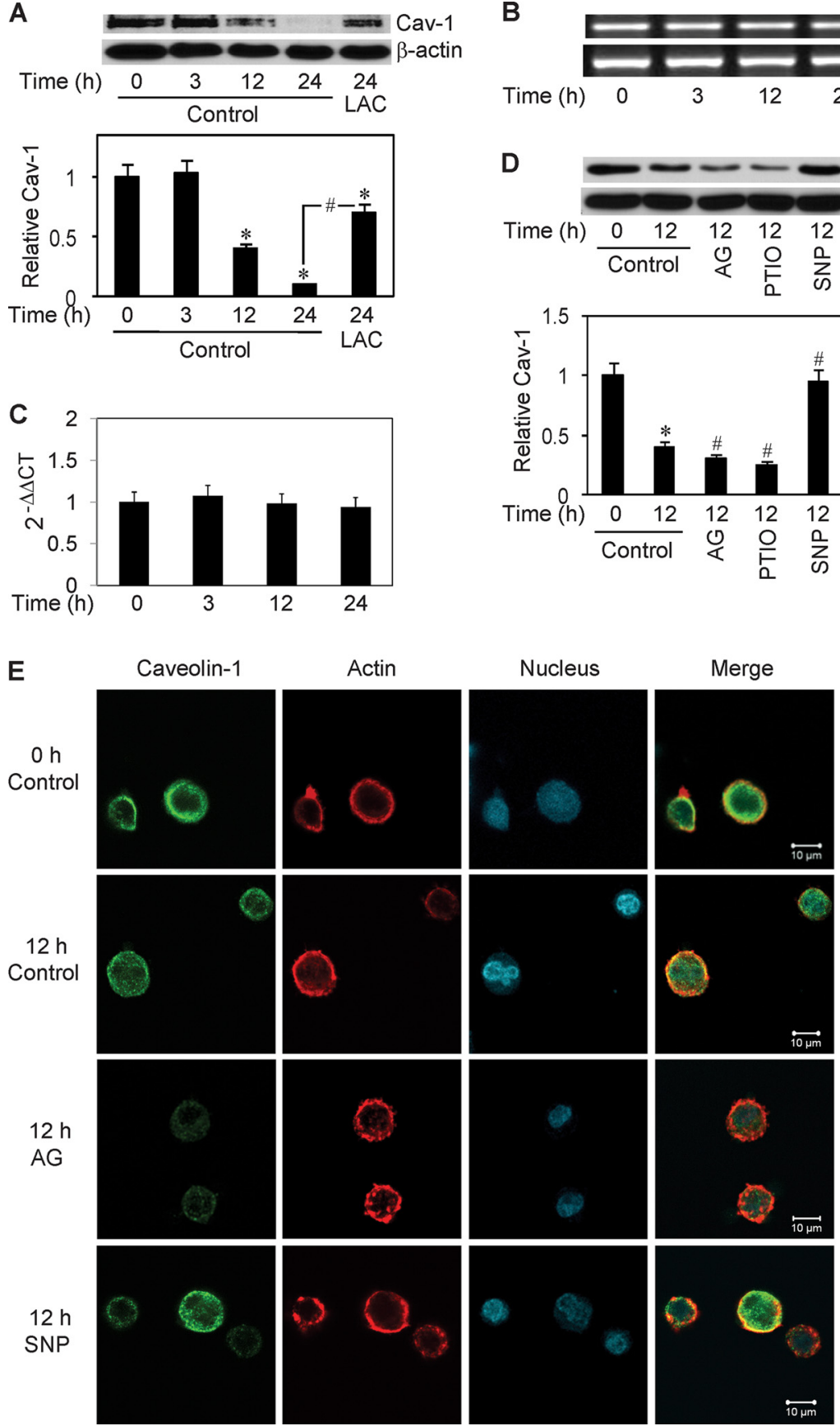

FIGURE 4. Effect of cell detachment on Cav-1 expression and its regulation by NO. $A$, H460 cells were detached and seeded in HEMA-coated plates for various times (0-24 h) in the presence or absence of lactacystin (LAC) $(10 \mu \mathrm{M})$. Cells extracts were prepared and analyzed for Cav-1 protein expression by Western blotting. Blots were reprobed with $\beta$-actin antibody to confirm equal loading of samples. The immunoblot signals were quantified by densitometry, and mean data from independent experiments (one of which is shown here) were normalized to the results in control cells at 0 h. B, RT-PCR analysis of Cav-1 and glyceraldehyde-3-phosphate dehydrogenase $(G A P D H)$ mRNA expression at various times (0-24 h) after cell detachment. $C$, relative Cav- 1 mRNA expression determined by quantitative real-time $P C R$. The relative mRNA expression was determined by using the comparative $C_{T}$ method as described under "Materials and Methods." $D$, detached cells were treated with NO inhibitor, AG $(300 \mu \mathrm{M})$ or PTIO $(50 \mu \mathrm{M})$, or with NO donor, SNP $(50 \mu \mathrm{M})$, or DETA NONOate $(50 \mu \mathrm{M})$ for $12 \mathrm{~h}$, after which they were analyzed for Cav-1 expression by Western blotting. $E$, detached cells were either left untreated or treated with SNP $(50 \mu \mathrm{M})$ or AG $(300 \mu \mathrm{M})$ for $12 \mathrm{~h}$ and analyzed for Cav-1 by immunofluorescence confocal microscopy. Cells were also stained with Alexa Fluor 546-conjugated phalloidin and ToPro-3 to aid visualization of actin cytoskeleton and nucleus. Data are the mean \pm S.D. $(n=3) .{ }^{*}, p<0.05$ versus attached cell control; $\#, p<0.05$ versus the indicated control or 12-h detached cell control.

itor AG further reduced the signal intensity (Fig. 4E). Although these results are non-quantitative, they support the Western blot data and provide additional evidence for the suppressive role of NO in detachment-induced Cav-1 down-regulation. Analysis of the fluorescence pattern in the treated and control cells shows no apparent changes in the cellular localization of Cav-1, which is largely membrane-bound.

Detachment Induces Cav-1 Ubiquitination and Its Inhibition by NOThe observation that detachmentinduced Cav-1 down-regulation was inhibited by proteasome inhibitors suggests that this down-regulation could be mediated by protein ubiquitination and subsequent degradation by the proteasome. Because ubiquitination of Cav-1 has not been reported, we examined whether cell detachment could induce Cav-1 ubiquitination and whether or not this process is regulated by NO. Cells were detached and suspended in HEMA-coated plates in the presence or absence of NO donors for various times. Cell lysates were prepared and immunoprecipitated with anti-Cav-1 antibody, and the resulting immune complexes were analyzed for ubiquitin by Western blotting. Fig. 5A shows that Cav-1 was rapidly ubiquitinated as early as $1 \mathrm{~h}$ after cell detachment and peaked at about $3 \mathrm{~h}$. The NO donors SNP and DETA NONOate strongly inhibited this ubiquitination, suggesting that NOmediated inhibition of protein ubiquitination could be a key mechanism of Cav-1 stabilization by NO, and our subsequent study supports this notion.

NO-mediated S-Nitrosylation of Cav-1 as a Potential Mechanism of Protein Stability Regulation-Previous studies have shown that NO induced $S$-nitrosylation of some apoptosis regulatory proteins, such as c-FLIP and Bcl-2, and prevented their ubiquitination and degradation by the proteasome $(30-32)$. To test whether $S$-nitrosylation could be involved in the regulation of Cav-1 by NO, we performed immunoprecipitation experiments examining the effect of $\mathrm{NO}$ on Cav-1 $S$-nitrosylation and protein expression. The results show that treatment of the cells with NO donors, SNP, and DETA NONOate induced $S$-nitrosylation of Cav-1 as determined by 
A

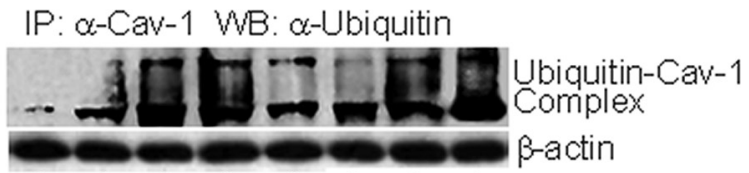

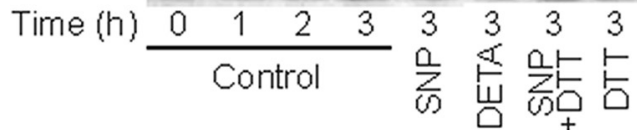

B

IP: $\alpha-$ Cav-1 WB: $\alpha-S-N i t r o s a c y s t e i n$
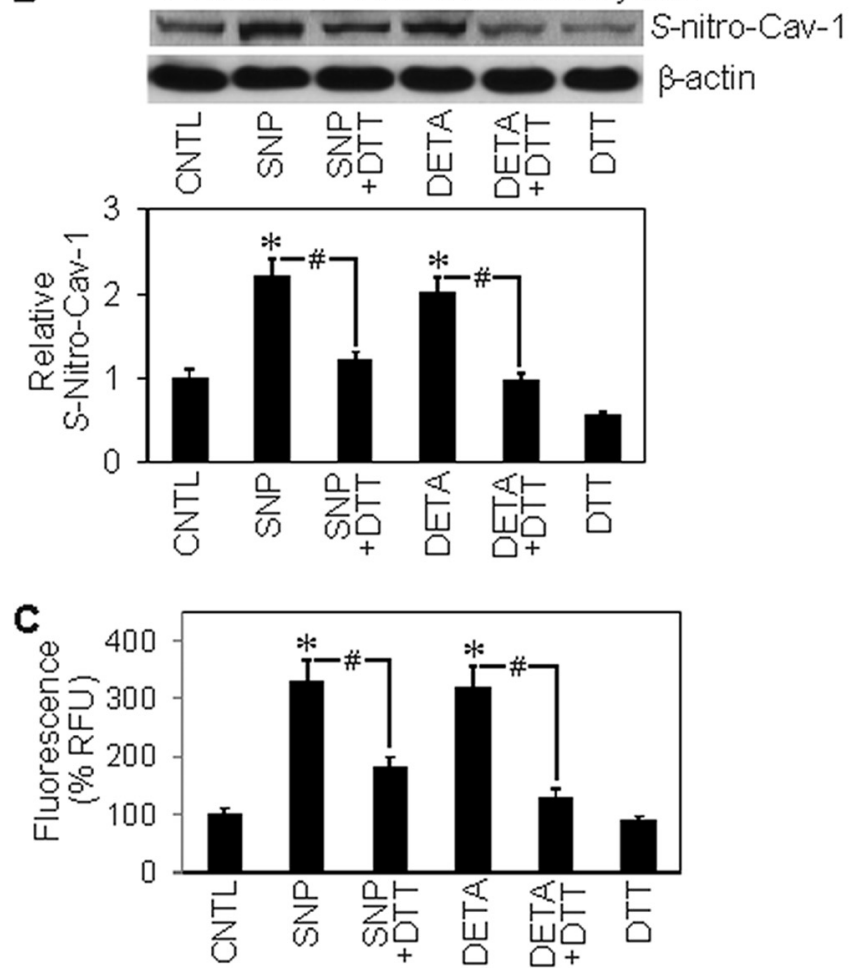

FIGURE 5. Effects of NO modulators on Cav-1 ubiquitination and S-nitrosylation. $A, \mathrm{H} 460$ cells were detached and either left untreated or treated with SNP $(50 \mu \mathrm{M})$ or DETA NONOate $(50 \mu \mathrm{M})$ in the presence or absence of DTT (1 $\mathrm{mm}$ ) in HEMA-coated plates. Cells lysates were prepared and immunoprecipitated (IP) with anti-Cav-1 antibody. The resulting immune complexes were then analyzed for ubiquitin by Western blotting $(W B)$ at various times. Maximum ubiquitination of Cav-1 was observed at $\sim 3 \mathrm{~h}$ after cell detachment. Lysate input was determined by probing $\beta$-actin. CNTL, control. $B$, detached cells were similarly treated with the test agents, and Cav-1 S-nitrosylation was determined by immunoprecipitation using anti-Cav-1 antibody followed by Western blot analysis of the immunoprecipitated protein using anti-S-nitrosocysteine antibody. Densitometry was performed to determine the relative $S$-nitrosocysteine/ $\beta$-actin levels. $C, C a v-1 S$-nitrosylation was determined by fluorometry. Immunoprecipitates from above were incubated with $200 \mu \mathrm{M} \mathrm{HgCl}_{2}$ and $200 \mu \mathrm{M}$ diaminonaphthalene in phosphate-buffered saline. NO released from S-nitrosylated Cav-1 was quantified at 375/450 nm. Plots are the mean \pm S.D. $(n=3) .{ }^{*}, p<0.05$ versus non-treated control; $\#, p<$ 0.05 versus the indicated treatment controls.

Western blot analysis of the immunoprecipitated protein using $S$-nitrosocysteine antibody (Fig. 5B). Similar results were obtained when the immunoprecipitated protein was analyzed for $S$-nitrosylation by fluorometric measurements of the released NO product (Fig. 5C). Cav-1 $S$-nitrosylation by the NO donors was inhibited by DTT, a known inhibitor of $S$-nitrosylation $(37,38)$ (Fig. 5, $B$ and $C$ ), supporting the specificity of $S$-nitrosylation detection. To test whether $S$-nitrosylation of Cav-1 affects its stability, we analyzed the effect of NO donors and DTT on Cav-1 protein expression. Fig. $6 \mathrm{~A}$ shows that the NO donors SNP and DETA NONOate were able to stabilize the
A
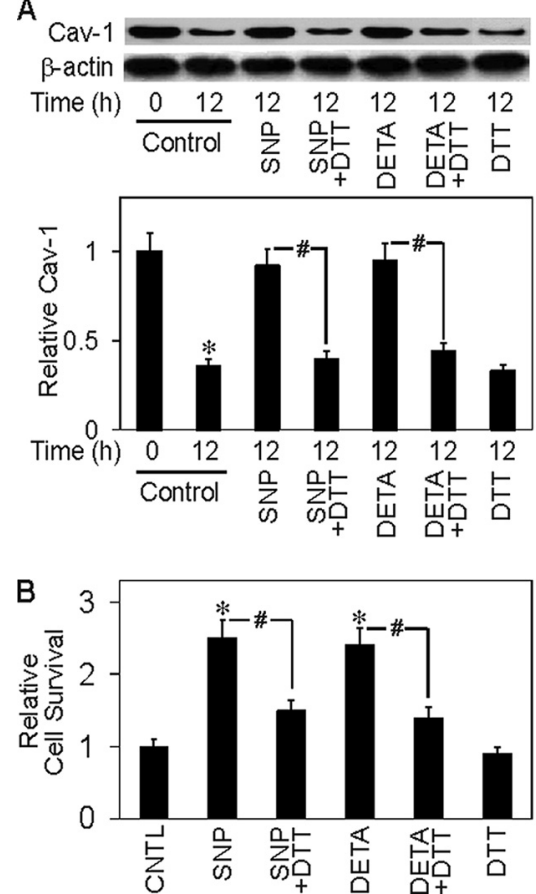

FIGURE 6. NO inhibits detachment-induced Cav-1 down-regulation and cell death. $A, \mathrm{H} 460$ cells were detached and either left untreated or treated with SNP $(50 \mu \mathrm{M})$ or DETA NONOate $(50 \mu \mathrm{M})$ in the presence or absence of DTT $(1 \mathrm{~mm})$ in HEMA-coated plates. $A$, cell lysates were prepared and analyzed for Cav-1 protein expression by Western blotting after $12 \mathrm{~h}$. Densitometry was performed to determine the relative levels of Cav-1 after reprobing the membranes with $\beta$-actin antibody. $B$, cells survival was determined by XTT assay after 12 h. C, morphology of cells treated with SNP $(50 \mu \mathrm{M})$ or DETA NONOate $(50 \mu \mathrm{M})$ for $12 \mathrm{~h}$. Data are the mean \pm S.D. $(n=3) .{ }^{*}, p<0.05$ versus nontreated control; \#, $p<0.05$ versus the indicated treatment controls.

protein after cell detachment and that the $S$-nitrosylation inhibitor DTT inhibited the stabilizing effect of NO donors. Together, these results indicate that NO regulates Cav-1 expression at least in part by inducing protein $S$-nitrosylation which interferes with its ubiquitination and subsequent degradation by the proteasome. This new finding provides a mechanistic insight into the regulation of Cav-1 by $\mathrm{NO}$, which could be important in the control of cancer cell anoikis and metastasis.

Nitric Oxide Induces Anoikis Resistance and Multilayer Formation-To provide supporting evidence for the role of $\mathrm{NO}$ in the regulation of cell anoikis through protein $S$-nitrosylation, $\mathrm{H} 460$ cells were detached and incubated with NO donors in the presence or absence of DTT. Cell viability was then determined after $12 \mathrm{~h}$ using XTT assay. Fig. $6 B$ shows that the NO donors SNP and DETA NONOate significantly increased cell viability after detachment and that DTT inhibited this effect of NO donors. In the earlier study we showed that cells overexpressing Cav-1 exhibited multilayer formation. Because NO up-regulates Cav-1 expression, we examined whether $\mathrm{NO}$ could induce a similar multilayer formation in $\mathrm{H} 460$ cells. Cells were grown in culture plates in the presence or absence of NO donors, and cell morphology was examined by microscopy after $24 \mathrm{~h}$. Fig. $6 B$ shows that the NO donors SNP and DETA NONOate were able to induce multilayer formation of $\mathrm{H} 460$ cells as compared with non-treated control. Because multilayer formation is a key characteristic of malignant tumor cells, this finding suggests 
that NO may regulate tumorigenesis by promoting malignant transformation through Cav-1 up-regulation.

\section{DISCUSSION}

Lung cancer is the leading cause of cancer mortality worldwide, and most death is associated with tumor metastasis. To metastasize, a malignant cell must detach from its primary tumor, invade the nearby circulatory or lymph system, and establish itself in a new site. Once in the bloodstream, most of the cells die by anoikis, which is an important mechanism that prevents metastasis. However, some cancer cells develop resistance to anoikis and consequently survive to establish new metastases. Several anoikis-regulatory proteins including Cav-1 have been investigated in recent years. However, the role of Cav-1 in metastatic cancer progression and the underlying mechanisms are unclear. Both pro- and anti-carcinogenic effect of Cav-1 have been described. Recombinant Cav-1 overexpression was shown to inhibit cell proliferation by inducing cell cycle arrest at $G_{0} / G_{1}$ phase (1). Genomic analysis of Cav$1^{-/-}$null mice and human breast cancer mutations (P132L) supported the role of Cav-1 as a negative regulator of cell transformation and tumorigenesis (39). Likewise, stable expression of Cav-1 in human breast cancer MCF-7 cells attenuated cell proliferation and inhibited anchorage-independent growth (17). In contrast to its suppressive role in cancer cell growth, an elevated expression of Cav-1 has been reported in several human tumors including prostate, colon, and breast $(23,24)$. Overexpression of Cav-1 also prevented detachment-induced apoptosis and p53 activation in cancer cells (18). Furthermore, in lung cancer cells Cav-1 overexpression promoted metastasis (19). Consistent with the pro-survival role of Cav-1, we found that Cav-1 positively regulated cell growth and inhibited detachment-induced apoptosis of lung cancer H460 cells (Fig. $3)$. The anti-apoptotic effect of Cav-1 was found to be associated with its ability to activate PI3K/Akt because inhibition of Akt activation by the PI3K inhibitor LY294002 abrogated this effect. Cav-1 has been shown to interact with and inhibit serine/ threonine protein phosphatases PP1 and PP2A, leading to sustained Akt activation and inhibition of apoptosis by thapsigargin in prostate cancer cells (16). Cav-1 has also been shown to interact with PI3K and regulate ceramide-induced cell death in fibroblasts (35). Likewise, Cav-1 overexpression in fibroblasts and HeLa cells induces Akt activation; however, such activation promotes cell death induced by arsenite (36). Thus, although there is a general agreement that Cav-1 can activate PI3K/Akt, its consequence on cell death can be different and is dependent on cellular context, cell type, and apoptotic stimuli used.

In this study we observed that Cav-1 is rapidly down-regulated after cell detachment and that overexpression of Cav-1 or stabilization of the protein by NO protected the cells from apoptosis (Figs. 3 and 6). NO induction and Cav-1 overexpression also promoted cell transformation facilitating multilayer formation (Figs. 3 and 6). The expression of Cav-1 is tightly regulated at various levels, including transcriptional and post-transcriptional (for review, see Ref. 40). Although the importance of transcriptional regulation of Cav-1 has been emphasized in numerous studies, post-translational modifications such as ubiquitination and phosphorylation have emerged as impor- tant regulators of protein stability and function (for reviews, see Refs. 29 and 41). In the present study we found that Cav-1 was rapidly ubiquitinated and degraded by the proteasome after cell detachment in concomitant with anoikis (Figs. 4 and 5). Cav-1 transcripts were relatively unchanged during this process (Fig. 4), indicating a non-transcriptional regulation of Cav-1 expression after cell detachment. These results support the ubiquitinproteasomal degradation as a primary mechanism of detachment-induced Cav-1 down-regulation. This finding adds Cav-1 to the growing list of cellular proteins that are subjected to regulation by the ubiquitin-proteasomal degradation pathway.

The results of this study also demonstrated that Cav-1 stability and function is regulated by NO. NO has been shown to regulate apoptosis under various physiologic and pathologic conditions $(28,42,43)$; however, its roles in anoikis and metastasis are unclear. Recent evidences suggest that depending on its expression level, NO can exert either promoting or inhibitory effects on tumor growth and metastasis. The promoting effects of NO are generally observed at relatively low but sustained levels of NO, whereas the inhibitory effects of NO are seen at high and acute concentrations that induce tumor cell death $(44-46)$. The results of this study are consistent with previous reports showing that low (micromolar) levels of NO inhibited cell death and increased cell migration (47-49). The promoting role of NO in tumorigenesis is also supported by the observations that both inducible and constitutive forms of NO synthase are elevated in several human tumors $(50,51)$ and that human and murine carcinomas expressing NO synthase are very aggressive when implanted into mice (52). Furthermore, there seems to be a direct correlation between the expression of NO synthase and the tumor grade, suggesting a causative role for NO in promoting metastasis (52).

We showed that NO negatively regulates anoikis of lung epithelial H460 cells as demonstrated by the ability of NO donors to suppress detachment-induced apoptosis and the reversal effect induced by $\mathrm{NO}$ inhibitors (Fig. 1). The NO donors also had a stabilizing effect on detachment-induced Cav-1 down-regulation, whereas the NO inhibitors showed an opposite effect (Figs. 4 and 6). These results support NO-mediated stabilization of Cav-1 as a key mechanism of anoikis regulation. The mechanism by which NO stabilizes Cav-1 was shown to involve inhibition of protein ubiquitination, as the NO donors inhibited the ubiquitination of Cav-1 by cell detachment (Fig. 5).

Recent evidence indicate that $S$-nitrosylation is an important mechanism by which NO modulates the function of cellular proteins (for reviews, see Refs. 29 and 53). S-Nitrosylation can either attenuate or accentuate protein functions $(34,54,55)$, and our previous studies have shown that it plays an important role in stability and function of apoptosis regulatory proteins such as c-FLIP and Bcl-2 (30-32). In the present study we found that Cav-1 was rapidly $S$-nitrosylated by NO after cell detachment, and inhibition of this $S$-nitrosylation by DTT blocked the effect of NO on Cav-1 ubiquitination (Fig. 5). The inhibition of $S$-nitrosylation by DTT also led to a decrease in Cav-1 protein expression and cell survival (Fig. 6), supporting the role of $S$-nitrosylation in Cav-1 stability and function. The mechanism by which $S$-nitrosylation promotes Cav-1 stability 
was shown to involve protein ubiquitination inhibition, although the precise mechanism of this inhibition remains to be investigated. It is possible that $S$-nitrosylation of Cav-1 may alter the protein conformation such that it could not be recognized by the enzyme ubiquitin ligases that serve to tag the protein for subsequent degradation by the proteasome.

In conclusion, we demonstrated that $\mathrm{Cav}-1$ plays an important role as a negative regulator of anoikis in human lung carcinoma $\mathrm{H} 460$ cells. We also demonstrated for the first time that Cav-1 is down-regulated during cell anoikis through a nontranscriptional mechanism involving ubiquitin-proteasomal degradation. NO regulates this process at least in part through protein $S$-nitrosylation, which prevents its ubiquitination and degradation by the proteasome. In demonstrating the effect of NO on Cav-1 stability and function, we documented a novel mechanism of anoikis regulation, which could be important in the control of cancer metastasis. Because NO and Cav-1 have been shown to be overexpressed in many human tumors, NOmediated regulation of Cav-1 could be a common mechanism of anoikis resistance and metastasis. This novel finding of the regulation of Cav-1 by $\mathrm{NO}$ may have important implications in cancer therapy.

\section{REFERENCES}

1. Galbiati, F., Volonté, D., Liu, J., Capozza, F., Frank, P. G., Zhu, L., Pestell, R. G., and Lisanti, M. P. (2001) Mol. Biol. Cell 12, 2229-2244

2. Glenney, J. R., Jr., and Zokas, L. (1989) J. Cell Biol. 108, 2401-2408

3. Rothberg, K. G., Heuser, J. E., Donzell, W. C., Ying, Y. S., Glenney, J. R., and Anderson, R. G. (1992) Cell 68, 673-682

4. Scherer, P. E., Okamoto, T., Chun, M., Nishimoto, I., Lodish, H. F., and Lisanti, M. P. (1996) Proc. Natl. Acad. Sci. U.S.A. 93, 131-135

5. Razani, B., Schlegel, A., Liu, J., and Lisanti, M. P. (2001) Biochem. Soc. Trans. 29, 494-499

6. Koleske, A. J., Baltimore, D., and Lisanti, M. P. (1995) Proc. Natl. Acad. Sci. U.S.A. 92, 1381-1385

7. Engelman, J. A., Chu, C., Lin, A., Jo, H., Ikezu, T., Okamoto, T., Kohtz, D. S., and Lisanti, M. P. (1998) FEBS Lett. 428, 205-211

8. Lee, S. W., Reimer, C. L., Oh, P., Campbell, D. B., and Schnitzer, J. E. (1998) Oncogene 16, 1391-1397

9. Hurlstone, A. F., Reid, G., Reeves, J. R., Fraser, J., Strathdee, G., Rahilly, M., Parkinson, E. K., and Black, D. M. (1999) Oncogene 18, 1881-1890

10. Racine, C., Bélanger, M., Hirabayashi, H., Boucher, M., Chakir, J., and Couet, J. (1999) Biochem. Biophys. Res. Commun. 255, 580-586

11. Galbiati, F., Volonte, D., Engelman, J. A., Watanabe, G., Burk, R., Pestell, R. G., and Lisanti, M. P. (1998) EMBO J. 17, 6633-6648

12. Campbell, L., Hollins, A. J., Al-Eid, A., Newman, G. R., von Ruhland, C., and Gumbleton, M. (1999) Biochem. Biophys. Res. Commun. 262, 744-751

13. Mikol, D. D., Hong, H. L., Cheng, H. L., and Feldman, E. L. (1999) Glia 27, $39-52$

14. Timme, T. L., Goltsov, A., Tahir, S., Li, L., Wang, J., Ren, C., Johnston, R. N., and Thompson, T. C. (2000) Oncogene 19, 3256-3265

15. Li, L., Yang, G., Ebara, S., Satoh, T., Nasu, Y., Timme, T. L., Ren, C., Wang, J., Tahir, S. A., and Thompson, T. C. (2001) Cancer Res. 61, 4386-4392

16. Li, L., Ren, C. H., Tahir, S. A., Ren, C., and Thompson, T. C. (2003) Mol. Cell. Biol. 23, 9389-9404.

17. Fiucci, G., Ravid, D., Reich, R., and Liscovitch, M. (2002) Oncogene 21, 2365-2375

18. Ravid, D., Maor, S., Werner, H., and Liscovitch, M. (2005) Oncogene 24, $1338-1347$

19. Ho, C. C., Huang, P. H., Huang, H. Y., Chen, Y. H., Yang, P. C., and Hsu, S. M. (2002) Am. J. Pathol. 161, 1647-1656

20. Liu, C. Y., Wang, C. H., Chen, T. C., Lin, H. C., Yu, C. T., and Kuo, H. P. (1998) Br. J. Cancer 78, 534-541

21. Arias-Díaz, J., Vara, E., Torres-Melero, J., García, C., Baki, W., Ramírez-
Armengol, J. A., and Balibrea, J. L. (1994) Cancer 74, 1546-1551

22. Fujimoto, H., Ando, Y., Yamashita, T., Terazaki, H., Tanaka, Y., Sasaki, J., Matsumoto, M., Suga, M., and Ando, M. (1997) Jpn. J. Cancer Res. 88, $1190-1198$

23. Yang, G., Truong, L. D., Timme, T. L., Ren, C., Wheeler, T. M., Park, S. H., Nasu, Y., Bangma, C. H., Kattan, M. W., Scardino, P. T., and Thompson, T. C. (1998) Clin. Cancer Res. 4, 1873-1880

24. Thompson, T. C. (1998) Cancer Metastasis Rev. 17, 439-442

25. Thomsen, L. L., Miles, D. W., Happerfield, L., Bobrow, L. G., Knowles, R. G., and Moncada, S. (1995) Br. J. Cancer 72, 41-44.

26. Ambs, S., Merriam, W. G., Bennett, W. P., Felley-Bosco, E., Ogunfusika, M. O., Oser, S. M., Klein, S., Shields, P. G., Billiar, T. R., and Harris, C. C. (1998) Cancer Res. 58, 334-341

27. Cobbs, C. S., Brenman, J. E., Aldape, K. D., Bredt, D. S., and Israel, M. A. (1995) Cancer Res. 55, 727-730

28. Heigold, S., Sers, C., Bechtel, W., Ivanovas, B., Schäfer, R., and Bauer, G. (2002) Carcinogenesis 23, 929-941

29. Iyer, A. K., Azad, N., Wang, L., and Rojanasakul, Y. (2008) Nitric Oxide 19, $146-151$

30. Chanvorachote, P., Nimmannit, U., Wang, L., Stehlik, C., Lu, B., Azad, N., and Rojanasakul, Y. (2005) J. Biol. Chem. 280, 42044-42050

31. Chanvorachote, P., Nimmannit, U., Stehlik, C., Wang, L., Jiang, B. H., Ongpipatanakul, B., and Rojanasakul, Y. (2006) Cancer Res. 66, 6353-6360

32. Azad, N., Vallyathan, V., Wang, L., Tantishaiyakul, V., Stehlik, C., Leonard, S. S., and Rojanasakul, Y. (2006) J. Biol. Chem. 281, 34124-34134.

33. Livak, K. J., and Schmittgen, T. D. (2001) Methods 25, 402- 408

34. Xu, L., Han, C., Lim, K., and Wu, T. (2008) J. Biol. Chem. 283, 3077-3087

35. Zundel, W., Swiersz, L. M., and Giaccia, A. (2000) Mol. Cell. Biol. 20, $1507-1514$

36. Shack, S., Wang, X. T., Kokkonen, G. C., Gorospe, M., Longo, D. L., and Holbrook, N. J. (2003) Mol. Cell. Biol. 23, 2407-2414

37. Ryu, S. D., Yi, H. G., Cha, Y. N., Kang, J. H., Kang, J. S., Jeon, Y. C., Park, H. K., Yu, T. M., Lee, J. N., and Park, C. S. (2004) Life Sci. 75, 2559-2572

38. Moon, K. H., Kim, B. J., and Song, B. J. (2005) FEBS Lett. 579, 6115-6120

39. Williams, T. M., Lee, H., Cheung, M. W., Cohen, A. W., Razani, B., Iyengar, P., Scherer, P. E., Pestell, R. G., and Lisanti, M. P. (2004) J. Biol. Chem. 279, 24745-24756

40. Williams, T. M., and Lisanti, M. P. (2005) Am. J. Physiol. Cell Physiol. 288, C494-C506

41. Hershko, A., and Ciechanover, A. (1998) Annu. Rev. Biochem. 67, $425-479$

42. Borutaite, V., and Brown, G. C. (2003) Free Radic. Biol. Med. 35, $1457-1468$

43. Souici, A. C., Mirkovitch, J., Hausel, P., Keefer, L. K., and Felley-Bosco, E. (2000) Carcinogenesis 21, 281-287

44. Mocellin, S., Bronte, V., and Nitti, D. (2007) Med. Res. Rev. 27, 317-352

45. Lala, P. K., and Chakraborty, C. (2001) Lancet Oncol. 2, 149-156

46. Monteiro, H. P., Silva, E. F., and Stern, A. (2004) Nitric Oxide 10, 1-10

47. Antonova, G. N., Snead, C. M., Antonov, A. S., Dimitropoulou, C., Venema, R. C., and Catravas, J. D. (2007) Am. J. Physiol. Heart Circ. Physiol. 292, H893-H903

48. Choi, B. M., Pae, H. O., and Chung, H. T. (2003) Free Radic. Biol. Med. 34, $1136-1145$

49. Dimmeler, S., and Zeiher, A. M. (1999) Cell Death Differ. 6, 964-968

50. Park, S. W., Lee, S. G., Song, S. H., Heo, D. S., Park, B. J., Lee, D. W., Kim, K. H., and Sung, M. W. (2003) Int. J. Cancer 107, 729-738

51. Ambs, S., Bennett, W. P., Merriam, W. G., Ogunfusika, M. O., Oser, S. M., Khan, M. A., Jones, R. T., and Harris, C. C. (1998) Br. J. Cancer 78, 233-239

52. Jenkins, D. C., Charles, I. G., Thomsen, L. L., Moss, D. W., Holmes, L. S., Baylis, S. A., Rhodes, P., Westmore, K., Emson, P. C., and Moncada, S. (1995) Proc. Natl. Acad. Sci. U.S.A. 92, 4392-4396

53. Hess, D. T., Matsumoto, A., Kim, S. O., Marshall, H. E., and Stamler, J. S. (2005) Nat. Rev. Mol. Cell Biol. 6, 150-166

54. Li, J., Billiar, T. R., Talanian, R. V., and Kim, Y. M. (1997) Biochem. Biophys. Res. Commun. 240, 419-424

55. Kim, Y. M., Talanian, R. V., and Billiar, T. R. (1997) J. Biol. Chem. 272, $31138-31148$ 


\title{
A Shope Fibroma virus PYRIN-only protein modulates the host immune response
}

\author{
Andrea Dorfleutner - Siera J. Talbott · Nicole B. Bryan • \\ Kristin N. Funya - Stephanie L. Rellick · John C. Reed • \\ Xianglin Shi · Yon Rojanasakul · Daniel C. Flynn • \\ Christian Stehlik
}

Received: 7 August 2006/Accepted: 29 November 2006/Published online: 4 August 2007

(C) Springer Science+Business Media, LLC 2007

\begin{abstract}
PYRIN domain (PYD) proteins have recently emerged as important signaling molecules involved in the development of innate immunity to intracellular pathogens through activation of inflammatory mediator pathways. ASC is the central adaptor protein, which links pathogen recognition by PYD-containing pathogen recognition receptors to the activation of downstream effectors, including activation of Caspase- 1 and NF- $\kappa \mathrm{B}$. The cellular PYD-only protein 1 (cPOP1) can block the recruitment of ASC to activated PAN receptors and thereby functions as an endogenous inhibitor of the PYD-mediated signal transduction pathway. Here we describe the identification and characterization of a Shope Fibroma homolog to
\end{abstract}

Andrea Dorfleutner and Siera J. Talbott contributed equally to this work.

A. Dorfleutner $\cdot$ S. J. Talbott $\cdot$ N. B. Bryan

K. N. Funya - S. L. Rellick - D. C. Flynn - C. Stehlik $(\bowtie)$

Mary Babb Randolph Cancer Center and Department

of Microbiology, Immunology \& Cell Biology,

West Virginia University School of Medicine, 1826 MBRCC,

1 Medical Center Drive, Morgantown, WV 26506-9300, USA

e-mail: cstehlik@hsc.wvu.edu

J. C. Reed

Burnham Institute for Medical Research, 10901 North

Torrey Pines Road, La Jolla, CA 92037, USA

X. Shi

Pathology and Physiology Research Branch, Health Effects

Laboratory Division, National Institute for Occupational

Safety and Health, Morgantown, WV 26505, USA

Y. Rojanasakul

Department of Pharmaceutical Sciences, School of Pharmacy,

West Virginia University, 1 Medical Center Drive,

Morgantown, WV 26506-9500, USA
cPOP1. Like cPOP1, a Shope Fibroma virus-encoded POP (vPOP), co-localizes and directly associates with ASC and inhibits PYD-mediated signal transduction. Poxviruses are known to encode immune evasive proteins to promote host cell infection and suppression of the host immune response. Poxvirus-encoded vPOPs represent a novel class of immune evasive proteins and impair the host response by blocking Cryopyrin and ASC inflammasome-mediated activation of pro-Caspase- 1 and subsequent processing of pro-interleukin (IL)-1 $\beta$, and expression of vPOPs causes activation of NF- $\kappa \mathrm{B}$.

Keywords Caspase- $1 \cdot$ IL-1 $\beta$ - Inflammasome · NF- $\kappa$ B $\cdot$ PYRIN domain $\cdot$ Poxvirus

\section{Introduction}

PYRIN domain (PYD) proteins are important signaling molecules involved in host defense to pathogens through activation of inflammatory mediator pathways. Humans have at least 23 proteins containing a PYD. [1-4]. Pathogens are recognized by the leucine-rich region (LRR) of PYD-containing pathogen recognition receptors, known as PYD-NLR, PAN, PYPAF, NALP, Caterpiller, and Nod proteins. Activation of these proteins is hypothesized to be achieved through displacement of the intramolecular interaction of the LRR with the NACHT domain. Activated PYD-NLR-family proteins recruit and oligomerize the PYD-containing adapter protein ASC (TMS1) [5, 6] into cytoplasmic aggregates ("specks"), referred to as the inflammasome, where activation of downstream effectors occurs [7]. Caspase-1-mediated processing of pro-IL-1 $\beta$ and activation of the transcription factor NF- $\kappa \mathrm{B}$ have been described as effectors of PYD-mediated signal transduction 
and enforced oligomerization of ASC is sufficient for activation of these downstream effectors.

Hereditary mutations in PYD-NLR-family proteins can cause deregulated recruitment of ASC, which leads to autoinflammatory disorders, such as periodic fever syndromes. Specifically, mutations in the PYD-containing protein Pyrin (Marenostrin) account for familial Mediterranean fever, whereas mutations in the PYD-NLR-family member Cryopyrin (CIAS1, PYPAF1, NALP3) have been linked to familial cold auto-inflammatory Syndrome, Muckle-Wells syndrome, and Chronic infantile neurological cutaneous and articular syndrome [8].

The PYD belongs to the family of death domain fold (DDF) domains, which also includes the caspase recruitment domain (CARD), the death domain (DD), and the death effector domain (DED). CARD and DED interactions are also regulated by CARD-only proteins (COPs) and DED-only proteins, respectively. These small proteins are just composed of a single CARD or DED and include the CARD-only proteins COP (Pseudo-ICE), Iceberg and INCA [9-12], or the DED-only proteins PED (PEA-15), FLIP-s, and viral (v) FLIP [13, 14]. In general DDF-only proteins can function as dominant-negative inhibitors for particular signaling pathways by competing for critical binding partners, thereby interrupting signal transmission to downstream effector proteins.

The recruitment of ASC to PYD-NLR-family proteins is regulated by a PYRIN-only protein 1 (POP1), which interferes with the PYD-PYD interaction between ASC and PYD-NLRs, thereby impairing host defense by blocking downstream effectors [15]. Here we report the identification and characterization of a Shope Fibroma Virus (SFV) POP homolog, which impairs the host response by blocking PYD-mediated activation of pro-Caspase-1 and subsequent processing of pro-interleukin (IL)- $1 \beta$. During the preparation of this manuscript, Johnston et al. characterized a Myxoma virus-encoded POP, which also functions as an inhibitor of IL-1 $\beta$ secretion [16]. We continued this study and show that the Shope Fibroma virus-encoded POP functions similar to the Myxoma virus-encoded POP, and that both proteins affect also NF- $\kappa \mathrm{B}$ activation.

\section{Materials and methods}

Plasmids and strains

The rabbit Myxoma virus (MV; strain Lausanne) and the rabbit Shope Fibroma virus (SFV; strain Original A) were obtained from the American Type Culture Collection. The complete open reading frame of M013L was cloned directly from the genome of MV by high fidelity PCR (PFU Ultra, Stratagene) using the primer pair
5'-CGGAATTCAAAATGGAGCACCGACCGTCATTA-3', 5'-GCGCCTCGAGATAAAATAACAATTTGCGACATA CACC- $3^{\prime}$. The complete open reading frame of S013L was cloned directly from the genome of SFV using the primer pair 5'-CGGAATTCTACTTTAAAATGAAGGAAAAT GACAT-3', 5'-GCGCCTCGAGTTGATAAAACAAACT TTCACGATA-3'. Both PCR products were digested with EcoR1 and Xho1 restriction enzymes and cloned into pcDNA3 (Invitrogen) expression plasmids modified to contain an $\mathrm{NH}_{2}$-terminal myc or Flag epitope tag. The authenticity of S013L and M013L was confirmed by DNA sequence analysis. Expression constructs for ASC, ASCPYD, ASC-CARD, cPOP1, Cryopyrin (R260W), proIL-1 $\beta$, and pro-Caspase-1 were described previously $[15,17,18]$.

\section{Cell culture and transfection}

HEK293N, HEK293T, and rabbit kidney (RK13) cells were obtained from the American Type Culture Collection and cultured in Dulbecco's modified Eagle's medium supplemented with $4 \mathrm{mM}$ L-glutamine, $1.5 \mathrm{~g} / 1$ sodium bicarbonate, $0.1 \mathrm{mM}$ non-essential amino acids, $1.0 \mathrm{mM}$ sodium pyruvate, and $10 \%$ fetal bovine serum (HEK293) and Minimum essential medium (Eagle) with $2 \mathrm{mM}$ L-glutamine and Earle's BSS adjusted to contain $1.5 \mathrm{~g} / \mathrm{l}$ sodium bicarbonate, $0.1 \mathrm{mM}$ non-essential amino acids, $1.0 \mathrm{mM}$ sodium pyruvate, and $10 \%$ fetal bovine serum (RK13). HEK293 cells were transfected using Polyfect (Qiagen) or the Calcium precipitation method.

\section{Viral infection}

Infection of subconfluent layers of RK13 cells with the rabbit Fibroma virus (strain Original A) was carried out at a multiplicity of infection (MOI) of 10 in OPTI minimum essential medium (Invitrogen) supplemented with $2 \%$ fetal calf serum for $1 \mathrm{~h}$. The inoculum was removed, the cells were washed with serum-free medium and further grown for the indicated times.

Reverse transcriptase-polymerase chain (RT-PCR) reaction

RK13 cells were infected with the rabbit SFV (strain Original A) at a MOI of 10, as described above. Sixteen hours $(16 \mathrm{~h})$ post-infection, RNA was isolated using the Trizol reagent (Invitrogen) according to the manufacturer's instructions. Five micrograms $(5 \mu \mathrm{g})$ total RNA was subjected to DNase I (Invitrogen) treatment according to the manufacturer's instructions and reverse transcribed using the Superscript II enzyme (Invitrogen) into first strand cDNA, followed by PCR amplification with GoTaq poly- 
merase (Promega) using the S013L-specific primer pair 5'-CGGAATTCTACTTTAAAATGAAGGAAAATGAC AT-3', 5'-GCGCCTCGAGTTGATAAAACAAACTTTCA CGATA-3' (324 bp) and the $\beta$ ACTIN-specific primer pair 5'-GACGATGATATTGCCGCACT-3', and 5'-GATACCA CGCTTGCTCTGAG-3' (533 bp). A control, in which the RT was omitted during the RT step, was also performed. PCR-products were excised from agarose gels and sequence verified.

\section{Co-immunoprecipitation assay}

Immunoprecipitation assays were performed following transient expression of HA-tagged ASC and either myctagged M013L or Myc-tagged gp013L into HEK293T cells. Thirty-six hours ( $36 \mathrm{~h})$ post-transfection, cells were lysed in isotonic lysis buffer $(150 \mathrm{mM} \mathrm{NaCl}, 20 \mathrm{mM}$ Tris$\mathrm{HCl}, \mathrm{pH} 7.4,10 \%$ glycerol, $0.2 \%$ NP-40), supplemented with protease and phosphatase inhibitors. Clarified lysates were subjected to immunoprecipitation using agaroseconjugated anti-HA antibodies (Santa Cruz Biotechnology) for $4 \mathrm{~h}$ at $4^{\circ} \mathrm{C}$. Following extensive washing in lysis buffer, bound immune complexes were separated by SDS/PAGE and analyzed by immunoblotting using directly HRP-conjugated anti-myc antibodies (Santa Cruz Biotechnology) in conjunction with an ECL detection system (AmershamPharmacia). Where indicated, cell lysates (10\% volume) were included along side immune complexes. Alternatively, lysates were directly analyzed by immunoblotting after normalization for total protein content.

In vitro protein interaction assay

The PYD of ASC was expressed as a GST-fusion protein in E. coli BL21 cells (Stratagene) and affinity-purified using GSH-Sepharose (Amersham-Pharmacia). GST-ASC-PYD or GST control (50 ng) immobilized on $10 \mu \mathrm{l} \mathrm{GSH}$ Sepharose were blocked in $142.4 \mathrm{mM} \mathrm{KCl}, 5 \mathrm{mM} \mathrm{MgCl}$, $10 \mathrm{mM}$ HEPES $\mathrm{pH}$ 7.4, $0.5 \mathrm{mM}$ EGTA, $1 \mathrm{mM}$ EDTA, $0.2 \%$ Nonidet P-40, $1 \mathrm{mM}$ DTT, supplemented with protease inhibitors and $1 \mathrm{mg} / \mathrm{ml} \mathrm{BSA}$ for $30 \mathrm{~min}$ at room temperature. Beads were washed twice and incubated with in vitro translated and biotinylated (Promega) vPOP proteins overnight at $4^{\circ} \mathrm{C}$ as above, but the buffer was supplemented with protease inhibitors and $0.5 \mathrm{mg} / \mathrm{ml}$ BSA. Bound proteins were washed extensively, separated by SDS/PAGE, immunoblotted with Streptavidin-HRP and detected with ECL (Amersham-Pharmacia).

Confocal microscopy observations

RK13 cells were seeded onto Collagen type I-coated cover slips in 6-well dishes and transiently transfected the following day with expression plasmids as indicated using Lipofectamine plus (Invitrogen). Where indicated, cells were infected the following day with SFV (MOI = 10). At 48 and $72 \mathrm{~h}$ post-transfection ( 24 and $48 \mathrm{~h}$ post-infection), cells were fixed in $3.7 \%$ formaldehyde, permeabilized with $0.5 \%$ Saponin (Sigma), blocked with $0.5 \%$ Saponin, $1.5 \%$ BSA, and $1.5 \%$ normal goat serum (Zymed), and immunostained with rabbit polyclonal anti-myc antibodies (1:500, Santa Cruz Biotech), mouse monoclonal anti-Flag M2 antibodies (1:3,500, Sigma) and secondary Alexa Fluor 488 and 546 conjugated antibodies (1:200, Molecular Probes) in PBS, supplemented with $0.5 \%$ Saponin, $1.5 \%$ BSA, and $1.5 \%$ normal goat serum (Zymed). The nucleus is visualized by incubation with ToPro-3 (Molecular Probes) and the actin cytoskeleton with Alexa Fluor-conjugated phalloidin. After three washes with PBS, supplemented with $0.5 \%$ Saponin, samples were mounted with Vectashield (Vectorlabs) and images were acquired by confocal laser-scanning microscopy (Zeiss LSM510).

\section{Luciferase reporter gene assay}

About $5 \times 10^{4}$ HEK293N cells cultured in 5\% serum in 96well plates were transfected in triplicates with the indicated expression plasmids using Polyfect transfection reagent (Qiagen) with a total of $500 \mathrm{ng}$ plasmid DNA (normalized for total DNA), including $75 \mathrm{ng}$ of $\mathrm{pNF}-\kappa \mathrm{B}-\mathrm{LUC}$ (Stratagene) and $3 \mathrm{ng}$ of a Renilla luciferase gene driven by a constitutive TK promoter (pRL-TK; Promega). At $36 \mathrm{~h}$ post-transfection, cells were treated where indicated with $20 \mathrm{ng} / \mathrm{ml} \mathrm{TNF} \alpha$ (Biomol) for $8 \mathrm{~h}$ and directly analyzed using the Dual Glow Luciferase kit (Promega) in a Genios multimode plate reader (Tecan).

\section{IL-1 $\beta$ secretion assay}

HEK293N cells were transiently transfected with the indicated expression plasmids in 24-well plates using the Polyfect transfection reagent (Qiagen). Twenty-four hours (24 h) post-transfection, the culture medium was replenished with $0.5 \mathrm{ml}$ of fresh culture medium. Thirty-six hours (36 h) post-transfection, IL- $1 \beta$ secreted into the culture supernatants was measured by ELISA using a commercial kit (BD Pharmingen), normalizing data for cell number, as determined by crystal violet assay, and precise culture volume, and performing assays in triplicates.

\section{Results}

Poxviruses encode PYD-only proteins

Viruses are known to interfere with host immune and apoptosis pathways. Since the PYD emerged recently as a 
key domain in innate immunity, we performed database mining to identify potential virus-encoded PYD-containing proteins. Using this approach, we identified open reading frames in several poxviruses that encode potential viral PYD-only proteins (vPOPs) (Fig. 1A). Clustal W alignment of the deduced amino acid sequences of the rabbit Myxoma virus (MV) POP (M013L) [20], the Shope Fibroma virus (SFV) POP (gene: S013L, protein gp013L) [21], the Swinepox virus (SPV) POP (SPV14L) [22], the Yaba-like disease virus (YLDV) POP (18L) [23], and the Mule deer poxvirus (DpV) POP (DPV83gp024) [24] show significant homologies to cPOP1 and the PYD of ASC (Fig. 1B). The M013L and gp013L proteins are only 59.5\% identical $(65 \%$ conserved) to each other. To verify that S013L is also transcribed in cells infected with poxviruses, we performed RT-PCR analysis of S013L, due to the lack of specific antibodies. Total RNA was isolated from RK13 cells and RK13 cells infected with the SFV for $16 \mathrm{~h}$, and transcribed into first strand cDNAs. Subsequent PCR analysis using S013L-specific primers demonstrated the presence of vPOP-specific transcripts only in SFV-infected cells, which show the typical cytopathic effects (Fig. 1C, lane 3). Primer specificity for vPOP versus cPOP is shown by the absence of PCR product in non-infected cells (Fig. 1C, lane 2). Viral genomic DNA-contamination was excluded by omitting cDNA from the reverse transcription in the PCR reaction, which did not amplify a PCR product (Fig. 1C, lane 4).

For further studies we amplified the ORF of S013L and M013L by PCR directly from virus particles, cloned it into mammalian expression vectors in frame with a myc-epitope tag and sequence verified these vPOPs. Transient transfection of S013L and M013L in HEK293 cells resulted in expression of vPOPs of the predicted molecular weight (Fig. 1D). Myc-tagged cPOP1 was used as a control.
A

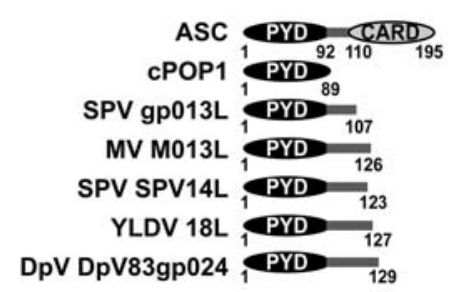

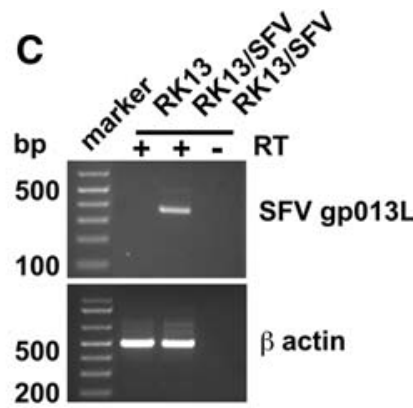

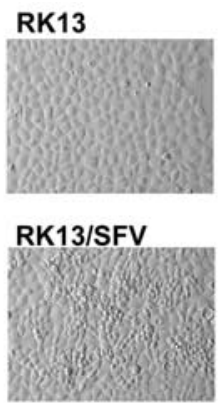

D

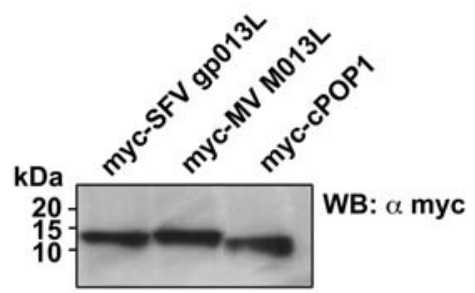

B
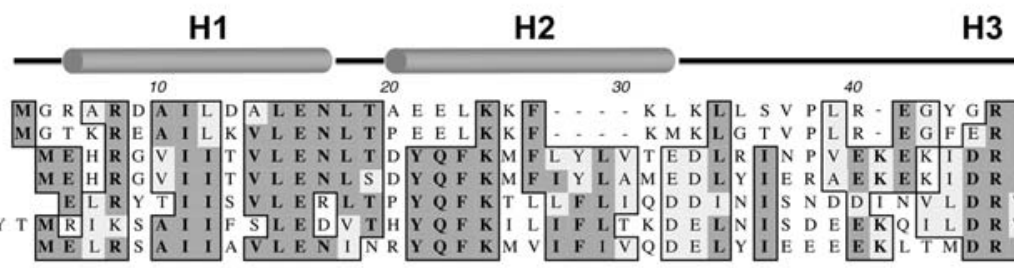

H3

40

20 $\mathrm{H} 4$

ASC-PYD

cPOP1

SFV gp013L

MV M013L

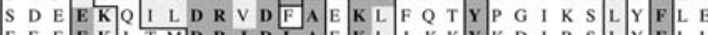
DpV 83gpo

H5

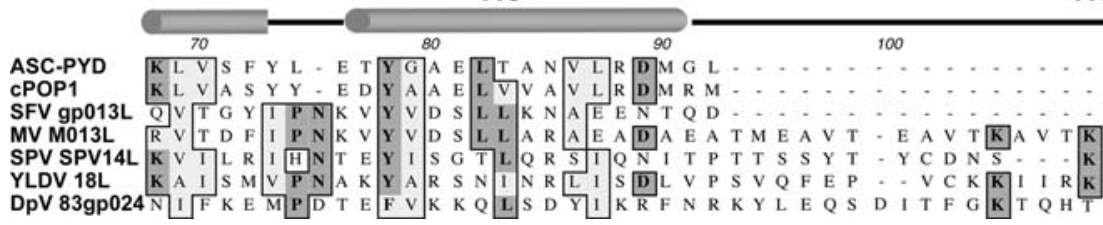

H6

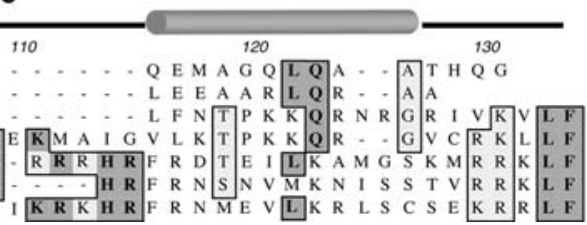

Fig. 1 The Shope Fibroma virus encodes a PYD-only protein. Comparison of viral and cellular PYD-only proteins. (A) Schematic representation of Myxoma virus (MV) POP (M013L), the Shope Fibroma virus (SFV) POP (gp013L), the Swinepox virus (SPV) POP (SPV14L), the Yaba-like disease virus (YLDV) POP (18L), the Mule deer poxvirus (DpV) POP (DPV83gp024), cPOP1, and ASC. (B) Clustal W alignment of M013L, gp013L, SPV14L, 18L, DPV83gp024, cPOP1 and the PYD of ASC. Black and gray boxes indicate identical and similar (conserved) amino-acid residues, respectively. The $\alpha$-helices, as determined for the PYD of ASC are marked on top [19]. (C) The presence of S013L-specific transcripts was determined in RK13 cells before and $16 \mathrm{~h}$ post-infection with the rabbit Fibroma virus $(\mathrm{SFV})(\mathrm{MOI}=10)$ by RT-PCR. RT-PCR was performed with S013L-specific primers and primers specific for $\beta$ actin. RT: reverse transcriptase. A representative phase contrast image of uninfected RK13 cells (top) and RK13 cells infected with SFV (bottom) is shown. (D) Myc-tagged expression constructs for cPOP1, SFV gp013L, and MV M013L were transiently transfected into HEK293T cells. Thirty-six hours $(36 \mathrm{~h})$ post-transfection, normalized cell lysates were separated by SDS/PAGE and analyzed by immunoblot for expression of myc-tagged proteins 
gp013L co-localizes with the central PYD-containing adaptor protein ASC

The central adaptor protein ASC binds to activated PYD-NLR-family proteins and mediates activation of downstream effectors by PYD-PYD interactions. ASC frequently localizes to characteristic aggregates, referred to as specks [6], where ASC interacting proteins such as cPOP1 are frequently recruited [15]. To investigate whether SFV gp013L co-localizes with ASC similar to cPOP1 and MV M013L, we performed immunofluorescence analysis of cellular and viral POPs in the absence or presence of ASC. Myc-tagged SFV gp013L (Fig. 2A-a), or myc-tagged MV M013L (Fig. 2B-a) alone localized to diffusively throughout the cytoplasm and the nucleus at $48 \mathrm{~h}$ post-transfection. At $72 \mathrm{~h}$ post-transfection, SFV gp013L (Fig. 2A-b) and MV M013L (Fig. 2B-b) localized to punctuate, vesicular structures in the cytoplasm. To investigate whether virus infection impacts the localization of vPOPs, we transiently transfected SFV gp013L into RK13 cells, followed by infection with SFV. Twenty-four hours (24 h) post-infection SFV gp013L started to aggregate in the cytoplasm of infected cells (Fig. 2A-c), with stronger aggregation visible at $48 \mathrm{~h}$ post-infection (Fig. 2 A-d).

Also cPOP1 showed cytoplasmic and nuclear localization, and co-expression of cPOP1 and ASC leads to recruitment of cPOP1 into ASC-formed specks [15]. Therefore, localization of vPOPs to these structures was investigated. Co-expression of Flag-tagged ASC resulted in co-localization of SFV gp013L (Fig. 2A-e) and MV M013L (Fig. 2B-c) proteins with ASC in the characteristic specks. Infection of RK13 cells with SFV did not impact the localization of ASC and SFV gp013L, likely because these proteins already localize to specks (Fig. 2A-f).

gp013L interacts with the central PYD-containing adaptor protein ASC

cPOP1 co-localizes with ASC into ASC-formed specks and has been identified as an ASC-binding protein. Since SFV gp013L, and MV M013L co-localized with ASC, their capability to interact with each other was investigated. Myc-tagged SFV gp013L or MV M013L were co-expressed in HEK293T cells with HA-tagged ASC by transient co-transfection. Co-immunoprecipitation experiments of cleared protein lysates with anti-HA sepharose demonstrated the presence of myc-tagged SFV gp013L or MV M013L in these immune complexes, further indicating that SFV gp013L and MV M013L form complexes with ASC in vivo, reminiscent to cPOP1 (Fig. 3A).

PYD-containing proteins usually associate by PYDPYD interaction. POPs only contain the PYD, but ASC consists of a PYD and a CARD [5, 6]. To map the interaction domain of ASC and SFV gp013L or MV M013L, we performed in vitro GST-pull down assays. Recombinant proteins of the PYD or the CARD of ASC were expressed as GST fusion proteins in E. coli BL21 and affinity purified, whereas SFV gp013L and MV M013L were generated by in vitro translation. These in vitro interaction studies further confirmed that SFV gp013L and MV M013L interact with the PYD of ASC, while no interaction was observed with the CARD of ASC or the GST control (Fig. 3B). These results demonstrated that SFV gp013L and MV M013L directly interact with ASC by PYD-PYD interaction.

gp013L interferes with PYD-mediated activation of Caspase-1

The best-characterized effector protein downstream of the PYD signal transduction pathway is Caspase-1. Interaction of cPOP1 with ASC impairs PYD-mediated activation of downstream effectors by preventing association with upstream PYD-NLR-family proteins [15]. Since vPOPs share significant homology with cPOP1, we investigated the effect of SFV gp013L or MV M013L interaction with ASC on PYD-mediated activation of Caspase-1. The PYD signal transduction pathway was reconstituted in HEK293N cells, which are deficient in endogenous components of this pathway, including ASC. Co-expression of a constitutively active Cryopyrin R260W (a PYD-NLR-family member), ASC, pro-Caspase-1, and pro-IL- $1 \beta$ leads to activation of the PYD-mediated signal transduction pathway, and can be measured by secretion of bioactive IL- $1 \beta$. Co-expression of the disease-associated Cryopyrin mutant R260W interacts with ASC even in the absence of a ligand, promotes ASC oligomerization, activation of pro-Caspase-1, and subsequently IL- $1 \beta$ secretion [25]. Co-expression of either SFV gp013L (Fig. 4A) or MV M013L (Fig. 4B) in this system impaired ASC-mediated activation of Caspase-1 in a dosedependent manner and reduced activity of Caspase- 1 to baseline, as measured by secretion of bioactive IL- $1 \beta$, which is processed from pro-IL- $1 \beta$ into bioactive IL- $1 \beta$ by Caspase-1. Expression of all proteins, except IL-1 $\beta$, which we measured by ELISA, was verified by immunoblot (Fig. 4C).

gp013L promotes activation of the transcription factor $\mathrm{NF}-\kappa \mathrm{B}$

Recruitment of ASC to activated PYD-NLR-family members, such as Cryopyrin, has also been implicated in the activation of the transcription factor $\mathrm{NF}-\kappa \mathrm{B}$, though this finding is not yet supported by animal studies [17, 26]. cPOP1 can interfere with the interaction of ASC and 


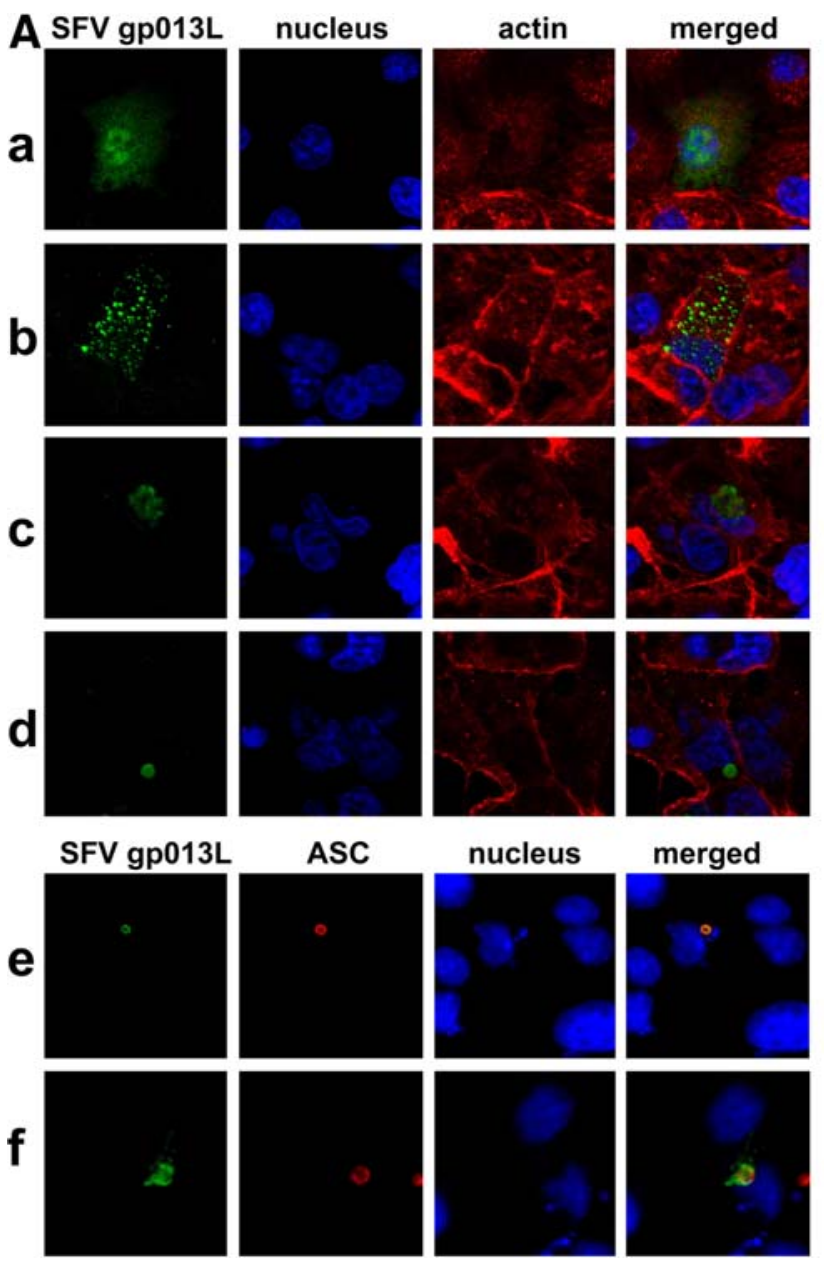

Fig. 2 SFV gp013L co-localizes with ASC. Localization of epitopetagged proteins was analyzed following transient transfection into RK13 cells. (A) Localization of SFV gp013L. Myc-tagged SFV gp013L was immunostained with a rabbit polyclonal anti-myc antibody and visualized with an Alexa Fluor 488-conjugated antirabbit antibody $48 \mathrm{~h}$ post-transfection (panel a) and $72 \mathrm{~h}$ posttransfection (panel b). Actin was visualized with Alexa Fluor 546conjugated phalloidin and the nucleus was stained with ToPro-3. Shown is from left to right: Myc-tagged SFV gp013L (green), nucleus (blue), actin (red), and a merged image. Twenty-four hours $(24 \mathrm{~h})$ post-transfection cells were also infected with SFV $(\mathrm{MOI}=10)$ and cells were immunostained as described above at 24 (panel c) and $48 \mathrm{~h}$ (panel d) post-infection (48 and $72 \mathrm{~h}$ post-transfection). Myc-tagged SFV gp013L and Flag-tagged ASC were immunostained $72 \mathrm{~h}$ posttransfection with rabbit polyclonal anti-myc and mouse monoclonal anti-Flag antibodies and visualized with Alexa Fluor 488 and 546conjugated anti-rabbit and mouse antibodies, respectively. The nucleus was stained with ToPro-3. Shown is from left to right: Myc-tagged SFV gp013L (green), Flag-tagged ASC (red), nucleus

Cryopyrin, thereby impairing oligomerization of ASC and subsequently activation of NF- $\kappa \mathrm{B}$ [15]. Therefore, the effect of SFV gp013L and MV M013L on NF- $\kappa$ B activation was investigated. Expression of SFV gp013L and MV M013L alone potently activated NF- $\kappa \mathrm{B}$, in contrast to cPOP1 (Fig. 5A). In contrast to the inhibitory effect of cPOP1 on NF- $\kappa$ B activation, neither SFV gp013L (Fig. 5

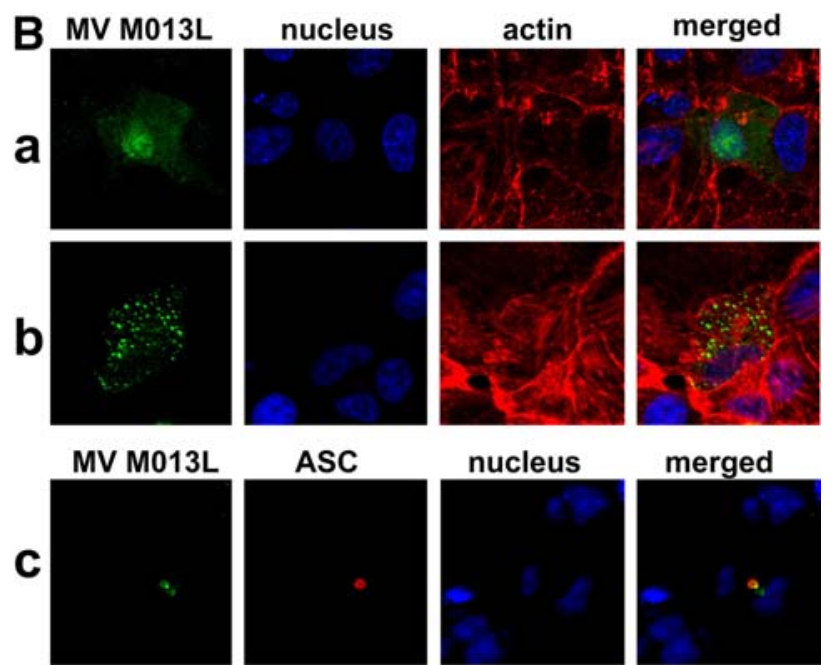

(blue), and a merged image (panel e). Twenty-four hours ( $24 \mathrm{~h})$ posttransfection cells were also infected with SFV $(\mathrm{MOI}=10)$ and cells were immunostained as described above at $48 \mathrm{~h}$ post-infection $(72 \mathrm{~h}$ post-transfection) (panel f). (B) Localization of MV M013L. Myctagged MV M013L was immunostained with a rabbit polyclonal antimyc antibody and visualized with an Alexa Fluor 488-conjugated anti-rabbit antibody $48 \mathrm{~h}$ post-transfection (panel a) and $72 \mathrm{~h}$ posttransfection (panel b). Actin was visualized with Alexa Fluor 546conjugated phalloidin and the nucleus was stained with ToPro-3. Shown is from left to right: Myc-tagged MV M013L (green), nucleus (blue), actin (red), and a merged image. Myc-tagged MV M013L and Flag-tagged ASC were immunostained $72 \mathrm{~h}$ post-transfection with rabbit polyclonal anti-myc and mouse monoclonal anti-Flag antibodies and visualized with Alexa Fluor 488 and 546-conjugated antirabbit and mouse antibodies, respectively. The nucleus was stained with ToPro-3. Shown is from left to right: Myc-tagged MV M013L (green), Flag-tagged ASC (red), nucleus (blue), and a merged image (panel c)

B) nor SFV M013L (Fig. 5C) could impair Cryopyrin (R260W) and ASC-mediated NF- $\kappa \mathrm{B}$ activation, but rather enhanced activation of NF- $\kappa$ B in a dose-dependent manner. SFV gp013L and MV M013L also enhanced TNF $\alpha$-mediated activation of NF- $\kappa \mathrm{B}$ (Fig 5D), further confirming the positive impact of $\mathrm{vPOPs}$ on the NF- $\kappa \mathrm{B}$ activation pathway. 

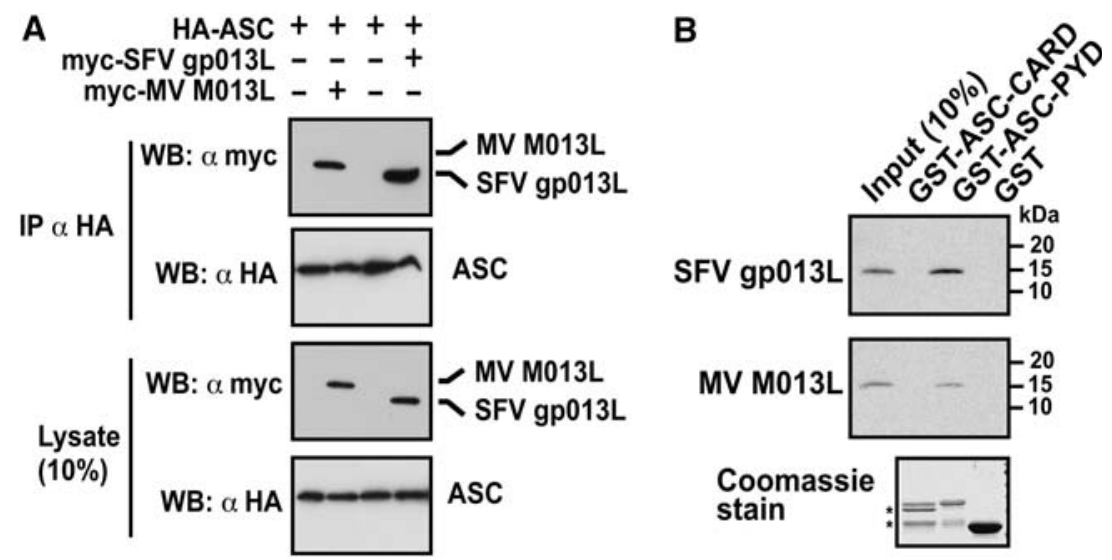

Fig. 3 SFV gp013L associates with ASC. (A) In vivo binding between ASC and SFV gp013L. HEK293T cells were transiently transfected with HA-tagged ASC, myc-tagged SFV gp013L, and myc-tagged MV M013L, as indicated. Thirty-six hours $(36 \mathrm{~h})$ posttransfection, clarified and normalized cell lysates were subjected to co-immunoprecipitation using immobilized anti-HA antibodies (Santa Cruz Biotechnology). Immune complexes were separated by SDS/ PAGE transferred onto PVDF membranes and probed with anti-myc antibodies directly conjugated to HRP. Membranes were stripped and reprobed with anti-HA-HRP antibodies. Ten percent (10\%) of the total lysate was run alongside the immunoprecipitation (IP). WB: western blot. (B) In vitro binding between ASC and SFV gp013L.
SFV gp013L and MV M013L were in vitro translated, labeled with biotin, and subjected to in vitro GST-pull down assays using GSTASC-CARD, GST-ASC-PYD, and GST immobilized to GSH sepharose, as indicated. Protein complexes were separated by SDS/ PAGE, transferred onto PVDF membranes and bound proteins were visualized by immunoblotting with streptavidin-HRP and ECL-Plus (Amersham Pharmacia Biotech) detection. A membrane is stained with coomassie blue to visualize the GST fusion proteins. An asterisk denotes two degradation products, which is present in the ASC-PYD GST fusion protein purification, but which is not affecting this assay. A molecular weight standard is indicated to the right

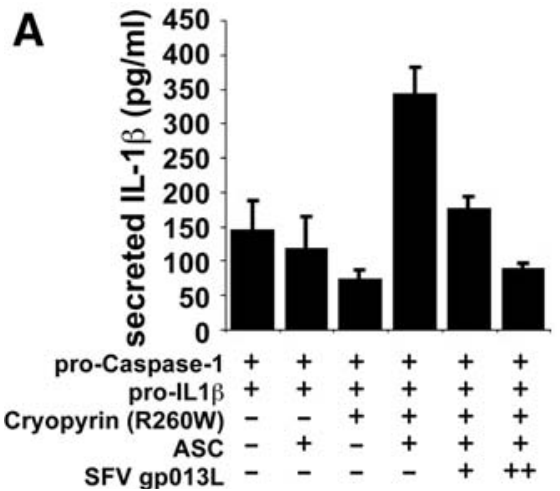

Fig. 4 SFV gp013L inhibits Caspase-1-mediated processing of proIL-1 $\beta$. HEK293N cells were transiently transfected in triplicates with expression constructs for pro-Caspase-1, murine pro-IL-1 $\beta$, Cryopyrin (R260W), ASC, and SFV gp013L (A) or MV M013L (B), as indicated. Thirty-six hours $(36 \mathrm{~h})$ post-transfection, secreted IL-1 $\beta$ was measured by ELISA (BD Pharmingen) from normalized culture supernatants using a standard curve generated with a recombinant IL$1 \beta$. Data are presented as picograms per milliliter of secreted IL- $1 \beta$

\section{Discussion}

PYD-containing proteins are important mediators of inflammatory responses during host defense. In humans, 16 potential PYD-containing pathogen recognition receptors (PYD-NLR-family members) have been recognized, including Cryopyrin. It is hypothesized that these proteins are activated by pathogen-associated molecular patterns (mean SD; $n=3$ ). (C) HEK293N cells were transfected with the indicated expression constructs using the concentrations as used for the IL- $1 \beta$ assay (A, B), and cleared protein lysates were separated by SDS/PAGE and immunoblotted with antibodies to detect expression of pro-caspase-1, Cryopyrin (R260W), ASC, SFV gp013L, and MV M013L. (+ and ++ indicate the two different expression levels of viral POPs)

(PAMPs) [1-4], and some examples of bacterial and recently viral ligands for PYD-NLRs have been specifically identified [27-31]. Activation of PYD-NLR-family proteins results in oligomerization and recruitment of the adaptor protein ASC via PYD-PYD interactions [25, 32]. Subsequently, ASC links pathogen recognition to downstream effector activation, including Caspase-1-mediated processing and secretion of IL- $1 \beta$, IL-18, and possibly 

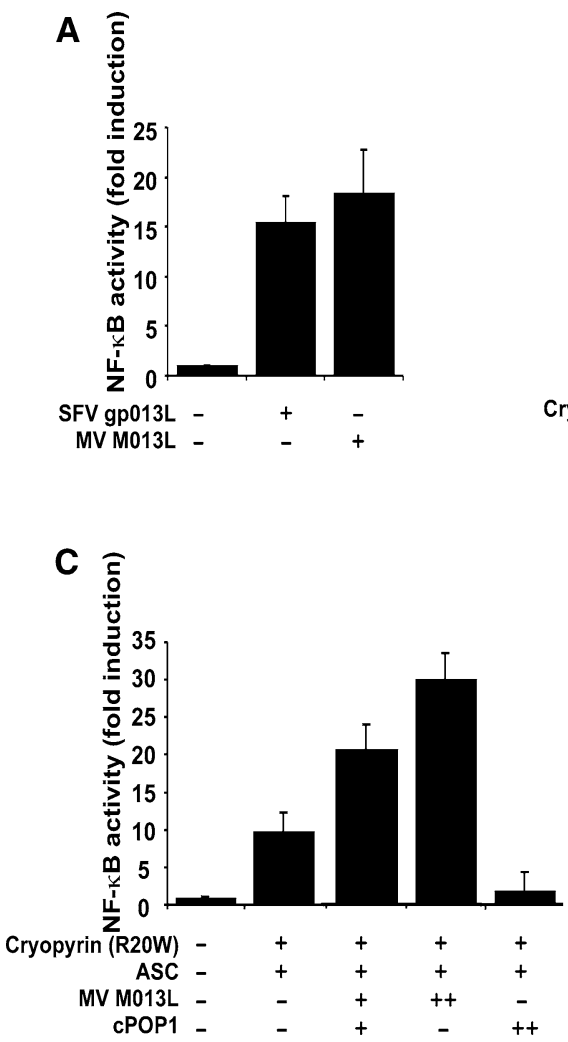

Fig. 5 SFV gp013L activates the transcription factor NF- $\kappa$ B. HEK293N cells were transiently transfected in 96-well plates in triplicates with the indicated expression constructs, including pNF$\kappa \mathrm{B}$-LUC (Stratagene) and pRL-TK (Promega), keeping the total amount of DNA constant. (A) Cells were transfected with either a control plasmid or SFV gp013L or MV M013L expression plasmids, as indicated. (B) Cells were transfected with a control plasmid or ASC and Cryopyrin (R260W) in the presence or absence of increasing amounts of SFV gp013L, or cPOP1. (C) Cells were transfected with a control plasmid or ASC and Cryopyrin (R260W) in the presence or

IL-33 [27, 33, 34]. NF- $\kappa$ B activation has been proposed as another effector of PYD signal transduction. The PYDmediated signal transduction pathway can be modulated by the cPOP1, which impairs the interaction of PYD-NLRfamily members and ASC and subsequent activation of downstream effectors [15].

In this study, we characterized a poxviral-encoded PYDcontaining protein, which is the product of S013L in the SFV genome (gp013L). As a control the product of M013L in the MV genome (M013L) was used. MV M013L has been recently shown to inhibit IL-1 $\beta$ secretion [16]. The SFV gp013L and MV M013L proteins are only 59.5\% identical (65\% conserved), but nevertheless function identically with respect to Caspase- 1 and NF- $\kappa$ B activation. Poxviruses are large dsDNA containing viruses, which are well known to encode immune evasive proteins to impair the host response upon infection [35, 36]. These immunomodulatory genes usually lack conservation be-
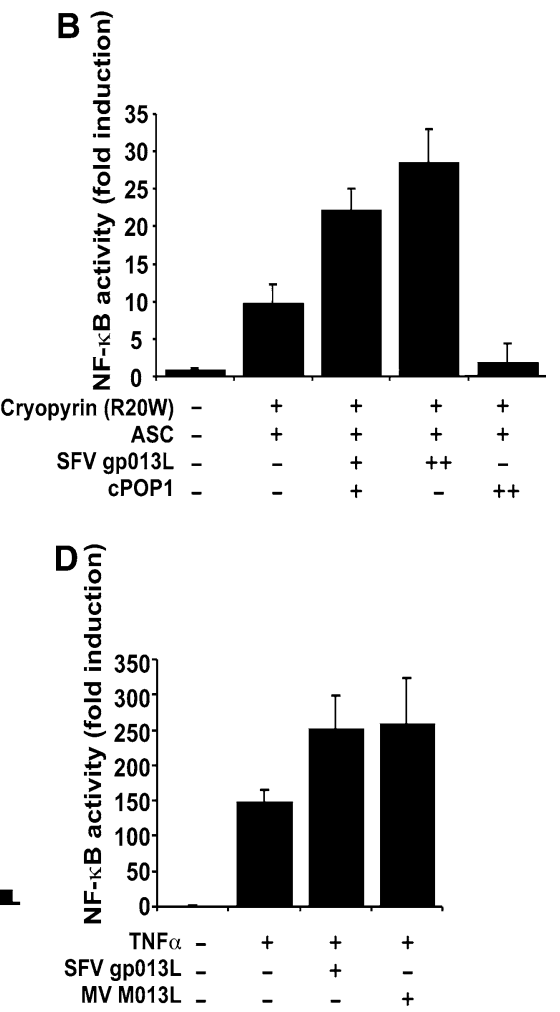

absence of increasing amounts of MV M013L, or cPOP1. (D) Cells were transfected with a control plasmid or SFV gp013L or MV M013L. Where indicated, $36 \mathrm{~h}$ post-transfection, cells were treated for $8 \mathrm{~h}$ with $20 \mathrm{ng} / \mathrm{ml}$ recombinant human TNF $\alpha$. Samples were analyzed using the Dual Glow Luciferase kit (Promega) in a Genios multimode plate reader (Tecan). Results are presented as fold induction of NF- $\kappa \mathrm{B}$ relative to control transfected cells not induced with $\mathrm{TNF} \alpha$, normalized to tymidine kinase (TK) reporter gene activity (mean SD; $n=3$ )

tween poxvirus family members, which may reflect differences in host tropism. Poxviruses are known to hijack cellular proteins that are then employed to evade immune recognition and to impair apoptosis of infected host cells. Many crucial pathways of the cellular and humoral host defense can be targeted $[35,36]$ and the fact that poxviruses also target PYD proteins further emphasizes the significance of this signal transduction pathway for host defense. SFV and MV encode inhibitors of the PYDmediated signal transduction pathway called PYD-only proteins, SFV gp013L and MV M013L, respectively. Additional members were identified in the genome of SPV, YLDV, and DpV, suggesting that these genes encode a novel class of common immune evasive proteins. To discriminate the viral and cellular POPs, we propose to rename POP1 to cPOP1. Like the cellular homolog, vPOPs interact with ASC, the central adaptor protein of the PYDsignal transduction pathway. The interaction is mediated by 
PYD-PYD, but not PYD-CARD interaction, as shown by in vitro GST pull down assays, and it appears that the binding of SFV gp013L to ASC is slightly stronger than that of MV M013L to ASC.

ASC frequently localizes to characteristic structures in the cytoplasm or forms characteristic cytoplasmic aggregates, known as specks, to which ASC-interacting proteins are frequently recruited. However, the physiological function of specks is not known. Initially vPOPs localize diffusively throughout the cell, and are found inside vesicular cytoplasmic structures $72 \mathrm{~h}$ post-transfection. Interestingly, infection of RK13 cells with SFV caused aggregation of SFV gp013L in a time-dependent manner. Co-expression with ASC caused recruitment of vPOPs into ASC-formed specks, reminiscent of cPOP1 [15]. cPOP1 localizes to the cytoplasm and the nucleus, and co-expression with ASC causes redistribution of cPOP1 into ASC formed specks [15]. Since SFV gp013L already co-localized with ASC into specks, infection of RK13 cells with SFV did not alter their localization.

The best-characterized PYD-activated downstream effector is Caspase-1. Activation of Caspase-1 is required for the processing, activation and subsequent secretion of the proinflammatory cytokines pro-IL- $1 \beta$, pro-IL-18, and pro-IL-33 [37, 38]. Activation of Caspase-1 requires prior activation of PYD-NLR-family proteins by their cognate ligands, subsequent recruitment of ASC, and formation of inflammasomes. Both SFV gp013L and MV M013L impaired Cryopyrin and ASC-mediated activation of Caspase-1, which might be explained by the impaired formation of an inflammasome containing ASC and Cryopyrin in the presence of vPOPs. The ability of vPOPs to impair Caspase-1 activation in response to other than Cryopyrin containing inflammasomes will need further investigation. Apart from vPOPs, poxviruses are known to encode other proteins that interfere with the Caspase-1-IL-1 $\beta$ pathway. Several poxviruses encode soluble IL-1 receptors, which bind only to IL-1 $\beta$. The cellular receptor, however, binds IL- $1 \alpha$, IL- $1 \beta$ and IL-1RA, suggesting that specific inhibition of IL- $1 \beta$ is crucial during poxvirus infection $[35,36]$. MV and SFV encode Serp2, which shows similarity to the cowpox virus crmA, and also inhibits Caspase-1 [35, 36]. Poxviruses acquired mechanisms to impair the generation and function of IL- $1 \beta$ at three different levels: (1) at the inflammasome formation and signal transduction leading to Caspase- 1 activation, (2) directly at Caspase- 1 activation, and (3) at the binding of IL- $1 \beta$ to its receptor. Blocking three key steps by three distinct poxviral-encoded proteins emphasizes the importance of this cytokine for host defense. Poxviruses also encode proteins, such as IL18BPs, to interfere with IL-18, a cytokine that also requires Caspase-1 for processing [35, 36].
Expression of vPOPs strongly promoted activation of $\mathrm{NF}-\kappa \mathrm{B}$, which is in contrast to cPOP1 [15]. POPs most likely impair the PYD-PYD association between ASC and PYD-NLR-family proteins, thereby disrupting the pathway leading to the activation of these effectors, which occurs in the inflammasome. It is therefore surprising that $\mathrm{NF}-\kappa \mathrm{B}$ activation is not impaired by vPOPs, suggesting selectivity for Caspase-1 activation. We recently demonstrated that ASC can compete with Cardiak (Rip2) for pro-Caspase-1 binding [18], and a recent study suggested that ASC interaction with Caspase-1 prevents the interaction of Caspase-1 with Cardiak, and that this interaction is required for the activation of $\mathrm{NF}-\kappa \mathrm{B}$ [39]. According to this model, the disruption of the PYD-NLR-ASC interaction by vPOPs might also impair the subsequent interaction of ASC with pro-Caspase-1. As a result, pro-Caspase-1 would be available to associate with Cardiak to activate NF- $\kappa \mathrm{B}$. Upon infection, poxviruses, including the MV, potentially block the NF- $\kappa \mathrm{B}$ activation pathway using virus-encoded conserved ankyrin-containing proteins, such as the critical virulence factor M150R (MNF), which function analogous to cellular $\mathrm{I} \kappa \mathrm{Bs}$ [40]. PYD proteins link to the $\mathrm{NF}-\kappa \mathrm{B}$ activation pathway at the level of the IKK complex, and also Caspase- 8 has been implicated in this pathway $[17,26$, $41,42]$. However, many viruses activate NF- $\kappa \mathrm{B}$, which establishes a more efficient infection, which promotes viral replication and prevents virus-induced apoptosis of infected cells [43]. High and constitutive activation of NF$\kappa \mathrm{B}$ has also been linked to tumorigenesis, and $\mathrm{MV}$ and SFV are tumorigenic in their natural hosts, and activation of $\mathrm{NF}-\kappa \mathrm{B}$ by virulence factors could support tumor development by promoting proliferation and impairing the apoptotic host response [44].

Cryopyrin, one of the PYD-NLR-family proteins for which ligands have been identified, senses several bacterial-derived PAMPs [27-30]. A very recent report also provides evidence that Cryopyrin is required for Caspase-1 activation in response to viral infections and recognizes viral-derived PAMPs, such as dsRNA [31]. The observation that poxviruses encode immune evasive proteins that target the PYD-signal transduction pathway, further support that viral PAMPs might function as ligands for some PYD-NLRs. In summary, our data provide evidence for the existence of a SFV-encoded gp013L, which in spite of the rather low sequence similarity compared to the MV-encoded M013L, functions identically by inhibiting activation of Caspase- 1 and activating NF- $\kappa \mathrm{B}$. Virus-encoded PYD proteins represent a novel class of conserved immune evasive proteins, which target the host inflammasome. This observation further emphasizes the importance of PYD proteins for host defense and support that viral-derived PAMPs are recognized by the inflammasome. 
Acknowledgments The project described was supported by Grant Number 5P20-RR-016440 (CS, DCF) from the National Center for Research Resources (NCRR), a component of the National Institutes of Health (NIH) and Grant Numbers 1R21-AI-067680 (CS), 1R03AI-067806 (CS), and R 01-AI-56324 (JCR) from NIAID/NIH. Its contents are solely the responsibility of the authors and do not necessarily represent the official views of NCRR or NIH. We are also grateful to the support from the Alexander Bland Osborn Cancer Center Endowment (CS).

\section{References}

1. C. Stehlik, J.C. Reed, J. Exp. Med. 200, 551-558 (2004)

2. J. Tschopp, F. Martinon, K. Burns, Nat. Rev. Mol. Cell Biol. 4, 95-104 (2003)

3. J.P. Ting, B.K. Davis, Annu. Rev. Immunol. 23, 387-414 (2004)

4. N. Inohara, G. Nunez, Nat. Rev. Immunol. 3, 371-381 (2003)

5. K.E. Conway, B.B. McConnell, C.E. Bowring, C.D. Donald, S.T. Warren, P.M. Vertino, Cancer Res. 60, 6236-6242 (2000)

6. J. Masumoto, S. Taniguchi, K. Ayukawa, H. Sarvotham, T. Kishino, N. Niikawa, E. Hidaka, T. Katsuyama, T. Higuchi, J. Sagara, J. Biol. Chem. 274, 33835-33838 (1999)

7. F. Martinon, K. Burns, J. Tschopp, Mol. Cell. 10, 417-426 (2002)

8. S. Brydges, D.L. Kastner, Curr. Top. Microbiol. Immunol. 305, 127-160 (2006)

9. S.-H. Lee, C. Stehlik, J.C. Reed, J. Biol. Chem. 276, 34495$34500(2001)$

10. E.W. Humke, S.K. Shriver, M.A. Starovasnik, W.J. Fairbrother, V.M. Dixit, Cell 103, 99-111 (2000)

11. A. Druilhe, S.M. Srinivasula, M. Razmara, M. Ahmad, E.S. Alnemri, Cell Death Differ. 8, 649-657 (2001)

12. M. Lamkanfi, G. Denecker, M. Kalai, K. D’Hondt, A. Meeus, W. Declercq, X. Saelens, P. Vandenabeele, J. Biol. Chem. 279, 51729-51738 (2004)

13. G. Condorelli, G. Vigliotta, A. Cafieri, A. Trencia, P. Andalo, F. Oriente, C. Miele, M. Caruso, P. Formisano, F. Beguinot, Oncogene 18, 4409-4415 (1999)

14. A. Krueger, I. Schmitz, S. Baumann, P.H. Krammer, S. Kirchhoff, J. Biol. Chem. 276, 20633-20640 (2001)

15. C. Stehlik, M. Krajewska, K. Welsh, S. Krajewski, A. Godzik, J.C. Reed, Biochem. J. 373, 101-113 (2003)

16. J.B. Johnston, J.W. Barrett, S.H. Nazarian, M. Goodwin, D. Ricuttio, G. Wang, G. McFadden, Immunity 23, 587-598 (2005)

17. C. Stehlik, L. Fiorentino, A. Dorfleutner, J.M. Bruey, E.M. Ariza, J. Sagara, J.C. Reed, J. Exp. Med. 196, 1605-1615 (2002)

18. C. Stehlik, S.H. Lee, A. Dorfleutner, A. Stassinopoulos, J. Sagara, J.C. Reed, J. Immunol. 171, 6154-6163 (2003)

19. E. Liepinsh, R. Barbals, E. Dahl, A. Sharipo, E. Staub, G. Otting, J. Mol. Biol. 332, 1155-1163 (2003)

20. C. Cameron, S. Hota-Mitchell, L. Chen, J. Barrett, J.X. Cao, C. Macaulay, D. Willer, D. Evans, G. McFadden, Virology 264, 298-318 (1999)

21. D.O. Willer, G. McFadden, D.H. Evans, Virology 264, 319-343 (1999)

22. C.L. Afonso, E.R. Tulman, Z. Lu, L. Zsak, F.A. Osorio, C. Balinsky, G.F. Kutish, D.L. Rock, J. Virol. 76, 783-790 (2002)
23. H.J. Lee, K. Essani, G.L. Smith, Virology 281, 170-192 (2001)

24. C.L. Afonso, G. Delhon, E.R. Tulman, Z. Lu, A. Zsak, V.M. Becerra, L. Zsak, G.F. Kutish, D.L. Rock, J. Virol. 79, 966-977 (2005)

25. T.A. Dowds, J. Masumoto, L. Zhu, N. Inohara, G. Nunez, J. Biol. Chem. 279, 21924-21928 (2004)

26. G.A. Manji, L. Wang, B.J. Geddes, M. Brown, S. Merriam, A. Al-Garawi, S. Mak, J.M. Lora, M. Briskin, M. Jurman, J. Cao, P.S. DiStefano, J. Bertin, J. Biol. Chem. 277, 11570-11575 (2002)

27. F.S. Sutterwala, Y. Ogura, M. Szczepanik, M. Lara-Tejero, G.S. Lichtenberger, E.P. Grant, J. Bertin, A.J. Coyle, J.E. Galan, P.W. Askenase, R.A. Flavell, Immunity 24, 317-327 (2006)

28. T.D. Kanneganti, N. Ozoren, M. Body-Malapel, A. Amer, J.H. Park, L. Franchi, J. Whitfield, W. Barchet, M. Colonna, P. Vandenabeele, J. Bertin, A. Coyle, E.P. Grant, S. Akira, G. Nunez, Nature 440, 232-236 (2006)

29. F. Martinon, V. Petrilli, A. Mayor, A. Tardivel, J. Tschopp, Nature 440, 237-241 (2006)

30. S. Mariathasan, D.S. Weiss, K. Newton, J. McBride, K. O'Rourke, M. Roose-Girma, W.P. Lee, Y. Weinrauch, D.M. Monack, V.M. Dixit, Nature 440, 228-232 (2006)

31. T.D. Kanneganti, M. Body-Malapel, A. Amer, J.H. Park, J. Whitfield, Z.F. Taraporewala, D. Miller, J.T. Patton, N. Inohara, G. Nunez, J. Biol. Chem.281, 36560-36568 (2006)

32. J.W. Yu, J. Wu, Z. Zhang, P. Datta, I. Ibrahimi, S. Taniguchi, J. Sagara, T. Fernandes-Alnemri, E.S. Alnemri, Cell Death Differ. 13, 236-249 (2006)

33. S. Mariathasan, K. Newton, D.M. Monack, D. Vucic, D.M. French, W.P. Lee, M. Roose-Girma, S. Erickson, V.M. Dixit, Nature 430, 213-218 (2004)

34. M. Yamamoto, K. Yaginuma, H. Tsutsui, J. Sagara, X. Guan, E. Seki, K. Yasuda, M. Yamamoto, S. Akira, K. Nakanishi, T. Noda, S. Taniguchi, Genes Cells 9, 1055-1067 (2004)

35. B.T. Seet, J.B. Johnston, C.R. Brunetti, J.W. Barrett, H. Everett, C. Cameron, J. Sypula, S.H. Nazarian, A. Lucas, G. McFadden, Annu. Rev. Immunol. 21, 377-423 (2003)

36. B. Moss, J.L. Shisler, Semin. Immunol. 13, 59-66 (2001)

37. G. Fantuzzi, C.A. Dinarello, J. Clin. Immunol. 19, 1-11 (1999)

38. J. Schmitz, A. Owyang, E. Oldham, Y. Song, E. Murphy, T.K. McClanahan, G. Zurawski, M. Moshrefi, J. Qin, X. Li, D.M. Gorman, J.F. Bazan, R.A. Kastelein, Immunity 23, 479-490 (2005)

39. A. Sarkar, M. Duncan, J. Hart, E. Hertlein, D.C. Guttridge, M.D. Wewers, J. Immunol. 176, 4979-4986 (2006)

40. C. Camus-Bouclainville, L. Fiette, S. Bouchiha, B. Pignolet, D. Counor, C. Filipe, J. Gelfi, F. Messud-Petit, J. Virol. 78, 2510 2516 (2004)

41. L. Wang, G.A. Manji, J.M. Grenier, A. Al-Garawi, S. Merriam, J.M. Lora, B.J. Geddes, M. Briskin, P.S. DiStefano, J. Bertin, J. Biol. Chem. 277, 29874-29880 (2002)

42. M. Hasegawa, R. Imamura, T. Kinoshita, N. Matsumoto, J. Masumoto, N. Inohara, T. Suda, J. Biol. Chem. 280, 15122-15130 (2005)

43. J. Hiscott, H. Kwon, P. Genin, J. Clin. Invest. 107, 143-151 (2001)

44. M. Karin, Y. Cao, F.R. Greten, Z.-W. Li, Nat. Rev. Cancer 2, 301-310 (2002) 


\title{
Cellular Pyrin Domain-Only Protein 2 Is a Candidate Regulator of Inflammasome Activation ${ }^{\nabla}$
}

\author{
Andrea Dorfleutner,${ }^{1} \dagger$ Nicole B. Bryan, ${ }^{1} \dagger$ Siera J. Talbott,${ }^{1}$ Kristin N. Funya, ${ }^{1}$ Stephanie L. Rellick, ${ }^{1}$ \\ John C. Reed, ${ }^{2}$ Xianglin Shi, ${ }^{3}$ Yon Rojanasakul, ${ }^{4}$ Daniel C. Flynn, ${ }^{1}$ and Christian Stehlik ${ }^{1 *}$ \\ Mary Babb Randolph Cancer Center and Department of Microbiology, Immunology \& Cell Biology, West Virginia University School of \\ Medicine, 1 Medical Center Drive, Morgantown, West Virginia 26506-9300 ${ }^{1}$; Burnham Institute for Medical Research, \\ 10901 North Torrey Pines Road, La Jolla, California 92037²; Pathology and Physiology Research Branch, \\ Health Effects Laboratory Division, National Institute for Occupational Safety and Health, Morgantown, \\ West Virginia $26505^{3}$; and Department of Pharmaceutical Sciences, West Virginia University \\ School of Pharmacy, 1 Medical Center Drive, Morgantown, West Virginia 26506-9500 4
}

Received 15 August 2006/Returned for modification 23 September 2006/Accepted 4 December 2006

\begin{abstract}
Pyrin domain (PYD) proteins have recently emerged as important signaling molecules involved in the development of innate immunity against intracellular pathogens through activation of inflammatory mediator pathways. ASC is the central adaptor protein, which links pathogen recognition by PYD-containing pathogen recognition receptors, known as PYD-Nod-like receptors (NLR), PAN, PYPAF, NALP, Nod, and Caterpiller proteins, to the activation of downstream effectors, including activation of caspase-1 and NF- $\mathrm{B}$. Activation of these effectors occurs when specific protein complexes, known as inflammasomes, are formed. PYD signal transduction leads to inflammasome assembly and activation of specific effector proteins. It is modulated by a cellular PYD-only protein (cPOP1), which binds to ASC and interferes with the recruitment of ASC to activated PYD-NLRs. Here we describe the identification and characterization of a second cellular POP (cPOP2), which shows highest homology to the PYD of PAN1. cPOP2 binds to ASC and PAN1, thereby blocking formation of cryopyrin and PAN1-containing inflammasomes, activation of caspase-1, and subsequent processing and secretion of bioactive interleukin-1 $\beta$. Existence of a second cPOP provides additional insights into inflammasome formation and suggests that POPs might be a common regulatory mechanism to "fine-tune" the activity of specific PYD-NLR family protein-containing inflammasomes.
\end{abstract}

Pyrin domain (PYD) proteins are important signaling molecules involved in host defense against pathogens through activation of inflammatory mediator pathways. Humans have at least 23 proteins containing a PYD $(12,31,33,34)$. Pathogens are recognized by the leucine-rich region (LRR) of PYDcontaining pathogen recognition receptors, known as PYDNod-like receptors (NLR), pyrin- and NACHT domain-containing proteins (PAN), pyrin domain-containing Apaf1-like proteins (PYPAF), NACHT-LRR- and pyrin domain-containing proteins (NALP), nucleotide-binding oligomerization domain-containing proteins (Nod), and Caterpiller proteins. Activation of these proteins is hypothesized to be achieved through displacement of the intramolecular interaction of the LRR with the NACHT (NAIP, CIITA, HET-E, and TP1) domain. Activated PYD-NLR family proteins recruit and oligomerize the PYD-containing adaptor protein ASC (apoptosis-associated speck-like protein containing a caspace recruitment domain; TMS1) $(5,24)$ into cytoplasmic structures, referred to as the inflammasomes, where downstream effector activation occurs (22). Caspase-1-mediated processing of prointerleukin (IL)-1 $\beta$ and activation of the transcription factor

\footnotetext{
* Corresponding author. Mailing address: Mary Babb Randolph Cancer Center and Department of Microbiology, Immunology \& Cell Biology, West Virginia University School of Medicine, 2826 MBRCC, 1 Medical Center Drive, Morgantown, WV 26506-9300. Phone: (304) 293-8785. Fax: (304) 293-4667. E-mail: cstehlik@hsc.wvu.edu.

$\dagger$ These authors contributed equally to this work.

${ }^{\nabla}$ Published ahead of print on 18 December 2006.
}

NF-кB have been described as effectors of PYD-mediated signal transduction, and enforced oligomerization of ASC is sufficient for their activation.

Hereditary mutations in PYD family proteins can cause dysregulated recruitment of ASC, which leads to autoinflammatory disorders, such as periodic fever syndromes. Specifically, mutations in pyrin (marenostrin) account for familial Mediterranean fever, whereas mutations in the PYD-NLR family member cryopyrin (CIAS1, PYPAF1, NALP3, CLR1.1) have been linked to familial cold autoinflammatory syndrome, Muckle-Wells syndrome, and chronic infantile neurological cutaneous and articular syndrome (3).

The PYD belongs to the death domain fold (DDF) domain family, which also includes the caspase recruitment domain (CARD), the death domain (DD), and the death effector domain (DED). CARD and DED interactions are also regulated by CARD-only proteins (COPs) and DED-only proteins, respectively. These small proteins are composed of a single CARD or one or two DED domains and include the CARDonly proteins COP (Pseudo-ICE), ICEBERG and INCA (9, $11,17,18)$, or the DED-only proteins PED (PEA-15), FLIP-s, and viral FLIP $(4,16)$. In general, DDF-only proteins can function as dominant-negative inhibitors for particular signaling pathways by competing for critical binding partners, thereby interrupting signal transmission to downstream effector proteins. Inflammasome formation, upon pathogen infection, is regulated by cellular and viral pyrin-only proteins (vPOPs), which interfere with the PYD-PYD interaction between ASC and PYD-NLR family proteins, thereby impairing 
host defense by blocking downstream effectors (13, 29; A. Dorfleutner, S. J. McDonald, N. B. Bryan, K. N. Funya, J. C. Reed, X. Shi, D. C. Flynn, Y. Rojanasakul, and C. Stehlik, submitted for publication). Here we report the identification and characterization of a second cellular POP, cPOP2, which impairs PYD-mediated activation of pro-caspase-1 and subsequent processing of pro-IL-1 $\beta$.

\section{MATERIALS AND METHODS}

Plasmids and strains. The complete open reading frame of cPOP2 was cloned from a pooled cDNA library by high-fidelity PCR (PfuUltra; Stratagene) using the primer pair 5'-GCGAATTCATGGCATCTTCTGCAGAGCTG-3' and 5'CCGCCTCGAGTTAAGGTGGGGGCATCACACA- $3^{\prime}$. The PCR product was digested with EcoRI and XhoI restriction enzymes and cloned into pcDNA3 (Invitrogen) expression plasmids, which were modified to contain an $\mathrm{NH}_{2}$-terminal myc, hemagglutinin (HA), or Flag epitope tag. The authenticity of cPOP2 was confirmed by DNA sequence analysis. Expression constructs for ASC, ASCPYD, ASC-CARD, cPOP1, cryopyrin (R260W), PAN1, pro-IL-1 $\beta$, and procaspase-1 were described previously $(2,28-30)$.

Cell culture and transfection. HEK293N, HEK293T, and Jurkat cells were obtained from the American Type Culture Collection and cultured in Dulbecco's modified Eagle's medium (HEK293) and RPMI 1640 medium (Jurkat cells) and supplemented with $4 \mathrm{mM}$ L-glutamine, $1.5 \mathrm{~g}$ /liter sodium bicarbonate, $0.1 \mathrm{mM}$ nonessential amino acids, $1.0 \mathrm{mM}$ sodium pyruvate, and $10 \%$ fetal bovine serum. HEK293 cells were transfected using Polyfect (QIAGEN) or the calcium precipitation method, and Jurkat cells were transfected using Lipofectamine Plus (Invitrogen).

Reverse transcriptase PCR (RT-PCR) reaction. RNA was isolated from cells using the TRIzol reagent (Invitrogen), according to the manufacturer's instructions. Total RNA (5 $\mu \mathrm{g})$ was subjected to DNase I (Invitrogen) treatment and reverse transcribed using the Superscript II enzyme (Invitrogen) into first-strand cDNA, as suggested by the manufacturer, followed by PCR amplification with GoTaq polymerase (Promega) using the cPOP2-specific primer pair 5'-ATGG CATCTTCTGCAGAGCTG-3' and 5'-TTAAGGTGGGGGCATCACACA-3' $(294 \mathrm{bp})$ and the $\beta$-actin-specific primer pair $5^{\prime}$-GACGATGATATTGCCGCA CT-3', 5'-GATACCACGCTTGCTCTGAG-3' (533 bp). A negative-control experiment with pooled RNA samples, in which the RT was omitted during the RT-PCR step, was also performed. PCR products were excised from agarose gels, and sequences were verified.

Coimmunoprecipitation assay. Immunoprecipitation assays were performed following transient expression of the HA-tagged PYD of ASC (ASC-PYD) and the myc-tagged cPOP2; the myc-tagged PYD of PAN1 (PAN1-PYD) and the Flag-tagged cPOP2; and PAN1 and the myc-tagged cPOP2 into HEK293T cells. At $36 \mathrm{~h}$ posttransfection, cells were lysed in isotonic lysis buffer $(150 \mathrm{mM} \mathrm{NaCl}$, $20 \mathrm{mM}$ Tris- $\mathrm{HCl}$ [pH 7.4], 10\% glycerol, $0.2 \%$ NP-40) supplemented with protease and phosphatase inhibitors. Clarified lysates were subjected to immunoprecipitation using agarose-conjugated anti-myc or anti-Flag antibodies (Santa Cruz Biotechnology) for $4 \mathrm{~h}$ at $4^{\circ} \mathrm{C}$. Following extensive washing in lysis buffer, bound immune complexes were separated by sodium dodecyl sulfate-polyacrylamide gel electrophoresis (SDS-PAGE) and analyzed by immunoblotting using horseradish peroxidase (HRP)-conjugated anti-HA (Santa Cruz Biotechnology) and anti-myc (Santa Cruz Biotechnology) antibodies or anti-PAN1 antibodies (Imgenex) in conjunction with an ECL detection system (Amersham-Pharmacia). Where indicated, cell lysates (5\% volume) were included alongside immune complexes. Alternatively, lysates were directly analyzed by immunoblotting after normalization for total protein content.

In vitro protein interaction assay. cPOP2 was expressed as a glutathione $S$-transferase (GST) fusion protein in Escherichia coli BL-21 cells (Stratagene) and affinity purified using glutathione (GSH)-Sepharose (Amersham-Pharmacia). GST-cPOP2 or the GST control (50 ng) immobilized on $10 \mu \mathrm{l} \mathrm{GSH}$ Sepharose was blocked in a mixture containing $142.4 \mathrm{mM} \mathrm{KCl}, 5 \mathrm{mM} \mathrm{MgCl}, 10$ mM HEPES (pH 7.4), 0.5 mM EGTA, 1 mM EDTA, 0.2\% Nonidet P-40, and 1 $\mathrm{mM}$ dithiothreitol and supplemented with protease inhibitors and $1 \mathrm{mg} / \mathrm{ml}$ bovine serum albumin (BSA) for $30 \mathrm{~min}$ at room temperature. Beads were washed twice and incubated with in vitro-translated and biotinylated (Promega) ASC and PAN1-PYD proteins overnight at $4^{\circ} \mathrm{C}$ as described above, except that the buffer was supplemented with protease inhibitors and $0.5 \mathrm{mg} / \mathrm{ml}$ BSA. Bound proteins were washed extensively, separated by SDS-PAGE, immunoblotted with streptavidin-HRP, and detected with ECL-Plus (Amersham-Pharmacia).
Direct yeast two-hybrid interaction. The EGY48 strain of Saccharomyces cerevisiae (MAT trp1 ura3 his leu2::plexApo6-leu2) was maintained in yeast extract-peptone-dextrose medium. cPOP2 and the PYD of several NLR family proteins, pyrin, and ASC were cloned into pJG4-5 and pGilda yeast expression vectors under the GAL1 promoter. Plasmids were cotransformed by the $\mathrm{LiCl}$ method with pSH1834, which contains eight repeats of a LexA promoter to drive expression of the $\beta$-galactosidase reporter gene, in EGY48. Transformed yeast cells were plated onto leucine-deficient Burkholder's minimal medium containing $2 \%$ galactose and $1 \%$ raffinose and supplemented with appropriate amino acids, and the interaction of the proteins was scored by leucine complementation-dependent growth. Colonies were then replicated onto Burkholder's minimal medium plates containing leucine and $2 \%$ glucose, and $\beta$-galactosidase filter assays were performed and scored according to the time required to yield a blue color.

Confocal microscopy analysis. HEK293T cells were seeded onto collagen type I-coated coverslips in six-well dishes and transiently transfected the following day with pcDNA3 expression plasmids for myc-tagged cPOP2, myc-tagged cPOP1, Flag-tagged ASC, and HA-tagged PAN1 as indicated, using the calcium phosphate precipitation method. At $36 \mathrm{~h}$ posttransfection, cells were fixed in $3.7 \%$ formaldehyde; permeabilized with $0.5 \%$ saponin (Sigma); blocked with $0.5 \%$ saponin, $1.5 \%$ BSA, and $1.5 \%$ normal goat serum (Zymed); and immunostained with rabbit polyclonal anti-myc antibodies (1:500; Santa Cruz Biotech), mouse monoclonal anti-Flag M2 antibodies (1:3,500, Sigma), rat polyclonal anti-HA antibodies (1:2,500; Roche Applied Science), and secondary Alexa Fluor 488 and 546 conjugated antibodies (1:200; Molecular Probes) in phosphate-buffered saline supplemented with $0.5 \%$ saponin, $1.5 \%$ BSA, and $1.5 \%$ normal goat serum (Zymed). Nuclei were visualized by incubation with ToPro-3 (Molecular Probes), and the actin cytoskeleton was stained with Alexa Fluor 488- or 546-conjugated phalloidin. After three washes with PBS, supplemented with $0.5 \%$ saponin, samples were mounted with Vectashield (Vectorlabs), and images were acquired by confocal laser-scanning microscopy (Zeiss LSM510).

IL-1及 secretion assay. HEK293N cells were transiently transfected with pcDNA3 expression plasmids for mouse pro-IL-1 $\beta$, myc-tagged pro-caspase-1, HA-tagged ASC, myc-tagged cryopyrin, untagged PAN1, and Flag-tagged cPOP2 in 24-well plates using the Polyfect transfection reagent (QIAGEN). At $24 \mathrm{~h}$ posttransfection, the culture medium was replenished with $0.5 \mathrm{ml}$ of fresh culture medium. At $36 \mathrm{~h}$ posttransfection, IL-1 $\beta$ secreted into the culture supernatants was measured by enzyme-linked immunosorbent assay (ELISA) using a commercial kit (BD Biosciences-Pharmingen) and a Genios multimode plate reader (Tecan). Assays were performed in triplicate, and data were normalized for cell number, as determined by crystal violet assay.

\section{RESULTS}

Identification of a second cellular PYD-only protein. We recently characterized human POP1, which is highly similar to the PYD of ASC, and showed that it interferes with the PYDPYD interaction between ASC and cryopyrin to block activation of NF-кB (29). POP1 represents the first endogenous regulator of the PYD signal transduction pathway. More recently, we and others identified poxviral POP homologues that impair host defense by preventing inflammasome formation, which prompted us to suggest renaming POP1 to cPOP1 ( $7 \mathrm{a}$, 13). Poxviruses are large double-stranded DNA viruses, which encode virulence factors that enable them to evade the host immune responses upon infection, and are known to frequently hijack proteins from the host $(25,27)$.

We mined the human genome for additional cellular POPs. Since cPOP1 is most homologous to the PYD of ASC (29), we applied the PYD of other PYD-containing proteins for the database mining. Using the PYD of PAN1 (PYPAF2, NALP2, NBS1, CLR19.9) (a PYD-NLR protein), we identified a potential PYD-containing open reading frame, carried on a single exon on chromosome $3 \mathrm{q} 28$, which recently was also predicted by NCBI using GNOMON (accession no. XR_001234). The deduced amino acid sequence revealed a potential POP, similar to cPOP1 and several poxviral POPs, which all bind to ASC 

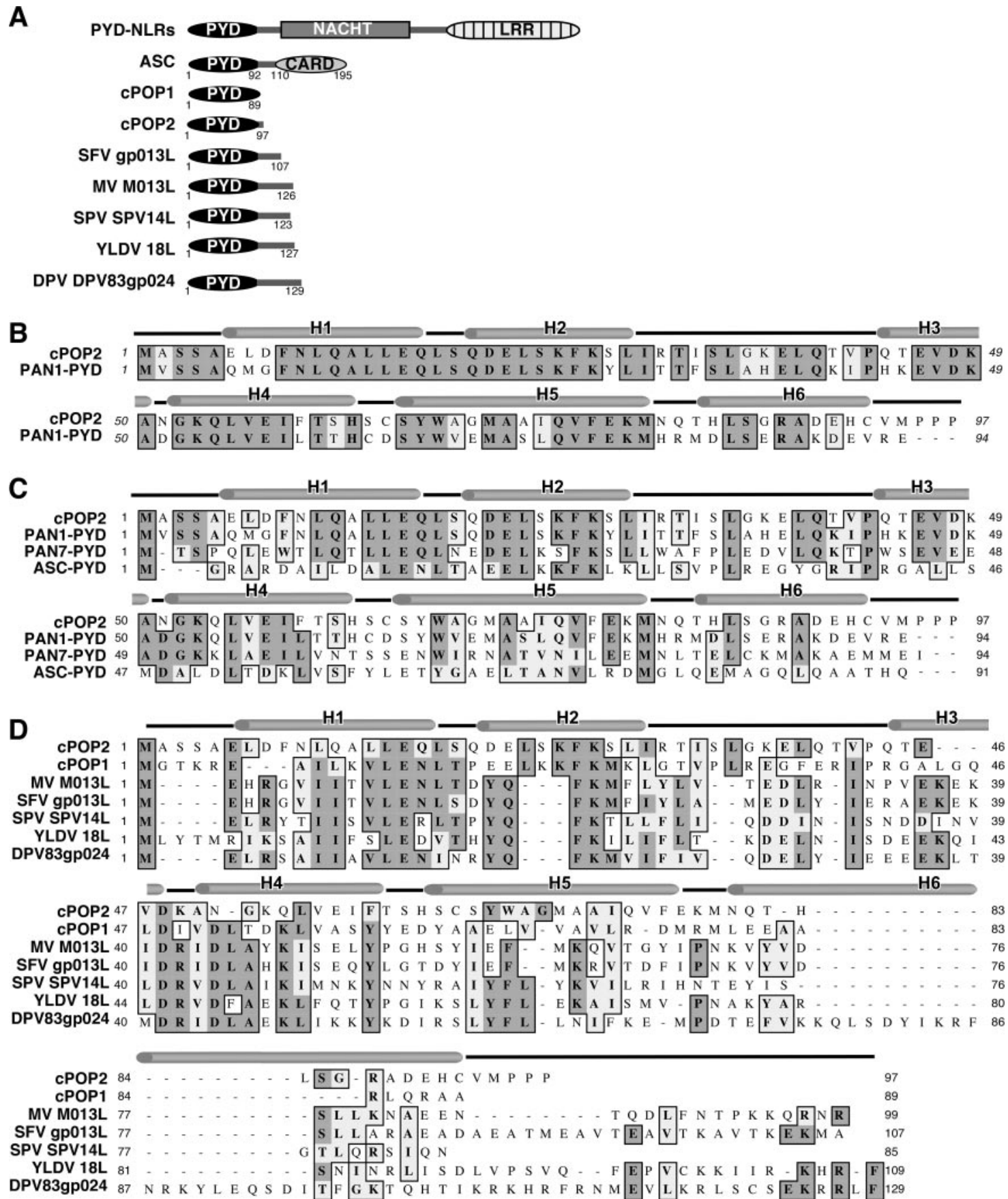

FIG. 1. The human genome encodes a second cellular PYD-only protein. (A) Schematic domain representation of cPOP2 and other known viral and cellular POPs, ASC, and PYD-NLR family proteins. Myxoma virus (MV; M013L), rabbit Shope fibroma virus (SFV; gp013L), swinepox virus (SPV; SPV14L), Yaba-like disease virus (YLDV; 18L), mule deer poxvirus (DPV; DPV83gp024). (B) Clustal W alignment of cPOP2 and the most similar PYD of PAN1 (PYPAF2, NALP2, NBS1, CLR19.9). (C) cPOP2 and the PYDs of the three most similar PYD-containing proteins, PAN1, PAN 7 (PYPAF3, NALP7, NOD12, CLR19.4), and ASC. (D) CPOP2 with other known POPs. Dark gray and light gray shading indicates identical and similar (conserved) amino acid residues, respectively. The $\alpha$-helices, as determined for the PYD of ASC, are marked above the sequences (e.g., H1, H2, etc.) (19).

by PYD-PYD interaction (Fig. 1A). This protein, which we named cPOP2, shows $68 \%$ sequence similarity (61\% sequence identity) to the PYD of PAN1 (Fig. 1B). High sequence similarity of cPOP2 to the PYD of PAN7 (PYPAF3, NALP7, NOD12, CLR19.4) and ASC (Fig. 1C) is also detectable. Sev- eral conserved amino acid residue patches are present in all known POPs (Fig. 1D).

cPOP2 is expressed in human cells. Because we predicted cPOP2 from the human genome, we investigated whether cPOP2 is actually expressed in human cells. Due to the lack of 

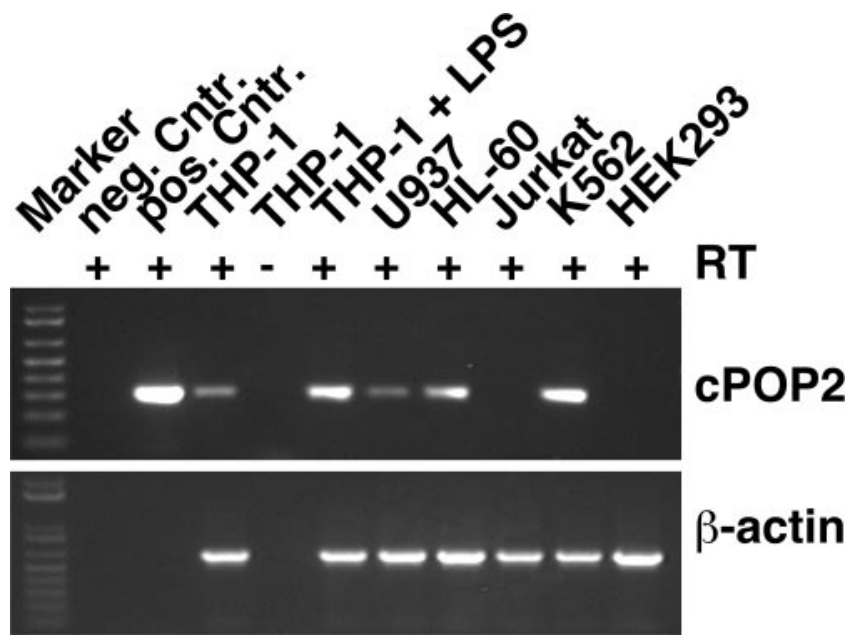

FIG. 2. cPOP2 is expressed in human cell lines. cPOP2-specific transcripts were detected in several human cell lines by RT-PCR, which was performed with cPOP2-specific primers and primers specific for $\beta$-actin. RT-PCR was also performed using pooled RNA from THP-1 cells treated with $600 \mathrm{ng} / \mathrm{ml}$ LPS (E. coli serotype O111:B4) for 2, 4, 6, 12, and $24 \mathrm{~h}$ (THP-1 + LPS). A cPOP1 expression plasmid (pcDNA3-myc-cPOP1) was included as a negative control, and a cPOP2 expression plasmid (pcDNA3-myc-cPOP2) was used as a positive control. Pooled RNA not incubated with reverse transcriptase during the RT-PCR step was also used as a negative control.

a specific antibody, we performed RT-PCR analysis using cPOP2-specific primers and RNA isolated from various human cell lines. The presence of a cPOP2-specific transcript of 294 bp in several cell lines confirmed that $\mathrm{cPOP} 2$ is indeed transcribed and that it encodes a predicted protein of approximately $11 \mathrm{kDa}$ (Fig. 2). Since cPOP2 is encoded on a single exon, we ensured that all potential genomic DNA contamination of the isolated RNA was removed by treatment of the RNA with DNase I, prior to cDNA synthesis. We also performed a negative-control cDNA synthesis reaction from THP-1 RNA in the absence of the RT, which did not yield a cPOP2-specific PCR product, verifying that no genomic DNA contamination had occurred. We performed a PCR using as a template a plasmid-encoding cPOP1 for a negative control and a plasmid-encoding cPOP2 for a positive control. Because cPOP2-specific transcripts were not found in all cell lines tested, we also verified the quality of the cDNA template by performing the PCR using $\beta$-actin-specific primers, which amplified a 533-bp product. PCR products were excised from agarose gels and confirmed by DNA sequencing to correspond to $\mathrm{cPOP} 2$.

cPOP2 interacts with ASC and PAN1. cPOP1 and vPOPs regulate the activation of $\mathrm{PYD}$-dependent immune effectors by PYD-PYD interaction with the central adaptor protein ASC. cPOP2 further shows highest sequence similarity to the PYD of PAN1, a PYD-NLR protein. The ability of cPOP2 to bind to ASC and PAN1 was therefore investigated by an in vitro glutathione $S$-transferase pull-down assay using ASC and PAN1 as prototypical examples. A recombinant cPOP2 protein was expressed as a GST fusion protein in E. coli BL21 and affinity purified, whereas the PYDs of PAN1 and ASC were generated by in vitro translation. These in vitro interaction studies confirmed that cPOP2 is capable of interacting not only with ASC but also with the PYD of PAN1 (Fig. 3A). No interaction was detectable between ASC and the PYD of PAN1 with the GST control, demonstrating the specificity of these interactions (Fig. 3A).

We next investigated whether the interaction between cPOP2 and ASC or PAN1 can also occur in vivo. The HAtagged PYD of ASC (ASC-PYD) was coexpressed with myctagged cPOP2 (or as a control, cPOP1) in HEK293T cells by transient cotransfection. Coimmunoprecipitation experiments of cleared protein lysates with anti-myc Sepharose demonstrated the presence of HA-tagged ASC-PYD in immune complexes containing myc-tagged cPOP1 or cPOP2 but not in control immune complexes. These findings indicated that cPOP2 can form complexes with ASC in vivo, reminiscent of cPOP1 (Fig. 3B). To test the interaction between CPOP2 and PAN1, we first coexpressed the myc-tagged PYD of PAN1 (PAN1-PYD) and Flag-tagged cPOP2 into HEK293T cells. Coimmunoprecipitation experiments of cleared protein lysates with anti-Flag Sepharose demonstrated the presence of myctagged PAN1-PYD in immune complexes containing Flagtagged cPOP2 but not in control immune complexes (Fig. 3C). Similarly, coexpression of untagged full-length PAN1 with myc-tagged cPOP2 in HEK293T cells followed by coimmunoprecipitation of cleared protein lysates further confirmed specific binding of cPOP2 to PAN1 (Fig. 3D). To test the interaction of cPOP2 and PAN1 also under endogenous conditions, we transiently transfected large and small amounts of myctagged cPOP2 into Jurkat cells, because no antibody specific for cPOP2 is currently available and Jurkat cells express PAN1 (2). Coimmunoprecipitation of myc-containing immune complexes from cleared protein lysates demonstrated the presence of endogenous PAN1 in a dose-dependent manner (Fig. 3E). These experiments demonstrated that $\mathrm{cPOP} 2$ is capable of binding to ASC and PAN1 and that the binding is mediated by PYD-PYD interaction.

POPs can impair PYD-mediated signal transduction by binding to the PYD of ASC but may also block the PYD of PYD-NLR family proteins, such as PAN1, to prevent recruitment of ASC by PYD-PYD interaction. To investigate this possibility, we performed direct yeast two-hybrid interactions between cPOP2 and several PYD-NLR family proteins. cPOP2 was fused to the DNA-binding domain of LexA, whereas the PYDs of PYD-NLR family proteins were fused to the activation domain of B42, because we expect that the interaction will occur between the PYDs of PYD-NLRs and cPOP2, as shown for ASC and PAN1 (Fig. 3F). Results were confirmed by performing a reciprocal experiment. This screen identified several PYD-NLR family proteins as potential binding partners of cPOP2. cPOP2 displays highest sequence similarity to the PYD of PAN1 (Fig. 1B), which consistently conferred the strongest growth of yeast on selective plates and yielded the highest $\beta$-galactosidase activity. Also, the PYDs of PAN2 (PYPAF4, NALP4, CLR19.5), PAN6 (PYPAF7, NALP12, Monarch-1, CLR19.3), NALP1 (NAC, CARD7, DEFCAP, CLR17.1), and ASC, which all show significant similarities to CPOP2, were identified as potential cPOP2-interacting proteins. The PYDs of pyrin, cryopyrin (CIAS1, PYPAF1, NALP3, CLR1.1), PAN5 (NALP10, PYNOD, NOD8, CLR11.1), and PAN10 (PYPAF6, NALP11, NOD17, CLR19.6) did not interact with cPOP2 under these conditions, demonstrating the specificity and selectivity of the identified cPOP2 interactions. 


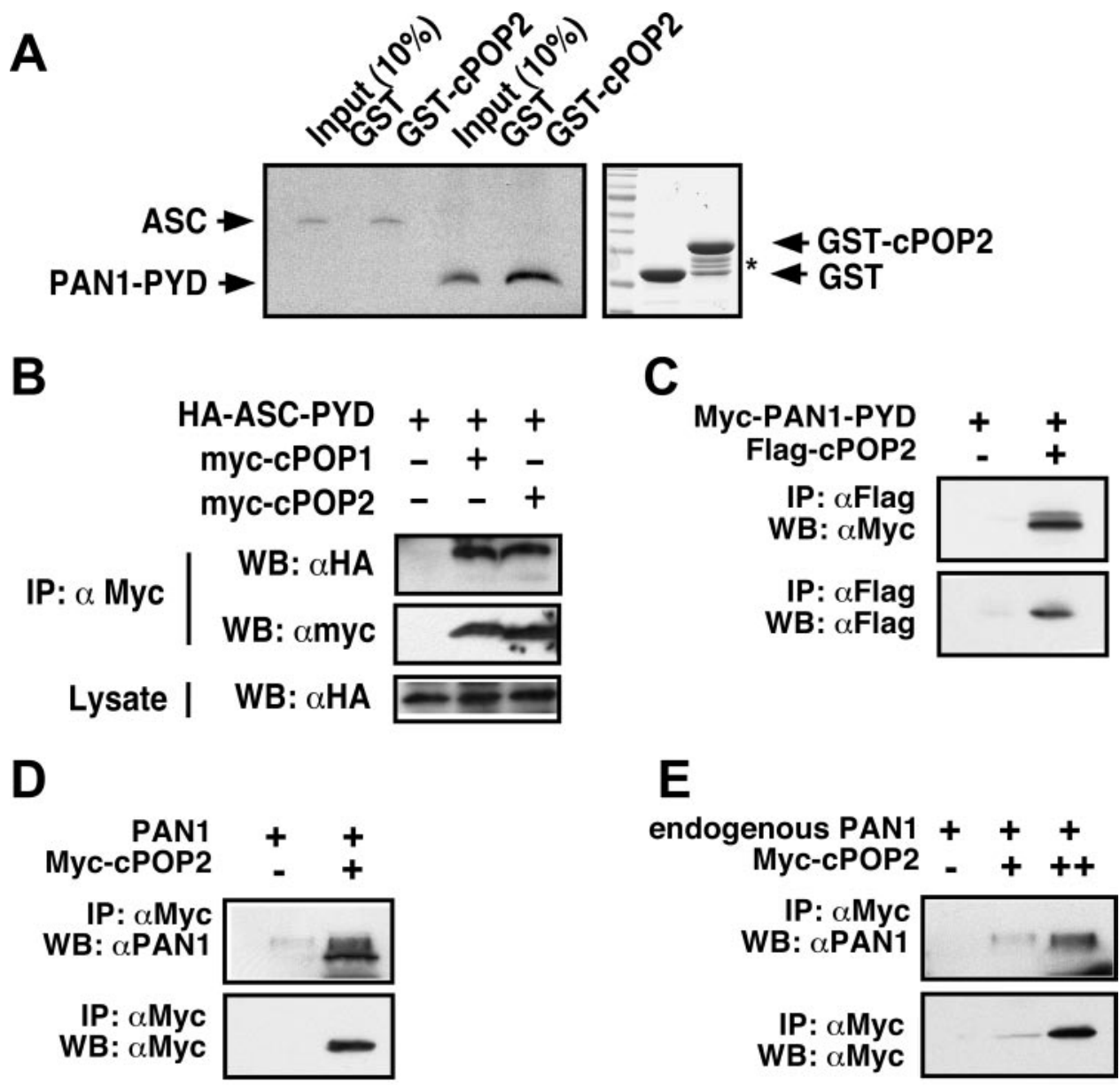

$\mathbf{F}$

\begin{tabular}{|l|c|c|c|c|c|c|c|c|c|}
\hline & ASC & $\begin{array}{c}\text { Cryo- } \\
\text { pyrin }\end{array}$ & Pyrin & PAN1 & PAN2 & PAN5 & PAN6 & PAN10 & NALP1 \\
\hline CPOP2 & + & - & - & ++ & + & - & + & - & ++ \\
\hline
\end{tabular}

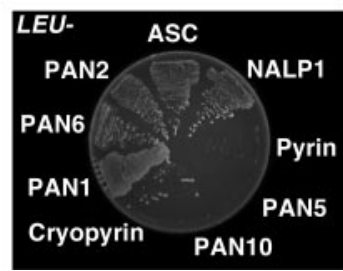

FIG. 3. cPOP2 associates with ASC and PYD-NLR family proteins. (A) In vitro binding between cPOP2, ASC, and PAN1. ASC and the PYD of PAN1 were in vitro translated, labeled with biotin, and subjected to in vitro GST pull-down assays using GST-POP2 and a GST control immobilized on GSH-Sepharose, as indicated. Protein complexes were separated by SDS-PAGE and transferred onto polyvinylidene difluoride (PVDF) membranes, and bound proteins were visualized by immunoblotting with streptavidin-HRP and ECL-Plus (Amersham Pharmacia Biotech) detection. The recombinant GST-cPOP2 and GST proteins are visualized by Coomassie blue staining. The asterisk denotes several degradation products of $\mathrm{cPOP} 2$, which did not affect this assay. (B) In vivo binding between cPOP2 and ASC. HEK293T cells were transiently transfected with the HA-tagged PYD of ASC (ASC-PYD), myc-tagged cPOP1, and myc-tagged cPOP2, as indicated. At $36 \mathrm{~h}$ posttransfection, clarified and normalized cell lysates were subjected to coimmunoprecipitation, using immobilized anti-myc antibodies (Santa Cruz Biotechnology). Immune complexes were separated by SDS-PAGE, transferred onto PVDF membranes, and probed with anti-HA antibodies directly conjugated to HRP and detected with ECL (Amersham Pharmacia Biotech). Membranes were stripped and reprobed with anti-myc-HRP antibodies. Five percent of the total lysate was also immunoblotted with HA-HRP antibodies to control for ASC-PYD expression. IP, immunoprecipitation; WB, Western blot. (C, D, E) In vivo binding between cPOP2 and PAN1. HEK293T cells were transiently transfected with the myc-tagged PYD of PAN1 (PAN1-PYD) and Flag-tagged cPOP2 (C) or full-length, untagged PAN1 and myc-tagged cPOP2 (D), as indicated. At $36 \mathrm{~h}$ posttransfection, clarified and normalized cell lysates were subjected to coimmunoprecipitation, using immobilized anti-Flag antibodies (Sigma) (C) or immobilized anti-myc antibodies (Santa Cruz Biotechnology) (D). Immune complexes were separated by SDS-PAGE, transferred onto PVDF membranes, and probed with anti-myc antibodies directly conjugated to HRP (Santa Cruz Biotechnology) (C) or with anti-PAN1 antibodies (Imgenex) and secondary anti-rabbit antibodies conjugated to HRP (Amersham Pharmacia Biotech) and detected with ECL and ECL-Plus (Amersham Pharmacia Biotech) (D). Membranes were stripped and reprobed with anti-Flag-HRP antibodies (Sigma) (C) or anti-myc HRP antibodies (D). Jurkat cells were transiently transfected with small $(+)$ and large $(++)$ amounts of myc-tagged cPOP2, and cleared lysates were coimmunoprecipitated at $36 \mathrm{~h}$ posttransfection, using immobilized anti-myc antibodies (Santa Cruz Biotechnology) (E). Immune complexes were separated by SDS-PAGE, transferred onto PVDF membranes and probed with anti-PAN1 antibodies (Imgenex) and secondary anti-rabbit HRP antibodies (Amersham Pharmacia), and detected with ECL-Plus (Amersham Pharmacia Biotech). Membranes were stripped and reprobed with anti-myc- 
cPOP2 colocalizes with PAN1 and the central PYD-containing adaptor protein ASC. CPOP1 and vPOPs show a distinct intracellular localization to punctate structures in the cytoplasm and to the nucleus $(7 \mathrm{a}, 13,29)$. When coexpressed with the central adaptor protein ASC, cPOP1 is recruited into ASC-formed specks. In contrast, this redistribution into specks was not observed with vPOPs, which instead colocalized with ASC in smaller, cytoplasmic vesicular structures (7a, 29). Because cPOP2 is capable of binding to ASC and PAN1, we used immunofluorescence to investigate whether $\mathrm{CPOP} 2$ can also colocalize with ASC or PAN1, upon transient coexpression in HEK293 cells. cPOP2 localized primarily to vesicular cytoplasmic structures and the nuclei of cells (Fig. 4A). Coexpression with ASC (Fig. 4C) resulted in partial colocalization of $\mathrm{CPOP} 2$ with ASC to these vesicular structures in the cytoplasm (Fig. 4B).

ASC forms inflammasomes, which require activation of a specific PYD-NLR family member by pathogen-associated molecular patterns. cPOP2 shows highest similarity to the PYD of PAN1, which is one of the PYD-NLRs that recruits ASC to form a specific inflammasome $(1,2)$. Transient expression of PAN1 in HEK293 cells showed a very similar localization to punctate cytoplasmic structures and the nucleus (Fig. 4D). Coexpression with cPOP2 resulted in efficient colocalization of both proteins to these punctate cytoplasmic structures, but neither of the proteins localized any longer to the nucleus (Fig. 4E).

ASC can also form characteristic cytoplasmic structures, referred to as specks, which are large, perinuclear aggregates (24). ASC-interacting proteins, including cPOP1 and vPOPs, are frequently recruited into these structures (7a, 29). cPOP2 was not recruited into ASC-formed specks but was excluded from these structures (Fig. 4F). Also, PAN1 was not recruited into ASC-formed specks under these conditions (data not shown). In contrast, cPOP1 efficiently colocalized with ASC to these specks (Fig. 4G).

cPOP2 interferes with PYD-mediated activation of caspase-1. The best-characterized effector protein downstream of the PYD signal transduction pathway is caspase-1. Procaspase-1 is activated upon recruitment to inflammasomes by CARD-CARD interactions mediated by ASC. Interaction of cPOP1 and vPOPs with ASC impairs PYD-mediated activation of downstream effectors by preventing association with upstream PYD-NLR family proteins $(7 \mathrm{a}, 29)$. Because cPOP2 associates with inflammasome-associated proteins, we investigated the effect of cPOP2 interaction with ASC or PAN1 on PYD-mediated activation of caspase- 1 . The PYD signal transduction pathway was reconstituted in HEK293N cells, which are deficient in endogenous components of this pathway. Coexpression of a constitutively active cryopyrin (R260W) (an
NLR family member), ASC, pro-caspase- 1 , and pro-IL-1 $\beta$ leads to activation of the PYD-mediated signal transduction pathway, which can be measured by secretion of bioactive IL-1 $\beta$. The disease-associated cryopyrin mutant R260W interacts more efficiently with ASC and promotes ASC oligomerization, activation of pro-caspase- 1 , and subsequently IL-1 $\beta$ secretion, even in the absence of a ligand (8). Coexpression of cPOP2 with ASC and cryopyrin (R260W) impaired ASC-mediated activation of caspase- 1 in a dose-dependent manner, as measured by secretion of bioactive IL-1 $\beta$, which is processed from pro-IL-1 $\beta$ by caspase-1 (Fig. 5A). As previously reported, coexpression of ASC and PAN1 also induces activation of pro-caspase-1 $(1,2)$. Coexpression of cPOP2 with ASC and PAN1 also impaired activation of caspase-1 by the PAN1containing inflammasome in a dose-dependent manner (Fig. $5 \mathrm{~B})$. These results demonstrate a potential role for cPOP2 in modulating activation of caspase- 1 by specific inflammasomes.

\section{DISCUSSION}

PYD-containing proteins have emerged as important mediators of inflammatory responses during host defense. The PYD signal transduction pathway is triggered by the recognition of specific pathogen-associated molecular patterns by PYD-containing pathogen recognition receptors (PYD-NLR family proteins). Active PYD-NLRs recruit the adaptor protein ASC to form specific inflammasomes, where activation of pro-caspase- 1 occurs $(1,22)$. To date, 16 human PYD-NLR family members that form inflammasomes, including NALP1, cryopyrin, and PAN1, have been recognized (31). However, the exact molecular mechanism is currently poorly understood. Specific ligands have thus far been identified only for cryopyrin and NALP1 $(1,14,15,21-23,32)$. Activation of PYD-NLR family proteins results in oligomerization and recruitment of the adaptor protein ASC via PYD-PYD interactions (22). Subsequently, ASC links pathogen recognition to downstream effector activation, including caspase-1-mediated processing and secretion of IL-1 $\beta$, IL-18, and possibly IL-33 (20, 32, 35). Due to the key role of the proinflammatory cytokine IL-1 $\beta$, its generation and receptor binding are regulated at several key areas in mammalian cells, including CARD-only proteins (COPs) and IL-1RA $(6,7)$.

Our identification of cPOP2 in the present study is the first indication of an endogenous inhibitor that controls generation of IL-1 $\beta$ at the initiating step, directly at the level of inflammasome formation. Many of the PYD-containing proteins are inducibly expressed in response to proinflammatory stimuli, which is consistent with their proinflammatory function. Also,

HRP antibodies (Santa Cruz Biotechnology). (F) Direct interaction screen in yeast between cPOP2, ASC, and PYD-NLR family proteins. Yeast two-hybrid assays were performed, scoring for activation of reporter genes encoding leucine (LEU2) and $\beta$-galactosidase (left panel). Plasmid combinations that resulted in growth on leucine-deficient media within 4 days were scored as positive (right panel). The $\beta$-galactosidase activity of each colony was also tested by filter assay and scored according to the time required to yield a blue color. cPOP2 was fused to the DNA binding domain of LexA, and the PYD family proteins were fused to the activation domain of B42. The scoring was obtained by considering also the reciprocal experiments, where cPOP2 was fused to the activation domain of B4, and the PYD family proteins were fused to the DNA binding domain of LexA. In all cases, there was good agreement between data obtained by both experiments and also between the leucine and the $\beta$-galactosidase assays. Note that all plasmids contained only the PYD. Results for PAN2 (PYPAF4, NALP4, CLR19.5), PAN5 (NALP10, PYNOD, NOD8, CLR11.1), PAN6 (PYPAF7, NALP12, Monarch-1, CLR19.3), PAN10 (PYPAF6, NALP11, NOD17, CLR19.6), NAC (CARD7, DEFCAP, NALP1, CLR17.1), and cryopyrin (CIAS1, PYPAF1, NALP3, CLR1.1) are shown. 


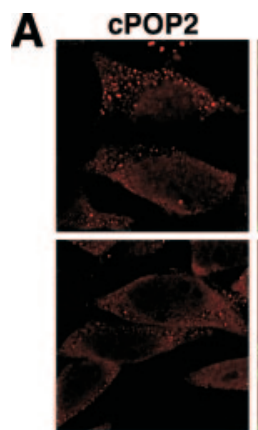

B
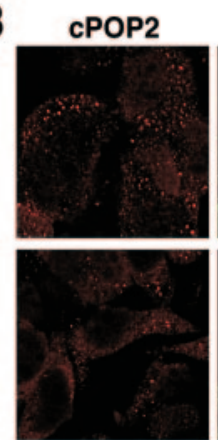

C

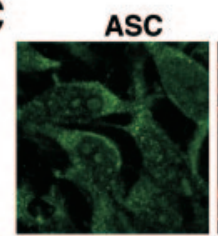

G

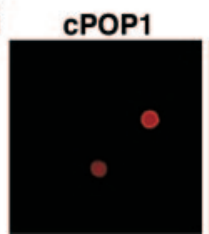

actin

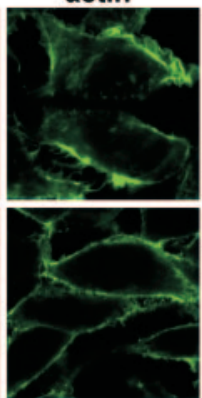

ASC
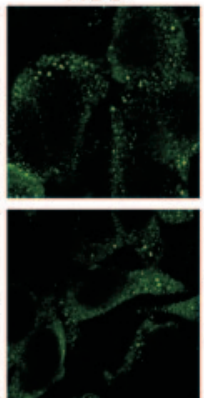

actin

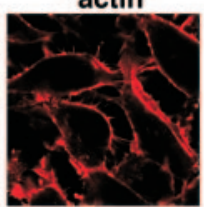

ASC

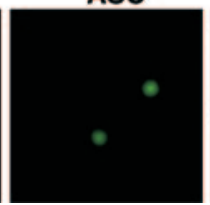

nucleus

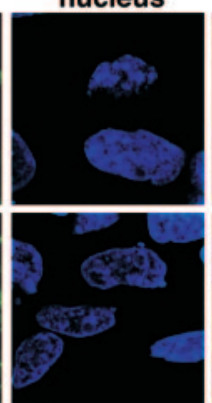

nucleus
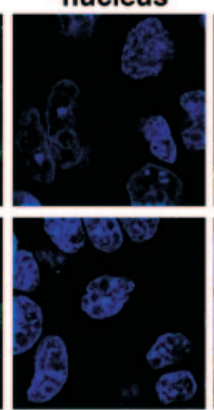

nucleus

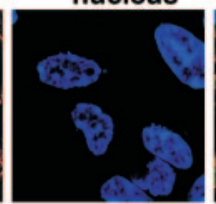

nucleus

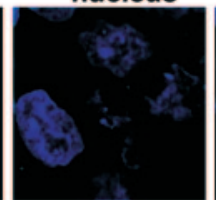

merged

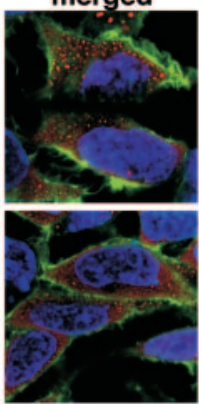

merged
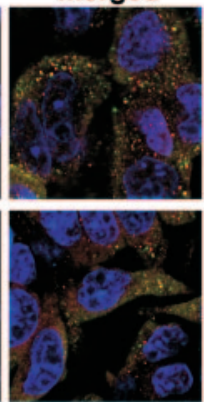

merged

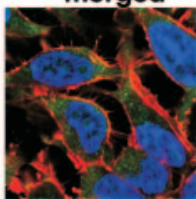

merged

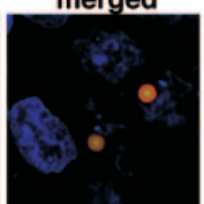

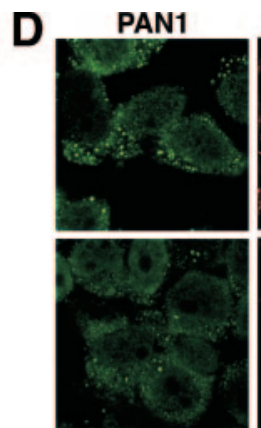
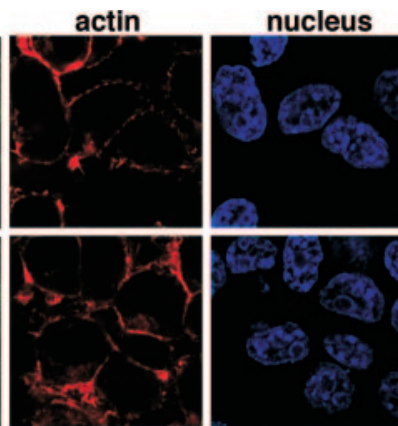

E
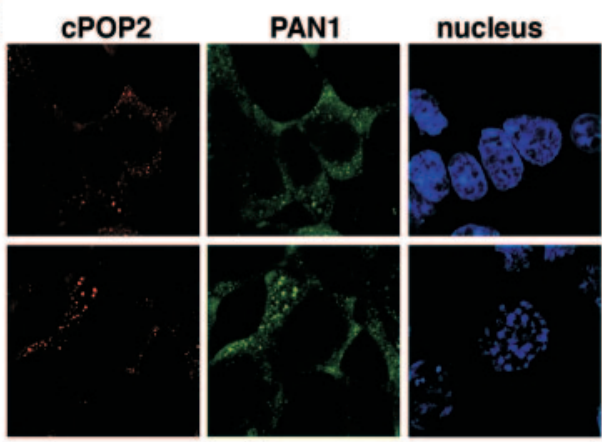

F
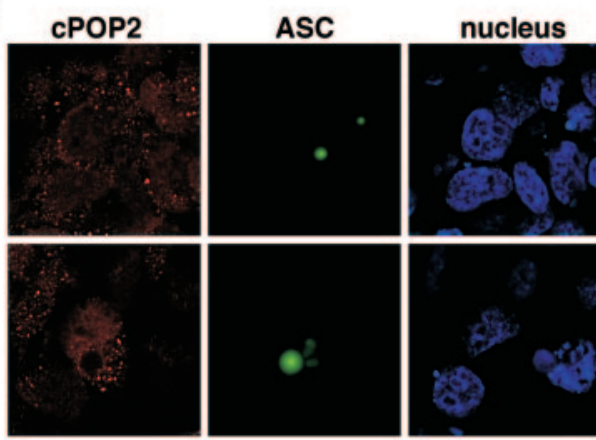

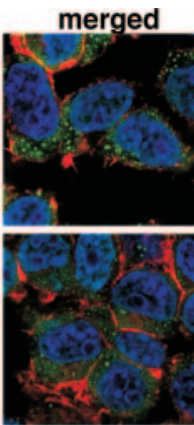

merged
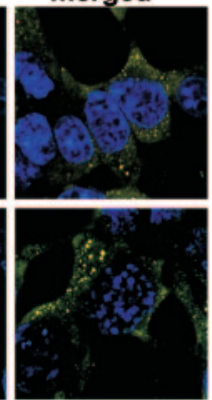

FIG. 4. $\mathrm{cPOP} 2$ colocalizes with ASC and PAN1. Localization of epitope-tagged proteins was analyzed following transient transfection into HEK293T cells. (A) Myc-tagged cPOP2 was immunostained with a rabbit polyclonal anti-myc antibody and visualized with an Alexa Fluor 546-conjugated anti-rabbit antibody. Actin was visualized with Alexa Fluor 488-conjugated phalloidin, and the nucleus was stained with ToPro-3. Shown from left to right are myc-tagged cPOP2 (red), actin (green), the nucleus (blue), and a merged image. (B) Myc-tagged cPOP2 and Flag-tagged ASC and were immunostained with rabbit polyclonal anti-myc and mouse monoclonal anti-Flag antibodies and visualized with Alexa Fluor 546- and 488-conjugated anti-rabbit and anti-mouse antibodies, respectively. The nucleus was stained with ToPro-3. Shown from left to right are myc-tagged cPOP2 (red), Flag-tagged ASC (green), the nucleus (blue), and a merged image. (C) Flag-tagged ASC was immunostained with a mouse monoclonal anti-Flag antibody and visualized with Alexa Fluor 488-conjugated anti-mouse antibodies. The nucleus was stained with ToPro-3. Shown from left to right are Flag-tagged ASC (green), actin (red), the nucleus (blue), and a merged image. (D) HA-tagged PAN1 was immunostained with a rat polyclonal anti-HA antibody and visualized with Alexa Fluor 488-conjugated anti-rat antibodies. The nucleus was stained with ToPro-3. Shown from left to right are HA-tagged PAN1 (green), actin (red), the nucleus (blue), and a merged image. (E) Myc-tagged cPOP2 and HA-tagged PAN1 were immunostained with rabbit polyclonal anti-myc and rat polyclonal anti-HA antibodies and visualized with Alexa Fluor 546- and 488-conjugated anti-rabbit and anti-rat antibodies, respectively. The nucleus was stained with ToPro-3. Shown from left to right are myc-tagged cPOP2 (red), HA-tagged PAN1 (green), the nucleus (blue), and a merged image. (F) Myc-tagged cPOP2 and Flag-tagged ASC were immunostained as described for panel B, and a representative cell that showed an ASC-formed speck was imaged. Shown from left to right are myc-tagged cPOP2 (red), Flag-tagged ASC (green), the nucleus (blue), and a merged image. (G) Myc-tagged cPOP1 and Flag-tagged ASC were immunostained as described above, and a representative cell that showed an ASC-formed speck was imaged. Shown from left to right are myc-tagged cPOP1 (red), Flag-tagged ASC (green), the nucleus (blue), and a merged image.

cPOP2 was induced at the mRNA level in THP-1 monocytes in response to lipopolysaccharide (LPS) stimulation, emphasizing its role during inflammation. cPOP2 shows high similarity to several PYD-NLR family proteins and ASC. The highest similarity is found to the PYD of PAN1, and cPOP2 is capable of interacting with PAN1. Unlike cPOP1, which is clustered with other PYD-containing proteins, including pyrin and ASC, on chromosome 16, cPOP2 is located on chromosome $3 \mathrm{q} 28$ alone. cPOP2 displays $40 \%$ similarity with cPOP1. The observation that cPOP2 also interacts with the PYD of PYD-NLR family proteins provides additional insights into inflammasome formation and provides evidence that POPs interact not only with 


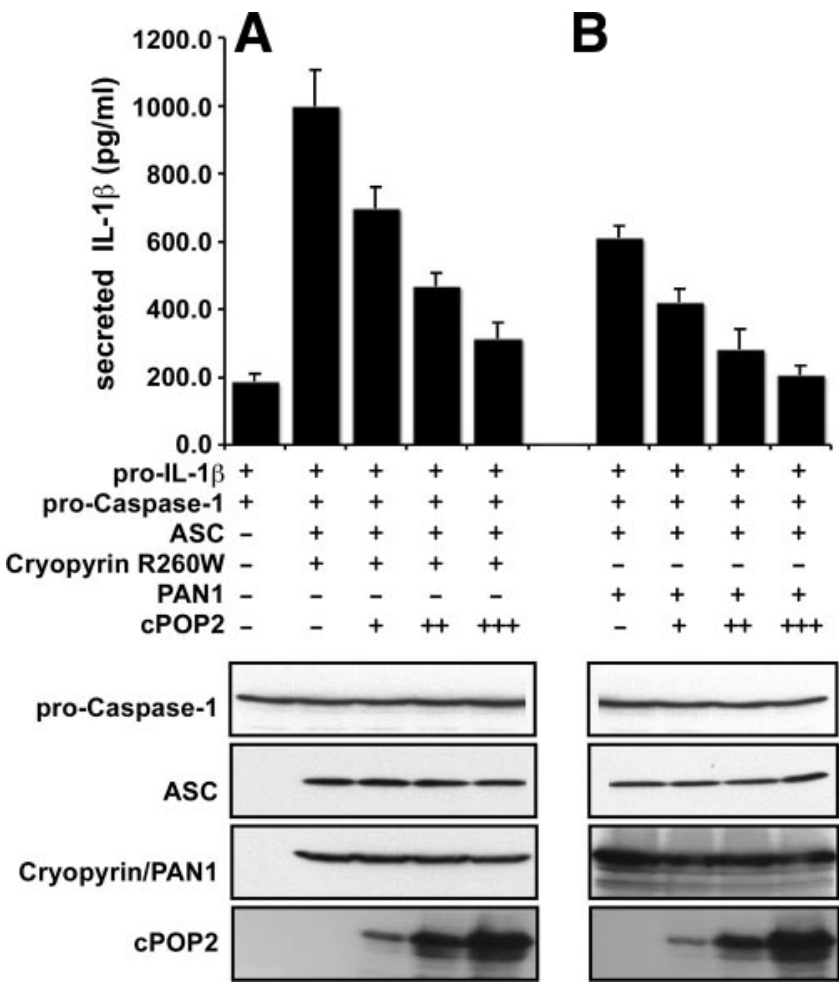

FIG. 5. cPOP2 inhibits caspase-1-mediated processing of pro-IL$1 \beta$. HEK293N cells were transiently transfected in triplicate with expression constructs for pro-caspase-1, murine pro-IL-1 $\beta$, ASC, cPOP2, and cryopyrin (R260W) (A) or PAN1 (B) (small dose, +; medium dose, ++ ; and large dose, +++ ), as indicated. At $36 \mathrm{~h}$ posttransfection, secreted IL-1 $\beta$ was measured by ELISA (BD Pharmingen) from normalized culture supernatants using a standard curve generated with recombinant IL-1 $\beta$. Data are presented as picograms per milliliter of secreted IL-1 $\beta$ (mean \pm standard deviation; $n=3$ ). Transfected cells were also directly lysed in Laemmli buffer and analyzed by SDS-PAGE immunoblotting for the expression of all constructs, except pro-IL-1 $\beta$, which was measured by ELISA.

the adaptor ASC but also directly with PYD-NLR family proteins to regulate activation of pro-caspase-1.

cPOP2 localizes to cytoplasmic vesicular structures but is also found diffusely throughout the nucleus, similar to cPOP1 (29). ASC localizes to punctate structures in the cytoplasm, or it forms characteristic cytoplasmic aggregates, referred to as specks, to which ASC-interacting proteins, including cPOP1, are frequently recruited. Some colocalization between cPOP2 and ASC can be observed in these vesicular structures in the cytoplasm, but cPOP2 is not recruited to ASC-containing specks, and specks are rarely formed when cPOP2 is coexpressed with ASC. One explanation might be that cPOP2 impairs the aggregation of ASC with other proteins that are essential for speck formation, such as PYD-NLR family proteins. These differences further suggest a distinct functional mechanism of cPOP1 compared to that of cPOP2. Expression of PAN1 resulted in localization similar to that observed for cPOP2, and both proteins efficiently colocalize when coexpressed. Like cPOP1 and vPOPs, cPOP2 also interacts with ASC, the central adaptor protein of the PYD signal transduction pathway. We identified several PYD-NLR family members as potential binding partners for cPOP2, verified binding to PAN1, and showed by coimmunoprecipitation and GST pull-down experiments that the binding is mediated by PYDPYD interaction. We also expect that, based on yeast twohybrid assay results, PAN2, PAN6, and NALP1 are capable of binding to cPOP2. Because we did not test all known PYDcontaining proteins, there might be more potential PYD-containing binding partners of cPOP2 (Table 1).

Binding of ASC to activated PYD-NLR family proteins results in the formation of inflammasomes, which are required for activation of pro-caspase- $1(1,22)$. Activation of pro-caspase- 1 is required for the processing, activation, and subsequent secretion of the proinflammatory cytokines pro-IL-1 $\beta$, pro-IL-18, and pro-IL-33 $(10,26)$. cPOP2 impaired cryopyrin- and ASCmediated activation of pro-caspase-1, possibly explained by the impaired formation of a cryopyrin-containing inflammasome in the presence of cPOP2. cPOP2 also impaired activation of pro-caspase-1 from a PAN1-containing inflammasome. Assembly of the cryopyrin-containing inflammasome is probably blocked by binding to the PYD of ASC, whereas the PAN1containing inflammasome could be inhibited by simultaneously blocking the PYDs of ASC and PAN1, which would impair the recruitment of ASC to PAN1. The lower secretion of IL-1 $\beta$ from PAN1-expressing cells than from cryopyrin (R260W)expressing cells might be due to the higher activity of the R260W-mutated cryopyrin protein, which is found in patients with autoinflammatory disorders, as opposed to the wild-type PAN1 protein (8). The sequence similarity between cPOP2 and cPOP1 is $40 \%$ and is 31 to $36 \%$ between cPOP2 and vPOPs. Determination of whether cPOP2 and other POPs function to "fine-tune" inflammasome assembly, prevent formation of specific inflammasomes, or participate in the termi-

TABLE 1. Similarity of cPOP2 to known human PYD-containing proteins ${ }^{a}$

\begin{tabular}{|c|c|}
\hline Protein & $\begin{array}{c}\text { cPOP2 } \\
\text { (\% sequence similarity) }\end{array}$ \\
\hline PAN1..... & .................69 \\
\hline PAN7..... & .................50 \\
\hline PAN3..... & ..................43 \\
\hline PAN6..... & ….............42 \\
\hline PAN2..... & …............41 \\
\hline PAN12.. & …............40 \\
\hline cPOP1 ... & ….............. 40 \\
\hline PAN8..... & $\ldots . . . \ldots \ldots \ldots \ldots . . . .38$ \\
\hline AIM $2 \ldots . .$. & ….................. \\
\hline ASC ...... & 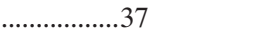 \\
\hline Pyrin ....... & 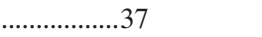 \\
\hline PAN5..... & 37 \\
\hline IFIX........ & $\ldots \ldots \ldots \ldots \ldots . . .35$ \\
\hline PAN9..... & $\ldots . . . \ldots \ldots \ldots . . .34$ \\
\hline PAN13... & 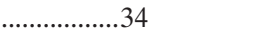 \\
\hline Cryopyri & $\ldots 33$ \\
\hline NALP1.. & .....32 \\
\hline PAN10... & $\ldots 32$ \\
\hline PAN11... &. .30 \\
\hline IFI16....... & $\ldots 30$ \\
\hline PAN14... & $\ldots .28$ \\
\hline MNDA.. & $\ldots 27$ \\
\hline PAN4..... & $\ldots .25$ \\
\hline
\end{tabular}

${ }^{a}$ Separate Clustal $\mathrm{W}$ alignments of cPOP2 with the PYD of human PYD containing proteins were performed to calculate percent sequence similarity (identical and conserved amino acid residues). 
nation of caspase-1 activation to prevent systemic inflammation will require further investigation.

In summary, our data provide evidence for the existence of a second cellular POP, which binds to the PYD of ASC and PAN1 and potentially to several other PYD-NLR proteins, to impair inflammasome-mediated activation of pro-caspase- 1 and subsequent processing and secretion of the proinflammatory cytokine IL-1 $\beta$. The existence of two different cellular POPs might provide for more versatile regulation of inflammasome assembly and might allow specific PYD-NLR family proteins to be suppressed and others to be spared.

\section{ACKNOWLEDGMENTS}

This work was supported by Public Health Service grants 5P20RR016440 (to C.S. and D.C.F.) from the National Center for Research Resources (NCRR), a component of the National Institutes of Health (NIH); 1R21-AI067680, 1R03-AI067806 (to C.S.), and R01-AI56324 (to J.C.R.) from the National Institute of Allergy and Infectious Diseases; and 1R01-GM071723 (to C.S.) from the National Institute of General Medical Sciences. We are also grateful for the support from the Alexander Bland Osborn Cancer Center Endowment (to C.S.).

This article's contents are solely the responsibility of the authors and do not necessarily represent the official views of NCRR or NIH.

\section{REFERENCES}

1. Agostini, L., F. Martinon, K. Burns, M. F. McDermott, P. N. Hawkins, and J. Tschopp. 2004. NALP3 forms an IL-1beta-processing inflammasome with increased activity in Muckle-Wells autoinflammatory disorder. Immunity 20:319-325.

2. Bruey, J. M., N. Bruey-Sedano, R. Newman, S. Chandler, C. Stehlik, and J. C. Reed. 2004. PAN1/NALP2/PYPAF2, an inducible inflammatory mediator that regulates NF-kappaB and caspase-1 activation in macrophages. J. Biol. Chem. 279:51897-51907.

3. Brydges, S., and D. L. Kastner. 2006. The systemic autoinflammatory diseases: inborn errors of the innate immune system. Curr. Top. Microbiol. Immunol. 305:127-160.

4. Condorelli, G., G. Vigliotta, A. Cafieri, A. Trencia, P. Andalo, F. Oriente, C. Miele, M. Caruso, P. Formisano, and F. Beguinot. 1999. PED/PEA-15: an anti-apoptotic molecule that regulates FAS/TNFR1-induced apoptosis. Oncogene 18:4409-4415.

5. Conway, K. E., B. B. McConnell, C. E. Bowring, C. D. Donald, S. T. Warren, and P. M. Vertino. 2000. TMS1, a novel proapoptotic caspase recruitment domain protein, is a target of methylation-induced gene silencing in human breast cancers. Cancer Res. 60:6236-6242.

6. Dinarello, C. A. 2005. Blocking IL-1 in systemic inflammation. J. Exp. Med. 201:1355-1359.

7. Dinarello, C. A. 1998 . Interleukin-1 beta, interleukin-18, and the interleukin-1 beta converting enzyme. Ann. N. Y. Acad. Sci. 856:1-11.

7a.Dorfleutner, A., S. J. Talbott, N. B. Bryan, K. N. Funya, J. C. Reed, X. Shi, D. C. Flynn, Y. Rojanasakul, and C. Stehlik. Poxvirus PYRIN-only proteins are modulators of the host immune response.Virus Genes, in press.

8. Dowds, T. A., J. Masumoto, L. Zhu, N. Inohara, and G. Nunez. 2004 Cryopyrin-induced interleukin 1beta secretion in monocytic cells: enhanced activity of disease-associated mutants and requirement for ASC. J. Biol. Chem. 279:21924-21928.

9. Druilhe, A., S. M. Srinivasula, M. Razmara, M. Ahmad, and E. S. Alnemri. 2001. Regulation of IL-1beta generation by Pseudo-ICE and ICEBERG, two dominant negative caspase recruitment domain proteins. Cell Death Differ. 8:649-657.

10. Fantuzzi, G., and C. A. Dinarello. 1999. Interleukin-18 and interleukin-1 beta: two cytokine substrates for ICE (caspase-1). J. Clin. Immunol. 19:1-11.

11. Humke, E. W., S. K. Shriver, M. A. Starovasnik, W. J. Fairbrother, and V. M. Dixit. 2000. ICEBERG: a novel inhibitor of interleukin-1beta generation. Cell 103:99-111.

12. Inohara, N., and G. Nunez. 2003. NODS: intracellular proteins involved in inflammation and apoptosis. Nat. Rev. Immunol. 3:371-381.

13. Johnston, J. B., J. W. Barrett, S. H. Nazarian, M. Goodwin, D. Ricuttio, G. Wang, and G. McFadden. 2005. A poxvirus-encoded pyrin domain protein interacts with ASC-1 to inhibit host inflammatory and apoptotic responses to infection. Immunity 23:587-598.

14. Kanneganti, T. D., M. Body-Malapel, A. Amer, J. H. Park, J. Whitfield, L.
Franchi, Z. F. Taraporewala, D. Miller, J. T. Patton, N. Inohara, and G. Nunez. 2006. Critical role for cryopyrin/Nalp3 in activation of caspase-1 in response to viral infection and double-stranded RNA. J. Biol. Chem. 48: 36560-36568.

15. Kanneganti, T. D., N. Ozoren, M. Body-Malapel, A. Amer, J. H. Park, L. Franchi, J. Whitfield, W. Barchet, M. Colonna, P. Vandenabeele, J. Bertin, A. Coyle, E. P. Grant, S. Akira, and G. Nunez. 2006. Bacterial RNA and small antiviral compounds activate caspase-1 through cryopyrin/Nalp3. Nature 440:232-236.

16. Krueger, A., I. Schmitz, S. Baumann, P. H. Krammer, and S. Kirchhoff. 2001. Cellular FLICE-inhibitory protein splice variants inhibit different steps of caspase- 8 activation at the CD95 death-inducing signaling complex. J. Biol. Chem. 276:20633-20640.

17. Lamkanfi, M., G. Denecker, M. Kalai, K. D'Hondt, A. Meeus, W. Declercq, X. Saelens, and P. Vandenabeele. 2004. INCA, a novel human caspase recruitment domain protein that inhibits interleukin-1beta generation. J. Biol. Chem. 279:51729-51738

18. Lee, S.-H., C. Stehlik, and J. C. Reed. 2001. COP, a CARD-containing protein and inhibitor of pro-interleukin-1b processing. J. Biol. Chem. 276: 34495-34500.

19. Liepinsh, E., R. Barbals, E. Dahl, A. Sharipo, E. Staub, and G. Otting. 2003. The death-domain fold of the ASC PYRIN domain, presenting a basis for PYRIN/PYRIN recognition. J. Mol. Biol. 332:1155-1163.

20. Mariathasan, S., K. Newton, D. M. Monack, D. Vucic, D. M. French, W. P. Lee, M. Roose-Girma, S. Erickson, and V. M. Dixit. 2004. Differential activation of the inflammasome by caspase-1 adaptors ASC and Ipaf. Nature 430:213-218.

21. Mariathasan, S., D. S. Weiss, K. Newton, J. McBride, K. O'Rourke, M. Roose-Girma, W. P. Lee, Y. Weinrauch, D. M. Monack, and V. M. Dixit. 2006. Cryopyrin activates the inflammasome in response to toxins and ATP. Nature 440:228-232.

22. Martinon, F., K. Burns, and J. Tschopp. 2002. The inflammasome: a molecular platform triggering activation of inflammatory caspases and processing of proIL-beta. Mol. Cell 10:417-426.

23. Martinon, F., V. Petrilli, A. Mayor, A. Tardivel, and J. Tschopp. 2006. Gout-associated uric acid crystals activate the NALP3 inflammasome. Nature 440:237-241.

24. Masumoto, J., S. Taniguchi, K. Ayukawa, H. Sarvotham, T. Kishino, N. Niikawa, E. Hidaka, T. Katsuyama, T. Higuchi, and J. Sagara. 1999. ASC, a novel 22-kDa protein, aggregates during apoptosis of human promyelocytic leukemia HL-60 cells. J. Biol. Chem. 274:33835-33838.

25. Moss, B., and J. L. Shisler. 2001. Immunology 101 at poxvirus U: immune evasion genes. Semin. Immunol. 13:59-66.

26. Schmitz, J., A. Owyang, E. Oldham, Y. Song, E. Murphy, T. K. McClanahan, G. Zurawski, M. Moshrefi, J. Qin, X. Li, D. M. Gorman, J. F. Bazan, and R. A. Kastelein. 2005. IL-33, an interleukin-1-like cytokine that signals via the IL-1 receptor-related protein ST2 and induces T helper type 2-associated cytokines. Immunity 23:479-490.

27. Seet, B. T., J. B. Johnston, C. R. Brunetti, J. W. Barrett, H. Everett, C. Cameron, J. Sypula, S. H. Nazarian, A. Lucas, and G. McFadden. 2003. Poxviruses and immune evasion. Annu. Rev. Immunol. 21:377-423.

28. Stehlik, C., L. Fiorentino, A. Dorfleutner, J. M. Bruey, E. M. Ariza, J. Sagara, and J. C. Reed. 2002. The PAAD/PYRIN-family protein ASC is a dual regulator of a conserved step in nuclear factor kappaB activation pathways. J. Exp. Med. 196:1605-1615.

29. Stehlik, C., M. Krajewska, K. Welsh, S. Krajewski, A. Godzik, and J. C. Reed. 2003. The PAAD/PYRIN-only protein POP1/ASC2 is a modulator of ASC-mediated nuclear-factor-kappaB and pro-caspase-1 regulation. Biochem. J. 373:101-113.

30. Stehlik, C., S. H. Lee, A. Dorfleutner, A. Stassinopoulos, J. Sagara, and J. C. Reed. 2003. Apoptosis-associated speck-like protein containing a caspase recruitment domain is a regulator of procaspase-1 activation. J. Immunol. 171:6154-6163.

31. Stehlik, C., and J. C. Reed. 2004. The PYRIN connection: novel players in innate immunity and inflammation. J. Exp. Med. 200:551-558.

32. Sutterwala, F. S., Y. Ogura, M. Szczepanik, M. Lara-Tejero, G. S. Lichtenberger, E. P. Grant, J. Bertin, A. J. Coyle, J. E. Galan, P. W. Askenase, and R. A. Flavell. 2006. Critical role for NALP3/CIAS1/Cryopyrin in innate and adaptive immunity through its regulation of caspase-1. Immunity 24:317-327.

33. Ting, J. P., and B. K. Davis. 2005. CATERPILLER: a novel gene family important in immunity, cell death, and diseases. Annu. Rev. Immunol. 23: 387-414.

34. Tschopp, J., F. Martinon, and K. Burns. 2003. NALPs: a novel protein family involved in inflammation. Nat. Rev. Mol. Cell Biol. 4:95-104.

35. Yamamoto, M., K. Yaginuma, H. Tsutsui, J. Sagara, X. Guan, E. Seki, K. Yasuda, M. Yamamoto, S. Akira, K. Nakanishi, T. Noda, and S. Taniguchi. 2004. ASC is essential for LPS-induced activation of procaspase-1 independently of TLR-associated signal adaptor molecules. Genes Cells 9:1055-1067. 